Cochrane Database of Systematic Reviews

\title{
Supportive interventions for enhancing dietary intake in malnourished or nutritionally at-risk adults (Review)
}

Baldwin C, Kimber KL, Gibbs M, Weekes CE

Baldwin C, Kimber KL, Gibbs M, Weekes CE.

Supportive interventions for enhancing dietary intake in malnourished or nutritionally at-risk adults.

Cochrane Database of Systematic Reviews 2016, Issue 12. Art. No.: CD009840.

DOI: 10.1002/14651858.CD009840.pub2.

www.cochranelibrary.com 
TABLE OF CONTENTS

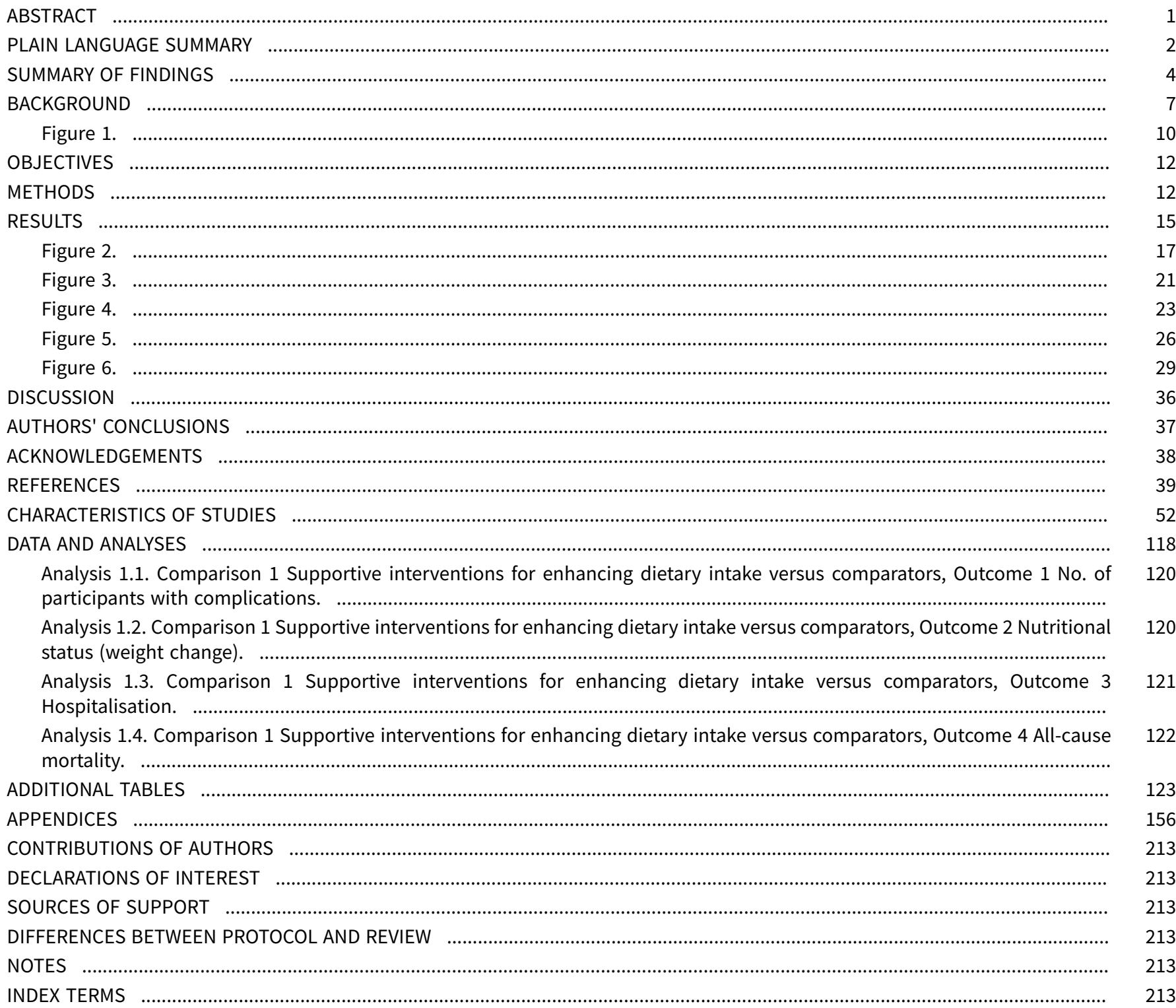


[Intervention Review]

\section{Supportive interventions for enhancing dietary intake in malnourished or nutritionally at-risk adults}

Christine Baldwin ${ }^{1}$, Katherine L Kimber², Michelle Gibbs ${ }^{1}$, Christine Elizabeth Weekes ${ }^{3}$

1Diabetes \& Nutritional Sciences Division, School of Medicine, King's College London, London, UK. 2Diabetes \& Nutritional Sciences Division, School of Medicine, King's College London, London, UK. 3Department of Nutrition \& Dietetics, Guy's \& St Thomas NHS Foundation Trust, London, UK

Contact: Christine Baldwin, Diabetes \& Nutritional Sciences Division, School of Medicine, King's College London, Franklin Wilkins Building, 150 Stamford Street, London, SE1 9NH, UK. christine.baldwin@kcl.ac.uk.

Editorial group: Cochrane Metabolic and Endocrine Disorders Group.

Publication status and date: New, published in Issue 12, 2016.

Citation: Baldwin C, Kimber KL, Gibbs M, Weekes CE. Supportive interventions for enhancing dietary intake in malnourished or nutritionally at-risk adults. Cochrane Database of Systematic Reviews 2016, Issue 12. Art. No.: CD009840. DOI: 10.1002/14651858.CD009840.pub2.

Copyright ( 2016 The Cochrane Collaboration. Published by John Wiley \& Sons, Ltd.

\section{A B S T R A C T}

\section{Background}

Supportive interventions such as serving meals in a dining room environment or the use of assistants to feed patients are frequently recommended for the management of nutritionally vulnerable groups. Such interventions are included in many policy and guideline documents and have implications for staff time but may incur additional costs, yet there appears to be a lack of evidence for their efficacy.

\section{Objectives}

To assess the effects of supportive interventions for enhancing dietary intake in malnourished or nutritionally at-risk adults.

\section{Search methods}

We identified publications from comprehensive searches of the Cochrane Library, MEDLINE, Embase, AMED, British Nursing Index, CINAHL, SCOPUS, ISI Web of Science databases, scrutiny of the reference lists of included trials and related systematic reviews and handsearching the abstracts of relevant meetings. The date of the last search for all databases was 31 March 2013. Additional searches of CENTRAL, MEDLINE, ClinicalTrials.gov and WHO ICTRP were undertaken to September 2016. The date of the last search for these databases was 14 September 2016.

\section{Selection criteria}

Randomised controlled trials of supportive interventions given with the aim of enhancing dietary intake in nutritionally vulnerable adults compared with usual care.

\section{Data collection and analysis}

Three review authors and for the final search, the editor, selected trials from titles and abstracts and independently assessed eligibility of selected trials. Two review authors independently extracted data and assessed risk of bias, as well as evaluating overall quality of the evidence utilising the GRADE instrument, and then agreed as they entered data into the review. The likelihood of clinical heterogeneity amongst trials was judged to be high as trials were in populations with widely different clinical backgrounds, conducted in different healthcare settings and despite some grouping of similar interventions, involved interventions that varied considerably. We were only able, therefore, to conduct meta-analyses for the outcome measures, 'all-cause mortality', 'hospitalisation' and 'nutritional status (weight change)'. 


\section{Main results}

Forty-one trials (10,681 participants) met the inclusion criteria. Trials were grouped according to similar interventions (changes to organisation of nutritional care ( $N=13 ; 3456$ participants), changes to the feeding environment $(\mathrm{N}=5 ; 351$ participants $)$, modification of meal profile or pattern $(\mathrm{N}=12 ; 649$ participants), additional supplementation of meals $(\mathrm{N}=10 ; 6022$ participants) and home meal delivery systems ( $N=1 ; 203$ participants). Follow-up ranged from 'duration of hospital stay' to 12 months.

The overall quality of evidence was moderate to very low, with the majority of trials judged to be at an unclear risk of bias in several risk of bias domains. The risk ratio (RR) for all-cause mortality was 0.78 ( $95 \%$ confidence interval $(\mathrm{Cl}) 0.66$ to 0.92$) ; \mathrm{P}=0.004 ; 12$ trials; 6683 participants; moderate-quality evidence. This translates into $26(95 \% \mathrm{Cl} 9$ to 41$)$ fewer cases of death per 1000 participants in favour of supportive interventions. The RR for number of participants with any medical complication ranged from 1.42 in favour of control compared with 0.59 in favour of supportive interventions (very low-quality evidence). Only five trials (4451 participants) investigated health-related quality of life showing no substantial differences between intervention and comparator groups. Information on patient satisfaction was unreliable. The effects of supportive interventions versus comparators on hospitalisation showed a mean difference (MD) of -0.5 days $(95 \% \mathrm{Cl}-2.6$ to 1.6$) ; \mathrm{P}=0.65 ; 5$ trials; 667 participants; very low-quality evidence. Only three of 41 included trials (4108 participants; very low-quality evidence) reported on adverse events, describing intolerance to the supplement (diarrhoea, vomiting; 5/34 participants) and discontinuation of oral nutritional supplements because of refusal or dislike of taste (567/2017 participants). Meta-analysis across 17 trials with adequate data on weight change revealed an overall improvement in weight in favour of supportive interventions versus control: MD $0.6 \mathrm{~kg}$ (95\% Cl 0.21 to 1.02); 2024 participants; moderate-quality evidence. A total of 27 trials investigated nutritional intake with a majority of trials not finding marked differences in energy intake between intervention and comparator groups. Only three trials (1152 participants) reported some data on economic costs but did not use accepted health economic methods (very low-quality evidence).

\section{Authors' conclusions}

There is evidence of moderate to very low quality to suggest that supportive interventions to improve nutritional care results in minimal weight gain. Most of the evidence for the lower risk of all-cause mortality for supportive interventions comes from hospital-based trials and more research is needed to confirm this effect. There is very low-quality evidence regarding adverse effects; therefore whilst some of these interventions are advocated at a national level clinicians should recognise the lack of clear evidence to support their role. This review highlights the importance of assessing patient-important outcomes in future research.

\section{PLAIN LANGUAGE SUMMARY}

\section{Supportive interventions for improving dietary intake in nutritionally vulnerable groups}

\section{Review question}

Are supportive interventions for improving dietary intake in nutritionally vulnerable groups (malnourished or nutritionally at-risk individuals) effective?

\section{Background}

Serving meals in a dining room, or the use of assistance to help feed people in need and other similar methods are often recommended to help especially sick and elderly people who have lost or are likely to lose weight (nutritionally vulnerable groups). Such supportive interventions are implemented in the health care in many countries but their effects are not well investigated.

\section{Study characteristics}

We included 41 randomised controlled studies (clinical studies where people are randomly put into one of two or more treatment groups) with a total of 10,681 people in our review. There were five different interventions which we call 'supportive interventions': changes to the organisation of nutritional care (13 studies, 3456 people), changes to the feeding environment ( 5 studies, 351 people), modification of the meal profile or pattern (12 studies, 649 people), additional supplementation of meals (10 studies, 6022 people) and home meal delivery systems ( 1 study, 203 people). Monitoring participants over time (follow-up) ranged from 'duration of hospital stay' to 12 months. The comparator groups received 'usual' care. More than half of all participants took part in studies investigating the additional supplementation of meals (for example a protein-energy oral nutritional supplement in addition to the usual diet).

\section{Key results}

It is possible that supportive interventions for enhancing dietary intake in nutritionally vulnerable groups reduce death from any cause (approximately 23 fewer cases of death per 1000 participants in favour of supportive interventions). However, this has to be confirmed by more evidence from high-quality randomised controlled studies. The number of participants experiencing any medical complication did not differ substantially between the supportive interventions and the comparator groups. The same was found for health-related quality of life (which is physical, mental, emotional and social health attributed to health), patient satisfaction, nutritional or energy intake and days spent in hospital. Economic costs were not well investigated. 
Only three studies reported on side effects, describing intolerance to the nutritional supplement (such as diarrhoea or vomiting in 5 of 34 participants) and discontinuation of oral nutritional supplements because of refusal or dislike of taste (567 of 2017 participants).

After analysing 15 studies in 1945 participants we found a beneficial effect of supportive interventions compared with comparators on weight: on average people in the supportive interventions groups increased their weight $0.6 \mathrm{~kg}$ more than people in the comparator groups.

This evidence is up to date as of September 2016.

\section{Quality of evidence}

The overall quality of evidence ranged between moderate to very low, mainly because for most of our outcomes there was only a small number of studies and participants to achieve reliable information, or because risk of bias made results uncertain. However, if some randomised controlled studies with low risk of bias for our patient-important outcomes and a good number of participants were performed, this review could quickly provide good guidance for better health care. 


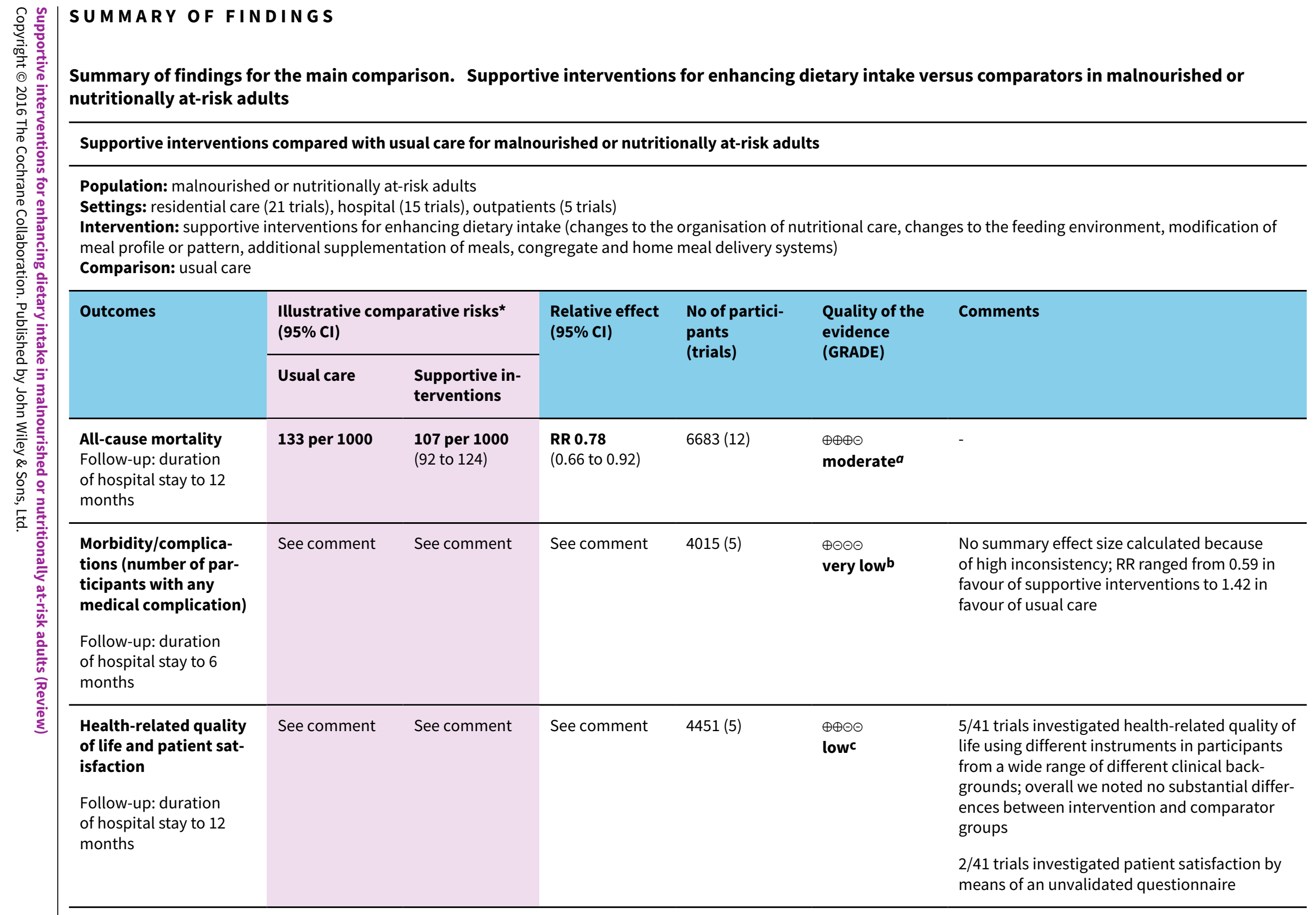

Population: malnourished or nutritionally at-risk adults

Intervention: supportive interventions for enhancing dietary intake (changes to the organisation of nutritional care, changes to the feeding environment, modification of meal profile or pattern, additional supplementation of meals, congregate and home meal delivery systems) Comparison: usual care 


\begin{tabular}{|c|c|c|c|c|c|c|}
\hline $\begin{array}{l}\text { Hospitalisation and } \\
\text { institutionalisation } \\
\text { (days) } \\
\text { Follow-up: } 8 \text { days to } 4 \\
\text { months }\end{array}$ & $\begin{array}{l}\text { The mean hos- } \\
\text { pitalisation } \\
\text { ranged across } \\
\text { control groups } \\
\text { from } 10 \text { days to } \\
40 \text { days }\end{array}$ & $\begin{array}{l}\text { The mean hos- } \\
\text { pitalisation in } \\
\text { the intervention } \\
\text { groups was } \\
\mathbf{0 . 5} \text { days short- } \\
\text { er ( } 2.6 \text { days } \\
\text { shorter to } 1.6 \\
\text { days longer) }\end{array}$ & - & $667(5)$ & $\begin{array}{l}\oplus \ominus \ominus \ominus \\
\text { very lowd }\end{array}$ & $\begin{array}{l}3 / 5 \text { trials with data on hospitalisation were in } \\
\text { the group of trials of 'Changes to the organisa- } \\
\text { tion of nutritional care' }\end{array}$ \\
\hline $\begin{array}{l}\text { Adverse events } \\
\text { Follow-up: } 8 \text { days to } 6 \\
\text { months }\end{array}$ & See comment & See comment & See comment & $4108(3)$ & $\begin{array}{l}\oplus \ominus \ominus \ominus \\
\text { very lowe }\end{array}$ & $\begin{array}{l}\text { Only } 3 / 41 \text { trials reported on adverse events (all } \\
\text { evaluating the impact of supplementation of } \\
\text { meals with oral nutritional supplements); } 1 \text { trial } \\
\text { reported intolerance to the supplement (diar- } \\
\text { rhoea, vomiting) in } 3 / 34 \text { (15\%) of participants. } \\
\text { In another large trial } 565 / 2017 \text { ( } 28 \% \text { ) of stroke } \\
\text { patients stopped taking the oral nutritional } \\
\text { supplements because of refusal or dislike of } \\
\text { taste }\end{array}$ \\
\hline $\begin{array}{l}\text { Nutritional status } \\
\text { (weight change in } \mathbf{k g} \text { ) } \\
\text { Follow-up: } 8 \text { days to } 12 \\
\text { months }\end{array}$ & $\begin{array}{l}\text { The mean } \\
\text { weight change } \\
\text { ranged across } \\
\text { control groups } \\
\text { from }-3.0 \mathrm{~kg} \text { to } \\
+0.3 \mathrm{~kg}\end{array}$ & $\begin{array}{l}\text { The mean } \\
\text { weight change } \\
\text { in the interven- } \\
\text { tion groups was } \\
+\mathbf{0} \mathbf{6} \mathbf{~ k g} \text { higher } \\
(0.2 \mathrm{~kg} \text { to } 1.0 \mathrm{~kg} \\
\text { higher) }\end{array}$ & - & $2024(17)$ & $\begin{array}{l}\oplus \oplus \oplus \ominus \\
\text { moderatef }\end{array}$ & - \\
\hline $\begin{array}{l}\text { Economic costs } \\
\text { Follow-up: duration } \\
\text { of hospital stay to } 12 \\
\text { months }\end{array}$ & See comment & See comment & See comment & $1152(3)$ & $\begin{array}{l}\oplus \odot \odot \odot \\
\text { very lowg }\end{array}$ & $\begin{array}{l}3 / 41 \text { trials evaluated and } 2 / 41 \text { trials reported } \\
\text { some data on economic costs; none of the trials } \\
\text { used accepted health economic methods and } \\
\text { the reported data on both costs and effective- } \\
\text { ness were generally poor }\end{array}$ \\
\hline
\end{tabular}

*The basis for the assumed risk (e.g. the median control group risk across trials) is provided in footnotes. The corresponding risk (and its $95 \%$ confidence interval) is based on the assumed risk in the comparison group and the relative effect of the intervention (and its $95 \% \mathrm{Cl}$ ).

Cl: confidence interval; RR: risk ratio

GRADE Working Group grades of evidence

High quality: Further research is very unlikely to change our confidence in the estimate of effect.

Moderate quality: Further research is likely to have an important impact on our confidence in the estimate of effect and may change the estimate.

Low quality: Further research is very likely to have an important impact on our confidence in the estimate of effect and is likely to change the estimate.

Very low quality: We are very uncertain about the estimate.

*aAssumed risk was derived from the event rates in the comparator groups (usual care) 
owngraded by one level because of risk of bias in several risk of bias domains

bDowngraded by three levels because of risk of bias in several risk of bias domains, serious inconsistency and imprecision

'Downgraded by two levels because of risk of bias in several risk of bias domains, indirectness and few trials investigating health-related quality of life in substantially diverse

trial populations

dDowngraded by three levels because of risk of performance bias and serious imprecision

Downgraded by three levels because of risk of bias in several risk of bias domains, imprecision and general substandard reporting of adverse events in included trials

fDowngraded by one level because of imprecision

gDowngraded by three levels because of risk of bias in several risk of bias domains, imprecision and few trials investigating economic costs with poor reporting, not using accepted

health economic methods 


\section{B A C K G R O U N D}

Malnutrition in patients admitted to hospital was initially recognised in the 1970s (Butterworth 1974 ; McWhirter 1994). In recent years, malnutrition in the community has also been reported (Elia 2009). Whether in the hospital or the community, malnutrition is associated with poor clinical outcome, decreased health-related quality of life and increased mortality (Kubrak 2007; Norman 2008; Stratton 2003).

Malnutrition is both a cause and consequence of ill health (Lean 2008) and its aetiology is complex. It predisposes to illness but is also a consequence of illness (NCCAC 2006), creating a vicious, self-perpetuating cycle of malnutrition and infection (Scrimshaw 2003). People who are undernourished on admission to hospital, who do not receive adequate nutritional care, experience decline in their nutritional status (McWhirter 1994). While in hospital, the reasons for further poor intakes and subsequent weight loss may include temporary starvation for medical procedures, difficulty in feeding, lack of nursing supervision during mealtimes, depression, unpalatable foods and disease- or drug-induced anorexia (Kelly 2000; Lennard-Jones 1992). At home, in addition to the effects of illness and its management, sub-optimal nutritional status may be due to practical challenges, such as lack of transport, difficulties in grocery shopping, or difficulties utilising cooking facilities, resulting in diets of poor nutritional quality. Social and psychological issues also have a significant impact. The factors that contribute to malnutrition in hospital and community patients have been described extensively elsewhere (Lennard-Jones 1992; NCCAC 2006).

Nutrition intervention and treatment of malnutrition has been recommended in clinical guidelines from many countries based on associations between improved dietary intake and nutritional status, health-related quality of life and functional outcomes (Mueller 2011; NCCAC 2006). Therefore, it is recommended that at the first sign of malnutrition or risk of malnutrition, a full nutritional assessment and appropriate nutritional intervention should follow (Mueller 2011; NCCAC 2006). As the causes of malnutrition are multifactorial, the interventions designed to treat malnutrition are likely to be complex. This merits an understanding of the multidimensional causes of malnutrition and the complex support strategies needed across a range of healthcare services from the strategic policy level down to the individual feeding of a patient (Weekes 2009).

\section{Description of the condition}

Despite the absence of universally accepted diagnostic criteria, a widely quoted definition describes malnutrition as the nutritional state in which an energy, protein or nutrient deficiency, excess or imbalance leads to adverse effects on body or tissue form (body shape, size and composition) and function, as well as clinical outcome (Elia 2003). The recently convened International Guideline Consensus Committee categorised malnutrition as, "starvationrelated malnutrition" in cases of chronic starvation in the absence of inflammation, "chronic disease-related malnutrition" where there is chronic but mild-to-moderate inflammation and, "acute disease or injury-related malnutrition" where there is acute severe inflammation (Jensen 2010). While this provides a useful aetiological classification of malnutrition and recognises the effect of illness on nutritional status, there remain no clear criteria for how each category might be identified in practice. Nutrition screening is often used to detect risk factors known to be associated with nutritional complications (McMahon 2000) such as recent, unintentional weight loss; inadequate food intake; disease-related anorexia; low body weight, body mass index (BMI) or lean body mass; in order to decide whether a full nutritional assessment is indicated (Elia 2003). Nutrition screening tools commonly employ a standard pro forma to determine nutritional risk. The included parameters are intended to determine whether an individual is nutritionally at risk on the basis of a score, which determines the course of action (Green 2006; Jones 2002). Many tools suggest suitable action plans that may involve nutritional intervention. Nutritional assessment is a more comprehensive investigation including anthropometric measurements, biochemical tests, clinical examination and dietary intake monitoring, used to determine whether an individual is malnourished or likely to become malnourished (at risk of malnutrition) (Corish 2000a; McMahon 2000). Nutritional assessment is usually followed by appropriate nutritional intervention (Corish 2000a; McMahon 2000).

The absence of clear and universally accepted criteria for the diagnosis of malnutrition further complicates the interpretation of prevalence data and intervention trials. Major classic and more recent trials that assessed the prevalence of malnutrition in hospitals have estimated a prevalence of between $11 \%$ and $50 \%$ depending on the criteria used (Bistrian 1974; Corish 2000a; Corish 2000b; Edington 2000; Hill 1977; Kelly 2000; McWhirter 1994; Naber 1997). The variation in reports of prevalence result largely from differences in the definitions used to identify malnutrition across trials. In 2008, the nutrition screening week carried out by the British Association for Parenteral and Enteral Nutrition (BAPEN), which uses a standardised tool to assess nutritional risk status, demonstrated that malnutrition was present in nearly a third of people admitted to hospital, in just over a third of people admitted to care homes and in a fifth of people admitted to mental health units (Elia 2009). Furthermore, it has been estimated that at any given time over three million people in the UK are thought to be malnourished or at risk of malnutrition with the vast majority of these (93\%) living at home (Elia 2009). In Australia, a survey that used a different nutrition screening tool to screen 3122 participants in the acute hospital setting, revealed that $41 \%$ of participants were "at risk" of malnutrition, with an overall prevalence of malnutrition of 32\% (Agarwal 2011).

The clinical consequences of malnutrition are believed to include reduced muscle strength; failure of the respiratory, thermoregulatory, pancreatic, gastrointestinal, mental, endocrine, and cardiovascular systems; as well as impaired wound healing and poor clinical outcomes from surgical procedures or illness (Allison 2000; Corish 2000a; Lennard-Jones 1992). Wounds that heal more slowly become much more vulnerable to infection. Immune function is impaired, compounding constraints on the body from other disease states, constituting a much reduced resistance to infection (Corish 2000a). Respiratory muscle wasting may also predispose to infections if patients are unable to cough and expectorate effectively (Lennard-Jones 1992). Pressure sores may develop as mobility is reduced (Lennard-Jones 1992) and as the body becomes thinner and wasted. Arguably, the effects of malnutrition on the musculoskeletal system extend beyond the gain or loss of lean body tissue, but may incur metabolic changes in cellular electrolytes including calcium accumulation, which may prevent optimal muscle function (Jeejeebhoy 1986). Furthermore, 
excretory systems may fail to regulate body sodium-water balance efficiently and may result in excess fluid retention and oedema (Allison 2000), which has reportedly been detected in $17 \%$ of malnourished people admitted to hospital (Weekes 1999). As disease further impinges on appetite (Allison 2000), malnutrition will progress and the clinical implications aforementioned will occur much more quickly in ill people than in healthy individuals (Corish 2000a).

In addition to the clinical and social consequences, the economic impact of malnutrition is considerable. The increasing costs have become an economic burden for healthcare systems in many countries. Recent data from the UK suggest that malnutrition costs in excess of GBP 7.3 billion each year (EURO 8.74 billion/ year - December 2011 conversion) (DOH 2007; Russell 2007). Poor clinical outcomes, such as extended hospital stays, increased medical complications, reduced health-related quality of life and slow disease recovery, all contribute to rising hospital and home care costs (Gallagher 1996; Russell 2007; Stratton 2003). Malnourished patients stay in hospital for longer, are three times more likely to develop complications during surgery and have a higher mortality than adequately nourished patients ( $\mathrm{DOH}$ 2007). Furthermore, those considered at risk of malnutrition are much more likely to require home healthcare services after discharge from hospital than those considered not at risk (Chima 1997). Malnutrition in the community has also been shown to increase the need for healthcare resources such as general practitioner (GP) visits, hospital admissions and new prescriptions, in addition to contributing to an increased risk of mortality (Martyn 1998). Therefore, if healthcare economics is considered, an undernourished patient imposes a greater economic burden on health services than a patient whose nutritional status is well maintained (Lennard-Jones 1992).

\section{Description of the intervention}

This review seeks to determine whether effective clinical management of malnutrition in both hospital and community settings requires more than just the provision of nutrients, dietary advice, or a combination, and whether additional strategies to support these existing approaches to ensure overall nutritional care is optimal, is worthy of consideration. The specific types of interventions considered are listed in Table 1. Related interventions include the sole use of oral nutritional supplements, dietary counselling or strategies, or a combination to manage malnutrition.

Guidelines exist for the identification, regular monitoring and initiation of nutritional support in individuals who may be malnourished or at nutritional risk. These include UK clinical guidelines for nutritional screening and support in adults (NCCAC 2006), Essence of Care benchmarks for food and nutrition from the UK Department of Health (DOH 2003), and the American Society for Parenteral and Enteral Nutrition (ASPEN) guidelines on nutrition screening, assessment and intervention in adults (Mueller 2011).

The strategies most frequently used to treat malnutrition in individuals requiring nutritional support aim to increase energy and nutrient intake by means of the following.

- Dietary counselling - provision of nutritional advice to increase nutrient intake, requiring an individual to understand and act upon instructions given. This approach may include providing advice on food fortification, to increase the energy density of foods without increasing quantity, or dietary fortification, to increase the energy density of the diet by adding extra snacks or drinks between meals.

- Oral nutritional supplements - available in either liquid or solid forms. These usually provide a mixture of macro- and micronutrients and may be nutritionally complete in a specified volume and are often available in the form of commercial supplement products.

- Artificial nutrition support - includes enteral tube feeds and parenteral nutrition that are used when oral intake is not possible.

The efficacy of nutritional support interventions has been the subject of much previous research but so far has focused primarily on the use of oral nutritional supplements, which may be applicable to only a minority of people (Weekes 2009). There are more than 20 systematic reviews in the literature of oral nutritional supplement-based interventions in the management of malnutrition (Stratton 2007). The findings are variable with some reviews showing clinical and nutritional benefits (Stratton 2007). However, these findings are by no means consistent and the patient groups most likely to benefit from this type of intervention remain to be characterised (Stratton 2007). Despite this, there has been a consistent trend to use oral nutritional supplements in clinical practice but the high cost implications of this approach, especially in the community as recently highlighted in a UK report (LPP 2009), makes the consideration of alternative approaches worthwhile. There has been an increased focus on the routine provision of food and drink as part of nutritional care since the 10 key characteristics of good nutritional care in hospital were published (COE 2003). Forty-five trials have examined the role of food-based interventions with or without oral nutritional supplements in the management of poor dietary intake (Baldwin 2011). The findings suggested that although dietary counselling may result in improvements in weight, body composition and muscle function, trials were heterogeneous and of variable quality with no evidence of benefit on mortality (Baldwin 2011). These trials have concentrated on interventions that rely on the patient receiving and acting on instructions to enhance their nutritional intake (i.e. dietary counselling). Despite the body of clinical evidence supporting the appropriate use of oral nutritional supplements and previous research around dietary counselling, whether additional supportive interventions are clinically effective in the management of malnutrition or the risk of malnutrition, remains unknown.

The Council of Europe and the UK Department of Health highlighted the importance of overall nutritional care including, among other supportive initiatives: mandatory nutritional screening, adequate provision of food and drink, oral supplements, modified diets, assistance with feeding and changes to the dining environment (COE 2003; DOH 2007). Such interventions have been incorporated into guidelines and healthcare policies and aim to improve nutritional intake by modifying aspects of food provision (e.g. the use of protected mealtimes, red tray initiatives (to identify those requiring mealtime assistance) and feeding assistance) or by adjusting the portion size and nutrient content of foods and enhancing the flavour, however, evidence of benefit of such initiatives is lacking. 


\section{Adverse effects of the intervention}

The possible adverse effects of the supportive nutritional care interventions considered in this review may include but are not limited to the following events: provision of incorrect nutritional supplement, provision of incorrect between-meal snacks, gastrointestinal effects due to intolerance of supplements/ extra snacks/drinks (e.g. bloating, vomiting or diarrhoea), potential accidents occurring as a result of the intervention such as a patient falling on the way to a dining area in a change of dining environment intervention, inappropriate moving and handling by untrained staff trying to obtain a weight or height measure, inappropriate screening or intervention (e.g. during end of life).

\section{How the intervention might work}

As recommended in the PRISMA statement (Liberati 2009), a conceptual framework highlighting the participants, interventions, comparisons, outcomes and trial design (PICOS) considered for this review, is illustrated (Figure 1 ). 


\section{Figure 1.}
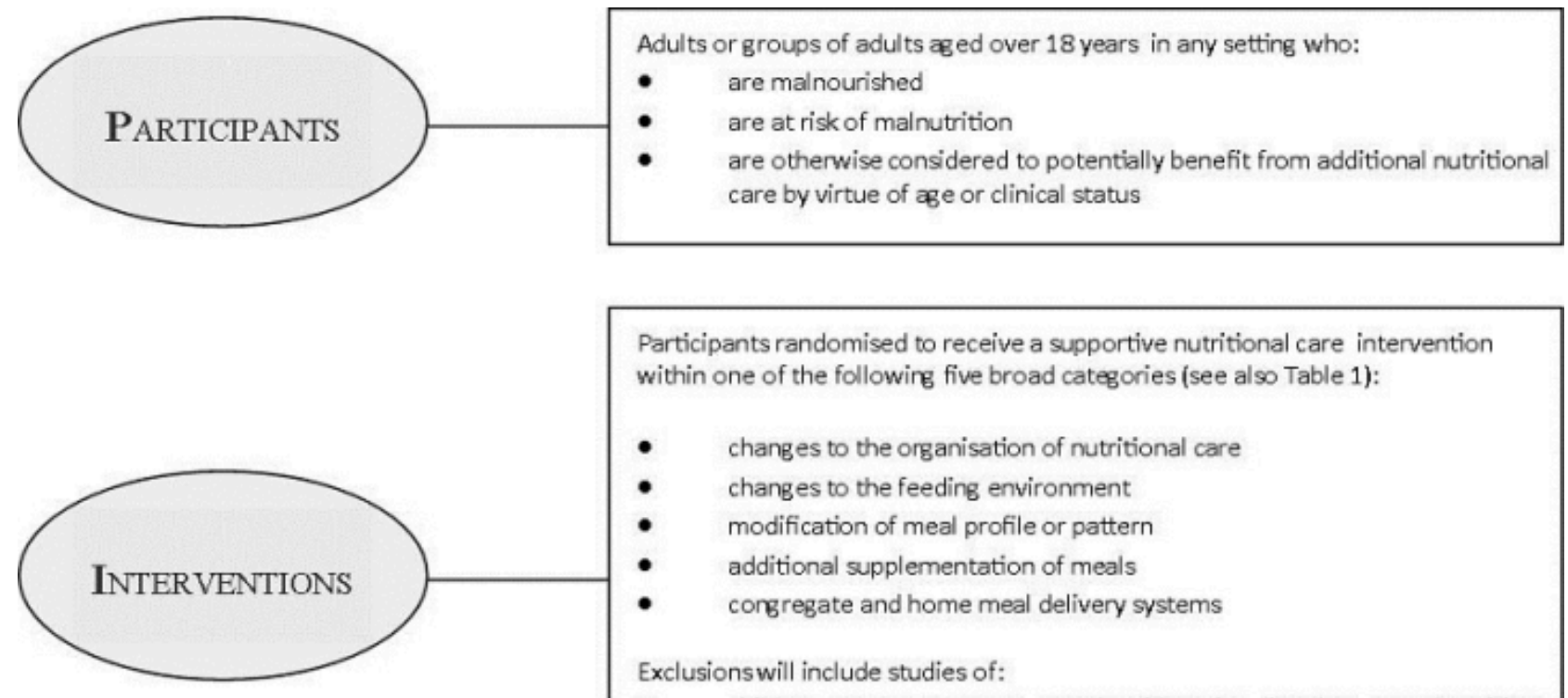

Participants randomised to receive a supportive nutritional care intervention within one of the following five broad categories (see also Table 1):

- changes to the organisation of nutritional care

- changes to the feeding environment

- modification of meal profile or pattern

- additional supplementation of meals

- congregate and home meal delivery systems

Exclusionswill include studies of:

- children, pregnant women, people with eating disorders, or malnutrition in conditions of food insufficiency and poverty

- $\quad$ artificial nutrition support via a non-oral route (i.e. enteral tube feeding and parenteral nutrition)

- individualised dietary counselling

- individually prescribed oral nutritional supplements

- healthy volunteers

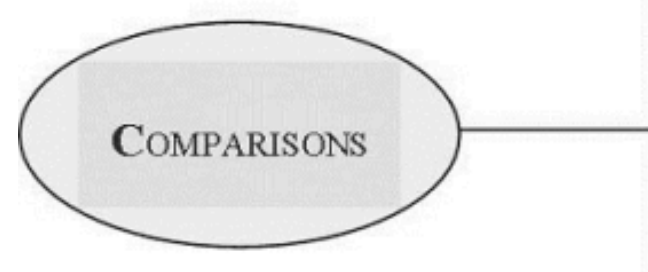

Participants randomised to receive routine nutritional care which may include:

- non-systematic referral to dietitian for nutritional assessment, dietary advice and/or oral nutritional supplements

- $\quad$ inconsistent nutritional screening

- standard hospital/institution meal service on a ward or in other institutionalised setting

- no change to the feeding environment

- unmodified, non-supplemented meals

- no assistance with meals in the community

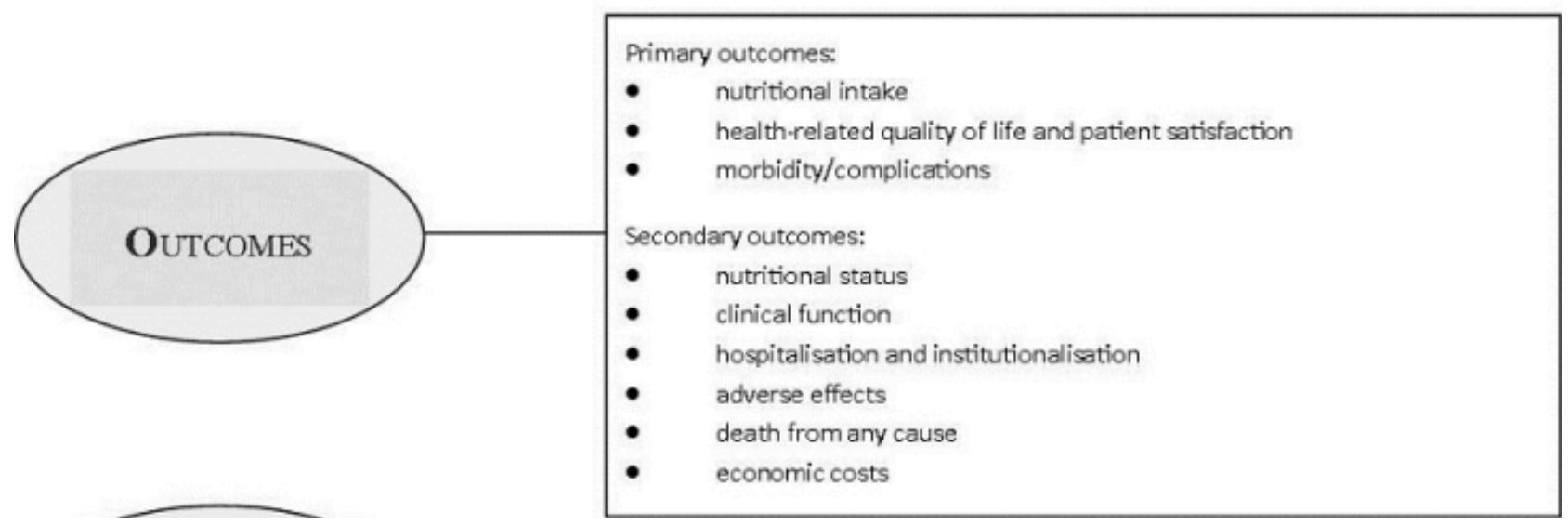


Figure 1. (Continued)

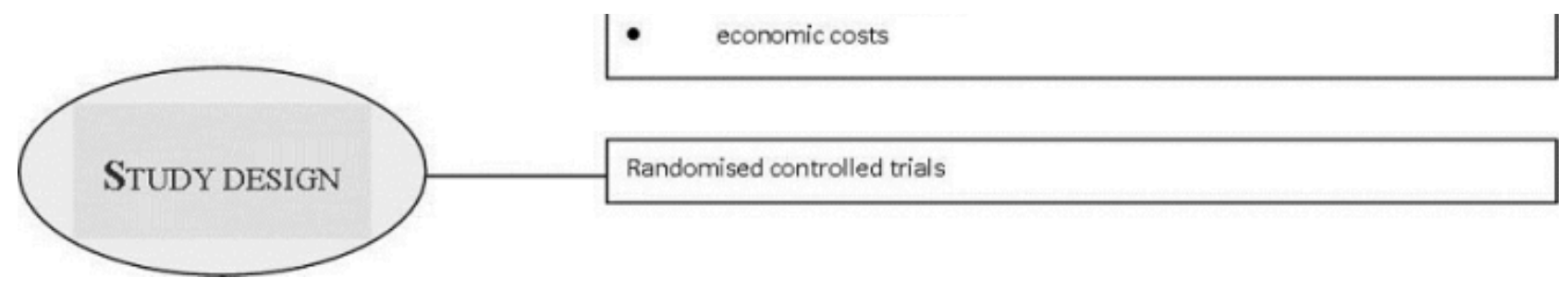

The treatment of malnutrition aims to reverse its effects, including the physical and functional impairments, and the provision of appropriate nutritional care may involve several approaches. The factors that influence our experiences with food are complex and nutritional care interventions aimed at the management of malnutrition or nutritional risk may need to address more than the provision of energy (calories). The biological and symbolic dimensions of food are inseparable and a socio-anthropological perspective suggests an intimate yet dynamic relationship between consumption of food and perceptions of self (Lupton 1996). The meaning of food extends beyond its mere nutritive value as it can have a tremendous impact on a person's sense of independence, self-esteem, well-being and health-related quality of life, especially in elderly people (Donini 2003). Indeed, experiences with food have important implications for the emotional and psychological well-being of an individual that sit within a traditional, cultural, socioeconomic and religious context and ultimately determines our food preferences (Donini 2003; Khan 1981; Lupton 1996). In severe illness, coping mechanisms, sense of body image, value of social networks and support, and personal symbolism may all be affected and food may take on new meaning (McQuestion 2011). Overall, this represents a challenge to health professionals and merits a deeper understanding of what really impacts on our experiences with food. Taking this into account, interventions that enhance the food experiences of malnourished individuals or those at risk of malnutrition by supporting their ability to take the intervention, thereby improving compliance, should theoretically result in greater dietary intakes and improved outcomes. Furthermore, the benefits of such interventions may extend beyond the conventional clinical, nutritional or functional outcomes and could conceivably also improve patient-satisfaction and perceived health-related quality of life. Indeed, following improvements in nutritional intake there may also be psychological and social benefits in individuals who are malnourished or at risk of malnutrition (NCCAC 2006). To summarise the mode of action, supportive nutritional care interventions should theoretically increase intake of micro- and macro-nutrients and, in turn, improve the nutritional status and clinical function of nutritionally atrisk individuals. By this, mortality, morbidity and hospitalisation are expected to be lowered. Considering the beneficial effects on physical health and the symbolic dimensions of food, healthrelated quality of life should also improve.

\section{Why it is important to do this review}

A Cochrane systematic review of protein and energy supplementation in individuals over 65 years at risk from malnutrition contains 62 trials with a total of 10,187 randomised participants and the authors concluded that supplementation led to small but consistent weight gain in older people, and reductions in mortality in those who were undernourished (Milne 2009). There was no evidence of benefit to complications, functional status or length of hospital stay (Milne 2009). Interventions considered focused primarily on dietary supplementation with commercial sip feeds, milk-based supplements and via the fortification of normal food sources (Milne 2009), rather than the array of supportive nutritional care interventions of interest to this review. In addition, the review included both randomised and quasirandomised trials (e.g. allocation by alternation, day of week, date of birth) (Milne 2009). It is acknowledged that the complex nature of the interventions in this area may result in trials that lack robust design and their inclusion may best represent the body of evidence available. However, meaningful conclusions may be more difficult to decipher, and therefore this systematic review of purely randomised controlled trials will better highlight the research needs and knowledge gaps in this area. Furthermore, a wider range of interventions and trials including adults of all ages have been considered in this review.

There is an urgent need to identify effective strategies for the management for malnourished people in hospitals and other health and social care settings. Not only has this been highlighted in reports from the Council of Europe (COE 2003) and within the UK by the Department of Health ( $\mathrm{DOH}$ 2007), but also by professional bodies such as the Royal College of Nursing, the British Association for Parenteral and Enteral Nutrition (BAPEN) and patient-focused organisations such as Age UK (BAPEN 2009; RCON 2008). Numerous strategies aimed at influencing nutritional management and improving the provision of nutritional care in hospitals, care homes and other health and social care settings, have been adopted and incorporated into national policies and international guidelines. Additionally, in the UK, protected mealtimes and the use of red trays have been rolled out across the National Health Service very recently, and interventions applicable across a range of healthcare settings, such as the use of feeding assistance, adjusting the portion size and nutrient content of foods and enhancing food flavours, are increasingly being used. Such service developments have received widespread support by local and national organisations and government. There has been a consistent trend to recommend the implementation of policies designed to influence nutritional care and the environment in which nutrition is provided, without a synthesis of the evidence of potential benefits or harms of such interventions. Crucially, the incorporation of such initiatives into usual care has implications for the staffing and funding of healthcare as well as the potential need for additional training across services. As yet there has been no synthesis of evidence to support the potential benefits of their implementation. Furthermore, a supportive multidisciplinary team approach is necessary in the provision of adequate nutritional care (Jefferies 2011). Given the widespread prevalence of malnutrition and with so many at risk, the potential impact of this systematic review in terms of informing the nutritional management of 
patients is considerable and therefore, the need for this review was paramount.

Two literature reviews examined various supportive nutritional care interventions (Silver 2009; Weekes 2009) but neither was systematic and both presented a narrative synthesis without meta-analysis. Furthermore, the review by Weekes and colleagues (Weekes 2009) included non-randomised trials and searched only electronic sources, while the review by Silver (Silver 2009) considered only trials in older adults. Despite their usefulness in presenting some of the available literature in this area, the true effect of supportive interventions to improve dietary intake by modifying the nutrient content of foods served or aspects of the food service system or environment remains unknown. Therefore, this review represents a first systematic attempt to bring together evidence on the impact of supportive interventions on nutritional, clinical, economic and patient-centred outcomes.

\section{O B JECTIVES}

To assess the effects of supportive interventions for enhancing dietary intake in malnourished or nutritionally at-risk adults.

\section{METHODS}

\section{Criteria for considering studies for this review}

\section{Types of studies}

We included randomised controlled clinical trials (RCTs).

\section{Types of participants}

Adults (aged over 18 years) who were malnourished, judged to be at nutritional risk or otherwise would potentially benefit from improved nutritional care. The population is therefore described as nutritionally vulnerable.

\section{Diagnostic criteria (malnourished or nutritionally at-risk adults)}

The term malnutrition used in this review refers to under-nutrition, considered to be the state of poor nutritional status as a result of inadequate nutrient intake or metabolic impairment as well as the state of increased nutritional risk and imminent malnutrition (Corish 2000a; Reilly 1995).

The Malnutrition Universal Screening Tool (MUST) published by BAPEN (Elia 2003), as well as clinical guidelines in the UK and Europe published by the European Society for Parenteral and Enteral Nutrition (ESPEN) (Volkert 2006) and the National Institute for Health and Care Excellence (NICE) (NCCAC 2006), allow identification of malnourished individuals and those at risk of malnutrition in clinical practice and may be used to classify trial participants. These criteria are:

\section{Malnourished}

NICE (NCCAC 2006)

- Body mass index (BMI) below $18.5 \mathrm{~kg} / \mathrm{m}^{2}$

- Unintentional weight loss greater than $10 \%$ within the last three to six months

- BMI below $20 \mathrm{~kg} / \mathrm{m}^{2}$ and unintentional weight loss greater than $5 \%$ within the last three to six months

ESPEN (Volkert 2006)
- $5 \%$ unintentional weight loss in last three months and BMI below $20 \mathrm{~kg} / \mathrm{m}^{2}$

- $10 \%$ unintentional weight loss in last six months and BMI below $20 \mathrm{~kg} / \mathrm{m}^{2}$

\section{At risk of malnutrition}

NICE (NCCAC 2006)

- Have eaten little or nothing for more than five days, are likely to eat little or nothing for the next five days or longer, or both

- Have a poor absorptive capacity, have high nutrient losses, have increased nutritional needs from causes such as catabolism, or a combination

\section{ESPEN (Volkert 2006)}

- Loss of appetite

- Reduced dietary intake

- Physical or psychological stress

MUST (Elia 2003)

- Current acute illness plus no (or likely to be no) nutritional intake for more than five days

In the absence of clear, internationally accepted diagnostic criteria for clinical malnutrition, in many instances a health professional's decision to initiate dietetic referral for nutritional assessment or a clinician's decision to commence nutritional intervention is based on subjective criteria and clinical judgement (McCarron 2010). It was assumed therefore, that participants recruited to intervention trials were judged by the researcher to be malnourished or at risk of malnutrition, or otherwise had the potential to benefit from improved nutritional care on the basis of their clinical background or age.

\section{Types of interventions}

\section{Intervention}

Interventions that aimed to enhance food intake by improving either the meal itself (e.g. food fortification), aspects of the mealtime environment (e.g. enhancement of the eating environment), aspects of meal delivery, supplementation of meals or indirect supportive strategies (e.g. training of staff or carers). The strategies anticipated prior to searching included the examples listed within the five categories shown in Table 1. However, we recognised that it may become necessary to create additional categories as necessary following searching.

A previous systematic review (Baldwin 2011) included trials of interventions based on dietary counselling that required a person to receive instruction on food modification, oral nutritional supplements or both and have the ability and willingness to act on the instructions in order to enhance their nutritional intake. Although this review is closely related to the previous review, we planned to exclude trials where dietary counselling or oral nutritional supplements, or both were offered on an individualised basis. This review only considered food-based or oral nutritional supplement interventions when they were provided as an institution-led intervention without the patient needing to understand and act on instructions to take the additional items (e.g. offering snacks or supplements routinely 
to frail elderly people in an institutional setting, or the use of organisational structures to support the delivery of oral nutritional supplements). The inevitable overlap with reviews of oral nutritional supplements in the management of malnutrition is noted, but the inclusion of such trials in this review contributes to a more precise understanding of the benefits to be derived from these products.

\section{Comparator}

All interventions were compared with usual care.

\section{Summary of specific exclusion criteria}

We excluded the following intervention trials from this review.

- Trials in children, pregnant women, people with eating disorders or malnutrition in conditions of food insufficiency and poverty. We have excluded these trials as malnutrition in such cases results from different aetiology, and the types of interventions and responses to such interventions also differ.

- Trials of artificial nutrition support via a non-oral route (i.e. enteral tube feeding and parenteral nutrition).

- Trials of individualised nutritional support including either dietary counselling (i.e. where the individual was required to understand and act upon specific nutritional advice, which is most likely to occur in the outpatient setting). In cases where dietary advice was provided in combination with a supportive intervention, we have only included the trial if it was possible to evaluate the impact of the supportive intervention separately.

- Trials of individually prescribed oral nutritional supplements.

- Trials in healthy volunteers.

\section{Types of outcome measures}

We recorded the following outcome measures as change from baseline to end of intervention unless otherwise stated.

\section{Primary outcomes}

- Nutritional intake (actual or percentage change in macro- and micronutrient intake)

- Health-related quality of life (evaluated by validated scores) and patient satisfaction

- Morbidity/complications (number of participants with medical complications)

\section{Secondary outcomes}

- Nutritional status (change in weight, body mass index (BMI), mid-upper arm circumference (MUAC), triceps skin-fold thickness (TSF) or as otherwise reported)

- Clinical function (change in clinical functional status (e.g. skeletal muscle strength), respiratory and cardiac function, cognitive and behavioural function, activities of daily living)

- Hospitalisation and institutionalisation

- Adverse events

- All-cause mortality

- Economic costs

\section{Timing of outcome measurement}

We extracted data on outcomes measured in each trial from baseline to the end of the intervention period. For trials with follow- up periods that extended beyond the end of the intervention, we also extracted data at the end of intervention to the point of final follow-up. From experience of a previous review of dietary advice with or without oral nutritional supplements for disease-related malnutrition in adults (Baldwin 2011) we anticipated that the length, intensity and type of intervention would vary considerably in this current review, given its wider scope. We did not, therefore, establish lengths of intervention and only grouped interventions by time point if a sufficient number of trials was identified to permit this.

\section{Summary of findings}

We have presented a 'Summary of findings' table to report the following outcomes, listed according to priority.

- All-cause mortality

- Morbidity/complications

- Health-related quality of life and patient satisfaction

- Hospitalisation and institutionalisation

- Adverse events

- Nutritional status

- Economic costs

Because of lack of data and substantial clinical and methodological heterogeneity we only performed meta-analyses on all-cause mortality, number of participants with complications and nutritional status (weight change).

\section{Search methods for identification of studies}

\section{Electronic searches}

We searched the following sources from inception of each database to the specified date and placed no restrictions on the language of publication.

- Cochrane Library (14 September 2016).

- Ovid Epub Ahead of Print, In-Process \& Other Non-Indexed Citations, Ovid MEDLINE(R) Daily and Ovid MEDLINE(R) (1946 to 14 September 2016).

- Embase (to March 2013).

- AMED (to March 2013).

- British Nursing Index (to March 2013).

- CINAHL (to March 2013).

- SCOPUS (to May 2013).

- ISI Web of Science (to March 2013).

- ClinicalTrials.gov (14 September 2016).

- World Health Organization (WHO) ICTRP (International Clinical Trials Registry Platform - http://apps.who.int/trialsearch/) (14 September 2016)

During the first round of electronic searches, we searched databases for all trials published up until the end of October 2011. During the second round of electronic searches, we searched databases for trials published between November 2011 and the end of March 2013 (May 2013 for SCOPUS only). We used identical search strategies in both the first and second round of searches. We carried out a third round of electronic searches prior to publication, when we used a revised search strategy to search the Cochrane Library, Ovid MEDLINE, ClinicalTrials.gov and WHO ICTRP. We carried out revised searches of the Cochrane Libary and Ovid MEDLINE from 1 
January 2013 to 14 September 2016. We searched ClinicalTrials.gov and the ICTRP from inception to 14 September 2016.

For detailed search strategies please see Appendix 1 and Appendix 2.

\section{Searching other resources}

We searched the references lists of included trials and (systematic) reviews, and meta-analyses to identify additional trials. We also searched the conference proceedings of relevant professional bodies and associations (British Dietetic Association, BAPEN and Royal College of Nursing) for the 10-year period 2001 to 2011.

\section{Data collection and analysis}

\section{Selection of studies}

In order to identify trials to be assessed further, two review authors (MG and CEW) independently scanned the abstract, title or both for every record retrieved according to the inclusion criteria for the first round of searches. For the second round of searches, MG and CB independently scanned the abstract, title or both for every record retrieved according to the inclusion criteria, as before. For the third round of searching, CB and Bernd Richter (The review group editor) scanned titles and abstracts. We obtained all potentially relevant articles as full text and the three review authors (MG, CB and CEW) independently assessed their eligibility using a standardised trial eligibility form. Where there were differences in opinion, we resolved them by discussion among the three authors and made a decision by consensus. If resolving disagreement was not possible, we added the article to those 'awaiting assessment' and contacted the trial authors for clarification. We marked trials where we had not reached a primary consensus and if we included them later on, we planned to subject them to a sensitivity analysis. We listed excluded trials in the 'Characteristics of excluded studies' table along with the reasons for their exclusion. We present an adapted PRISMA flow-diagram of trial selection (Liberati 2009).

\section{Data extraction and management}

For trials that fulfilled the inclusion criteria, two review authors (CB, CEW) abstracted relevant population and intervention characteristics using modified versions of standard data extraction sheets from the CMED Group which incorporated some adaptations from the data collection form used in a previous review by two of the review authors (Baldwin 2011). Data are reported as shown in Table 2; Table 3; Table 4; Table 5; Table 6; Table 7; Table 8; Table 9; Table 10; Table 11; Table 12; Table 13; Table 14; Table 15; Table 16; Table 17; Table 18; Table 19; Table 20; Table 21; Table 22; Table 23; Table 24; Table 25; Table 26 and Appendix 3; Appendix 4; Appendix 5; Appendix 6; Appendix 7; Appendix 8; Appendix 9; Appendix 10. The third review author acted as an arbiter in case of disagreement.

We sent an email request to authors of included trials to enquire whether they were willing to answer questions regarding their trials. Appendix 11 shows the results of this survey. Thereafter, we sought relevant missing information on the trial from the trial authors of the article, if required.

\section{Dealing with duplicate publications}

In the case of duplicate publications and companion papers of a primary trial, we have tried to maximise yield of information by inclusion of and simultaneous evaluation of all available data.

\section{Assessment of risk of bias in included studies}

Two review authors ( $C B$ and $C E W$ ) assessed each trial independently. We resolved possible disagreements by discussion amongst the three authors and made a judgement based on consensus.

We assessed risk of bias using the Cochrane tool for assessing risk of bias (Higgins 2011a; Higgins 2011b). We used the following risk of bias criteria.

- Random sequence generation (selection bias)

- Allocation concealment (selection bias)

- Blinding (performance bias and detection bias), separated for blinding of participants and personnel and blinding of outcome assessment

- Incomplete outcome data (attrition bias)

- Selective reporting (reporting bias)

- Other bias

We assessed risk of bias for each component of each trial as 'low risk', 'high risk' or 'unclear risk' as described in the Cochrane Handbook for Systematic Reviews of Interventions (Higgins 2011a).

\section{Measures of treatment effect}

We expressed dichotomous data as risk ratios (RRs) with 95\% confidence intervals $(\mathrm{Cls})$ and continuous data as mean differences (MDs) with 95\% Cls.

\section{Unit of analysis issues}

We planned to take into account the level at which randomisation occurred, such as cross-over trials, cluster-randomised trials and multiple observations for the same outcome. For cross-over trials data had to be available from baseline to the end of phase 1 of the cross-over trial to be included in meta-analyses. The cross-over design as such was not feasible for our research question because of anticipated substantial carryover effects.

We could not recalculate data taking into account the design effect for cluster-RCTs because we did not have reliable information about intracluster correlation coefficients for our substantial heterogeneous populations in the included trials. Therefore, we did not establish meta-analyses by using both parallel and cluster-RCTs but excluded the cluster-RCTs from all meta-analyses.

\section{Dealing with missing data}

Where feasible, we obtained relevant missing data from study authors. We investigated attrition rates, for example number of dropouts, losses to follow-up and withdrawals, and critically appraised issues of missing data and imputation methods (e.g. lastobservation-carried-forward (LOCF)).

\section{Assessment of heterogeneity}

In the event of substantial clinical, methodological or statistical heterogeneity, we did not report trial results as the pooled effect estimate in a meta-analysis. We identified heterogeneity (inconsistency) through visual inspection of the forest plots and by using a standard $\mathrm{Chi}^{2}$ test with a significance level of $\alpha=0.1$. In view of the low power of this test, we also considered the $I^{2}$ statistic, which quantifies inconsistency across trials to assess the impact of heterogeneity on the meta-analysis (Higgins 2002; Higgins 2003); 
where an $I^{2}$ statistic of $75 \%$ or more indicates a considerable level of heterogeneity (Deeks 2011).

When we found heterogeneity, we attempted to determine possible reasons for it by examining individual trial and subgroup characteristics.

\section{Assessment of reporting biases}

If we included 10 trials or more investigating a particular outcome and intervention, we planned to use funnel plots to assess small study effects. Several explanations can be offered for the asymmetry of a funnel plot, including true heterogeneity of effect with respect to trial size, poor methodological design (and hence bias of small trials) and publication bias. Therefore we interpreted results carefully (Sterne 2011).

\section{Data synthesis}

Prior to undertaking any data synthesis, two authors (CB, CEW) considered the clinical heterogeneity of the trials. The likelihood of clinical heterogeneity amongst trials was judged to be high in many cases, as trials were in populations with widely different clinical backgrounds, conducted in different healthcare settings, and despite some grouping of similar interventions, involved interventions that varied considerably. We undertook data synthesis, therefore, for some outcome measures only, by means of a random-effects model.

\section{Quality of evidence}

We presented the overall quality of the evidence for each outcome according to the GRADE approach, which takes into account issues not only related to internal validity (risk of bias, inconsistency, imprecision, publication bias) but also to external validity such as directness of results. We presented a summary of the evidence in Summary of findings for the main comparison. This provides key information about the best estimate of the magnitude of the effect, in relative terms and absolute differences, for each relevant comparison of alternative management strategies, numbers of participants and trials addressing each important outcome and the rating of the overall confidence in effect estimates for each outcome. We created the 'Summary of findings' table based on the methods described in the Cochrane Handbook for Systematic Reviews of Interventions (Schünemann 2011) by means of the Review Manager (RevMan) table editor (RevMan 2014). We included the Appendix 11 'Checklist to aid consistency and reproducibility of GRADE assessments' (Meader 2014) to help with standardisation of the 'Summary of findings' tables. We presented the results for the outcomes as described in the Types of outcome measures section. If meta-analysis was not possible, we presented results in a narrative format in the 'Summary of findings' table. We justified all decisions to downgrade the quality of trials using footnotes, and we made comments to aid the reader's understanding of the review where necessary.

\section{Subgroup analysis and investigation of heterogeneity}

We undertook the following subgroup analysis.

- Intervention category (e.g. changes to the organisation of nutritional care, changes to the feeding environment, modification of meal profile or pattern, additional supplementation of meals, congregate and home meal delivery systems)
Insufficient data were available to undertake the following subgroup analyses.

- Intervention format (e.g. interventions given to individuals or groups of individuals)

- Baseline nutritional status (e.g. judged to be malnourished or at risk of malnutrition)

- Mean age of participants (e.g. below 65 years and 65 years or over)

- Intervention setting (e.g. home, hospital, long-term care facility, other community setting)

- Intervention duration (e.g. short term (less than 3 months), medium term (3 to 6 months) or long term (above 6 months))

- Intensity of intervention (e.g. number of visits/consults; considerations will be given to a post hoc analysis if sufficient data are available, as the intensity of intervention is very likely to differ according to care setting)

- Effects beyond the cessation of intervention (e.g. maintenance of weight gain, continued improvements in health-related quality of life)

- Change in outcome versus no change in outcome for nutritional status and intake

\section{Sensitivity analysis}

We planned to perform sensitivity analyses to explore the influence of the following factors (when applicable) on effect sizes by restricting the analysis to the following.

- Published trials

- Taking into account risk of bias, as specified in the Assessment of risk of bias in included studies section

- Very long or large trials to establish the extent to which they dominate the results

- Trials using the following filters: diagnostic criteria, imputation, language of publication, source of funding (industry versus other), or country

We also planned to test the robustness of the results by repeating the analysis using different measures of effect size (RRs, ORs etc.) and different statistical models (fixed-effect and random-effects models).

Due to lack of data we only performed sensitivity analyses on some risk of bias.

\section{RE S U L T S}

\section{Description of studies}

See: Characteristics of included studies; Characteristics of excluded studies; Characteristics of studies awaiting classification.

\section{Results of the search}

The electronic searches identified 29,155 records. An additional 1107 records were identified from searches of conference abstracts/ proceedings, systematic reviews and reference lists of included trials. We screened a total of 30,262 records after removal of duplicates. Three review authors (MG, CEW and CB) independently scanned titles and abstracts from the first two searches and the Co-ordinating Editor (Bernd Richter (BR)) and one review author 
(CB) screened titles and abstracts from the third search and fourth search. We did not identify any ongoing trials.

Three review authors (CB, CEW and MG) and the Co-ordinating Editor (BR) assessed eligibility of trials against the inclusion criteria and grouped trials according to similar intervention categories. We identified a total of 41 randomised controlled trials (RCTs) for inclusion in the review (see Characteristics of included studies). The number of trials identified for each intervention category were as follows.
- Changes to the organisation of nutritional care $(\mathrm{N}=13)$

- Changes to the feeding environment $(N=5)$

- Modification of meal profile or pattern $(\mathrm{N}=12)$

- Additional supplementation of meals $(\mathrm{N}=10)$

- Congregate and home meal delivery systems $(\mathrm{N}=1)$

A PRISMA flow-diagram of trial selection is shown in Figure 2. 
Figure 2. Study flow diagram

29,155 records identified through database searching
First search (Inception-March 2013)
Cochrane Library: 3391
MEDLINE: 6049
Embase: 5673
British Nursing Index: 211
CINAHL: 2517
Web of Science: 4525
Scopus: 3236
AMED: 423
Top-up searches (January 2013-September 2016)
Cochrane Library: 428
MEDLINE: 991
Top-up searches (Inception-September 2016)
ClinicalTrials.gov: 1600
WHO ICTRP: 111

1107 additional records identified through other sources (conference abstracts/proceedings, systematic reviews, reference lists)

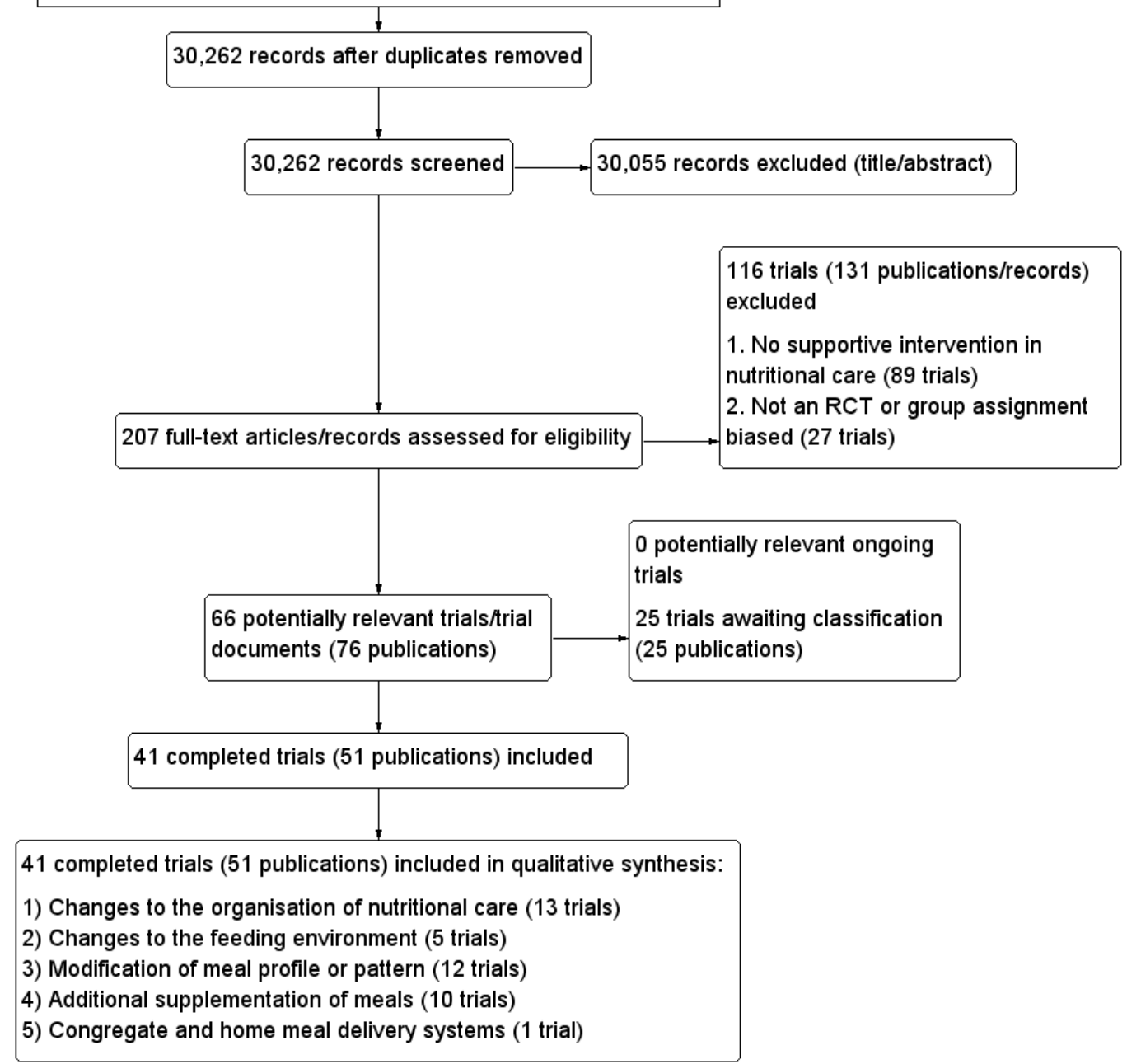

\section{Contact with authors}

Of the 41 included trials, we requested additional information on outcomes of interest and quality from the authors of 31 trials, and obtained it for 15 (Barton 2000; Beck 2002, Bouillanne 2013; Bourdel-Marchasson 2000; Dennis 2005; Duncan 2006; Faxen-Irving 2011; Gaskill 2009; Germain 2006; Hickson 2004; Holyday 2012;
Olofsson 2007; Simmons 2008; Simmons 2010; Smoliner 2008). For six of the 15 trials where the study authors responded, they were unable to provide the data requested, or the data were not usable in a meta-analysis (Barton 2000; Beck 2002; Bourdel-Marchasson 2000; Gaskill 2009; Simmons 2008; Simmons 2010). The authors of the remaining 16 trials did not respond (Castellanos 2009; Chang 2005; Essed 2007; Essed 2009; Hankey 1993; Johansen 2004; Kraft 
2012; Larsson 1990; Lin 2010; Mathey 2001a; Mathey 2001b; Pivi 2011; Potter 2001; Salva 2011; Splett 2003; Van Ort 1995).

\section{Missing data}

Despite the comprehensive search strategies used to identify trials in this review, it is possible that we have missed additional trials (e.g. unpublished trials, those published in obscure places, or those inappropriately indexed in databases).

The largest source of missing data in this review arose from data on outcomes that were measured but reported in such a way that they were unusable for entry into a meta-analysis, because the data were reported as a median and interquartile range or were expressed as $\mathrm{kcal} / \mathrm{kg}$ or the standard deviation (SD ) of change was not reported. The details of the amount of missing data according to intervention group are given in Table 3; Table 4; Table 5; Table 6 and Table 7. We contacted study authors in an attempt to obtain any missing data. The reasons for contacting authors and the outcome of contacts are described in Table 8 and Appendix 11.

Where it was not possible to obtain original data from study authors, we either imputed data, for example, standard deviations, using methods described in the Cochrane Handbook for Systematic Reviews of Interventions (Higgins 2011c), or used formulae for combining groups as outlined in Table 8.

The majority of included trials did not report intention-to-treat analyses.

\section{Dealing with duplicate publications/companion papers}

Six trials included in this review had duplicate or companion publications (Essed 2007; Hickson 2004; Larsson 1990; Lin 2010; Nijs 2006; Potter 2001).

\section{Included studies}

This systematic review identified 41 randomised controlled trials, with a total of 10,681 randomised participants (ranging from 8 (Van Ort 1995) to 4023 (Dennis 2005)). One included trial is awaiting clarification of participant numbers from the study authors (Larsson 1990). This trial had several publications, which stated varying numbers of participants (435 to 501). The primary reference reported data on 435 participants and this is the number that we would use in any meta-analysis (Larsson 1990).

Participants were from a variety of countries including Australia, Brazil, CanadaDenmark, France, Germany, Netherlands, Spain, Sweden, Taiwan, , UK, and USA. Approximately $70 \%$ of participants were female (no information was provided for gender in three trials (Chang 2005; Larsson 1990; Simmons 2008). In those trials that reported ages in the intervention and usual care groups separately $(\mathrm{N}=23)$, the mean age ranged from 62 to 87 years. Where the age of participants was reported for intervention and comparison groups separately, the mean age ranged from 75.2 to $87.3(\mathrm{~N}=11)$ (no data were provided for mean age in three trials (Kretser 2003; Potter 2001; Simmons 2008).

Altogether seven of the 41 included RCTs had a cross-over design (Barton 2000; Castellanos 2009; Essed 2009; Lin 2011; Silver 2008; Simmons 2008; Taylor 2006), 12 a cluster-randomised design (Bourdel-Marchasson 2000; Chang 2005; Gaskill 2009; Leslie 2012; Lin 2010; Lin 2011; Mathey 2001a; Nijs 2006; Salva 2011; Simmons 2008; Smoliner 2008; Splett 2003) and one was a factorial RCT
(Essed 2007). Two trials had both a cluster-randomised and a crossover design (Lin 2011; Simmons 2008). One large trial investigating a normal hospital diet plus oral nutritional supplements versus a normal hospital diet in participants with a recent stroke randomised $38 \%$ participants $(4023 / 10,681)$ of all individuals in the 41 included trials (Dennis 2005).

Interventions were carried out in the hospital setting (described as elderly rehabilitation wards, intermediate care units, geriatric units, acute trauma wards, geriatric acute wards, geriatric orthopaedic wards, medicine for the elderly units and acute medical admissions) $(\mathrm{N}=15)$, residential care homes $(\mathrm{N}=21)$ and free-living or outpatient settings $(\mathrm{N}=5)$ including neurology outpatients, and those enrolled at hospital discharge (see Table 9).

Nutritional status was reported in 27 trials, either because it was assessed at baseline or it was one of the criteria for inclusion in the trial (Beck 2002; Bouillanne 2013; Essed 2007; Essed 2009; FaxenIrving 2011; Gaskill 2009; Germain 2006; Hickson 2004; Holyday 2012; Johansen 2004; Kraft 2012; Kretser 2003; Larsson 1990; Leslie 2012; Lin 2010; Lin 2011; Munk 2014; Nijs 2006; Mathey 2001b; Olofsson 2007; Potter 2001; Remsburg 2001; Salva 2011; Silver 2008; Smoliner 2008; Taylor 2006; Van den Berg 2015). The remaining trials did not assess nutritional status at trial inclusion but we judged them appropriate to be included in this review as the clinical background of trial participants meant that they could be considered to be at risk of malnutrition or the patients were described as frail or vulnerable. Ten of 16 trials used a score from the Mini Nutritional Assessment (MNA) tool of 17 to 23.5 or less than 17 (Beck 2002; Essed 2007; Essed 2009; Holyday 2012; Kretser 2003; Nijs 2006; Olofsson 2007; Salva 2011; Smoliner 2008; Taylor 2006), to indicate risk of malnutrition, one trial used the Subjective Global Assessment score (SGA) (Gaskill 2009), two used the Nutritional Risk Screening 2002 (NRS-2002) tool (Johansen 2004; Munk 2014), eight used only body mass index (BMI) (Faxen-Irving 2011; Hickson 2004; Leslie 2012; Lin 2010; Lin 2011; Mathey 2001b; Remsburg 2001; Silver 2008), four used a combination of indices with variable cut-offs (Bouillanne 2013; Germain 2006; Kraft 2012; Larsson 1990) and one used their own classification scoring system (Potter 2001). The average BMI measurements, in the trials that clearly reported BMI in all participants, ranged from less than $18.5 \mathrm{~kg} / \mathrm{m}^{2}$ (Kretser 2003) to $28.7 \mathrm{~kg} / \mathrm{m}^{2}$ (Nijs 2006)

The most commonly reported outcomes of interest to this review were nutritional intake (predominantly energy and protein), weight and mortality. These were reported in 27, 28 and 18 trials respectively. The three primary outcomes in the review, nutritional intake, health-related quality of life and morbidity and complications, were reported in 27,5 , and 5 trials respectively. Patient satisfaction, hospital admission and costs were reported for a limited number of trials (2,2 and 3 respectively). Six trials reported no usable data for potential combination in a meta-analysis (Beck 2002; Castellanos 2009; Chang 2005; Gaskill 2009; Splett 2003; Van Ort 1995). We contacted the study authors who either were unable to provide the data requested, or failed to respond (see Table 8 and Appendix 11).

The outcomes reported in all intervention groups and those of use in this review, are summarised in Table 7. 


\section{Length of intervention and follow-up}

Length of intervention and follow-up ranged from 'length of hospital stay' to 12 months in the included trials. In one trial, the length of intervention was unclear (Gaskill 2009). In 7 of 38 trials (Brouillette 1991; Dennis 2005; Duncan 2006; Gaskill 2009; Holyday 2012; Johansen 2004; Olofsson 2007) the follow-up period extended beyond the intervention from two weeks to six months.

Further results of the included trials are given in their individual intervention categories (see Appendix 3 for description of interventions).

\section{Changes to the organisation of nutritional care}

We identified 13 trials for this category (Chang 2005; Duncan 2006; Gaskill 2009; Hickson 2004; Holyday 2012; Johansen 2004; Kraft 2012; Lin 2010; Lin 2011; Olofsson 2007; Pivi 2011; Salva 2011; Splett 2003), ( $N=3426,32.4 \%$ of review participants). Participants either had dementia, hip fractures or were from a range of clinical backgrounds, living in residential care homes, hospital or their own homes. Interventions consisted of the use of dietetic assistants (Duncan 2006; Hickson 2004), multidisciplinary team care (Johansen 2004), specialised teaching and training (Chang 2005; Gaskill 2009; Lin 2010; Lin 2011; Pivi 2011; Salva 2011), protocol-driven nutrition care pathways (Holyday 2012; Splett 2003), multicomponent intervention (Olofsson 2007) and monitoring by telemedicine (Kraft 2012). Duration ranged from a few days of hospital stay to 12 months, and follow-up from 28 days to 12 months. We have summarised the outcomes reported, and those usable for this review, Table 4 .

\section{Changes to the feeding environment}

We identified five trials for this category (Brouillette 1991; Mathey 2001a; Nijs 2006; Remsburg 2001; Van Ort 1995), (N = $351,3.3 \%$ of review participants). All trials were conducted in elderly participants living in residential care homes. Interventions consisted of the use of osmotherapy (pre-meal sensory stimulation) (Brouillette 1991), improving mealtime ambience (Mathey 2001a), using family style meals (Nijs 2006), a buffet-style meal service (Remsburg 2001), and a contextual/behavioural intervention (Van Ort 1995). Duration of intervention ranged from 3 weeks to 12 months, and follow-up ranged from 4 weeks to 12 months. We have summarised the outcomes reported, and those usable for this review, in Table 4.

\section{Modification of meal profile or pattern}

We identified 12 trials for this category (Barton 2000; Bouillanne 2013; Castellanos 2009; Essed 2007; Essed 2009; Germain 2006; Leslie 2012; Mathey 2001b; Munk 2014; Silver 2008; Smoliner 2008; Taylor 2006), ( $N=649,6 \%$ of review participants). The trial by Barton 2000 included three groups, two of which were randomised to treatment or control and one other where it was unclear whether there was randomisation. Data have therefore only been included for those participants who were randomised to the treatment and usual care groups $(N=27)$. The trials included people from a range of clinical backgrounds who were in hospital (Barton 2000; Bouillanne 2013; Munk 2014), residential care homes (Castellanos 2009; Essed 2007; Essed 2009; Germain 2006; Leslie 2012; Mathey 2001b; Smoliner 2008; Taylor 2006), and free-living participants in receipt of home-delivered lunch meals (Silver 2008). Interventions consisted of altering portion sizes or fortifying meals, or both (Barton 2000; Castellanos 2009; Leslie 2012; Silver 2008), providing $78 \%$ of daily protein requirements at the lunch time meal, rather than spread evenly throughout the day (Bouillanne 2013), modifying the taste of foods previously identified as preferred (Essed 2007; Essed 2009; Mathey 2001b), modification of the appearance and presentation of pureed foods, thickened beverages, and dietary supplements (Germain 2006), the provision of an a la carte menu of enriched meals (Munk 2014) and altering meal pattern (Taylor 2006). We have summarised the outcomes reported, and those of use in this review, in Table 5.

\section{Additional supplementation of meals}

We identified 10 trials for this category (Beck 2002; BourdelMarchasson 2000; Dennis 2005; Faxen-Irving 2011; Hankey 1993; Larsson 1990; Potter 2001; Simmons 2008; Simmons 2010; Van den Berg 2015) ( $N=6022,56.4 \%$ of review participants). One trial did not state clearly the number of participants as additional publications appeared to include different numbers (Larsson 1990). As stated in the primary reference, 435 participants were therefore included in this review. The trial by Simmons 2008 was a two-phase crossover and cluster-randomised trial where residents were randomised only if they had a low oral food and fluid intake and were responsive to one of two feeding-assistance interventions. This randomised sub-group of intervention and control participants were then crossed over. We used data from the intervention and comparison groups prior to cross-over in this review, as additional participants were added to the trial at the crossover.

One trial (Dennis 2005) included only people who had had a stroke. Other trials included either mixed participants, or did not report diagnoses. The majority of participants were from the hospital setting (Bourdel-Marchasson 2000; Dennis 2005; FaxenIrving 2011; Hankey 1993; Larsson 1990; Potter 2001; Van den Berg 2015), and only 168 were from residential care homes (Beck 2002; Simmons 2008; Simmons 2010). In nine RCTs participants were offered between $400 \mathrm{kcal} / \mathrm{day}$ to $685 \mathrm{kcal} / \mathrm{day}$ in the form of a protein-energy oral nutritional supplement, in addition to usual diet. In the other RCT participants were offered up to $420 \mathrm{kcal}$ extra using $90 \mathrm{~mL}$ of fat emulsion/day (Faxen-Irving 2011). We have summarised the outcomes reported, and those of use in this review, in Table 6.

\section{Congregate and home meal delivery systems}

We identified one trial for this category (Kretser 2003), including 203 free-living participants ( $2 \%$ of review participants). Participants were offered modified home-delivered meals with a daily followup phone call. The outcomes of interest reported in this review included weight, clinical function, Activities of Daily Living score and number of deaths.

\section{Excluded studies}

Of the 182 trials/trial records after eligibility assessment, we excluded 27 trials as they were non-randomised controlled trials or the group assignment was made after randomisation, and 89 trials that did not describe supportive interventions in nutritional care. It was necessary for all four review authors to participate in discussion about the reasons for exclusion of trials from intervention category four, 'additional supplementation of meals'. Trials were excluded in this group for the following reasons. 
- Participants were not from an institutionalised setting therefore it was considered that they would have been given individualised advice on taking oral nutritional supplements.

- No clear organisational component to the intervention was described (for example when supplements were given without a clear description of delivery (i.e. administered at the same time as medication, or in place of usual morning/afternoon tea), or frequency of delivery).

- Trials with multi component interventions where it was not possible to extract data relating to the specific effect of nutritional intervention.

Twenty-four trials are awaiting assessment.
See Characteristics of excluded studies.

\section{Risk of bias in included studies}

The judgements made about risk of bias for individual trials are detailed in the 'risk of bias' section (Characteristics of included studies). A 'Risk of bias summary', and 'Risk of bias graph' are shown in Figure 3 and Figure 4. We judged the majority of criteria used in the assessment of risk of bias as unclear, indicating insufficient information to permit a full assessment of the risk of bias. The exceptions were attrition bias and reporting bias, where we judged the majority of trials ( $61 \%$ and $76 \%$ respectively) as being at low risk of bias (Figure 4). 
Figure 3. Risk of bias summary: review authors' judgements about each risk of bias item for each included trial.

\begin{tabular}{|c|c|c|c|c|c|c|c|}
\hline & 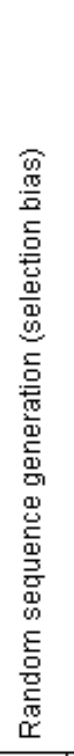 & 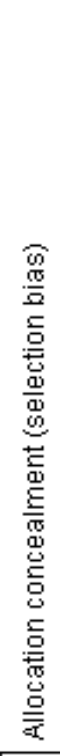 & 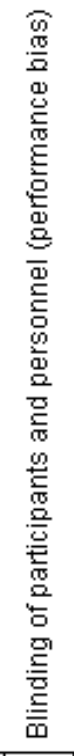 & 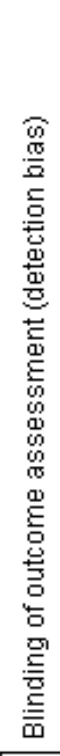 & 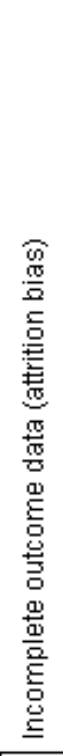 & 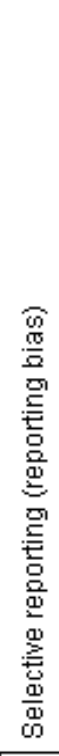 & 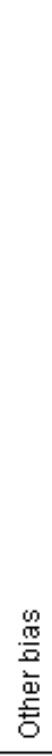 \\
\hline Barton 2000 & $?$ & $?$ & + & $?$ & $?$ & + & $?$ \\
\hline Beck 2002 & $?$ & $?$ & $?$ & $?$ & $?$ & + & $?$ \\
\hline Bouillanne 2013 & $?$ & $?$ & $?$ & $?$ & $\odot$ & $\odot$ & $\odot$ \\
\hline Bourdel-Marchasson 2000 & $?$ & $?$ & $?$ & $?$ & $?$ & $\odot$ & - \\
\hline Brouillette 1991 & $?$ & $?$ & 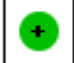 & + & + & + & + \\
\hline Castellanos 2009 & $?$ & $?$ & $?$ & $?$ & $\odot$ & - & $?$ \\
\hline Chang 2005 & $\odot$ & $?$ & $?$ & $?$ & - & + & $?$ \\
\hline Dennis 2005 & + & + & $?$ & $\odot$ & + & + & $?$ \\
\hline Duncan 2006 & $?$ & + & $?$ & + & $\odot$ & + & 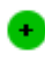 \\
\hline Essed 2007 & $?$ & $?$ & - & $?$ & $\odot$ & + & $\odot$ \\
\hline Essed 2009 & $?$ & $?$ & $?$ & $?$ & + & $?$ & $?$ \\
\hline Faxen-Irving 2011 & $?$ & - & - & $?$ & $\odot$ & + & - \\
\hline Gaskill 2009 & $?$ & $?$ & $?$ & $?$ & $?$ & + & - \\
\hline Germain 2006 & $?$ & + & $?$ & $?$ & + & + & $\odot$ \\
\hline Hankey 1993 & $?$ & $?$ & $?$ & $?$ & + & + & $?$ \\
\hline Hickson 2004 & $\odot$ & + & $?$ & $?$ & $\odot$ & - & $\odot$ \\
\hline Holyday 2012 & + & $?$ & - & $\theta$ & $\odot$ & + & + \\
\hline Johansen 2004 & $\odot$ & $?$ & $?$ & $?$ & $?$ & + & $\odot$ \\
\hline Kraft 2012 & $?$ & $?$ & $?$ & $?$ & $\theta$ & $\odot$ & $\odot$ \\
\hline Kretser 2003 & -1 & $?$ & $?$ & $?$ & $?$ & + & $?$ \\
\hline
\end{tabular}


Figure 3. (Continued)

\begin{tabular}{|c|c|c|c|c|c|c|c|}
\hline Kretser 2003 & $\odot$ & $?$ & $?$ & $?$ & $?$ & $\oplus$ & $?$ \\
\hline Larsson 1990 & $?$ & $?$ & $?$ & $?$ & $?$ & 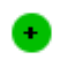 & $\odot$ \\
\hline Leslie 2012 & $?$ & + & $?$ & $?$ & + & 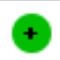 & $\odot$ \\
\hline Lin 2010 & $?$ & $?$ & $?$ & + & $?$ & + & $\Theta$ \\
\hline Lin 2011 & $?$ & $?$ & $?$ & $\odot$ & $?$ & $\oplus$ & $\odot$ \\
\hline Mathey 2001a & $?$ & $?$ & $?$ & $?$ & $\odot$ & 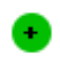 & $\odot$ \\
\hline Mathey $2001 \mathrm{~b}$ & $?$ & $?$ & $?$ & $?$ & $\odot$ & $\odot$ & $\oplus$ \\
\hline Munk 2014 & + & + & $\odot$ & $\odot$ & + & + & + \\
\hline Nijs 2006 & $\odot$ & $\odot$ & $?$ & $?$ & $\odot$ & $\odot$ & $\odot$ \\
\hline Olofsson 2007 & $?$ & $\odot$ & $\odot$ & $\odot$ & $\odot$ & $\odot$ & $?$ \\
\hline Pivi 2011 & $?$ & $?$ & $?$ & $?$ & $\odot$ & $\oplus$ & $?$ \\
\hline Potter 2001 & $?$ & $?$ & $\odot$ & $?$ & $?$ & $\odot$ & $\odot$ \\
\hline Remsburg 2001 & $?$ & $?$ & $?$ & $?$ & $\odot$ & $?$ & $\odot$ \\
\hline Salva 2011 & $?$ & $?$ & $?$ & $?$ & $\odot$ & $\oplus$ & $\odot$ \\
\hline Silver 2008 & $?$ & $?$ & $?$ & $?$ & $?$ & + & $?$ \\
\hline Simmons 2008 & $\odot$ & $?$ & $\odot$ & $\odot$ & $?$ & $?$ & $\odot$ \\
\hline Simmons 2010 & $?$ & $?$ & $?$ & $?$ & + & $?$ & $?$ \\
\hline Smoliner 2008 & $?$ & $?$ & $?$ & $?$ & 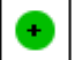 & $?$ & $\odot$ \\
\hline Splett 2003 & + & $?$ & $?$ & $?$ & + & + & $\odot$ \\
\hline Taylor 2006 & $?$ & $?$ & $?$ & $?$ & $?$ & $?$ & $?$ \\
\hline Van den Berg 2015 & $\odot$ & + & $\odot$ & $\odot$ & $\odot$ & + & $\oplus$ \\
\hline Van ort 1995 & $?$ & $?$ & $?$ & $?$ & $?$ & $\odot$ & $?$ \\
\hline
\end{tabular}


Figure 4. Risk of bias graph: review authors' judgements about each risk of bias item presented as percentages across all included trials.

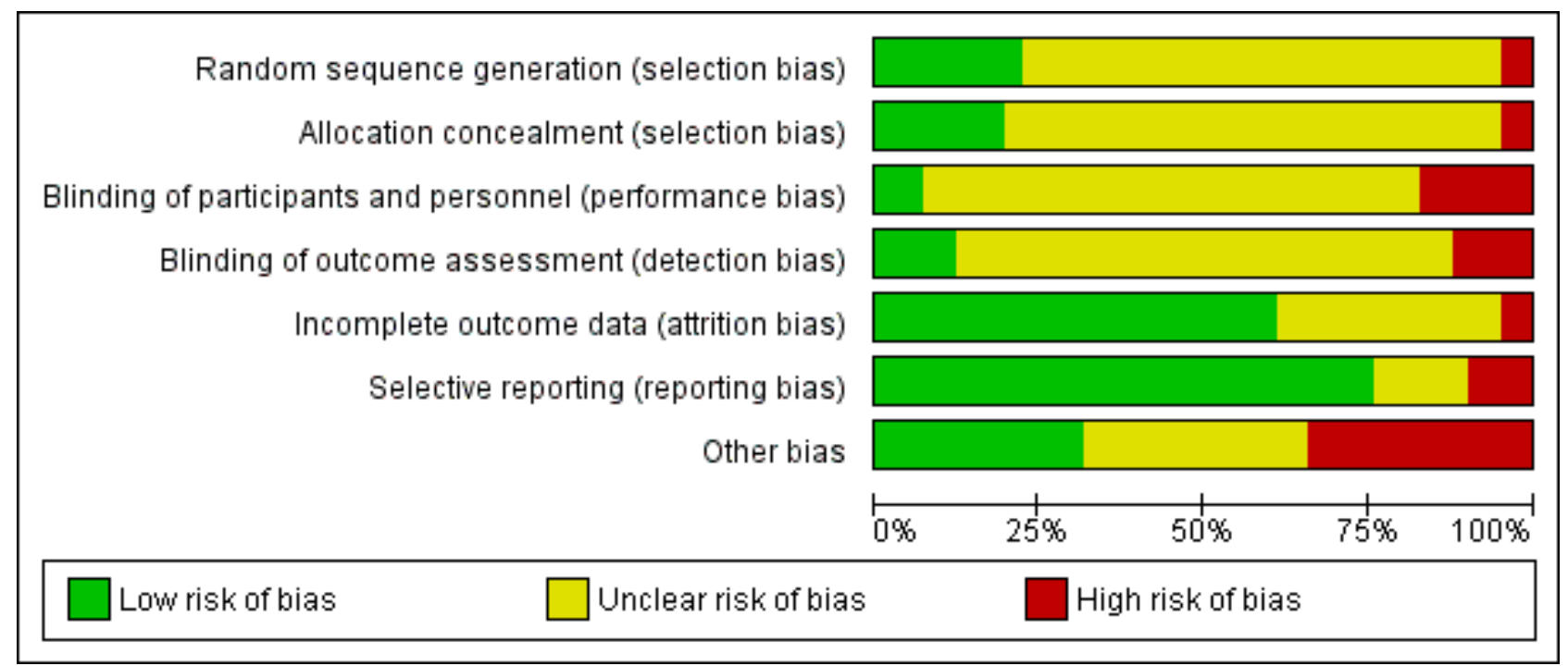

\section{Allocation}

\section{Generation of sequence}

We assessed nine of 41 trials (Chang 2005; Dennis 2005; Hickson 2004; Holyday 2012; Johansen 2004; Munk 2014; Simmons 2008; Splett 2003; Van den Berg 2015), as being at low risk of bias for the method of random sequence generation. Two of these trials used the toss of a coin as a method of randomisation (Chang 2005; Simmons 2008), one used a sequence generated by a member of staff not involved in the trial (Munk 2014) and another used a random number table (Splett 2003). The other trials in this group used computer-generated randomisation methods.

Two of 41 trials ( Kretser 2003; Nijs 2006) used inadequate methods of randomisation and we consequently gave them a high risk of bias. In another trial (Kretser 2003) the authors stated "randomised treatment assignment was followed with a few exceptions". When the participants were randomised to receive the new meals on wheels and refused, they were automatically placed on the traditional meals on wheels model. We therefore considered that allocation was made by preference of the participant. In the trial by Nijs 2006 the investigators described a non-random component in the sequence generation process, based on the name of the ward. This was therefore given a high risk of bias score.

One trial did not detail whether the third intervention group was randomised, and subsequently received an unclear risk of bias (Barton 2000). The remaining trials in the review provided insufficient information about the sequence generation process to permit judgement of low or high risk of bias. We therefore categorised them as unclear risk of bias.

\section{Allocation concealment}

We assessed eight of 41 trials (Dennis 2005; Duncan 2006; Germain 2006; Hickson 2004; Leslie 2012; Munk 2014; Olofsson 2007; Van den Berg 2015), as being at low risk of bias for allocation concealment , as they used sequentially numbered or opaque sealed envelopes opened by a member of staff not involved in the trial, or allocation was made by a statistician having no other contact with the participants. The trial by Faxen-Irving 2011 was considered to be at a high risk of allocation concealment, as they used sealed envelopes without describing the appropriate safeguards, for example, not sequentially numbered, or opaque. This suggested that participants, or investigators enrolling participants, could predict assignments, and thus introduce selection bias. Another trial used no concealment and therefore we judged it to be at a high risk of bias (Nijs 2006). The remaining trials included in the review we categorised as unclear risk of bias, as they provided insufficient information to permit a full assessment of the risk of bias.

\section{Blinding}

\section{Blinding of participants and personnel (performance bias)}

We judged three of 41 trials (Barton 2000; Brouillette 1991; Potter 2001) to be at a low risk of bias, as the trial participants were blind to group allocation or to what treatment they were receiving. We also judged that blinding was unlikely to have been broken throughout the trials. To give examples, in the trial by Barton 2000 the participants and staff were blinded to which menu they were following. In the trial by Brouillette 1991, the research assistant was unaware of group assignment. We awarded Potter 2001 a low risk of bias score, as researchers who knew the randomisation codes were not involved in outcome data collection or data entry.

We judged seven of 41 trials (Essed 2007; Faxen-Irving 2011; Holyday 2012; Munk 2014; Olofsson 2007; Simmons 2008; Van den Berg 2015) to be at high risk of bias, predominantly due to a lack of blinding of key trial personnel. In the trial by Essed 2007 there was incomplete blinding, as participants were blinded but the research personnel were not. In the trial by Faxen-Irving 2011, study nurses opened sealed envelopes, therefore would have been aware of group allocation. In the trial by Holyday 2012, the authors stated it was not possible to blind the clinical dietitian to group allocation. We therefore judged that the outcome was likely to be influenced by a lack of blinding of key trial personnel. Additionally, the trial by Olofsson 2007 stated that staff on the usual care ward were aware of a programme being implemented on another ward in the hospital. It was therefore judged that outcome assessment was likely to be 
influenced by lack of blinding to these key trial personnel. The remaining trials in the review we categorised as unclear risk of bias, as insufficient information was provided to permit judgement.

\section{Blinding of outcome assessment (detection bias)}

We judged five of 41 trials (Brouillette 1991; Duncan 2006; Lin 2010; Lin 2011; Olofsson 2007) to be at low risk of bias. Researchers assessing outcomes were unaware of treatment allocation; therefore we judged that the blinding was unlikely to have been broken. We judged five of 41 trials (Dennis 2005; Holyday 2012; Munk 2014; Simmons 2008; Van den Berg 2015) as at high risk of bias, as outcome assessment was not blinded, and the outcome measurement was likely to be influenced by the lack of blinding. One trial stated, "as the outcomes are primarily objective measures, they are mostly not open to the influence of bias" (Holyday 2012). Additionally, the trial by Dennis 2005 stated "follow up was masked to treatment allocation except when patients or carers inadvertently divulged it to an interviewer, which was usually, but not systematically recorded". In the trial by Simmons 2008 outcomes were not assessed blinded to treatment and the outcomes were judged to be susceptible to detection bias. In the trial by Van Ort 1995, the research staff who observed videotapes were unaware of the trial hypothesis, but were aware of group allocation. We gave this trial, and the remaining 28 trials, an unclear risk of bias, as insufficient information was provided to permit judgement of the risk of bias.

\section{Incomplete outcome data}

The numbers of participants excluded from trials, along with reasons, were fully reported in 25 out of 41 trials and we judged these to have a low risk of bias. The number of participant exclusions ranged from $0 \%$ to $81 \%$. The trial by Chang 2005 we judged to be at high risk of bias, because data were presented on only 20 of the 36 participants, without explanation. We judged another trial as high risk due to the high attrition rate in the intervention group (Kraft 2012). Here, eight participants out of 13 in the intervention group withdrew, and three out of 13 in the usual care group withdrew.

We included a total of 14 trials in the unclear risk of bias category. Three trials did not report exclusions (Barton 2000; Beck 2002; Simmons 2008). One of these is awaiting clarification from the trial author (Beck 2002), and another only reported participant exclusions in one of the intervention groups (Barton 2000). In a further three trials, the numbers of exclusions were unclear (Bourdel-Marchasson 2000; Gaskill 2009; Larsson 1990). Six trials only reported a total number finishing the trial, rather than a breakdown for the intervention and usual care groups separately (Johansen 2004; Kretser 2003; Lin 2010; Silver 2008; Taylor 2006; Van Ort 1995). Each of these trials stated why participants dropped out, however it was unclear which group they were allocated to. Simmons 2008 reported dropouts from each group, however only described mortality as the primary reason (58\%). One trial did not describe attrition (Lin 2011), and another trial reported outcome in relation to $\mathrm{BMI}$ and triceps skinfold thickness (TSF), but not BMI and TSF alone (Potter 2001).

\section{Selective reporting}

Thirty-one of the 41 trials reported all outcomes as stated in the trial methodology, and we therefore judged them to be at low risk of bias. We categorised four trials as high risk of bias (Castellanos
2009; Hickson 2004; Potter 2001; Van Ort 1995). In the trial by Potter 2001, one or more outcomes of interest to the review were described as collected but were incompletely reported. In another trial, results for the whole group were not reported according to the initial randomisation (Castellanos 2009). In the trial by Hickson 2004, no data were reported on: use of service questionnaires, referral rate to therapists, readmission within six months, laxative use, pressure sores and economic analysis. In the trial by Van Ort 1995, outcomes were described in the methodology, however no quantitative data were reported. We categorised the remaining six trials as unclear risk of bias (Essed 2009; Remsburg 2001; Simmons 2008; Simmons 2010; Smoliner 2008; Taylor 2006), as insufficient information was provided in order to make a judgement on risk of bias.

\section{Other potential sources of bias}

We judged 13 of the 41 trials as low risk of bias, as intervention and usual care groups were comparable at baseline (Bouillanne 2013; Brouillette 1991; Duncan 2006; Essed 2007; Germain 2006; Hickson 2004; Holyday 2012; Johansen 2004; Kraft 2012; Mathey 2001b; Munk 2014; Remsburg 2001; Van den Berg 2015). In Hickson 2004, there were significantly more women in the intervention compared with the usual care group, but otherwise groups were comparable. Three parallel RCTs were judged at high risk of bias (Faxen-Irving 2011; Larsson 1990; Potter 2001). Faxen-Irving 2011 provided data only from those who completed the trial, potentially missing valuable data for those who dropped out. In the trial by Larsson 1990, there were significant differences between groups at baseline. TSF and weight index in men, and mid-arm circumference (MAC) in women were significantly lower in the intervention group than the control. The intervention group also had a significantly poorer mental condition as assessed using the modified Norton score on admission. In the trial by Potter 2001, only half of those in the 'well nourished' group were randomised, therefore bias was likely to have occurred. We categorised 14 trials as unclear risk of bias, as there was insufficient information to assess whether an important risk of bias existed.

We considered the following risk of bias criteria for the 12 clusterRCTs (Bourdel-Marchasson 2000; Chang 2005; Gaskill 2009; Leslie 2012; Lin 2010; Lin 2011; Mathey 2001a; Nijs 2006; Salva 2011; Simmons 2008; Smoliner 2008; Splett 2003): (a) recruitment bias, (b) baseline imbalance, (c) loss of clusters, (d) incorrect analysis, and (e) comparability with individually randomised trials or different types of clusters as described in the Cochrane Handbook for Systematic Reviews of Interventions (Higgins 2011c). If any of the aforementioned criteria applied, we assigned a high risk of 'other bias'. Consequently, all included cluster RCTs had a high risk of bias. In the trial by Chang 2005 it was unclear whether randomisation occurred at the unit level (more probable) or the individual level. We therefore judged this trial to be an unclear risk of other bias.

\section{Effects of interventions}

See: Summary of findings for the main comparison Supportive interventions for enhancing dietary intake versus comparators in malnourished or nutritionally at-risk adults

We could not recalculate data taking into account the design effect for the 12 cluster RCTs (Bourdel-Marchasson 2000; Chang 2005; Gaskill 2009; Leslie 2012; Lin 2010; Lin 2011; Mathey 
2001a; Nijs 2006; Salva 2011; Simmons 2008; Smoliner 2008; Splett 2003) because we did not have reliable information about intracluster correlation coefficients for our substantial heterogeneous populations in the included trials. Therefore, we did not establish meta-analyses by using both parallel and cluster RCTs but excluded the cluster RCTs from all meta-analyses. Also, crossover trials did not contribute to the effect estimates established by meta-analyses.

\section{Overview of all trials combined}

\section{Primary Outcomes}

\section{Nutritional intake}

Data on this outcome were reported in 27 of 41 trials (Barton 2000; Beck 2002; Bouillanne 2013; Bourdel-Marchasson 2000; Brouillette 1991; Castellanos 2009; Chang 2005; Duncan 2006; Essed 2007; Essed 2009; Faxen-Irving 2011; Germain 2006; Hankey 1993; Hickson 2004; Johansen 2004; Leslie 2012; Lin 2010; Mathey 2001a; Mathey 2001b; Munk 2014; Nijs 2006; Potter 2001; Silver 2008; Simmons 2008; Simmons 2010; Taylor 2006; Van den Berg 2015).

The trials reporting on change in energy intake were in participants from a range of clinical backgrounds and healthcare settings and there were differences between trials in how energy intake was assessed (from observations of amounts eaten to detailed weighing and analysis). The majority of trials found no marked difference in energy intake between groups. One trial of assistance at mealtimes in hospitalised patients with hip fracture (Duncan 2006) reported a greater energy intake in the intervention group than in the usual care group (1105 kcal (SD 361) versus 759 (SD 399), $P<0.001$ ) and a trial of a multidisciplinary team intervention in hospitalised patients (Johansen 2004) reported a higher intake in the intervention group than in the control group (Table 10). Two trials of fortification of meals (Barton 2000; Silver 2008) reported greater energy intakes in participants receiving the fortification than those receiving usual care (Table 15) and one trial of modifications to the appearance and presentation of foods to individuals with dysphagia (Germain 2006) reported a greater energy intake in the participants receiving the intervention (Table 15). Two of 10 trials of supplementation of meals with oral nutritional supplements (Hankey 1993; Van den Berg 2015) reported a higher energy intake in groups receiving the supplement, however the between-group differences were not reported (Table 19).

\section{Health-related quality of life and patient satisfaction}

Data on health-related quality of life were reported in five of 41 trials (Dennis 2005; Johansen 2004; Mathey 2001a; Nijs 2006; Smoliner 2008). Data were collected using different quality-of-life instruments; two trials used the Short Form-36 (SF-36) (Johansen 2004; Smoliner 2008), one trial used the Dutch quality of life of somatic nursing home residents questionnaire (Nijs 2006), one used the European Quality of Life Scale (EuroQOL-5D or EQ-5D) (Dennis 2005) and the final trial (Mathey 2001a) used the Sickness Impact Profile (SIP) and Philadelphia Geriatric Center Morale Scale (PGCMS, 17 items). The trials reporting on healthrelated quality of life included participants from a wide range of different clinical backgrounds. No marked differences between groups were found in four trials (Dennis 2005; Johansen 2004; Mathey 2001a; Smoliner 2008) (Table 11; Table 16; Table 23), the overall quality of evidence was low and two trials were cluster- randomised trials and therefore at high risk of bias (Mathey 2001a; Smoliner 2008). Nijs 2006 assessed health-related quality of life using a validated Dutch questionnaire (Van Campen 1998). This questionnaire consists of five sub-scales, each representing a quality-of-life dimension: sensory functioning (focusing on pain); physical functioning (perceived performance and self care); psychosocial functioning (depression or loneliness); perceived autonomy (freedom of movement); and perceived safety (feeling at home in the institution). The number of statements in the five subscales is not equal. The questionnaire consists of 50 statements, scored on a dichotomous scale (yes or no). Each sub-scale and the total questionnaire is computed to achieve a score from 0 to 100. A high score represents a high quality of life. The results were presented as difference in changes in overall quality of life between the groups and were reported as statistically significant (6.1 units, 95\% confidence interval $(\mathrm{Cl}) 2.1$ to 10.3$)$. The intervention group remained stable ( 0.4 units, $95 \% \mathrm{Cl} 1.8$ to 2.5 ), whereas the usual care group declined ( -0.5 units, $95 \% \mathrm{Cl}-9.4$ to 0.6$)$, although the overall changes were small and it is unclear if the observed differences were likely to be noticeable to participants (Table 16). Moreover, this trial was at high risk of bias. Therefore, all reported outcome measures of this trial must be interpreted with caution.

Data on patient satisfaction were reported in two trials (Duncan 2006; Salva 2011). Duncan 2006 assessed patient satisfaction using an unvalidated questionnaire with 10 questions about aspects of meals, diet and feeding. Participants answered yes or no, where yes $=1$, no $=-1$ and NA $=0$. Those participants who had received the support of the dietetic assistants showed greater satisfaction, with a median score of 6.5 (interquartile range (IQR) 2) compared to 3 (IQR 4) for participants receiving usual care $(P<0.0001)$ (Table 11$)$. In the trial by Salva 2011 satisfaction of participants and their families was assessed by an unvalidated questionnaire which asked about the use of and perceived usefulness of five aspects of the overall programme. Families and carers were asked to indicate whether they had used the service and whether they had found it very useful, useful or not very useful. Information cards were used by $94.5 \%$ of families and rated the service as very useful $(26 \%)$ or useful $(67 \%)$. The nutrition course was used by $66 \%$ of families and rated as very useful (24\%) and useful (65\%). Weight curves were sent to $88 \%$ of families and rated as very useful (13\%) and useful $(78 \%)$. Information sessions were attended by $75 \%$ of families and rated as very useful (32\%) and useful (61.5\%). The hot line was used by $33 \%$ of families and rated as very useful (17\%) and useful (51\%).

\section{Morbidity/complications}

Data on this outcome were reported in seven of 41 trials (Bouillanne 2013; Bourdel-Marchasson 2000; Dennis 2005; Duncan 2006; Hickson 2004; Johansen 2004; Olofsson 2007). Complications were reported as either the number of participants experiencing any complication (Bouillanne 2013; Dennis 2005; Duncan 2006; Johansen 2004; Olofsson 2007), number of participants with pressure ulcers (Bourdel-Marchasson 2000; Dennis 2005) or the number of participants needing oral antibiotics (Hickson 2004). Trials were in participants from different clinical backgrounds, in different healthcare settings and receiving interventions that aimed to be supportive of improved nutritional intake, and varied widely. There were no marked differences in complication rates between groups reported in any trial (Table 11).

Meta-analysis of trials reporting number of participants experiencing any complication showed considerable inconsistency 
$\left(1^{2}=91 \%\right)$. Risk ratios ranged between 0.59 indicating benefit for supportive interventions, to 1.42 indicating benefit of control interventions ( 5 trials; 4015 participants; very low-quality evidence; Analysis 1.1).

\section{Secondary Outcomes}

\section{Nutritional status}

\section{Weight change}

Data on this outcome were reported in 28 of 41 trials (Beck 2002; Bouillanne 2013; Chang 2005; Duncan 2006; Essed 2007; FaxenIrving 2011; Germain 2006; Hankey 1993; Hickson 2004; Holyday 2012; Johansen 2004; Kraft 2012; Kretser 2003; Larsson 1990; Leslie 2012; Lin 2010; Mathey 2001a; Mathey 2001b; Munk 2014; Nijs 2006; Olofsson 2007; Pivi 2011; Potter 2001; Remsburg 2001; Salva 2011; Simmons 2008; Simmons 2010; Smoliner 2008). Trials were in participants from different clinical backgrounds, in different healthcare settings and receiving interventions which, although aiming to support improved nutritional intake, varied from one another in the nature of the intervention.
Meta-analysis across 17 trials with adequate data on weight change revealed an overall improvement in weight in favour of supportive interventions versus control: mean difference (MD) $0.6 \mathrm{~kg}(95 \%$ $\mathrm{Cl} 0.21$ to 1.02$) ; \mathrm{P}=0.003 ; 2024$ participants; moderate-quality evidence; Analysis 1.2. However, heterogeneity was moderate $\left(\mathrm{I}^{2}=\right.$ 51\%). We excluded the trial by Pivi 2011 from this meta-analysis because missing SDs for weight change could not be reliably imputed. Trial authors reported a significant difference between intervention groups using a $P$ value $<0.001$. Using a $P$ value of 0.0005 for imputation of SDs resulted in an SD of 3.3. Using these data did not substantially alter the effect estimate. Some other trials showed bias from different sources, however, exclusion of these trials did not substantially change the overall effect estimate. Also, elimination of any subtype of supportive intervention did not change the overall effect estimate in a substantial way. The body of evidence for this outcome consisted mainly of trials on change to the organisation of nutritional care ( 6 trials). However, the interaction test for subgroup differences was significant indicating the need to further investigate the various types of supportive interventions in future trials (Figure 5).

Figure 5. Forest plot of comparison: 1 Supportive interventions for enhancing dietary intake versus comparators, outcome: 1.2 Nutritional status (weight change) (kg)

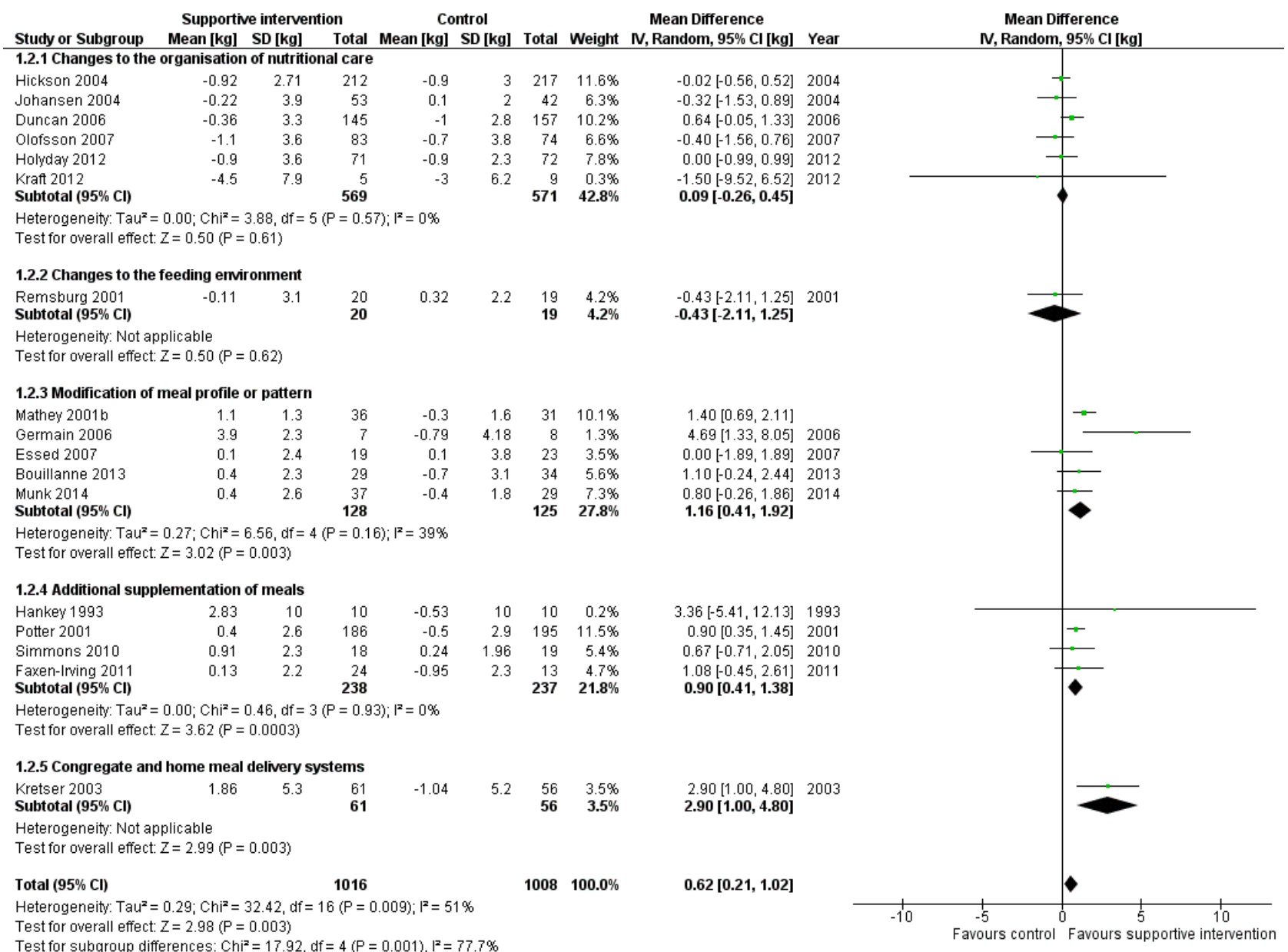

\section{Change in BMI}

Data on change in BMI were reported in 12 of 41 trials (FaxenIrving 2011; Germain 2006; Hickson 2004; Kraft 2012; Leslie 2012;

Lin 2010; Lin 2011; Olofsson 2007; Pivi 2011; Salva 2011; Simmons 2008; Smoliner 2008). Trials were in participants from different clinical backgrounds, in different healthcare settings and receiving interventions that aimed to support improved nutritional intake 
but varied from one another. The majority of trials reported no marked difference in BMI between groups. In the trial by Pivi 2011 participants receiving specialist training experienced an increase in BMI $\left(1.2 \mathrm{~kg} / \mathrm{m}^{2}\right.$ (SD 1)) and participants in the usual care group experienced a reduction in BMI $\left(-2.2 \mathrm{~kg} / \mathrm{m}^{2}\right.$ (SD 1)). However, the between-group difference and statistical tests were not reported. The trial by Germain 2006, which examined the effects of modifications to the presentation of meals to participants with dysphagia, and in the trial by Leslie 2012 of food fortification in residential care homes, the intervention group had a greater gain in BMI than the usual care group (Table 17). However, between-group differences with statistical tests were not reported. In the trial by Faxen-Irving 2011 BMI was reported according to group at the end of the intervention and there was no marked difference between groups, change from baseline and between-group differences were not reported. In the trial by Simmons 2008 the intervention group gained $0.7 \mathrm{~kg} / \mathrm{m}^{2}$ more than the usual care group $(P<0.009)$ (Table 24).

\section{Change in TSF}

Data on this outcome were reported in five of 41 trials (Duncan 2006; Hankey 1993; Hickson 2004; Larsson 1990; Pivi 2011). Trials were in participants receiving assistance during mealtimes (Duncan 2006; Hickson 2004), specialist training (Pivi 2011) and supplementation with oral nutritional supplement (Hankey 1993; Larsson 1990) in different healthcare settings. There were no marked differences in TSF reported between groups in the trials by Duncan 2006, Hickson 2004 and Pivi 2011. In the trials by Hankey 1993 and Pivi 2011 data were presented in figures with minimal description in the text. In the trial by Hankey 1993 the intervention group was described as experiencing a smaller decrease in TSF than the usual care group $(6.6 \%$ versus $15.8 \%)$. In the trial by Larsson 1990 TSF decreased over the 26 weeks of follow-up in both groups with the greatest decrease occurring in the usual care group.

\section{Change in MAC}

Data on this outcome were reported in eight of 41 trials (Duncan 2006; Hankey 1993; Hickson 2004; Larsson 1990; Leslie 2012; Nijs 2006; Pivi 2011; Potter 2001). Trials were in participants from different clinical backgrounds, in different healthcare settings and receiving interventions which aimed to support improved nutritional intake but varied from one another. Three trials reported no marked difference in MAC between groups (Hickson 2004; Nijs 2006; Potter 2001). In the trial by Duncan 2006, the group that received assistance with eating had a smaller reduction in MAC of $-0.9 \mathrm{~cm}$ (SD 2.2) compared with the group that received usual care, -1.3 (SD 1.5) $(P=0.002)$. One trial evaluating the impact of specialist training in free-living individuals (Pivi 2011) reported improvements in MAC in the intervention group of $1.9 \mathrm{~cm}$ (SD 2) compared with a reduction of $-0.4 \mathrm{~cm}$ (SD 0.5) in the group receiving usual care. In the trial by Leslie 2012 of food fortification in residential care homes, participants in the intervention group had a greater improvement in MUAC than those in the control group but the between-group differences and statistical tests were not reported (Table 20) In the trial by Hankey 1993, the data were unavailable from the original trial report but we obtained them from a systematic review by Milne 2009. We read the figures for change from a graph, and we assumed the SD of change to be $10 \mathrm{~cm}$ for each group. MAC was described as improving in the intervention group $(P<0.05)$ but remaining unchanged in the usual care group. The changes were small and no between-group differences were reported (Table 24). In the trial by Larsson 1990 the data are presented in a figure with some description in the text, participants who were well nourished at the start of the trial and received supplementation of meals experienced less decrease in MAC at 26 weeks $(P<0.05)$ than those receiving usual care. In participants who were malnourished at the start of the trial both groups experienced a decrease in MAC at 26 weeks.

\section{Clinical function}

Data on this outcome were reported in nine of 41 trials (Bouillanne 2013; Duncan 2006; Faxen-Irving 2011; Hickson 2004; Kretser 2003; Munk 2014; Potter 2001; Salva 2011; Smoliner 2008). Trials were in participants from a variety of different clinical backgrounds, in different healthcare settings and were assessed using a variety of methods including handgrip strength, Barthel score, Activities of Daily Living (ADL), instrumental ADL (iADL) and peak flow.

Three trials assessed functional recovery using the Barthel score (Hickson 2004; Smoliner 2008; Potter 2001). The Barthel index consists of 10 items that measure a person's daily functioning, specifically the activities of daily living and mobility (Mahoney 1965). The items include feeding, moving from wheelchair to bed and return, grooming, transferring to and from a toilet, bathing, walking on level surface, going up and down stairs, dressing, continence of bowels and bladder. The items are weighted according to a scheme developed by the authors. The person receives a score based on whether they have received help while doing the task. The scores for each of the items are summed to create a total score. The higher the score the more 'independent' the person. Independence means that the person needs no assistance with any part of the task. There were no marked differences between groups in any trial. In the trial by Potter 2001 there was no marked difference in numbers achieving functional recovery assessed using the Barthel index in the group receiving supplementation compared with the usual care group (102/149 intervention versus $100 / 157$ control, $\mathrm{P}=0.38$ ). However, more participants classified as severely undernourished experienced an improvement in their Barthel scores on supplementation compared with those that received usual care $(17 / 25$ intervention versus $11 / 28$ control, $\mathrm{P}<0.04$ ).

Four trials assessed clinical function using the $A D L$ and $i A D L$ scores (Bouillanne 2013; Faxen-Irving 2011; Kretser 2003; Salva 2011). Two main types of abilities are measured by these functional assessment scales. Basic ADL consist of activities that are performed daily, habitually and universally, such as dressing, bathing, and eating. In contrast, iADL requires organisation and planning, and includes such tasks as shopping, using transportation, preparing meals, handling finances, keeping the house, and using a telephone. The scores range from 0 to 100 and amount of functional impairment is then rated as "none to mild" (0 to 33), "moderate" (34 to 66), or "severe" (>66). All trials reported no marked differences in ADL between the intervention and usual care groups. One trial used the iADL (Kretser 2003) to measure clinical function. There was a greater decline in $\mathrm{ADL}$ in those receiving traditional meals on wheels compared with those receiving modified meals on wheels at six months $(P=0.0494)$.

Five trials assessed clinical function using handgrip strength (Bouillanne 2013; Duncan 2006; Hickson 2004; Munk 2014; Smoliner 2008), and there were no marked differences in any trial between 
the groups receiving the intervention and those receiving usual care (Table 13; Table 21).

In the trial by Smoliner 2008 clinical function was also measured using peak flow. Peak expiratory flow is the maximum flow generated during expiration performed with maximal force and started after a full inspiration. A decrease in peak flow rates indicates a deterioration in clinical function and vice versa. The peak flow in the intervention group increased from baseline to follow-up (12 weeks) (mean $152 \mathrm{~mL} / \mathrm{min}$ (SD 105) to $186 \mathrm{~mL} / \mathrm{min}$ (SD 140) whereas the usual care showed a decline $(151 \mathrm{~mL} / \mathrm{min}$ (SD90) to $150 \mathrm{~mL} / \mathrm{min}$ (SD 67). The between-group difference was statistically significant $(P=0.039)$.

\section{Hospitalisation and institutionalisation}

Data on length of hospital stay were reported in 10 of 41 trials (Dennis 2005; Duncan 2006; Faxen-Irving 2011; Hickson 2004; Holyday 2012; Johansen 2004; Munk 2014; Olofsson 2007; Potter 2001; Van den Berg 2015). The trials were either of changes to the organisation of nutritional care (Duncan 2006; Hickson 2004; Holyday 2012; Johansen 2004; Olofsson 2007 ), fortification of meals in hospital (Munk 2014) or of supplementation of meals with oral nutritional supplements (Dennis 2005; Faxen-Irving 2011; Potter 2001: Van den Berg 2015 ). Nine trials reported no marked difference in length of hospital stay between groups (Dennis 2005; Duncan 2006; Faxen-Irving 2011; Hickson 2004; Holyday 2012; Johansen 2004; Munk 2014; Potter 2001; Van den Berg 2015). In the trial by Olofsson 2007 groups receiving a multidisciplinary team intervention had a shorter mean length of hospital stay (27.4 days (SD 15.9)) than groups receiving usual care (39.8 days (SD 41.9)) ( $P$ $<0.05$ ) (Table 14).

Meta-analysis across five trials with adequate data on length of hospital stay showed a MD between intervention and comparator groups of -0.5 days ( $95 \% \mathrm{Cl}-2.6$ to 1.6$) ; \mathrm{P}=0.56 ; 667$ participants; very low-quality evidence; Analysis 1.3.

Data on hospital readmissions were reported in two of 41 trials (Holyday 2012; Van den Berg 2015). In the trial by Holyday 2012 the groups receiving a protocol-driven pathway for the management of nutrition whilst in hospital had fewer hospital readmissions than the group receiving usual care $(30 / 71$ versus $37 / 72$ respectively). However the between-group difference was not statistically significant. In the trial by Van den Berg 2015 there were more hospital readmissions in the group receiving an oral nutritional supplement four times daily than the groups receiving the supplement twice daily or the usual care group (24 versus 13 versus 15 respectively).

The trial by Potter 2001 reported the destination of participants at discharge according to group allocation. There was no marked difference between groups in the numbers of participants returning to their own home and those being discharged to an institution (Table 25).

\section{Adverse events}

Three of 41 trials (Dennis 2005; Faxen-Irving 2011; Hankey 1993) reported on adverse events, all trials evaluating the impact of supplementation of meals with oral nutritional supplements. The overall quality of the evidence was very low. The trial by FaxenIrving 2011 reported that 5 of 34 (15\%) participants experienced intolerance to the supplement assessed as diarrhoea and vomiting. In the trial by Dennis 2005565 of 2017 (28\%) of participants stopped taking the oral nutritional supplement due to individuals' refusal or dislike of taste, unwanted weight gain, or feelings of nausea. The trials by Potter 2001 and Van den Berg 2015 reported that no adverse events occurred.

\section{All-cause mortality}

Adequate data were reported on this outcome in 12 out of 41 trials (Bouillanne 2013; Brouillette 1991; Dennis 2005; Duncan 2006; Hickson 2004; Holyday 2012; Kretser 2003; Larsson 1990; Munk 2014; Olofsson 2007; Potter 2001; Van den Berg 2015). Six cluster-RCTs could not be included in the meta-analysis (BourdelMarchasson 2000; Leslie 2012; Mathey 2001a; Nijs 2006; Salva 2011; Smoliner 2008).

Trials were in participants from a variety of clinical backgrounds and in a range of different healthcare settings, receiving interventions which were all supportive of improved nutritional intake but varied widely. Meta-analysis showed a RR of 0.78 (95\% Cl 0.66 to 0.92$) ; \mathrm{P}=0.004 ; 12$ trials; 6683 participants; moderate-quality evidence; Analysis 1.4 in favour of supportive interventions (Figure 6). The test for subgroup differences of the various supportive interventions did not indicate interaction. Subgroup analysis of longer-term trials (four months to one year) showed a RR of 0.73 ( $95 \% \mathrm{Cl} 0.55$ to 0.98 ); 6 trials; 5200 participants. The sensitivity analysis after exclusion of the biggest trial, Dennis 2005, showed a RR of 0.67 (95\% CI 0.54 to 0.82); 11 trials; 2660 participants. 
Figure 6. Forest plot of comparison: 1 Supportive interventions for enhancing dietary intake versus comparators, outcome: 1.4 All-cause mortality

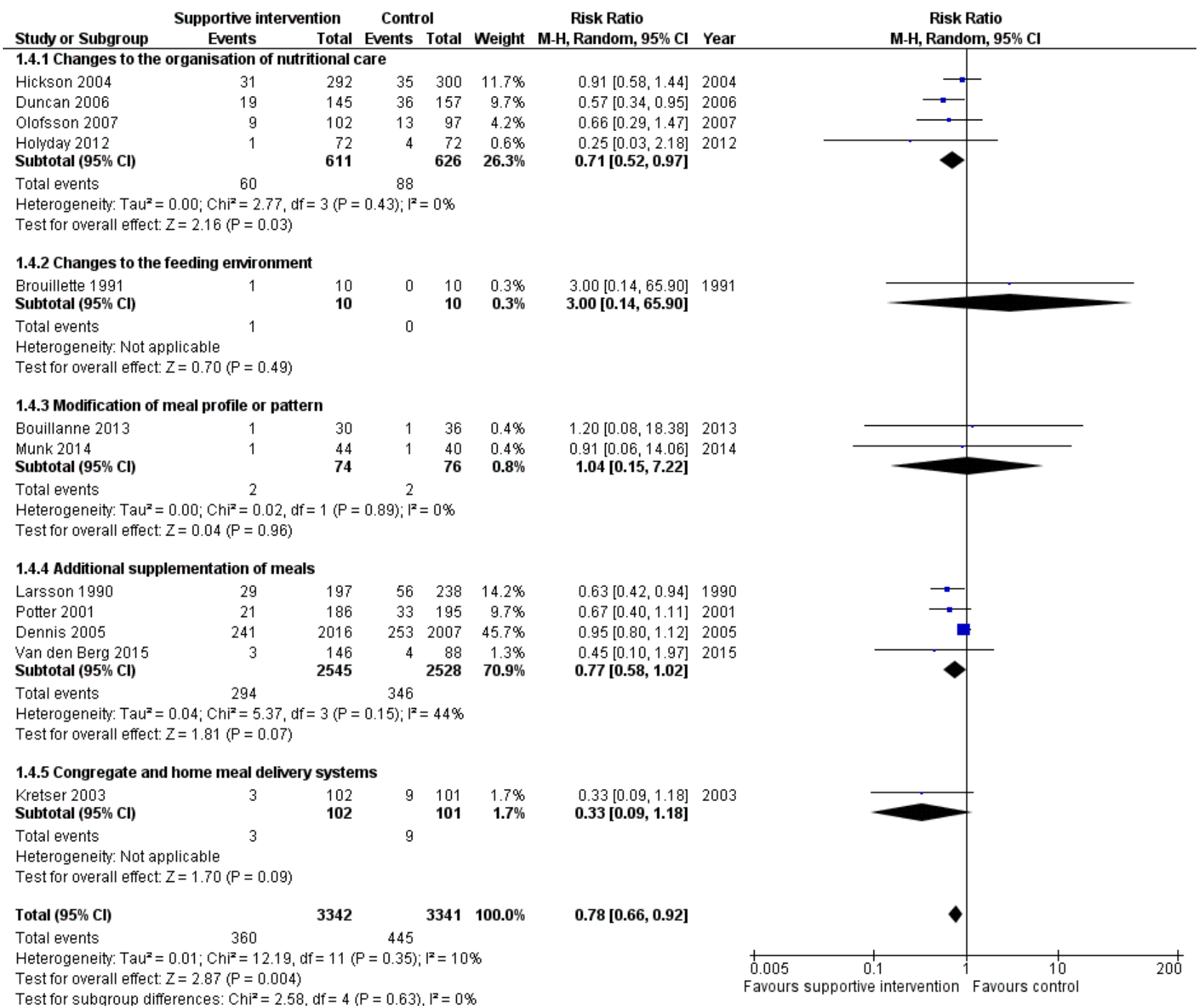

\section{Economic costs}

Data on this outcome were reported in three of 41 trials (Holyday 2012; Salva 2011; Simmons 2010). The overall quality of the evidence was very low. The trial by Holyday 2012 evaluated the impact of a protocol-driven pathway for the management of nutritional care in hospital patients and the trial by Salva 2011 evaluated the impact of specialist training for carers of free-living individuals with dementia. In the trial by Holyday 2012 the data on cost savings were based on reductions in the length of hospital stay. There was no marked difference in overall length of stay between groups. There was a shorter length of stay by eight days in the subgroup of 32 malnourished participants (12 days in the intervention group and 20 days in the usual care group). These data were used to estimate a cost saving of AUD 63,360 from treating malnutrition in the group of 12 malnourished participants based on the cost per hospital bed per day, the cost of the dietitians' time and the average cost of a commercial oral nutritional supplement. The trial by Salva 2011 collected data on resource utilisation but the data were not reported. The trial by Simmons 2010 evaluated the impact of a food-based and oral nutritional supplement-based

intervention. In this trial a formal cost effectiveness analysis was not undertaken and reporting of the impact of the interventions on costs was limited to a report of the cost per serving of the oral nutritional supplement or food provided and an estimate of staff time required to encourage and assist consumption. The average costs (per person per day in USD) were significantly higher in groups receiving supplements and snacks compared with those in the usual care group (USD 2.10 versus, USD 2.06). None of the trials used accepted health economic methods and the reported data on both costs and effectiveness were generally poor.

\section{Subgroup analyses}

We carried out the first planned subgroup analysis 'intervention category'. Trials were grouped according to similar interventions into five categories. There were insufficient data to undertake further subgroup analyses.

\section{Sensitivity analyses}

We did not do any sensitivity analyses because of insufficient data. 


\section{Changes to the organisation of nutritional care}

\section{Primary outcomes}

\section{Nutritional intake}

Data on energy intake were reported in five of 13 trials (Chang 2005; Duncan 2006; Hickson 2004; Johansen 2004; Lin 2010) (Table 10). Two trials used dietetic assistants in a hospital setting: one found a greater energy intake in groups receiving assistance than those receiving usual care (1105 kcal (SD 361) versus $759 \mathrm{kcal}$ (SD 399), $P<0.001$ ) (Duncan 2006), whereas in the other trial (Hickson 2004), which assessed between-group difference in intake in 37 of 592 participants, the difference in energy intake between the groups was $89 \mathrm{kcal}, \mathrm{P}<0.538$. Of the four trials that implemented specialist training in long-term care facilities, two reported data on energy intake as percentage of meals consumed (Chang 2005 ; Lin 2010). In one trial (Chang 2005), the intervention group experienced a reduction in percentage of meals consumed and the group receiving usual care increased their intake $(P<0.49)$. In the other trial (Lin 2010) there were small increases in percentage of meals consumed in all groups (Table 10). One trial providing multi-disciplinary team care in a hospital setting reported a greater energy intake in the intervention group compared with usual care (30 kcal $/ \mathrm{kg} / \mathrm{d}$ (standard error (SE) 1) versus $25 \mathrm{kcal} / \mathrm{kg} / \mathrm{d}$ (SE 1) (Johansen 2004).

\section{Health-related quality of life and patient satisfaction}

Data on health-related quality of life were reported in one of 13 trials (Johansen 2004). Quality of life was assessed using the SF36 questionnaire (Ware 1992) which was completed by $57 \%$ participants. A dropout analysis showed responders and nonresponders were similar in terms of baseline characteristics. There were no marked differences between the groups in both the physical and mental summary scores from baseline to follow-up (physical score mean 2.4 (SE 1.3) in the intervention versus mean 0.2 (SE 1.5) in the control; mental score mean 2.2 (SE 2.5) in the intervention versus mean 3.3 (SE 2) in the usual care) (Table 11).

Data on patient satisfaction were reported in two of 13 trials (Duncan 2006; Salva 2011). In the trial by Duncan 2006 patient satisfaction was assessed using an unvalidated questionnaire with 10 questions about aspects of meals, diet and feeding. Patients answered yes or no where yes $=1$, no $=-1$ and NA $=0$. Those participants who had received the support of the dietetic assistants showed greater satisfaction with a median score of 6.5 (IQR 2) compared to 3 (IQR 4) for participants receiving usual care $(P<0.0001)$ (Table 11). In the trial by Salva 2011 satisfaction of participants and their families was assessed using an unvalidated questionnaire which asked about the use of and perceived usefulness of five aspects of the overall programme. Families and carers were asked to indicate whether they had used the service and whether they had found it very useful, useful or not very useful. Information cards were used by $94.5 \%$ of families and rated as very useful (26\%) and useful (67\%). The nutrition course was used by $66 \%$ of families and rated as very useful $(24 \%)$ and useful $(65 \%)$. Weight curves were sent to $88 \%$ of families and rated as very useful (13\%) and useful (78\%). Information sessions were attended by $75 \%$ of families and rated as very useful $(32 \%)$ and useful ( $62 \%)$. The hot line was used by $33 \%$ of families and rated as very useful (17\%) and useful (51\%).

\section{Morbidity/complications}

Data on complications were reported in four of 13 trials (Duncan 2006; Hickson 2004; Johansen 2004; Olofsson 2007), three of which reported the number of participants experiencing any complications (Dennis 2005; Johansen 2004; Olofsson 2007) and one trial (Hickson 2004) reported the number of participants receiving oral antibiotics. There were no marked between-group differences in any of the trials (Table 11).

\section{Secondary outcomes}

\section{Nutritional status}

\section{Weight change}

Data on this outcome were reported in 10 of 13 trials (Duncan 2006; Hickson 2004; Holyday 2012; Johansen 2004; Kraft 2012; Lin 2010; Olofsson 2007; Pivi 2011; Salva 2011; Splett 2003) (Table 12).

Two trials evaluated the impact of dietetic assistants in a hospital setting (Duncan 2006; Hickson 2004) and there were no marked differences in mean weight change between groups in either trial. One trial used specialist training in a residential care setting (Lin 2010) and there was no marked difference in mean weight change between the two groups. Two trials looked at specialist training for carers of free-living individuals with dementia (Pivi 2011; Salva 2011). In one trial the intervention group experienced a small weight gain of $1.2 \mathrm{~kg}$ whereas the usual care experienced a small weight loss of $2.2 \mathrm{~kg}$ (Pivi 2011). In the other trial (Salva 2011) there was no marked difference between the two groups in mean weight change. Two trials reported weight change for interventions consisting of a multi-disciplinary team approach to nutritional care (Johansen 2004; Olofsson 2007) and reported no marked differences between groups receiving intervention and those receiving usual care in either trial. One trial described a protocol-driven pathway of nutritional care in hospital (Holyday 2012) and reported no marked differences in weight change between the groups receiving the intervention and usual care. Another trial reported data using a protocol-driven care in a care home setting (Splett 2003). The authors did not report mean weight change but provided a narrative description of the proportions of participants maintaining or gaining weight. The percentage of participants maintaining or gaining weight during the trial was greater in the usual care group (57\%) than in the intervention group (48\%). One trial evaluated the impact of telemedicine in free-living individuals and reported no marked difference between the groups in mean weight change (Kraft 2012).

\section{Change in BMI}

Data on this outcome were reported in seven of 13 trials (Hickson 2004; Kraft 2012; Lin 2010; Lin 2011; Olofsson 2007; Pivi 2011; Salva 2011): two trials of specialist training in a residential care setting (Lin 2010; Lin 2011), two of specialist training of free-living individuals (Pivi 2011; Salva 2011), one of additional nutritional care from a trained health care assistant (Hickson 2004), one of multi-disciplinary team care in hospital (Olofsson 2007) and one of telemedicine (Kraft 2012). There were no marked differences in BMI change between groups in six of the seven trials (Table 12). In one trial (Pivi 2011) participants receiving specialist training experienced an increase in BMI $\left(1.2 \mathrm{~kg} / \mathrm{m}^{2}\right.$ (SD 1) and participants in the usual care group experienced a reduction in BMI $\left(-2.2 \mathrm{~kg} / \mathrm{m}^{2}\right.$ (SD 1). However, the between-group difference and statistical tests were not reported. 


\section{Change in TSF, MAMC and MUAC}

Data on this outcome were reported in three of 13 trials (Duncan 2006; Hickson 2004; Pivi 2011). In the two trials that assessed the effects of using dietetic assistants in hospital (Duncan 2006; Hickson 2004) there were no marked differences in either TSF or MAMC between groups. In one trial (Hickson 2004) there was no marked difference in MAC between groups receiving assistance with eating and those receiving usual care, whereas in the other trial (Duncan 2006) the group that received assistance with eating had a smaller reduction in MAC $(-0.9 \mathrm{~cm}$ (SD 2.2)) compared with the group that received usual care $(-1.3$ (SD 1.5), $\mathrm{P}<0.002)$. One trial used specialist training in free-living individuals (Pivi 2011) and reported improvements in MAC in the intervention group of $1.9 \mathrm{~cm}$ (SD 2) compared with a reduction of $0.4 \mathrm{~cm}$ (SD 0.5) in the group receiving usual care, and no marked difference between the groups in TSF.

Overall the data across all interventions suggest that there is minimal impact on weight change and body composition from changes to the organisation of nutritional care across different healthcare settings.

\section{Clinical function}

Data on this outcome were reported in three of 13 trials (Duncan 2006; Hickson 2004; Salva 2011). The trials by Duncan 2006 and Hickson 2004 both assessed the effect of assistance with eating in people in hospital on handgrip strength. There were no marked differences in handgrip strength between the intervention and usual care groups in either trial (Table 13). The trial by Hickson 2004 also assessed functional recovery in participants using the Barthel score. There was no marked difference between groups' initial assessment to discharge from hospital (median score 2.0 (IQR 0 to 5 ) in the group receiving feeding assistance and 1.0 (IQR 0 to 4), $P=0.23$ in the group receiving usual care). The trial by Salva 2011 measured change in ADL (Katz 1963), and ADL (Lawton 1969) in free-living individuals with dementia who had received specialist training on nutrition. There were no marked differences between the groups in either ADL or iADL at six and 24 months' follow-up.

\section{Hospitalisation and institutionalisation}

Data were reported on length of hospital stay in five of 13 trials (Duncan 2006; Hickson 2004; Holyday 2012; Johansen 2004; Olofsson 2007). Two trials evaluated the impact of dietetic assistants in a hospital setting (Duncan 2006; Hickson 2004), two evaluated a multi-disciplinary team intervention in hospital (Olofsson 2007; Johansen 2004) and one evaluated a protocoldriven pathway in hospital (Holyday 2012). There were no marked differences between groups in length of hospital stay in four trials (Duncan 2006; Hickson 2004; Holyday 2012; Johansen 2004). In the other trial (Olofsson 2007) the group receiving a multidisciplinary team intervention had a shorter mean length of hospital stay than the group receiving usual care (27.4 days (SD 15.9) in the intervention group and 39.8 days (SD 41.9) in the usual care group $(P<0.05)$ (Table 14). Data on hospital readmissions were reported in one of 13 trials (Holyday 2012). The group receiving a protocol-driven pathway for the management of nutrition whilst in hospital had fewer hospital readmissions than the group receiving usual care $(30 / 71(42 \%)$ versus $37 / 72(51 \%)$ respectively) but the difference between the groups was not statistically significant.

\section{Adverse events}

No trial reported data on this outcome.

\section{All-cause mortality}

Data were reported on this outcome in five of 13 trials (Duncan 2006; Hickson 2004; Holyday 2012; Olofsson 2007; Salva 2011). Two trials evaluated the impact of dietetic assistants in a hospital setting (Duncan 2006; Hickson 2004), one evaluated specialist training for free-living individuals with dementia (Salva 2011), one evaluated a multi-disciplinary team intervention in hospital (Olofsson 2007) and one evaluated a protocol-driven pathway in hospital (Holyday 2012). There were no marked differences between groups in mortality in four trials (Hickson 2004; Holyday 2012; Olofsson 2007; Salva 2011), whereas in the other trial (Duncan 2006) there was a lower mortality at four months in the group receiving the intervention from dietetic assistants compared with the group receiving usual care (19/145 (13\%) versus 36/157 (23\%), $\mathrm{P}=0.036$ ) (Table 14).

\section{Economic costs}

Data on this outcome were reported in two of 13 trials (Holyday 2012; Salva 2011). One trial (Holyday 2012) evaluated the impact of a protocol-driven pathway for the management of nutritional care in hospital patients and the other trial (Salva 2011) evaluated specialist training for carers of free-living individuals with dementia. In one trial (Holyday 2012) the data on cost savings are based on reductions in length of stay achieved. There was no marked difference in length of stay overall between groups. There was a shorter length of stay by eight days in the subgroup of 32 malnourished participants ( 12 in the intervention group and 20 in the usual care group). These data were used to estimate a cost savings of AUD 63,360 from treating malnutrition in the group of 12 malnourished participants based on the cost per hospital bed per day, the cost of the dietitians' time and the average cost of a commercial oral nutritional supplement. The trial by Salva 2011 collected data on resource utilisation but the data were not reported. Neither trial used accepted health economic methods and the reported data on both costs and effectiveness were generally poor.

\section{Changes to the feeding environment}

\section{Primary outcomes}

\section{Nutritional intake}

Data were reported on energy intake in three of five trials (Brouillette 1991; Mathey 2001a; Nijs 2006). Two trials evaluated the impact of changes to the dining room environment (Mathey 2001a; Nijs 2006) and one evaluated a pre-meal sensory stimulation intervention (Brouillette 1991). All trials assessed energy intake and were conducted in people in residential care. There were no marked between-group differences in energy intake in any trial (Table 15).

\section{Health-related quality of life and patient satisfaction}

Data were reported on health-related quality of life in two of five trials (Mathey 2001a; Nijs 2006). One trial (Mathey 2001a) used the Sickness Impact Profile (SIP) (Gilson 1975), and Philadelphia Geriatric Center Morale Scale (PGCMS, 17 items) (Lawton 1972) to assess health-related quality of life.The SIP is a validated generic health status measure of change in behaviour as a consequence of illness. It includes 136 items describing activities of daily 
living ( $A D L)$, divided into 12 categories: sleep and rest, eating, work, home management, recreation and pastimes, ambulation, mobility, body care and movement, social interaction, alertness behaviour, emotional behaviour, and communication. Patients endorse statements that best describe them that day and are related to their health. Items are scored on a numeric scale, with higher scores reflecting greater dysfunction. The mean SIP score in the usual care declined more $(-13 \%$ (SD 12), $\mathrm{P}<0.05)$ than in the experimental group (-2\% (SD 11)). The PGCMS is a multidimensional approach to assessing the state of psychological well-being of older people. It measures perceived morale in elderly people through three factors: agitation, attitude toward own aging and 'lonely satisfaction'. Each high-morale response receives a score of ' 1 ' and each low-morale response a score of ' 0 ', so that the total score ranges from 0 to17. As a general guideline, scores between 13 to17 would be considered high scores on the morale scale, 10 to 12 fall within the mid-range and scores under 9 are at the lower end. Mean changes in the PGCMS scores were relatively stable for both groups with $-2 \%$ (SD 19) for the usual care, and -3\% (SD 20) for the experimental group. In the trial by Nijs 2006, health-related quality of life was assessed in a face-to-face interview using the Dutch health-related quality of life of somatic nursing home residents questionnaire which is a validated questionnaire consisting of five sub-scales, each representing a quality of life dimension: sensory functioning (focusing on pain); physical functioning (perceived performance and self-care); psychosocial functioning (depression or loneliness); perceived autonomy (freedom of movement); and perceived safety (feeling at home in the institution). The number of statements in the five sub-scales is not equal. The questionnaire consists of 50 statements, scored on a dichotomous scale (yes or no). Each subscale and the total questionnaire is computed to achieve a score from 0 to 100 . A high score represents a high quality of life. There was a difference between groups in overall quality of life (6.1 units, $95 \% \mathrm{Cl} 2.1$ to 10.3$)$. The intervention group remained stable $(0.4$ units, $95 \% \mathrm{Cl} 1.8$ to 2.5$)$, whereas the usual care declined (-0.5 units, $95 \% \mathrm{Cl}-9.4$ to 0.6 ), although the overall changes were small (Table $16)$.

No trial reported data on patient satisfaction.

\section{Morbidity/complications}

No trial reported data on this outcome.

\section{Secondary outcomes}

\section{Nutritional status}

\section{Weight change}

Data were reported on this outcome in three of five trials (Mathey 2001a; Nijs 2006; Remsburg 2001), all of which were trials evaluating the impact of changes to the dining environment. There were no marked differences between intervention and usual care groups in mean weight change in any of the trials (Table 17).

\section{Change in BMI}

No trial reported data on this outcome.

\section{Change in TSF}

No trial reported data on this outcome.

\section{Change in MAC}

Data were reported on this outcome in one of five trials (Nijs 2006). The trial evaluated the impact of providing family-style meals in residential care homes. There was no marked difference in change in MAC between the groups, MD between groups was $0.5 \mathrm{~cm}(95 \%$ $\mathrm{Cl}-0.2$ to 1.3 )

\section{Clinical function}

No trial reported data on this outcome.

\section{Hospitalisation and institutionalisation}

No trial reported data on this outcome.

\section{Adverse events}

No trial reported data on this outcome.

\section{All-cause mortality}

Data were reported on this outcome in three of five trials (Brouillette 1991; Mathey 2001a; Nijs 2006). Two evaluated the impact of changes to the dining room environment (Mathey 2001a; Nijs 2006) and one of pre-meal sensory stimulation (Brouillette 1991). There were no marked differences between groups in death from any cause in any trial (Table 18).

\section{Economic costs}

No trial reported data on this outcome.

\section{Modification of meal profile or pattern}

\section{Primary outcomes}

\section{Nutritional intake}

Data were reported on energy intake in 11 of 12 trials (Barton 2000; Bouillanne 2013; Castellanos 2009; Essed 2007; Essed 2009; Germain 2006; Leslie 2012; Mathey 2001b; Munk 2014; Silver 2008; Taylor 2006). Four trials evaluated the impact of food fortification, two in hospital (Barton 2000; Munk 2014), one in a care home (Leslie 2012) and one in free-living individuals receiving home-delivered meals (Silver 2008), one trial evaluated the impact of modifications to meal delivery in an intermediate care home (Bouillanne 2013), two trials evaluated modifications to meal delivery in residential care homes (Germain 2006; Taylor 2006), and three evaluated flavour modification in residential care homes (Essed 2007; Essed 2009; Mathey 2001b). There were no marked differences in mean change in energy intake between groups in five trials (Bouillanne 2013; Essed 2007; Essed 2009; Mathey 2001b; Taylor 2006). Three trials reported higher energy intakes in the intervention group of between 300 to $500 \mathrm{kcal} /$ day, two of which were trials of food fortification in either hospital or in free-living individuals (Barton 2000; Silver 2008) and one was of a modification to meal delivery involving improved presentation of pureed foods to participants with dysphagia (Germain 2006). In the randomised cross-over trial by Castellanos 2009, between-group differences were not reported however data were presented for a post hoc analysis of 'big' eaters (overall intake $1150 \mathrm{kcal}$ or more a day) and 'small' eaters (overall intake less than $1150 \mathrm{kcal}$ a day) (data not reported in the table). Data were presented as mean intake from both fortified and non-fortified food items at each meal under each of three menu conditions (Table 19). 


\section{Health-related quality of life and patient satisfaction}

Data on health-related quality of life were reported in one trial (Smoliner 2008). The physical functioning component of the validated medical outcomes Study 36-item Short Form (SF-36) were reported (Ware 1992). The SF-36 is a participant-completed validated questionnaire to assess eight different domains of health (vitality, physical functioning, bodily pain, general health perception, physical function, emotional role function, social role function and mental health). The SF-36 consists of eight scaled scores, which are the weighted sums of the questions in their section. Each scale is directly transformed into a 0 to 100 scale on the assumption that each question carries equal weight. The lower the score the poorer the quality of life. The higher the score the better the quality of life, that is, a score of zero is equivalent to poorest quality of life and a score of 100 is equivalent to optimal quality of life.

Baseline to follow-up (12 weeks) score in the intervention group receiving the fortified diet changed from a mean of 17.1 (SD 22.7) at baseline to a mean of 10.7 (SD 15.6) at 12 weeks $(P=0.047)$, and in the usual care from 24 (SD 24.3) at baseline to 13.6 (SD 13.9) at 12 weeks $(P<0.0001)$, however the between-group differences were not statistically significant.

No trial reported data on patient satisfaction.

\section{Morbidity/complications}

Data on the number of participants experiencing complications were reported in one of twelve trials (Bouillanne 2013) which evaluated the impact of modifications to meal composition in people in intermediate care. There was no marked difference between the intervention and usual care in the number of infectious complications experienced by participants included in the intention-to-treat analysis (1 of 29 participants in the intervention group and 2 of 34 participants in the usual care group).

\section{Secondary outcomes}

\section{Nutritional status}

\section{Weight change}

Data on this outcome were reported in seven of 12 trials (Bouillanne 2013; Essed 2007; Germain 2006; Leslie 2012; Mathey 2001b; Munk 2014; Smoliner 2008). Three trials evaluated the impact of food fortification, one in hospital (Munk 2014) and two in a residential care home (Leslie 2012; Smoliner 2008), one evaluated modification to meal composition in an intermediate care setting (Bouillanne 2013), one evaluated modifications to the presentation of food in a residential care home (Germain 2006) and two evaluated flavour modifications in residential care homes (Essed 2007; Mathey 2001b). There were no marked differences in mean weight change between groups reported in three trials (Bouillanne 2013; Essed 2007; Smoliner 2008). Three trials reported higher weight gain in the intervention group compared with the usual care. One was a trial of food fortification in residential care (Leslie 2012) (1.3 kg (SE 0.53) in the intervention group versus $-0.2 \mathrm{~kg}$ (SE 1.5) in the control group, $P=0.03$. The second was a trial of modification to meal presentation (Germain 2006) (3.9 kg (SD 2.3) in the intervention group versus $-0.8 \mathrm{~kg}$ (SD 4.2) in the usual care. The other trial evaluated the impact of flavour enhancement in people in a residential care home (Mathey 2001b) $(1.1 \mathrm{~kg}$ (SD 1.3) in the intervention group versus $-0.3(1.6)$ in the usual care, $\mathrm{P}<0.05$ ) (Table 20).

\section{Change in BMI}

Data on this outcome were reported in three of 12 trials (Germain 2006; Leslie 2012; Smoliner 2008). One evaluated the impact of modification to meal presentation in people in residential care (Germain 2006) and the others evaluated food fortification in people in residential care (Leslie 2012; Smoliner 2008). In one trial (Smoliner 2008) there was no marked difference between the groups in change in BMI. The group receiving modification to the presentation of meals in Germain 2006 and the group receiving fortified meals in Leslie 2012 experienced a greater increase in BMI than those receiving usual care but the between-group difference was not reported (Table 20).

\section{Change in TSF}

No trial reported data on this outcome.

\section{Change in MAC}

One trial of meal fortification in people in residential care reported data on this outcome (Leslie 2012). Participants in the intervention group experienced a greater improvement in MUAC than those in the control group (mean change $0.4 \mathrm{~mm}$ (SE 0.16) in the intervention group and $-0.1 \mathrm{~mm}$ (SE 0.3) in the control group, $\mathrm{P}=$ 0.019 .

\section{Clinical function}

Data on handgrip strength were reported in three of 12 trials (Bouillanne 2013; Munk 2014; Smoliner 2008). One trial evaluated the impact of modification to meal composition in people in intermediate care (Bouillanne 2013) and the others evaluated food fortification in people in hospital (Munk 2014) and in residential care (Smoliner 2008). There were no differences between the intervention and usual care groups in either trial (Table 21). The trial by Bouillanne 2013 also assessed change in ADL score (Sonn 1996) and there was no marked difference between the groups (Table 21). In the trial by Smoliner 2008 clinical function was also assessed by peak flow and the Barthel index. The peak flow (L/min) in the intervention group increased from baseline to follow-up (12 weeks) in the intervention group (mean 152 (SD 105) to 186 (SD 140)) whereas the usual care group showed a decline (mean 151 (SD 90) to 150 (SD 67)). The differences observed between groups were statistically significant $(P=0.039)$. The mean change in Barthel score was -15.2 (SD 18.5) in the group receiving fortification of food and -7.5 (SD 10.4) in the group receiving usual care. The betweengroup differences were not statistically significant.

\section{Hospitalisation and institutionalisation}

One trial of food fortification of menu items provided via an a la carte menu reported data on length of hospital stay (Munk 2014). There were no differences in mean length of stay between groups in from trial inclusion to discharge from hospital (mean 10 days (SD 8 ) in the intervention group and mean 10 days (SD 8) in the control group, between-group difference, 0.6 days $(95 \% \mathrm{Cl}-3$ to $4, \mathrm{P}=0.73)$.

\section{Adverse events}

No trial reported data on this outcome.

\section{All-cause mortality}

Data on this outcome were reported in four of 12 trials (Bouillanne 2013; Leslie 2012; Munk 2014; Smoliner 2008). The number of 
deaths were small in each trial and there were no marked differences between groups (Table 21).

\section{Economic costs}

No trial reported data on this outcome.

\section{Additional supplementation of meals}

\section{Primary outcomes}

\section{Nutritional intake}

Data were reported on energy intake in eight of 10 trials (Beck 2002; Bourdel-Marchasson 2000; Faxen-Irving 2011; Hankey 1993; Potter 2001; Simmons 2008; Simmons 2010; Van den Berg 2015). Three trials evaluated the impact of supplementation with food in residential care homes (Beck 2002; Simmons 2008; Simmons 2010), four evaluated supplementation with oral nutritional supplements in hospital (Bourdel-Marchasson 2000; Faxen-Irving 2011; Potter 2001; Van den Berg 2015) and two evaluated supplementation with oral nutritional supplements in residential care homes (Hankey 1993; Simmons 2010). One trial provided both a food-based intervention and oral nutritional supplements in participants in residential care homes (Simmons 2010). There were no marked differences reported in energy intake between groups in either the trials of food-based interventions or the trials of oral nutritional supplement-based interventions (Table 22). In the trial by (Hankey 1993) the group receiving oral nutritional supplements had an energy intake $600 \mathrm{kcal}$ greater than the usual care group (1747 kcal (SD 273) versus 1147 kcal (SD 310) respectively), However, between-group statistical tests were not reported. In the trial by Van den Berg 2015 participants receiving oral nutritional supplements in four $62 \mathrm{~mL}$ portions during the drug round had a significantly higher energy intake than those receiving supplements in the conventional, between-meal style.

\section{Health-related quality of life and patient satisfaction}

Data on health-related quality of life were reported in one trial (Dennis 2005) undertaken in people with stroke supplemented with oral nutritional supplements during hospitalisation. Healthrelated quality of life was measured in $77 \%(\mathrm{~N}=3086)$ of participants using EUROQoL score (EQ-5D) (EuroQol group 1990). The questionnaire comprises five questions on mobility, self-care, pain, usual activities and psychological status with three possible answers for each item ( 1 = no problems, 2 = moderate problems, 3 $=$ severe problems). An overall utility score is calculated based on these domains, with a range score from 0 (worse health scenario) to a maximum of 1.0 (best health scenario). An additional visual analogue scale (VAS, scale 0 to 100) was used to assess general health status with 100 indicating the best health status. No marked differences were identified between the intervention and usual care groups (Table 23).

No trial reported data on patient satisfaction.

\section{Morbidity/complications}

The incidence of, and number of people with, pressure ulcers was reported in two trials (Bourdel-Marchasson 2000; Dennis 2005) and the total number of complications was reported in one trial (Dennis 2005). Both trials were of supplementation of participants with oral nutritional supplements in hospital. There was no marked difference between groups in cumulative incidence of, or number of participants with, pressure ulcers in either trial (Table 23). In the trial by Dennis 2005 there was no marked difference in total complications between groups (Table 23).

\section{Secondary outcomes}

\section{Nutritional status}

\section{Weight change}

Data on this outcome were reported in seven of 10 trials (Beck 2002; Faxen-Irving 2011; Hankey 1993; Larsson 1990; Potter 2001; Simmons 2008; Simmons 2010). Three trials evaluated the impact of supplementation with food in residential care settings (Beck 2002; Simmons 2008; Simmons 2010), two evaluated supplementation with oral nutritional supplements in hospital (Faxen-Irving 2011; Potter 2001) and three evaluated supplementation with oral nutritional supplements in long-term care settings (Hankey 1993; Larsson 1990; Simmons 2010), with the trial by Simmons 2010 providing data on both food and oral nutritional supplements. There were no marked differences in weight change between groups receiving food-based or oral nutritional supplement-based interventions in six trials (Beck 2002; Faxen-Irving 2011; Hankey 1993; Larsson 1990; Potter 2001; Simmons 2010). In two trials (Faxen-Irving 2011; Hankey 1993), the groups receiving oral nutritional supplements gained weight and the usual care group lost weight overall. However, the betweengroup differences and the results of statistical tests were not reported. In one trial (Simmons 2008) the intervention group gained $4 \mathrm{lbs}$ more in weight than the group receiving usual care ( $P$ $=0.009$ ) (Table 24).

\section{Change in BMI}

Data on this outcome were reported in two of 10 trials (FaxenIrving 2011; Simmons 2008), both trials evaluated the impact of supplementation with oral nutritional supplements in hospital. In one trial (Faxen-Irving 2011) BMI was reported according to group at the end of the intervention and there was no marked difference between groups. Change from baseline and betweengroup differences were not reported. In the other trial by (Simmons 2008) the intervention group gained $0.72 \mathrm{~kg} / \mathrm{m}^{2}$ more than the group receiving usual care $(\mathrm{P}<0.009)$ (Table 24).

\section{Change in TSF}

Data on this outcome were reported in two of 10 trials (Hankey 1993; Larsson 1990), both of which evaluated the impact of supplementation with oral nutritional supplements in long-term care settings. In each trial data were presented in figures with minimal description in the text. In one trial (Hankey 1993) the intervention group was described as experiencing a smaller decrease in TSF than the usual care group (6.6\% versus $15.8 \%)$. In the other trial (Larsson 1990) TSF decreased over the 26 weeks of follow-up with the greatest decrease occurring in the usual care group. In another trial (Potter 2001) TSF is described as an outcome but the data were not reported.

\section{Change in MACe}

Data on this outcome were reported in three of 10 trials (Hankey 1993; Larsson 1990; Potter 2001), all of which evaluated the impact of supplementation with oral nutritional supplements in either hospital or long-term care settings. In one trial (Hankey 1993), the data were unavailable from the original trial report but we have obtained them from a systematic review by Milne 2009. We read the figures for change from a graph and assumed SD of change to be 
$10 \mathrm{~cm}$ for each group. MAC is described as improving statistically significantly in the intervention group $(P<0.05)$ but remaining unchanged in the usual care group. The changes are small and no between-group differences were reported (Table 24). In the trial by Larsson 1990 the data were presented in a figure with some description in the text, participants who were well nourished at the start of the trial and received supplementation of meals experienced less of a decrease in MAC at 26 weeks $(P<0.05)$ than those receiving usual care. In participants who were malnourished at the start of the trial both groups experienced a decrease in MAC to 26 weeks. In the final trial (Potter 2001), there was no marked difference between groups in MAC (Table 24).

\section{Clinical function}

Data on clinical function were reported in two of ten trials (Faxen-Irving 2011; Potter 2001), both evaluating the impact of supplementation with oral nutritional supplements in hospital. In one trial (Faxen-Irving 2011) the group receiving oral nutritional supplements changed from being dependent in all five functions to being dependent in only one function as assessed by ADL (Katz 1963). However, no marked change was identified in those receiving usual care $(P=0.011)$. Mean change $(S D)$ in $A D L$ score according to group was not markedly different between groups (2.95 (SD 2.2) intervention and 4.1 (SD 2.2) control, $P=0.09$ ). In the other trial (Potter 2001) there was no statistically significant difference in numbers achieving functional recovery assessed using the Barthel index in the group receiving supplementation compared with the usual care group (102/149 (68\%) intervention versus $100 / 157(64 \%)$ control, $P=0.38)$. However, significantly more participants classified as severely undernourished experienced an improvement in their Barthel scores on supplementation compared with those who received usual care $(17 / 25(68 \%)$ intervention versus $11 / 28(39 \%)$ control, $\mathrm{P}<0.04)$.

\section{Hospitalisation and institutionalisation}

Data on length of hospital stay were reported in four of 10 trials (Dennis 2005; Faxen-Irving 2011; Potter 2001; Van den Berg 2015) all of which evaluated the impact of supplementation of meals with oral nutritional supplements in hospital. There were no marked differences in length of hospital stay between groups in any trial (Table 25).

One trial of supplementation with oral nutritional supplements in hospital reported data on hospital re-admissions (Van den Berg 2015). The number of re-admissions to hospital were higher in intervention group 2, but these data were not commented on by the trial authors (13 participants in intervention group 1, 24 participants in intervention group 2 and 15 participants in the control group being readmitted to hospital). One trial reported on the destination of participants at discharge according to group allocation (Potter 2001). There was no marked difference between groups in numbers of participants returning to their own home and those being discharged to an institution (Table 25).

\section{Adverse events}

Data on this outcome were reported in three of nine trials (FaxenIrving 2011; Hankey 1993; Dennis 2005), one of which reported intolerance to the oral nutritional supplement (e.g. diarrhoea or vomiting, $\mathrm{N}=5$ ) (Faxen-Irving 2011). Another trial (Dennis 2005) reported that $28 \%$ stopped taking the oral nutritional supplement due to participant refusal or because of dislike of taste, unwanted weight gain, or feelings of nausea. The trials by Potter 2001 and Van den Berg 2015 reported no adverse events.

\section{All-cause mortality}

Data on this outcome were reported in five of 10 trials (BourdelMarchasson 2000; Dennis 2005; Larsson 1990; Potter 2001: Van den Berg 2015). Four trials evaluated the impact of supplementation with oral nutritional supplements in hospital (Bourdel-Marchasson 2000; Dennis 2005; Potter 2001; Van den Berg 2015 ) and one evaluated supplementation with oral nutritional supplements in a long-term care setting (Larsson 1990;). There was no marked difference in death from any cause between groups in any of the trials (Table 25).

\section{Economic costs}

Data on this outcome were reported in one trial (Simmons 2010). The cost effectiveness of the intervention was determined from data on cost per serving of the oral nutritional supplement or food provided and staff time to encourage and assist consumption. The average costs (per person per day) were significantly higher in groups receiving supplements and snacks compared with those in the usual care group (USD 2.10 versus USD 2.06 versus USD -0.03 respectively). The trial did not use accepted health economic methods and the reported data on both costs and effectiveness were generally poor.

\section{Home meal delivery systems}

\section{Primary outcomes}

\section{Nutritional intake}

No trial data were reported on this outcome.

Health-related quality of life and patient satisfaction

No trial data were reported on this outcome.

\section{Morbidity/complications}

No trial data were reported on this outcome.

\section{Secondary outcomes}

\section{Nutritional status}

\section{Weight change}

Data on this outcome were reported in the one trial in this group (Kretser 2003). The group receiving modified meals-on-wheels experienced a weight gain of $1.6 \mathrm{~kg}$ (SD 4.6) compared to the group receiving standard meals-on-wheels who had an overall weight gain of $0.7 \mathrm{~kg}$ (SD 3.3) (Table 26). No statistical tests were conducted on the between-group differences.

\section{Change in BMI}

No trial data were reported on this outcome.

\section{Change in TSF}

No trial data were reported on this outcome.

\section{Change in MAC}

No trial data were reported on this outcome. 


\section{Clinical function}

The one trial in this group reported data on ADL and iADL (Kretser 2003). No marked differences were identified in the number experiencing a decline $(4 / 22$ versus $8 / 24)$ or improvement $(3 / 22$ versus 2/24) in ADL between groups receiving modified mealson-wheels, and groups receiving traditional meals-on-wheels. However, there was a greater number of participants experiencing a decline in $\mathrm{iADL}$ in those receiving traditional meals on wheels $(16 / 24)$ compared with those receiving modified meals on wheels $(8 / 22)$ at six months $(P=0.0494)$.

\section{Hospitalisation and institutionalisation}

No trial data were reported on this outcome.

\section{Adverse events}

No trial data were reported on this outcome.

\section{All-cause mortality}

Data on this outcome were reported in the one trial in this group (Kretser 2003). The number of deaths from any cause were similar in each group (Table 26 ). No statistical tests were conducted on the between-group differences.

\section{Economic costs}

No trial reported data on this outcome.

\section{DISCUSSION}

\section{Summary of main results}

The aim of this review was to look for an effect of supportive interventions to enhance dietary intake in nutritionally vulnerable adults on patient-centred, nutritional, clinical and economic outcome. We identified 41 trials and categorised them into five broadly similar types of intervention. Meta-analysis was only possible for the outcome measures all-cause mortality, hospitalisation and nutritional status (weight change) showing a possible effect in favour of supportive dietary interventions for all-cause mortality and nutritional status. These findings should be interpreted with caution as few trials reported data on the outcomes of interest, and the quality of the evidence was between moderate to very low, depending on the outcome measurement. A number of patient-important outcomes were measured by just a few trials, for example, health-related quality of life and patient satisfaction. With regard to health-related quality of life only one of the five trials that reported this outcome suggested benefits associated with the intervention. Although the two trials that measured patient satisfaction reported benefits in those receiving the intervention it should be noted that both trials used unvalidated questionnaires and are potentially subject to the limitations inherent in collecting these types of data, for example, participants need to be literate to complete the questionnaire, blinding may not be possible.

Until there are more large trials of higher methodological quality, evaluating the impact of similar interventions in similar patient groups, the effects of supportive interventions on nutritional, clinical, patient-centred and healthcare outcomes cannot be fully evaluated.

\section{Overall completeness and applicability of evidence}

The trials identified for this review represent a wide range of interventions given with the aim of improving intake in nutritionally vulnerable individuals. Interventions took place in a variety of settings, residential care, hospital and outpatients. Although 21 of 41 included trials took place in residential care, the results of the meta-analyses were dominated by large trials conducted in hospitals. It is particularly important to consider that the relevance of different outcomes are likely to differ between settings; most of the data for the outcome of all-cause mortality came from trials recruiting hospital inpatients. Many of the interventions identified were similar to those recommended in policy and guideline documents on the prevention and management of malnutrition (BAPEN 2012; RCON 2008; The Malnutrition Task Force 2013). Despite the comprehensive range of interventions identified in this review, no RCTs were found for some widely used interventions, specifically protected meal times and the use of red trays to identify those requiring mealtime assistance. Examples of good practice reported in these key documents (BAPEN 2012; RCON 2008; The Malnutrition Task Force 2013) are frequently justified on the basis of their potential impact on patient experience and on staff awareness and motivation. These sorts of outcomes are rarely reported in trials, and therefore are not included in systematic reviews and meta-analyses. The key finding of this review is that there is a lack of evidence to support these interventions and good quality RCTs are urgently needed to inform the widespread implementation of these initiatives. While there is limited evidence on adverse events, nutritional interventions are generally assumed to be safe. However, the impact of implementing and maintaining such interventions at an organisational and unit level has not been evaluated. For example, there are likely to be significant costs in terms of finance, time and resources associated with setting up and maintaining a staff training programme, yet these data are rarely reported. In this review we found very limited data on costs and no formal health economic analyses from which to draw conclusions.

During searching for this review a number of trials were identified that met the inclusion criteria for types of participants and interventions, however they were non-randomised trials. The reasons for the weaker methodology used in many trials can only be speculated on, and may result from lack of funding, lack of research expertise, concern about the ethics of not providing all participants with an intervention perceived as 'beneficial', and practicalities related to the care setting. This underlines the need for adequate funding of trials with more robust designs (e.g. clusterrandomised controlled trials with adequate planning, analysis and data especially on intracluster correlation coefficients) to enable a fuller understanding of the potential impact of supportive interventions.

\section{Quality of the evidence}

The quality of evidence in this review is between moderate to very low, depending on the outcome measurement. The main issue regarding risk of bias was that although attrition was usually reported clearly and there was little evidence of selective reporting, random sequence generation, concealment of allocation and blinding were frequently unclear. Most trials were small and inadequately powered to answer the question. Although there was significant performance bias, the nature of the included interventions and the settings in which they were undertaken, primarily care homes and hospital wards, means that it is unlikely 
that participants in the usual care arms were able to get access to the intervention. The possible exceptions to this are the trials by Pivi 2011 and Salva 2011, where a training intervention was provided to carers of people with Alzheimers disease living at home. In this case, it might have been possible for the carers allocated to the usual care group to seek out the information provided to those in the intervention group. Interestingly, the effect size in the trial by Pivi 2011, was significantly different from others in that grouping.

A meta-analysis and GRADE approach was only possible for the outcome measures all-cause mortality, length of hospital stay and weight change. These outcomes showed moderatequality evidence (all-cause mortality, nutritional status) and very low-quality evidence (hospitalisation), mainly because of the small number of included trials and issues of imprecision and indirectness, as well as inconsistency.

\section{Potential biases in the review process}

The protocol developed prior to undertaking this review was followed closely, throughout the process and particularly during the trial selection stage when three review authors were involved in detailed discussion. The original search strategy for this review was comprehensive in that we searched 10 databases, including databases other than those most commonly used (Avenell 2001) and we did not place any language restrictions on searches. We undertook additional searching, for example hand searching of the abstracts of meetings, reference lists of identified trials and extensive searching of the reference lists of relevant systematic reviews. In addition, we made considerable efforts to contact authors of included studies, where clarification of data or methodology were required. However, we did not survey study authors to identify additional reports of trials that may have been missed, which has to be acknowledged as a potential source of bias.

There was considerable clinical heterogeneity across all trials contributing to the findings in this review. At the trial selection stage and during categorisation of trials into sub-groups, care was taken to group trials with similar interventions and populations together. It is possible that interventions judged to be similar, varied according to factors that are currently impossible to identify. For example, the trials evaluating the training of carers or dietetic assistants to deliver improved nutritional care resulted in different effects which may be attributable to a number of factors such as the quality of training, the level of attention provided by individual carers, constraints of the care setting, or indeed to the clinical characteristics of the trial populations. It was not possible to undertake many of the proposed subgroup analyses due to an absence of data. In addition, 12 of 41 (30\%) trials included in this review were cluster-randomised trials. Inadequate analysis methods used in these trials, which failed to account for the likelihood of similarity of participants within clusters and correlation of observations within clusters meant that these trials were excluded from the meta-analyses. We cannot rule out the possibility that inclusion of data from these 12 trials in the metaanalyses might change the overall findings.

\section{Agreements and disagreements with other studies or reviews}

The authors are aware of four published reviews of similar interventions (Cole 2012; Lambert 2010; Silver 2009; Weekes 2009), two of which employed systematic search strategies to identify trials (Cole 2012; Weekes 2009). All of the reviews looked at similar groupings of interventions (e.g. feeding assistance, changes to eating environment, staff training) and indeed included some of the trials identified in this review. They also included trials of weaker methodological quality (e.g. non-randomised controlled trials), excluded from this review.

One review (Weekes 2009) arrived at a similar conclusion to this one, that there was a serious lack of evidence to support interventions designed to improve nutritional care. The other three focused on positive results from individual trials.

To the review authors' knowledge, this is the first attempt at a systematic review with meta-analyses, the results of which reveals lack of good evidence for supportive interventions. While the protocol specified outcome measures that are frequently assessed in nutrition intervention trials, the review authors question whether these are the most appropriate outcomes to assess the benefits of supportive interventions. Existing reports of supportive interventions similar to the ones identified in this review, have speculated on their benefits in terms of patient experience, staff awareness and motivation. These may be more relevant outcome measures for interventions of this type, which may explain the lack of trials for interventions such as the use of red trays, or protected meal times, since the primary intention was to improve the patient experience.

The review authors note however, that the explicit aim of all the trials included in this review was to increase dietary intake, and thus influence clinical outcome.

\section{AUTHORS' CONCLUSIONS}

\section{Implications for practice}

There is moderate-quality evidence that supportive interventions to improve nutritional care improve nutritional status such as minimal weight gain or energy intake. Moderate-quality evidence shows that supportive interventions can reduce the risk of all-cause mortality, based mainly on studies recruiting hospital inpatients. There was very low-quality evidence to suggest adverse effects maybe associated with the interventions. Therefore, whilst some of these interventions are advocated at a national level, clinicians should recognise the lack of clear evidence to support their role across different settings.

\section{Implications for research}

This review revealed a lack of good quality randomised controlled trials evaluating the effect of supportive interventions. However, even small effects such as a potential reduction in all-cause mortality could result in relevant public health effects given the number of affected malnourished or nutritionally at-risk individuals. As these interventions remain in common use and are actively promoted at a national level, research is urgently needed. This review has identified a range of interventions that may benefit nutritionally vulnerable individuals and highlights the importance of assessing patient-important outcomes in different healthcare settings in future research.

The nature of the interventions being examined in the studies included in this review means that cluster-randomised trials are likely to be the method of choice because of the need to study the effects of interventions in groups of patients rather than 
individuals. Attention should be given to the reporting of clusterrandomised trials to take into consideration the correlation of observations within clusters and authors should account for the potential bias inherent in these trials when analysing and reporting results. Cluster level analyses, analyses of individual level data that are adjusted for the design effect, or regression analyses of individual level data using methods for clustered data are all valid approaches (McKenzie 2014).

\section{ACKNOWLEDGEMENTS}

We wish to thank Professor Peter Emery of King's College London, UK for his time and input into this review. We would also like to thank Karen Poole, Biomedical \& Health Information Specialist at King's College London, UK for her useful introduction to database searching in the protocol's early stages.

Additionally we are grateful to Dr Rafael Perera of the Department of Primary Healthcare Science, University of Oxford for his advice on statistical methods. We wish to thank all the staff and Editor of Cochrane Metabolic and Endocrine Disorders for their assistance in the conduct of this review. Particular thanks to the Co-ordinating Editor, Professor Bernd Richter and Maria-Inti Metzendorf, the Information Specialist of Cochrane Metabolic and Endocrine Disorders, who have made substantial contributions to identifying and interpreting the trials for this review. 


\section{R E F E R E N C E S}

\section{References to studies included in this review}

Barton 2000 \{published data only\}

Barton AD, Beigg CL, Macdonald IA, Allison SP. A recipe for improving food intakes in elderly hospitalized patients. Clinical Nutrition 2000;19(6):451-4.

\section{Beck 2002 \{published data only\}}

Beck AM, Ovesen L, Schroll M. Home-made oral supplement as nutritional support of old nursing home residents, who are undernourished or at risk of undernutrition based on the MNA. A pilot trial. Mini Nutritional Assessment. Aging Clinical and Experimental Research 2002;14(3):212-5.

\section{Bouillanne 2013 \{published data only\}}

* Bouillanne O, Curis E, Hamon-Vilcot B, Nicolis I, Chretien P, Schauer N, et al. Impact of protein pulse feeding on lean mass in malnourished and at-risk hospitalized elderly patients: a randomized controlled trial. Clinical Nutrition 2013;32(2):186-92.

Bouillanne O, Neveux N, Nicolis I, Curis E, Cynober L, Aussel C. Long-lasting improved amino acid bioavailability associated with protein pulse feeding in hospitalized elderly patients: a randomized controlled trial. Nutrition 2014;30(5):544-50. [PUBMED: 24355438]

\section{Bourdel-Marchasson 2000 \{published data only\}}

Bourdel-Marchasson I, Barateau M, Rondeau V, DequaeMerchadou L, Salles-Montaudon N, Emeriau J, et al. A multicenter trial of the effects of oral nutritional supplementation in critically ill older inpatients. Nutrition 2000;16(1):1-5.

\section{Brouillette 1991 \{published data only\}}

Brouillette ME, White LW. The effects of olfactory stimulation on the appetites of nursing home residents. Physical \& Occupational Therapy in Geriatrics 1991;10(1):1-13.

\section{Castellanos 2009 \{published data only\}}

Castellanos VH, Marra MV, Johnson P. Enhancement of select foods at breakfast and lunch increases energy intakes of nursing home residents with low meal intakes. Journal of the American Dietetic Association 2009;109(3):445-51.

\section{Chang 2005 \{published data only\}}

Chang CC, Lin LC. Effects of a feeding skills training programme on nursing assistants and dementia patients. Journal of Clinical Nursing 2005;14(10):1185-92.

\section{Dennis 2005 \{published data only\}}

Dennis M. Routine oral nutritional supplementation for stroke patients in hospital (FOOD): A multicentre randomised controlled trial. Lancet 2005;365(9461):755-63.

\section{Duncan 2006 \{published data only\}}

Duncan DG, Beck SJ, Hood K, Johansen A. Using dietetic assistants to improve the outcome of hip fracture: a randomised controlled trial of nutritional support in an acute trauma ward. Age and Ageing 2006;35(2):148-53.
Essed 2007 \{published data only\}

Essed NH, Van Staveren WA, Kok FJ, De Graaf C. No effect of 16 weeks flavor enhancement on dietary intake and nutritional status of nursing home elderly. Appetite 2007;48(1):29-36.

Essed 2009 \{published data only\}

Essed NH, Oerlemans P, Hoek M, Van Staveren WA, Kok FJ, De Graaf C. Optimal preferred MSG concentration in potatoes, spinach and beef and their effect on intake in institutionalized elderly people. Journal of Nutrition, Health \& Aging 2009;13(9):769-75.

\section{Faxen-Irving 2011 \{published data only\}}

Faxen-Irving G, Cederholm T. Energy dense oleic acid rich formula to newly admitted geriatric patients--feasibility and effects on energy intake. Clinical Nutrition 2011;30(2):202-8.

Gaskill 2009 \{published data only\}

Gaskill D, Isenring EA, Black LJ, Hassall S, Bauer JD. Maintaining nutrition in aged care residents with a train-the-trainer intervention and nutrition coordinator. Journal of Nutrition, Health \& Aging 2009;13(10):913-7.

\section{Germain 2006 \{published data only\}}

Germain I, Dufresne T, Gray-Donald K. A novel dysphagia diet improves the nutrient intake of institutionalized elders. Journal of the American Dietetic Association 2006;106(10):1614-23.

Hankey 1993 \{published data only\} Hankey CR, Summerbell J, Wynne HA. The effect of dietary supplementation in continuing-care elderly people: nutritional, anthropometric and biochemical parameters. Journal of Human Nutrition and Dietetics 1993;6(4):317-22.

\section{Hickson 2004 \{published data only\}}

Edington J, Barnes R, Bryan F, Dupree E, Frost G, Hickson M, et al. A prospective randomised controlled trial of nutritional supplementation in malnourished elderly in the community: clinical and health economic outcomes. Clinical Nutrition 2004;23(2):195-204. [PUBMED: 15030959]

* Hickson M, Bulpitt C, Nunes M, Peters R, Cooke J, Nicholl C, Frost $\mathrm{G}$. Does additional feeding support provided by health care assistants improve nutritional status and outcome in acutely ill older in-patients? - a randomised control trial. Clinical Nutrition 2004;23(1):69-77.

Hickson M, Frost G. An investigation into the relationships between quality of life, nutritional status and physical function. Clinical Nutrition 2004;23:213-21.

Hickson M, Nicholl C, Bulpitt C, Fry M, Frost G, Davies L. The design of the feeding support trial - does intensive feeding support improved nutritional status and outcome in older inpatients?. Journal of Human Nutrition \& Dietetics 1999;12:53-59.

Holyday 2012 \{published data only\}

Holyday M, Daniells S, Bare M, Caplan GA, Petocz P, Bolin T. Malnutrition screening and early nutrition intervention 
in hospitalised patients in acute aged care: a randomised controlled trial. Journal of Nutrition, Health \& Aging 2012;16(6):562-8.

\section{Johansen 2004 \{published data only\}}

Johansen N, Kondrup J, Plum LM, Bak L, Norregaard P, Bunch E, et al. Effect of nutritional support on clinical outcome in patients at nutritional risk. Clinical Nutrition 2004;23(4):539-50.

\section{Kraft 2012 \{published data only\}}

Kraft M, Van den Berg N, Kraft K, Schmekel S, Gartner S, Kruger J, et al. Development of a telemedical monitoring concept for the care of malnourished geriatric home-dwelling patients: a pilot study. Maturitas 2012;72(2):126-31.

\section{Kretser 2003 \{published data only\}}

Kretser AJ, Voss T, Kerr WW, Cavadini C, Friedmann J. Effects of two models of nutritional intervention on homebound older adults at nutritional risk. Journal of the American Dietetic Association 2003;103(3):329-36.

\section{Larsson 1990 \{published data only\}}

Ek AC, Unosson M, Larsson J, Von Schenck H, Bjurulf P. The development and healing of pressure sores related to the nutritional state. Clinical Nutrition 1991;10(5):245-50.

* Larsson J, Unosson M, Ek AC, Nilsson L, Thorslund S, Bjurulf P. Effect of dietary supplement on nutritional status and clinical outcome in 501 geriatric patients - a randomised study. Clinical Nutrition 1990;9(4):179-84.

Unosson M, Larsson J, Ek AC, Bjurulf P. Effects of dietary supplement on functional condition and clinical outcome measured with a modified Norton scale. Clinical Nutrition 1992;11(3):134-9.

\section{Leslie 2012 \{published data only\}}

Leslie WS, Woodward M, Lean MEJ, Theobald H, Watson L, Hankey CR. Improving the dietary intake of under nourished older people in residential care homes using an energy-enriching food approach: a cluster randomised controlled study. Journal of Human Nutrition and Dietetics 2012;26:387-394.

\section{Lin 2010 \{published data only\}}

Lin L-C, Huang Y-J, Su S-G, Watson R, Tsai BWJ, Wu S-C. Using spaced retrieval and Montessori-based activities in improving eating ability for residents with dementia. International Journal of Geriatric Psychiatry 2010;25(10):953-9.

\section{Lin 2011 \{published data only\}}

Lin L-C, Huang Y-J, Watson R, Wu S-C, Lee Y-C. Using a Montessori method to increase eating ability for institutionalised residents with dementia: a crossover design. Journal of Clinical Nursing 2011;20(21):3092.

\section{Mathey 2001a \{published data only\}}

Mathey MFAM, Vanneste VGG, De Graaf C, De Groot LCPGM, Van Staveren WA. Health effect of improved meal ambiance in a Dutch nursing home: A 1-year intervention study. Preventive Medicine 2001;32(5):416-23.

\section{Mathey 2001b \{published data only\}}

Mathey M-FAM, Siebelink Els, De Graaf C, Van Staveren WA. Flavor enhancement of food improves dietary intake and nutritional status of elderly nursing home residents. Journal of Gerontology: Medical Sciences 2001;56A(4):M200-M205.

Munk 2014 \{published data only\}

Munk T, Beck AM, Holst M, Rosenbom E, Rasmussen HH, Nielsen MA, et al. Positive effect of protein-supplemented hospital food on protein intake in patients at nutritional risk: a randomised controlled trial. Journal of Human Nutrition and Dietetics 2014;27:122-32.

Nijs 2006 \{published data only\}

* Nijs K, De Graaf C, Kok F. Effect of family style mealtimes on quality of life, physical performance, and body weight of nursing home residents: cluster randomised controlled trial. BMJ 2006;332(7551):1180-3.

Nijs KAND, De Graaf C, Siebelink E, Blauw YH, Vannest V, Kok FJ, et al. Effect of family-style meals on energy intake and risk of malnutrition in Dutch nursing home residents: a randomised controlled trial. Journal of Gerontology 2006;61A(9):935-42.

\section{Olofsson 2007 \{published data only\}}

Olofsson B, Stenvall M, Lundstrom M, Svensson O, Gustafson Y. Malnutrition in hip fracture patients: an intervention study. Journal of Clinical Nursing 2007;16(11):2027-38.

\section{Pivi 2011 \{published data only\}}

Pivi GAK, Da Silva RV, Juliano Y, Novo NF, Okamoto IH, Brant CQ, et al. A prospective study of nutrition education and oral nutritional supplementation in patients with Alzheimer's disease. Nutrition Journal 2011;10(1):98.

\section{Potter 2001 \{published data only\}}

* Potter JM, Roberts MA, McColl JH, Reilly JJ. Protein energy supplements in unwell elderly patients - a randomized controlled trial. JPEN Journal of Parenteral and Enteral Nutrition 2001;25(6):323-9.

Potter JM, Roberts MA, Reilly JJ, McColl JH. An evaluation of protein energy supplementation in medically ill admissions to a geriatric unit. Proceedings of the Nutrition Society 1998;3:88A.

Roberts M, Potter J, McColl J, Reilly J. Can prescription of sipfeed supplements increase energy intake in hospitalised older people with medical problems?. British Journal of Nutrition 2003;90(2):425-9.

\section{Remsburg 2001 \{published data only\}}

Remsburg RE, Luking A, Bara P, Radu C, Pineda D, Bennett RG, et al. Impact of a buffet-style dining program on weight and biochemical indicators of nutritional status in nursing home residents: a pilot study. Journal of the American Dietetic Association 2001;101(12):1460-3.

\section{Salva 2011 \{published data only\}}

Roqué M, Salvà A, Vellas B. Malnutrition in community-dwelling adults with dementia (NutriAlz Trial). Journal of Nutrition, Health \& Aging 2013;17(4):295-9. [PUBMED: 23538648] 
* Salva A, Andrieu S, Fernandez E, Schiffrin EJ, Moulin J, Decarli B, et al. Health and nutrition promotion program for patients with dementia (NutriAlz): cluster randomized trial. Journal of Nutrition Health \& Aging 2011;15(10):822-30.

Silver 2008 \{published data only\}

Silver HJ, Dietrich MS, Castellanos VH. Increased energy density of the home-delivered lunch meal improves 24-hour nutrient intakes in older adults. Journal of the American Dietetic Association 2008;108(12):2084-9.

\section{Simmons 2008 \{published data only\}}

Simmons SF, Keeler E, Zhuo X, Hickey KA, Sato H-W, Schnelle JF. Prevention of unintentional weight loss in nursing home residents: a controlled trial of feeding assistance. Journal of the American Geriatrics Society 2008;56(8):1466-73.

\section{Simmons 2010 \{published data only\}}

* Simmons SF, Zhuo X, Keeler E. Cost-effectiveness of nutrition interventions in nursing home residents: a pilot intervention. Journal of Nutrition Health \& Aging 2010;14(5):367-72.

\section{Smoliner 2008 \{published data only\}}

Smoliner C, Norman K, Scheufele R, Hartig W, Pirlich M, Lochs H. Effects of food fortification on nutritional and functional status in frail elderly nursing home residents at risk of malnutrition. Nutrition 2008;24(11-12):1139-44.

\section{Splett 2003 \{published data only\}}

Splett PL, Roth-Yousey LL, Vogelzang JL. Medical nutrition therapy for the prevention and treatment of unintentional weight loss in residential healthcare facilities. Journal of the American Dietetic Association 2003;103(3):352-62.

\section{Taylor 2006 \{published data only\}}

Taylor KA, Barr SI. Provision of small, frequent meals does not improve energy intake of elderly residents with dysphagia who live in an extended-care facility. Journal of the American Dietetic Association 2006;106(7):1115-8.

\section{Van den Berg 2015 \{published data only\}}

Van den Berg GH, Lindeboom R, Van der Zwet WC. The effects of the administration of oral nutritional supplementation with medication rounds on the achievement of nutritional goals: a randomised controlled trial. Clinical Nutrition (Edinburgh, Scotland) 2015;34:15-19.

\section{Van Ort 1995 \{published data only\}}

Van Ort S, Phillips LR. Nursing interventions to promote functional feeding. Journal of Gerontological Nursing 1995;10:6-14.

\section{References to studies excluded from this review}

Aleman-Mateo 2012 \{published data only\}

Aleman-Mateo H, Macias L, Esparza-Romero J, AstiazaranGarcia H, Blancas AL. Physiological effects beyond the significant gain in muscle mass in sarcopenic elderly men: evidence from a randomized clinical trial using a protein-rich food. Clinical Interventions in Aging 2012;7:225-34.
Allman 1990 \{published data only\}

Allman MA, Stewart PM, Tiller DJ, Horvath JS, Duggin GG, Truswell AS. Energy supplementation and the nutritional status of hemodialysis patients. American Journal of Clinical Nutrition 1990;51(4):558-62.

\section{Arias 2008 \{published data only\}}

Arias S, Bruzzone I, Blanco V, Inchausti M, Garcia F, Casavieja G, et al. Identification and early nutritional support in hospitalized malnourished patients. Nutrición Hospitalaria 2008;23(4):348-53.

\section{Asplund 2000 \{published data only\}}

Asplund K, Gustafson Y, Jacobsson C, Bucht G, Wahlin A, Peterson J, et al. Geriatric-based versus general wards for older acute medical patients: a randomized comparison of outcomes and use of resources. Journal of the American Geriatrics Society 2000;48(11):1381-8.

\section{Baldwin 2011 \{published data only\}}

Baldwin C, Spiro A, McGough C, Norman AR, Gillbanks A, Thomas $\mathrm{K}$, et al. Simple nutritional intervention in patients with advanced cancers of the gastrointestinal tract, non-small cell lung cancers or mesothelioma and weight loss receiving chemotherapy: a randomised controlled trial. Journal of Human Nutrition \& Dietetics 2011;24(5):431-40.

\section{Banerjee 1978 \{published data only\}}

Banerjee AK, Brocklehurst JC, Wainwright H, Swindell R. Nutritional status of long-stay geriatric in-patients: effects of a food supplement (Complan). Age and Ageing 1978;7(4):237-43.

\section{Bauer 2005 \{published data only\}}

Bauer J, Capra S, Battistutta D, Davidson W, Ash S, Cancer Cachexia Study G. Compliance with nutrition prescription improves outcomes in patients with unresectable pancreatic cancer. Clinical Nutrition 2005;24(6):998-1004.

\section{Beattie $\mathbf{2 0 0 0}$ \{published data only\}}

Beattie AH, Prach AT, Baxter JP, Pennington CR. A randomised controlled trial evaluating the use of enteral nutritional supplements postoperatively in malnourished surgical patients. Gut 2000;46(6):813-8.

\section{Beck 2008 \{published data only\}}

* Beck AM, Damkjaer K, Beyer N. Multifaceted nutritional intervention among nursing-home residents has a positive influence on nutrition and function. Nutrition 2008;24(11-12):1073-80.

Beck AM, Damkjaer K, Sorbye LW. Physical and social functional abilities seem to be maintained by a multifaceted randomized controlled nutritional intervention among old ( $>65$ years) Danish nursing home residents. Archives of Gerontology and Geriatrics 2010;50(3):351-5.

Beck AM, Damkjaer K, Tetens I. Lack of compliance of staff in an intervention study with focus on nutrition, exercise and oral care among old (65+ yrs) Danish nursing home residents. Aging Clinical and Experimental Research 2009;21(2):143-9. 


\section{Benati 2001 \{published data only\}}

Benati G, Delvecchio S, Cilla D, Pedone V. Impact on pressure ulcer healing of an arginine-enriched nutritional solution in patients with severe cognitive impairment. Archives of Gerontology and Geriatrics 2001;33(Supp 7):43-7.

Bonjour 2011 \{published data only\}

Bonjour JP, Benoit V, Pourchaire O, Rousseau B, Souberbielle JC. Nutritional approach for inhibiting bone resorption in institutionalized elderly women with vitamin $D$ insufficiency and high prevalence of fracture. Journal of Nutrition, Health \& Aging 2011;15(5):404-9.

\section{Bonjour 2012 \{published data only\}}

Bonjour JP, Benoit V, Rousseau B, Souberbielle JC. Consumption of vitamin D-and calcium-fortified soft white cheese lowers the biochemical marker of bone resorption TRAP $5 \mathrm{~b}$ in postmenopausal women at moderate risk of osteoporosis fracture. Journal of Nutrition 2012;142(4):698-703.

\section{Bonnefoy 2003 \{published data only\}}

Bonnefoy M, Cornu C, Normand S, Boutitie F, Bugnard F, Rahmani $A$, et al. The effects of exercise and protein-energy supplements on body composition and muscle function in frail elderly individuals: a long-term controlled randomised study. British Journal of Nutrition 2003;89(5):731-9.

\section{Bos 2001 \{published data only\}}

Bos C, Benamouzig R, Bruhat A, Roux C, Valensi P, Ferriere F, et al. Nutritional status after short-term dietary supplementation in hospitalized malnourished geriatric patients. Clinical Nutrition 2001;20(3):225-33.

\section{Botella-Carretero 2008 \{published data only\}}

Botella-Carretero JI, Iglesias B, Balsa JA, Zamarron I, Arrieta F, Vazquez C. Effects of oral nutritional supplements in normally nourished or mildly undernourished geriatric patients after surgery for hip fracture: a randomized clinical trial. JPEN Journal of Parenteral \& Enteral Nutrition 2008;32(2):120-8.

\section{Botella-Carretero 2010 \{published data only\}}

Botella-Carretero JI, Iglesias B, Balsa JA, Arrieta F, Zamarron I, Vazquez $C$. Perioperative oral nutritional supplements in normally or mildly undernourished geriatric patients submitted to surgery for hip fracture: a randomized clinical trial. Clinical Nutrition 2010;29(5):574-9.

\section{Boudville 2003 \{published data only\}}

Boudville N, Rangan A, Moody H. Oral nutritional supplementation increases caloric and protein intake in peritoneal dialysis patients. American Journal of Kidney Diseases 2003;41(3):658-63.

\section{Bunout 1989 \{published data only\}}

Bunout D, Aicardi V, Hirsch S, Petermann M, Kelly M, Silva G, et al. Nutritional support in hospitalized patients with alcoholic liver disease. European Journal of Clinical Nutrition 1989;43(9):615-21.
Bunout 2001 \{published data only\}

Bunout B, Barrera G, de la Maza P, Avendano M, Gattas V, Petermann M, et al. Effects of nutritional supplementation and resistance training on muscle strength in free living elders. Results of one year follow.. Journal of Nutrition, Health \& Aging 2004;8(2):68-75.

* Bunout D, Barrera G, de la Maza P, Avendano M, Gattas V, Petermann $\mathrm{M}$, et al. The impact of nutritional supplementation and resistance training on the health functioning of free-living Chilean elders: results of 18 months of follow-up. Journal of Nutrition 2001;131(9):2441S-6S.

\section{Carlsson 2011 \{published data only\}}

Carlsson M, Littbrand H, Gustafson Y, Lundin-Olsson L, Lindelof N, Rosendahl E, et al. Effects of high-intensity exercise and protein supplement on muscle mass in ADL dependent older people with and without malnutrition-A randomized controlled trial. Journal of Nutrition, Health \& Aging 2011;15(7):554-60.

\section{Carnaby 2006 \{published data only\}}

Carnaby G, Hankey GJ, Pizzi J. Behavioural intervention for dysphagia in acute stroke: a randomised controlled trial. Lancet Neurology 2006;5(1):31-7.

\section{Charlin 2002 \{published data only\}}

Charlin V, Carrasco F, Sepulveda C, Torres M, Kehr J. Nutritional supplementation according to energy and protein requirements in malnourished HIV-infected patients. Archivos Latinoamericanos de Nutricion 2002;52(3):267-73.

\section{Charras 2010 \{published data only\}}

Charras K, Fremontier M. Sharing meals with institutionalized people with dementia: a natural experiment. Journal of Gerontological Social Work 2010;53(5):436-48.

\section{Chernoff 1990 \{published data only\}}

Chernoff RS, Milton KY, Lipschitz DA. The effect of a very highprotein liquid formula on decubitus ulcers healing in long-term tube-fed institutionalized patients. Journal of the American Dietetic Association 1990;90:A-130.

Chin 2001 \{published data only\}

Chin APMJM, De Jong N, Schouten EG, Hiddink GJ, Kok FJ. Physical exercise and/or enriched foods for functional improvement in frail, independently living elderly: a randomized controlled trial. Archives of Physical Medicine and Rehabilitation 2001;82(6):811-7.

Collins 2005 \{published data only\} Collins CE, Kershaw J, Brockington S. Effect of nutritional supplements on wound healing in home-nursed elderly: a randomized trial. Nutrition 2005;21(2):147-55.

\section{Dangour 2011 \{published data only\}}

Dangour AD, Albala C, Adeo C, Elbourne D, Grundy E, Walker D, et al. A factorial-design cluster randomised controlled trial investigating the cost-effectiveness of a nutrition supplement and an exercise programme on pneumonia incidence, walking capacity and body mass index in older people living in 
Santiago, Chile: the CENEX study protocol. Nutrition Journal 2007;6(14):1-15.

* Dangour AD, Albala C, Allen E, Grundy E, Walker DG, Aedo C, et al. Effect of a nutrition supplement and physical activity program on pneumonia and walking capacity inChilean older people: a factorial cluster randomized trial. PLOS Medicine 2011;8(4):10.

\section{De Jong 1999 \{published data only\}}

De Jong N, Chin A Paw MJ, De Graaf C, Van Staveren WA. Effect of dietary supplements and physical exercise on sensory perception, appetite, dietary intake and body weight in frail elderly subjects. British Journal of Nutrition 2000;83(6):605-13.

* De Jong N, Chin A Paw MJ, De Groot LC, De Graaf C, Kok FJ, et al. Functional biochemical and nutrient indices in frail elderly people are partly affected by dietary supplements but not by exercise. Journal of Nutrition 1999;129(11):2028-36.

De Jong N, Chin A Paw MJ, De Groot LC, Hiddink GJ, Van Staveren WA. Dietary supplements and physical exercise affecting bone and body composition in frail elderly persons. American Journal of Public Health 2000;90(6):947-54.

\section{Delmi 1990 \{published data only\}}

Delmi M, Rapin CH, Bengoa JM, Delmas PD, Vasey H, Bonjour JP. Dietary supplementation in elderly patients with fractured neck of the femur. Lancet 1990;335(8696):1013-6.

\section{de Sousa 2012 \{published data only\}}

de Sousa OLV, Amaral TF. Three-week nutritional supplementation effect on long-term nutritional status of patients with mild Alzheimer disease. Alzheimer Disease and Associated Disorders 2012;26(2):119-23.

\section{Dhanraj 1997 \{published data only\}}

Dhanraj P, Chacko A, Mammen M, Bharathi R. Hospital-made diet versus commercial supplement in postburn nutritional support. Burns 1997;23(6):512-4.

\section{Dillabough 2011 \{published data only\}}

Dillabough A, Mammel J, Yee J. Improving nutritional intake in post-operative hip fracture patients: A quality improvement project. International Journal of Orthopaedic \& Trauma Nursing 2011;15(4):196-201.

\section{Edington 2004 \{published data only\}}

Edington J, Barnes R, Bryan F, Dupree E, Frost G, Hickson M, et al. A prospective randomised controlled trial of nutritional supplementation in malnourished elderly in the community: clinical and health economic outcomes. Clinical Nutrition 2004;23(2):195-204.

\section{Elkort 1981 \{published data only\}}

Elkort RJ, Baker FL, Vitale JJ, Cordano A. Long-term nutritional support as an adjunct to chemotherapy for breast cancer. JPEN Journal of Parenteral and Enteral Nutrition 1981;5(5):385-90.

\section{Endevelt 2011 \{published data only\}}

Endevelt R, Lemberger J, Bregman J, Kowen G, Berger-Fecht I, Lander $\mathrm{H}$, et al. Intensive dietary intervention by a dietitian as a case manager among community dwelling older adults: the edit study. Journal of Nutrition, Health and Aging 2011;15(8):624-30.

\section{Eneroth 2004 \{published data only\}}

Eneroth M, Larsson J, Oscarsson C, Apelqvist J. Nutritional supplementation for diabetic foot ulcers: the first RCT. Journal of Wound Care 2004;13(6):230-4.

\section{Espaulella 2000 \{published data only\}}

Espaulella J, Guyer H, Diaz-Escriu F, Mellado-Navas JA, Castells M, Pladevall M. Nutritional supplementation of elderly hip fracture patients. A randomized, double-blind, placebocontrolled trial. Age and Ageing 2000;29(5):425-31.

\section{Fiatarone 1994 \{published data only\}}

* Fiatarone MA, O'Neill EF, Ryan ND, Clements KM, Solares GR, Nelson ME, et al. Exercise training and nutritional supplementation for physical frailty in very elderly people. New England Journal of Medicine 1994;330(25):1769-75.

Fiatarone Singh MA, Bernstein MA, Ryan AD, O'Neill EF, Clements KM, Evans WJ. The effect of oral nutritional supplements on habitual dietary quality and quantity in frail elders. Journal of Nutrition, Health \& Aging 2000;4(1):5-12.

Forster 2005 \{published data only\}

* Forster SE, Gariballa SE, Powers HJ. Function-enabling diet (FED) study: a randomised, double-blind, placebo-controlled trial of the effects of energy, protein and micronutrient supplementation of hospitalised elderly patients. Proceedings of Nutrition Society 2005;64:25A.

Gariballa S, Forster S. Associations between underlying disease and nutritional status following acute illness in older people. Clinical Nutrition 2007;26(4):466-73.

Gariballa S, Forster S. Effects of dietary supplements on depressive symptoms in older patients: a randomised double-blind placebo-controlled trial. Clinical Nutrition 2007;26(5):545-51.

Gariballa S, Forster S, Walters S, Powers H. A randomized, double-blind, placebo-controlled trial of nutritional supplementation during acute illness. American Journal of Medicine 2006;119(8):693-9.

Gariballa S, Forster S. D 2007, 55(12):2030-4. Dietary supplementation and quality of life of older patients: a randomized, double-blind, placebo-controlled trial. Journal of the American Geriatrics Society 2007;55(12):2030-4.

\section{Gall 1998 \{published data only\}}

Gall MJ, Grimble GK, Reeve NJ, Thomas SJ. Effect of providing fortified meals and between-meal snacks on energy and protein intake of hospital patients. Clinical Nutrition 1998;17(6):259-64.

\section{Gariballa 1998 \{published data only\}}

Gariballa SE, Parker SG, Taub N, Castleden CM. A randomized, controlled, single-blind trial of nutritional supplementation after acute stroke. JPEN Journal of Parenteral and Enteral Nutrition 1998;22(5):315-9. 


\section{Gazzotti 2003 \{published data only\}}

Gazzotti C, Arnaud-Battandier F, Parello M, Farine S, Seidel L, Albert A, et al. Prevention of malnutrition in older people during and after hospitalisation: results from a randomised controlled clinical trial. Age and Ageing 2003;32(3):321-5.

\section{Gegerle 1986 \{published data only\}}

Gegerle P, Bengoa JM, Delmi M, Rapin CH, Loizeau E, Vasey H. Dietary survey on the effect of an oral nutritional supplement after femoral neck fracture [Enquete alimentaire apres fracture du col du femur: effet d'un supplement dietetique sur les apports nutritonnels]. Schweizerische Rundschau fur Medizin Praxis 1986;75(32):933-5.

\section{Gil Gregorio 2003 \{published data only\}}

Gil Gregorio P, Ramirez Diaz SP, Ribera Casado JM, Group D. Dementia and Nutrition. Intervention study in institutionalized patients with Alzheimer disease. Journal of Nutrition, Health \& Aging 2003;7(5):304-8.

\section{Goris 2003 \{published data only\}}

Goris AH, Vermeeren MA, Wouters EF, Schols AM, Westerterp KR. Energy balance in depleted ambulatory patients with chronic obstructive pulmonary disease: the effect of physical activity and oral nutritional supplementation. The British Journal of Nutrition 2003;89(5):725-31.

\section{Hogarth 1996 \{published data only\}}

Hogarth MB, Marshall P, Lovat LB, Palmer AJ, Frost CG, Fletcher $A E$, et al. Nutritional supplementation in elderly medical in-patients: a double-blind placebo-controlled trial. Age and Ageing 1996;25(6):453-7.

\section{Hopkinson 2010 \{published data only\}}

Hopkinson JB, Fenlon DR, Okamoto I, Wright DNM, Scott I, Addington-Hall JM, et al. The deliverability, acceptability, and perceived effect of the Macmillan approach to weight loss and eating difficulties: a phase II, cluster-randomized, exploratory trial of a psychosocial intervention for weight- and eatingrelated distress in people with advanced cancer. Journal of Pain and Symptom Management 2010;40(5):684-95.

\section{Houles 2010 \{published data only\}}

Houles M. Randomised study of a multi-disciplinary programme focusing on geriatric syndromes in vulnerable, elderly individuals living at home (Dutch EASYcare Study). Cahiers de l'Annee Gerontologique 2010;2(3):166-8.

\section{Hubbard 2008 \{published data only\}}

Hubbard GP, Bolch R, Holdoway H, Beams A, Kerr A, Robertson $D$, et al. $A$ randomised, controlled trial of the effects of an energy-dense supplement on energy intake, appetite and blood lipids in malnourished community-based elderly patients. Journal of Human Nutrition and Dietetics 2008;21(4):390-1.

\section{Hubsch 1992 \{published data only\}}

Hubsch S, Volkert D, Oster P, Schlierf G. Effect of dietary supplementation on weight and protein status in undernourished geriatric patients. Clinical Nutrition 1992;11:97.

\section{Huisman 2012 \{published data only\}}

Huisman EJ, Van Hoek B, Van Soest H, Van Nieuwkerk KM, Arends JE, Siersema PD, et al. Preventive versus "on-demand" nutritional support during antiviral treatment for hepatitis C: a randomized controlled trial. Journal of Hepatology 2012;57(5):1069-75.

\section{Isenring 2003 \{published data only\}}

Isenring E, Capra S, Bauer J, Davies PSW. The impact of nutrition support on body composition in cancer outpatients receiving radiotherapy. Acta Diabetologica 2003;40(SUPPL. 1):S162-S4.

\section{Isenring 2004 \{published data only\}}

Isenring E, Capra S, Bauer J. Patient satisfaction is rated higher by radiation oncology outpatients receiving nutrition intervention compared with usual care. Journal of Human Nutrition and Dietetics 2004;17(2):145-52.

\section{Jahnavi 2010 \{published data only\}}

Jahnavi G, Sudha $\mathrm{CH}$. Randomised controlled trial of food supplements in patients with newly diagnosed tuberculosis and wasting. Singapore Medical Journal 2010;51(12):957-62.

James 2006 \{published data only\} James M, Stokes E, Bowling T, Smedley F, Jones P, Silk D. An economic assessment of peri-operative oral nutritional supplements in patients undergoing elective moderate to major lower gastrointestinal surgery. Proceedings of the Nutrition Society 2006;65:4A.

\section{Johnson 1993 \{published data only\}} Johnson LE, Dooley PA, Gleick JB. Oral nutritional supplement use in elderly nursing home patients. Journal of the American Geriatrics Society 1993;41(9):947-52.

Keele 1997 \{published data only\}

Keele AM, Bray MJ, Emery PW, Duncan HD, Silk DB. Two phase randomised controlled clinical trial of postoperative oral dietary supplements in surgical patients. Gut 1997;40(3):393-9.

\section{Kikutani 2006 \{published data only\}}

Kikutani T, Enomoto R, Tamura F, Oyaizu K, Suzuki A, Inaba S. Effects of oral functional training for nutritional improvement in Japanese older people requiring long-term care. Gerodontology 2006;23(2):93-8.

\section{Knowles 1988 \{published data only\}}

Knowles JB, Fairbarn MS, Wiggs BJ, Chan-Yan C, Pardy RL. Dietary supplementation and respiratory muscle performance in patients with COPD. Chest 1988;93(5):977-83.

\section{Krondl 1999 \{published data only\}}

Krondl M, Coleman PH, Bradley CL, Lau D, Ryan N. Subjectively healthy elderly consuming a liquid nutrition supplement maintained body mass index and improved some nutritional parameters and perceived well-being. Journal of the American Dietetic Association 1999;99(12):1542-8. 
Kruizenga 2004 \{published data only\}

Kruizenga HM, Seidell J, Ader HJ, Van Bokhorst MAE. Early screening and treatment of malnutrition in hospital reduces length of stay. Clinical Nutrition 2004;23(764):04-A-228.

\section{KuhImann 1997 \{published data only\}}

Kuhlmann MK, Schmidt F, Kohler H. Oral nutritional support in malnourished HD-patients. Preliminary results of a randomised controlled study. Journal of the American Society of Nephrology 1997;8(SS):A0924.

\section{Kwok 2001 \{published data only}

Kwok T, Woo J, Kwan M. Does low lactose milk powder improve the nutritional intake and nutritional status of frail older Chinese people living in nursing homes?. Journal of Nutrition, Health \& Aging 2001;5(1):17-21.

\section{Kwok 2012 \{published data only}

Kwok TCY, Lam LCW, Sea MMM, Goggins W, Woo J. A randomized controlled trial of dietetic interventions to prevent cognitive decline in old age hostel residents. European Journal of Clinical Nutrition 2012;66(10):1135-40.

\section{Lauque 2000 \{published data only\}}

Lauque S, Arnaud-Battandier F, Mansourian R, Guigoz Y, Paintin M, Nourhashemi F, et al. Protein-energy oral supplementation in malnourished nursing-home residents. A controlled trial. Age and Ageing 2000;29(1):51-6.

\section{Lauque 2004 \{published data only\}}

Lauque S, Arnaud-Battandier F, Gillette S, Plaze J-M, Andrieu S, Cantet $C$, et al. Improvement of weight and fat-free mass with oral nutritional supplementation in patients with Alzheimer's disease at risk of malnutrition: a prospective randomized study. Journal of the American Geriatrics Society 2004;52(10):1702-7.

\section{Lawson 2000 \{published data only\}}

Lawson RM, Doshi MK, Ingoe LE, Colligan JM, Barton JR, Cobden I. Compliance of orthopaedic patients with postoperative oral nutritional supplementation. Clinical Nutrition 2000;19(3):171-5.

\section{Le Cornu 2000 \{published data only\}}

Le Cornu KA, McKiernan FJ, Kapadia SA, Neuberger JM. A prospective randomized study of preoperative nutritional supplementation in patients awaiting elective orthotopic liver transplantation. Transplantation 2000;69(7):1364-9.

\section{Lee 2013 \{published data only\}}

Lee L-C, Tsai AC, Want J-Y, Hurng B-S, Hsu J-C, Tsai H-J. Needbased intervention is an effective strategy for improving the nutritional status of older people living in a nursing home: a randomized controlled trial. International Journal of Nursing Studies 2013;50:1580-8

\section{Leon 2001 \{published data only\}}

Leon JB, Majerle AD, Soinski JA, Kushner I, Ohri-Vachaspati P, Sehgal AR. Can a nutrition intervention improve albumin levels among hemodialysis patients? A pilot study. Journal of Renal Nutrition 2001;11(1):9-15.

\section{Leon 2006 \{published data only\}}

Leon JB, Albert JM, Gilchrist G, Kushner I, Lerner E, Mach S, et al. Improving albumin levels among hemodialysis patients: a community-based randomized controlled trial. American Journal of Kidney Diseases 2006;48(1):28-36.

Locher 2011 \{published data only\}

Locher JL, Bales CW, Ellis AC, Lawrence JC, Newton L, Ritchie CS, et al. A theoretically based behavioral nutrition intervention for community elders at high risk: The B-NICE randomized controlled clinical trial. Journal of Nutrition in Gerontology and Geriatrics 2011;30(4):384-402.

MacFie 2000 \{published data only\}

MacFie J, Woodcock NP, Palmer MD, Walker A, Townsend S, Mitchell CJ. Oral dietary supplements in pre- and postoperative surgical patients: a prospective and randomized clinical trial. Nutrition 2000;16(9):723-8.

\section{Mamhidir 2007 \{published data only\}}

Mamhidir A, Karlsson I, Norberg A. Weight increase in patients with dementia, and alteration in meal routines and meal environment after integrity promoting care. Journal of Clinical Nursing 2007;16(5):987-96.

\section{Manders 2006 \{published data only\}}

* Manders M. Nutritional care in old age: the effect of supplementation on nutritional status and performance [PhD thesis]. Wageningen: Wageningen University, 2006.

Manders M, De Groot CPGM, Blauw YH, Dhonukshe-Rutten RAM, Van Hoeckel-Prust L, Bindels JG, et al. Effect of a nutrientenriched drink on dietary intake and nutritional status in institutionalised elderly. European Journal of Clinical Nutrition 2009;63(10):1241-50.

Manders M, De Groot LCPGM, Hoefnagels WHL, DhonuksheRutten RAM, Wouters-Wesseling W, Mulders AJMJ, et al. The effect of a nutrient dense drink on mental and physical function in institutionalized elderly people. Journal of Nutrition, Health \& Aging 2009;13(9):760-7.

\section{McEvoy 1982 \{published data only\}}

McEvoy AW, James OF. The effect of a dietary supplement (Build-up) on nutritional status in hospitalized elderly patients. Human Nutrition Applied Nutrition 1982;36(5):374-6.

\section{McMurdo 2009 \{published data only\}}

McMurdo MET, Price RJG, Shields M, Potter J, Stott DJ. Should oral nutritional supplementation be given to undernourished older people upon hospital discharge? A controlled trial. Journal of the American Geriatrics Society 2009;57(12):2239-45.

\section{Moretti 2009 \{published data only\}}

Moretti HD, Johnson AM, Keeling-Hathaway TJ. Effects of protein supplementation in chronic hemodialysis and peritoneal dialysis patients. Journal of Renal Nutrition 2009;19(4):298-303. 
Navrátilová 2007 \{published data only\}

Navrátilová M, Jarkovský J, Cešková E, Leonard B, Sobotka L. Alzheimer disease: malnutrition and nutritional support. Clinical and Experimental Pharmacology and Physiology 2007;34:S11-S3

\section{Nayel 1992 \{published data only\}}

Nayel H, el-Ghoneimy E, el-Haddad S. Impact of nutritional supplementation on treatment delay and morbidity in patients with head and neck tumors treated with irradiation. Nutrition 1992;8(1):13-8.

\section{Ödlund Olin 2003 \{published data only\}}

Ödlund Olin A, Armyr I, Soop M, Jerström S, Classon I, Cederholm T, et al. Energy-dense meals improve energy intake in elderly residents in a nursing home. Clinical Nutrition (Edinburgh, Scotland) 2003;22(2):125-31.

\section{Olin 1996 \{published data only\}}

Olin AO, Osterberg P, Hadell K, Armyr I, Jerstrom S, Ljungqvist O. Energy-enriched hospital food to improve energy intake in elderly patients. JPEN Journal Parenteral \& Enteral Nutrition 1996;20(2):93-7.

\section{Otte 1989 \{published data only\}}

Otte KE, Ahlburg P, D'Amore F, Stellfeld M. Nutritional repletion in malnourished patients with emphysema. JPEN Journal Parenteral \& Enteral Nutrition 1989;13(2):152-6.

Payette 2002 \{published data only\}

Payette H, Boutier V, Coulombe C. Efficacy of nutritional intervention in the free-living frail elderly. American Journal of Clinical Nutrition 2002;75(2):340S.

\section{Price 2005 \{published data only\}}

Price R, Daly F, Pennington CR, McMurdo MET. Nutritional supplementation of very old people at hospital discharge increases muscle strength: a randomised controlled trial. Gerontology 2005;51(3):179-85.

\section{Rana 1992 \{published data only\}}

Rana SK, Bray J, Menzies-Gow N, Jameson J, Payne James JJ, Frost $P$, et al. Short term benefits of post-operative oral dietary supplements in surgical patients. Clinical Nutrition 1992;11(6):337-44

\section{Richeson \& Neil 2004 \{published data only\}}

Richeson NE, Neil DJ. Therapeutic recreation music intervention to decrease mealtime agitation and increase food intake in older adults with dementia. American Journal of Recreation Therapy 2004;3(1):37-41.

\section{Roberts 2013 \{published data only\}}

Roberts HC, Pilgrim AL, Elia M, Jackson AA, Cooper C, Sayer AA, et al. Southampton Mealtime Assistance Study: design and methods. BMC Geriatrics 2013;13:5.

\section{Robinson 2002 \{published data only\}}

Robinson S, Clump D, Weitzel T, Henderson L, Lee K, Schwartz C, et al. The Memorial Meal Mates: a program to improve nutrition in hospitalized older adults. Geriatric Nursing (New York, NY) 2002;23(6):332-5.

Rosendahl 2006 \{published data only\}

Rosendahl E, Lindelof N, Littbrand H, Yifter-Lindgren E, Lundin-Olsson L, Haglin L, et al. High-intensity functional exercise program and protein-enriched energy supplement for older persons dependent in activities of daily living: a randomised controlled trial. Australian Journal of Physiotherapy 2006;52(2):105-13.

\section{Roy 2006 \{published data only\}}

Roy MA, Payette H. Meals-on-wheels improves energy and nutrient intake in a frail free-living elderly population. Journal of Nutrition, Health \& Aging 2006;10(6):554-60

Rypkema 2004 \{published data only\}

Rypkema G, Adang E, Dicke H, Naber T, de Swart B, Disselhorst $\mathrm{L}$, et al. Cost-effectiveness of an interdisciplinary intervention in geriatric inpatients to prevent malnutrition. Journal of Nutrition, Health \& Aging 2004;8(2):122-7.

Saudny-Unterberger 1997 \{published data only\}

Saudny-Unterberger H, Martin JG, Gray-Donald K. Impact of nutritional support on functional status during an acute exacerbation of chronic obstructive pulmonary disease. American Journal of Respiratory and Critical Care Medicine 1997;156(3 Pt 1):794-9.

Shinnar 1983 \{published data only\}

Shinnar SE. Use of adaptive equipment in feeding the elderly. Journal of the American Dietetic Association 1983;83(3):321-2.

\section{Simmons 2004 \{published data only\}}

Simmons SF, Schnelle JF. Individualized feeding assistance care for nursing home residents: staffing requirements to implement two interventions. Journals of Gerontology - Series A Biological Sciences and Medical Sciences 2004;59(9):966-73.

\section{Smedley 2004 \{published data only\}}

Smedley F, Bowling T, James M, Stokes E, Goodger C, O'Connor $\mathrm{O}$, et al. Randomized clinical trial of the effects of preoperative and postoperative oral nutritional supplements on clinical course and cost of care. British Journal of Surgery 2004;91(8):983-90.

\section{Somanchi 2011 \{published data only\}}

Somanchi M, Tao X, Mullin GE. The facilitated early enteral and dietary management effectiveness trial in hospitalized patients with malnutrition. JPEN. Journal of Parenteral and Enteral Nutrition 2011;35(2):209-16.

\section{Soneff 1994 \{published data only\}}

Soneff R, McGeachy F, Davison K, McCargar L, Therien G. Effectiveness of two training methods to improve the quality of food service in small facilities for adult care. Journal of the American Dietetic Association 1994;94(8):869-73.

\section{Southgate 2010 \{published data only\}}

Southgate KM, Keller HH, Reimer HD. Determining knowledge and behaviour change after nutrition screening among older 
adults. Canadian Journal of Dietetic Practice and Research 2010;71(3):128-33.

\section{Starke 2011 \{published data only\}}

Starke J, Schneider H, Alteheld B, Stehle P, Meier R. Short-term individual nutritional care as part of routine clinical setting improves outcome and quality of life in malnourished medical patients. Clinical Nutrition 2011;30(2):194-201.

\section{Stauffer 1986 \{published data only\}}

Stauffer JL, Carbone JE, Bendoski MT. Effects of diet supplementation on anthropometric and laboratory nutritional parameters in malnourished ambulatory patients with severe Chronic Obstructive Pulmonary disease (COPD). American Review of Respiratory Disease 1986;133:A204.

Steiner 2003 \{published data only\}

Steiner MC, Barton RL, Singh SJ, Morgan MD. Nutritional enhancement of exercise performance in chronic obstructive pulmonary disease: a randomised controlled trial. Thorax 2003;58(9):745-51.

\section{Stotts 2009 \{published data only\}}

Stotts NA, Hopf HW, Kayser-Jones J, Chertow GM, Cooper BA, $\mathrm{Wu} \mathrm{H}$. Increased fluid intake does not augment capacity to lay down new collagen in nursing home residents at risk for pressure ulcers: a randomized, controlled clinical trial. Wound Repair and Regeneration 2009;17(6):780-8.

\section{Teixido-Planas 2005 \{published data only\}}

Teixido-Planas J, Ortiz A, Coronel F, Montenegro J, LopezMenchero R, Ortiz R, et al. Oral protein-energy supplements in peritoneal dialysis: a multicenter study. Peritoneal Dialysis International 2005;25(2):163-72.

\section{Tkatch 1992 \{published data only\}}

Tkatch L, Rapin CH, Rizzoli R, Slosman D, Nydegger V, Vasey H, et al. Benefits of oral protein supplementation in elderly patients with fracture of the proximal femur. Journal of the American College of Nutrition 1992;11(5):519-25.

\section{Vetter 1992 \{published data only\}}

Vetter NJ, Lewis PA, Ford D. Can health visitors prevent fractures in elderly people?. BMJ 1992;304(6831):888-90.

\section{Vlaming 2001 \{published data only\}}

Vlaming S, Biehler A, Hennessey EM, Jamieson CP, Chattophadhyay S, Obeid OA, et al. Should the food intake of patients admitted to acute hospital services be routinely supplemented? A randomized placebo controlled trial. Clinical Nutrition 2001;20(6):517-26.

\section{Watanabe 2010 \{published data only\}}

Watanabe S, Kumagai S, Shibata H. An interventional trial to improve nutritional status in the community-dwelling elderly. Nippon Ronen Igakkai Zasshi 2010;47(5):422-5.

\section{Williams 1989 \{published data only\}}

Williams CM, Driver LT, Older J, Dickerson JW. A controlled trial of sip-feed supplements in elderly orthopaedic patients. European Journal of Clinical Nutrition 1989;43(4):267-74.
Wong 2010 \{published data only\}

Wong SS, O'Driscoll J, Weldon M, Yau CY. Do regular oral nutritional supplements improve clinical outcomes in patients with Clostridium difficile infection: a pilot study. Proceedings of the Nutrition Society 2010;69(OCE2):E174.

Woo 1994 \{published data only\}

Woo J, Ho SC, Mak YT, Law LK, Cheung A. Nutritional status of elderly patients during recovery from chest infection and the role of nutritional supplementation assessed by a prospective randomized single-blind trial. Age and Ageing 1994;23(1):40-8.

\section{Wouters-Wesseling 2002 \{published data only\}}

Wouters-Wesseling W, Rozendaal M, Snijder M, Graus Y, Rimmelzwaan G, De Groot L, et al. Effect of a complete nutritional supplement on antibody response to influenza vaccine in elderly people. Journals of Gerontology Series $A$ : Biological Sciences \& Medical Sciences 2002;57(9):M563-6.

\section{Wright 2006 \{published data only\}}

Wright L, Hickson M, Frost G. Eating together is important: using a dining room in an acute elderly medical ward increases energy intake. Journal of Human Nutiriton \& Dietetics 2006;19(1):23-6.

\section{WY Lin 2010 \{published data only\}}

Lin WY, Huang HY, Liu CS, Li Cl, Lee SD, Lin CC, et al. A hospitalbased multidisciplinary approach improves nutritional status of the elderly living in long-term care facilities in middle Taiwan. Archives of Gerontology and Geriatrics 2010;50 Suppl 1:S22-6.

\section{Yamaguchi 1998 \{published data only\}}

Yamaguchi LY, Coulston AM, Lu NC, Dixon LB, Craig LD. Improvement in nutrient ilntake by elderly meals-on-wheels participants receiving a liquid nutrition supplement. Nutrition Today 1998;33(1):37.

\section{Young 2004 \{published data only\}}

Parrott MD, Young KWH, Greenwood CE. Energy-containing nutritional supplements can affect usual energy intake post supplementation in institutionalized seniors with probable Alzheimer's disease. Journal of the American Geriatrics Society 2006;54(9):1382-7.

Young KWH, Greenwood CE, Van Reekum R, Binns MA. A randomized, crossover trial of high-carbohydrate foods in nursing home residents with Alzheimer's disease: associations among intervention response, body mass index, and behavioral and cognitive function.. Journal of Gerontology: Medical Sciences 2005;60(8):1039-45.

* Young KWH, Greenwood CE, Van Reekum R, Binns MA. Providing nutrition supplements to institutionalized seniors with probable Alzheimer's disease is least beneficial to those with low body weight status. Journal of the American Geriatrics Society 2004;52(8):1305-12. 


\section{References to studies awaiting assessment}

\section{Allen 2014 \{published data only\}}

Allen VJ, Methven L, Gosney M. Impact of serving method on the consumption of nutritional supplement drinks: randomized trial in older adults with cognitive impairment. Journal of Advanced Nursing 2014;70(6):1323-33.

\section{Borges 2003 \{published data only\}}

Borges VC. [Repercussão Nutricional nas Doenças Neurológicas da Velhice: Alzheimer e Parkinson]. [texto na internet]. Nutrição Total SãoPaulo. Disponível em; [http://www.nutritotal.com.br] 2003.

\section{Burns 1998 \{published data only\}}

Burns BL, Davis EMC. [Cuidado Nutricional nas Doenças do Sistema Nervoso]. In: Mahan LK, Stump SE. Alimentos, Nutrição e Dietoterapia. 9edition. São Paulo: Roca. 1998:896-7.

\section{Deutz 2016 \{published data only\}}

Deutz NE, Matheson EM, Matarese LE, Luo M, Baggs GE, Nelson Jl, et al. Readmission and mortality in malnourished, older, hospitalized adults treated with a specialized oral nutritional supplement: a randomized clinical trial. Clinical Nutrition (Edinburgh, Scotland) 2016;35(1):18-26.

\section{Ekinci 2016 \{published data only\}}

Ekinci O, Yanik S, Terzioglu Bebitoglu B, Yilmaz Akyuz E, Dokuyucu A, Erdem S. Effect of calcium beta-hydroxybeta-methylbutyrate (CaHMB), vitamin D, and protein supplementation on postoperative immobilization in malnourished older adult patients with hip fracture: a randomized controlled study. Nutrition in Clinical Practice 2016 [Epub ahead of print]; Vol. 10. [DOI: 10.1177/0884533616629628]

ISRCTN04327195 \{unpublished data only\}

\section{ISRCTN96923961 \{unpublished data only\}}

\author{
Jobse 2015 \{published data only\} \\ Jobse I, Liao Y, Bartram M, Delantonio K, Uter W, Stehle P, et \\ al. Compliance of nursing home residents with a nutrient- \\ and energy-dense oral nutritional supplement determines \\ effects on nutritional status. Journal of Nutrition, Health \& Aging \\ 2015;19(3):356-64.
}

\section{Lee 2015 \{published data only\}}

Lee Lc, Tsai Ac, Wang Jy. Need-based nutritional intervention is effective in improving handgrip strength and Barthel Index scores of older people living in a nursing home: a randomized controlled trial. International Journal of Nursing Studies 2015;52(5):904-12.

\section{Leslie 2013 \{published data only\}}

Leslie WS, Woodward M, Lean MEJ, Theobald H, Watson L, Hankey CR. Improving the dietary intake of under nourished older people in residential care homes using an energyenriching food approach: a cluster randomised controlled study. Journal of Human Nutrition and Dietetics 2013;26:387-94.

\section{Luna-Ramos 2016 \{published data only\}}

Luna-Ramos GK, Pedraza-Zarate MA, Franco-Alvarez N, Gonzalez-Velazquez F. Diet and polymer standard vs. standard in the nutritional status of elderly patients with fragility. Revista Medica del Instituto Mexicano del Seguro Social 2016;54(4):439-45.

\section{Madigan 1994 \{published data only\}}

Madigan C. Benefits of dietary supplementation in elderly patients with fractured neck of femur [MSc dissertation]. Sydney: University of Sydney, 1994.

\section{Moore 2010 \{published data only\}}

Moore JR. Familiar physical activity to familiar music: The effects on apathy, agitation, eating ability, and dietary intake in institutionalised older adults with dementia [Dissertation]. Amherst: University of Massachusetts, 2010.

\section{Parsons 2016 \{published data only\}}

Parsons EL, Stratton RJ, Cawood AL, Smith TR, Elia M. Oral nutritional supplements in a randomised trial are more effective than dietary advice at improving quality of life in malnourished care home residents. Clinical Nutrition 2016 [Epub ahead of print]; Vol. 11. [DOI: 10.1016/j.clnu.2016.01.002]

\section{Pouyssegur 2015 \{published data only\}}

Pouyssegur V, Brocker P, Schneider SM, Philip JL, Barat P, Reichert $E$, et al. An innovative solid oral nutritional supplement to fight weight loss and anorexia: open randomised controlled trial of efficacy in institutionalised, malnourished older adults. Age and Ageing 2015;44:245-51.

\section{Scorer 1990 \{published data only\}}

Scorer HJM. The effect of nutritional supplementation with Ensure on the general health of undernourished elderly patients in the community. Abbott UK Medical, 1990.

\section{Simmons 2013 \{published data only\}}

Simmons SF, Durkin DW, Shotwell MS, Erwin S, Schnelle JF. A staff training and management intervention in VA long-term care: impact on feeding assistance care quality. Translational Behavioral Medicine 2013;13:189-99.

\section{Simmons 2015 \{published data only\}}

Simmons SF, Keeler E, An R, Liu X, Shotwell MS, Kuertz B, et al. Cost-effectiveness of nutrition intervention in long-term care. Journal of the American Geriatrics Society 2015; Vol. 63, issue 11:2308-16

\section{Stelten 2015 \{published data only\}}

Stelten S, Dekker IM, Ronday EM, Thijs A, Boelsma E, Peppelenbos, et al. Protein-enriched 'regular products' and their effect on protein intake in acute hospitalized older adults: a randomised controlled trial. Clinical Nutrition 2015;34:409-14.

\section{Stow 2015 \{published data only\}}

Stow R, Ives N, Smith C, Rick C, Rushton A. A cluster randomised feasibility trial evaluating nutritional interventions in the treatment of malnutrition in care home adult residents. Trials 2015; 16:433. 
Sutton 2006 \{published data only\}

Sutton D, Hubbard GP, Bolch R, Cawood AL, Stevens L, Bircher $\mathrm{G}$, et al. Evaluation of a renal-specific oral nutritional supplement in the management of chronic renal failure in haemodialysis and peritoneal dialysis patients. Journal of Renal Care 2006;32:Abstracts: 34

Turano 1999 \{published data only\}

Turano W, Almeida CCC. [Educação Nutricional]. In: Gouveia ELC. Nutrição Saúde e Comunidade. 2 edition. Rio de Janeiro: Revinter 1999:57-60.

\section{White 1999 \{published data only\}}

White JV. In: The role of nutrition in chronic disease. Nutrition Screening Initiative. A project of American Academy of Family Physicians. The American Dietetic Association and National Council on the Ageing, USA 1997.

Zhong 2016 \{published data only\}

Zhong Y, Cohen JT, Goates S, Luo M, Nelson J, Neumann PJ. The cost-effectiveness of oral nutrition supplementation for malnourished older hospital patients. Applied Health Economics \& Health Policy 2016 [Epub ahead of print]; Vol. 4.

\section{Additional references}

\section{Agarwal 2011}

Agarwal E, Ferguson M, Banks M, Bauer J, Capra S, Isenring E. Nutritional status and dietary intake of acute care patients: results from the Nutrition Care Day Survey 2010. Clinical Nutrition 2012;31(1):41-7.

\section{Allison 2000}

Allison SP. Malnutrition, disease, and outcome. Nutrition 2000;16(7-8):590-3.

\section{Avenell 2001}

Avenell A, Handoll HHG, Grant AM. Lessons for search strategies from a systematic review, in the Cochrane Library, of nutritional supplementation trials in patients after hip fracture. The American Journal of Clinical Nutrition 2001;73(3):505-10.

\section{BAPEN 2009}

British Association for Parenteral and Enteral Nutrition (BAPEN). Combatting Malnutrition: Recommendations for Action. Redditch: BAPEN, 2009.

\section{BAPEN 2012}

BAPEN Quality Group. Malnutrition Matters - Meeting Quality Standards in Nutritional Care: A Toolkit for Commissioners and Providers in England. Redditch, Worcestershire: BAPEN Quality Group, 2012

\section{Bistrian 1974}

Bistrian BR, Blackburn GL, Hallowell E, Heddle R. Protein status of general surgical patients. Journal of the American Medical Association 1974;230(6):858-60.

\section{Butterworth 1974}

Butterworth CEJ. The skeleton in the hospital closet. Nutrition Today 1974;9(2):4-8.

\section{Chima 1997}

Chima CS, Barco K, Dewitt MLA, Maeda M, Teran JC, Mullen KD. Relationship of nutritional status to length of stay, hospital costs, and discharge status of patients hospitalized in the medicine service. Journal of the American Dietetic Association 1997;97(9):975-8.

\section{COE 2003}

Council of Europe (COE). Food and nutritional care in hospitals: how to prevent undernutrition. Nutrition programmes in Hospitals Group for the Committee of experts on Nutrition, Food \& Safety and Consumer Health. Strasbourg: Council of Europe, 2003.

\section{Cole 2012}

Cole D. Optimising nutrition for older people with dementia. Nursing Standard 2012;26(20):41-8.

\section{Corish 2000a}

Corish CA, Kennedy NP. Protein-energy undernutrition in hospital in-patients. British Journal of Nutrition 2000;83(6):575-91.

Corish 2000b

Corish CA, Flood P, Mulligan S, Kennedy NP. Apparent low frequency of undernutrition in Dublin hospital in-patients: should we review the anthropometric thresholds for clinical practice?. British Journal of Nutrition 2000;84(3):325-35.

\section{Deeks 2011}

Deeks JJ, Higgins JPT, Altman DG (editors). Chapter 9: Analysing data and undertaking meta-analyses. In: Higgins JPT, Green $S$ (editors). Cochrane Handbook for Systematic Reviews of Interventions Version 5.1.0 (updated March 2011). The Cochrane Collaboration, 2011. Available from handbook.cochrane.org.

\section{DOH 2003}

Department of Health (DOH). The essence of care: patient focused benchmarks for clinical governance. London, UK: Department of Health, 2003.

DOH 2007

Department of Health (DOH). Improving nutrition care: a joint action plan from the Department of Health and the Nutrition Summit Stakeholders. London, UK: Department of Health, 2007.

\section{Donini 2003}

Donini LM, Savina C, Cannella C. Eating habits and appetite control in the elderly: the anorexia of aging. International Psychogeriatrics 2003;15(1):73-87.

\section{Edington 2000}

Edington J, Boorman J, Durrant ER, Perkins A, Giffin CV, James $R$, et al. Prevalence of malnutrition on admission to four hospitals in England. Clinical Nutrition 2000;19(3):191-5. 


\section{Elia 2003}

Elia M. The "MUST" report. Nutritional screening of adults: a multidisciplinary responsibility. Redditch, Worcs, UK: Malnutrition Advisory Group of the British Association for Parenteral and Enteral Nutrition, 2003.

\section{Elia 2009}

Elia M, Russell CA. British Associaton for Parenteral, Enteral Nutrition (BAPEN). Nutrition screening survey in the UK in 2008. Hospitals, care homes and mental health units. www.bapen.org.uk (accessed 16 March 2012).

\section{EuroQol group 1990}

EuroQol. EuroQol-a new facility for the measurement of health related quality of life. Health Policy 1990;16(3):199-208.

\section{Gallagher 1996}

Gallagher Allred CR, Coble Voss A, Finn SC, McCamish MA Malnutrition and clinical outcomes: the case for medical nutrition therapy. Journal of the American Dietetic Association 1996;96(4):361-9.

\section{Gilson 1975}

Gilson BS, Gilson JS, Bergner M, Bobbitt RA, Kressel S, Pollard WE, et al. The sickness impact profile: development of an outcome measure of healthcare. American Journal of Public Health 1975;65:1304.

\section{Green 2006}

Green S, Watson R. Nutritional screening and assessment tools for older adults: literature review. Journal of Advanced Nursing 2006;54:477-90.

\section{Higgins 2002}

Higgins JPT, Thompson SG. Quantifying heterogeneity in a meta-analysis. Statistics in Medicine 2002;21:1539-58.

\section{Higgins 2003}

Higgins JPT, Thompson SG, Deeks JJ, Altman DG. Measuring inconsistency in meta-analysis. BMJ 2003;327:557-60.

\section{Higgins 2011a}

Higgins JPT, Altman DG, Sterne JAC (editors). Chapter 8: Assessing risk of bias in included studies. In: Higgins JPT, Green $\mathrm{S}$ (editors). Cochrane Handbook for Systematic Reviews of Interventions Version 5.1.0 (updated March 2011). The Cochrane Collaboration, 2011. Available from handbook.cochrane.org.

\section{Higgins 2011b}

Higgins JPT, Altman DG, Gøtzsche PC, Jüni P, Moher D, Oxman AD, et al. The Cochrane Collaboration's tool for assessing risk of bias in randomised trials. BMJ 2011;343:d5928.

\section{Higgins 2011c}

Higgins JPT, Deeks JJ, Altman DG (editors). Chapter 16: Special topics in statistics. In: Higgins JPT, Green S (editors), Cochrane Handbook for Systematic Reviews of Interventions Version 5.1.0 (updated March 2011). The Cochrane Collaboration, 2011. Available from handbook.cochrane.org.

\section{Hill 1977}

Hill GL, Blackett RL, Pickford I, Burkinshaw L, Young GA, Warren JV, et al. Malnutrition in surgical patients. An unrecognised problem. Lancet 1977;1:689-92.

\section{Jeejeebhoy 1986}

Jeejeebhoy KN. Muscle function and nutrition. Gut 1986;27(Suppl 1):25-39.

\section{Jefferies 2011}

Jefferies D, Johnson M, Ravens J. Nurturing and nourishing: the nurses' role in nutritional care. Journal of Clinical Nursing 2011;20(3-4):317-30

\section{Jensen 2010}

Jensen GL, Mirtallo J, Compher C, Dhaliwal R, Forbes A, Grijalba RF, et al. Adult starvation and disease-related malnutrition: a proposal for etiology-based diagnosis in the clinical practice setting from the International Consensus Guideline Committee. Clinical Nutrition 2010;29(2):151-3.

\section{Jones 2002}

Jones JM. The methodology of nutritional screening and assessment tools. Journal of Human Nutrition and Dietetics 2002;15:59-71.

\section{Katz 1963}

Katz S, Ford AB, Moskowitz RW, Jackson BA, Jaffe MW. Studies of illness in the aged: the index of ADL, a standardised measure of biological and psychological function. JAMA 1963;185:914-19.

\section{Kelly 2000}

Kelly IE, Tessier S, Cahill A, Morris SE, Crumley A, McLaughlin D, et al. Still hungry in hospital: identifying malnutrition in acute hospital admissions. Quarterly Journal of Medicine 2000;93(2):93-8.

\section{Khan 1981}

Khan MA, Hackler LR. Evaluation of food selection patterns and preferences. CRC Critical Reviews in Food Science and Nutrition 1981;15(2):129-53.

\section{Kirkham 2010}

Kirkham JJ, Dwan KM, Altman DG, Gamble C, Dodd S, Smyth R, et al. The impact of outcome reporting bias in randomised controlled trials on a cohort of systematic reviews. BMJ 2010;340:c365.

\section{Kubrak 2007}

Kubrak C, Jensen L. Critical evaluation of nutrition screening tools recommended for oncology patients. Cancer Nursing 2007;30(5):E1-6.

\section{Lambert 2010}

Lambert MA, Potter JM, McMurdo MET. Nutritional Supplementation for older people. Reviews in Clinical Gerontology 2010;20:317-26. 


\section{Lawton 1969}

Lawton MP, Brody EM. Assessment of older people: self maintaining and instrumental activities of daily living. The Gerontologist 1969;9(3):179-86.

\section{Lawton 1972}

Lawton MP (chapter). The Dimensions of Morale (chapter). Research planning and action for the elderly. Kent D, Kastenbaum R, Sherwood S. New York: Behavioural Publications, 1972.

\section{Lean 2008}

Lean M, Wiseman M. Malnutrition in hospitals. BMJ 2008;336(7639):290.

\section{Lennard-Jones 1992}

Lennard-Jones JE. A positive approach to nutrition as treatment: report of a working party chaired by Professor JE Lennard-Jones on the role of enteral and parenteral feeding in hospital and at home. London: King's Fund Centre, 1992.

\section{Liberati 2009}

Liberati A, Altman DG, Tetzlaff J, Mulrow C, Gøtzsche PC, loannidis JPA, et al. The PRISMA statement for reporting systematic and meta-analyses of studies that evaluate interventions: explanation and elaboration. Public Library of Science Medicine 1999;6(7):1-28.

\section{LPP 2009}

London Procurement Programme (LPP). Quality, Innovation, Productivity, Prevention (QIPP). Appropriate community oral nutritional supplementation, 2009. www.evidence.nhs.uk/qipp (accessed 16 March 2012).

\section{Lupton 1996}

Lupton D. Food, the Body and the Self. London: Sage, 1996.

\section{Mahoney 1965}

Mahoney FI, Bathel DW. Functional evaluation; the Barthel index. Maryland State Medical Journal 1965;14:61-5.

\section{Martyn 1998}

Martyn CN, Winter PD, Coles SJ, Edington J. Effect of nutritional status on use of health care resources by patients with chronic disease living in the community. Clinical Nutrition 1998;17(3):119-23.

\section{McCarron 2010}

McCarron J, Baldwin C, Weekes CE. Subjective assessment rather than systematic screening to identify malnourished patients for referral to a dietitian. Proceedings of the Nutrition Society 2010;69:E570.

\section{McKenzie 2014}

McKenzie J, Ryan R, Di Tanna GL. Cochrane Consumers and Communication Review Group: cluster randomised controlled trials. cccrg.cochrane.org March 2014.

\section{McMahon 2000}

McMahon K, Brown JK. Nutritional screening and assessment. Seminars in Oncology Nursing 2000;16:106-12.

\section{McQuestion 2011}

McQuestion M, Fitch M, Howell D. The changed meaning of food: physical, social and emotional loss for patients having received radiation treatment for head and neck cancer. European Journal of Oncology Nursing 2011;15(2):145-51.

\section{McWhirter 1994}

McWhirter JP, Pennington CR. Incidence and recognition of malnutrition in hospital. BMJ 1994;308(6934):945-8.

\section{Meader 2014}

Meader N, King K, Llewellyn A, Norman G, Brown J, Rodgers M, et al. A checklist designed to aid consistency and reproducibility of GRADE assessments: development and pilot validation. Systematic Reviews 2014;3:82.

\section{Milne 2009}

Milne AC, Potter J, Vivanti A, Avenell A. Protein and energy supplementation in elderly people at risk from malnutrition. Cochrane Database of Systematic Reviews 2009, Issue 2. [DOI: 10.1002/14651858.CD003288.pub3]

\section{Mueller 2011}

Mueller C, Compher C, Druyan ME, the American Society for Parenteral and Enteral Nutrition (ASPEN) Board of Directors. (Clinical Guidelines) Nutrition screening, assessment and intervention in adults. Journal of Parenteral and Enteral Nutrition 2011;35(1):16-24.

\section{Naber 1997}

Naber TH, Schermer T, de Bree A, Nusteling K, Eggink L, Kruimel JW, et al. Prevalence of malnutrition in nonsurgical hospitalized patients and its association with disease complications. American Journal of Clinical Nutrition 1997;66(5):1232-9.

\section{NCCAC 2006}

National Collaborating Centre for Acute Care (NCCAC). Nutrition Support in Adults: Oral Nutrition Support, Enteral Tube Feeding and Parental Nutrition. NICE Clinical Guideline 32. London, UK: National Collaborating Centre for Acute Care, 2006.

\section{Norman 2008}

Norman K, Pichard C, Lochs H, Pirlich M. Prognostic impact of disease-related malnutrition. Clinical Nutrition 2008;27(1):5-15

\section{RCON 2008}

Royal College of Nursing (RCON). Enhancing Nutritional Care. London: Royal College of Nursing, 2008.

\section{Reilly 1995}

Reilly HM, Martineau JK, Moran A, Kennedy H. Nutritional screening - evaluation and implementation of a simple Nutrition Risk Score. Clinical Nutrition 1995;14(5):269-73.

\section{RevMan 2014 [Computer program]}

The Nordic Cochrane Centre, The Cochrane Collaboration. Review Manager (RevMan). Version 5.3. Copenhagen: The Nordic Cochrane Centre, The Cochrane Collaboration, 2014. 


\section{Russell 2007}

Russell CA. The impact of malnutrition on healthcare costs and economic considerations for the use of oral nutritional supplements. Clinical Nutrition Supplements 2007;2(1):25-32.

\section{Schünemann 2011}

Schünemann HJ, Oxman AD, Higgins JPT, Vist GE, Glasziou P, Guyatt GH. Chapter 11: Presenting results and 'Summary of findings' tables. In: Higgins JPT, Green S (editors), Cochrane Handbook for Systematic Reviews of Interventions Version 5.1.0 (updated March 2011). The Cochrane Collaboration, 2011. Available from www.handbook.cochrane.org.

\section{Scrimshaw 2003}

Scrimshaw NS. Historical concepts of interactions, synergism and antagonism between nutrition and infection. Journal of Nutrition 2003;133(1):316S-21S.

\section{Silver 2009}

Silver HJ. Oral strategies to supplement older adults' dietary intakes: comparing the evidence. Nutrition Reviews 2009;67(1):21-31.

\section{Sonn 1996}

Sonn U. Longitudinal studies of dependence in daily life activities among elderly persons. Scandinavian Journal of Rehabilation Medicine Supplements 1996;34:1-35.

\section{Sterne 2011}

Sterne JA, Sutton AJ, loannidis JP, Terrin N, Jones DR, Lau J, et al. Recommendations for examining and interpreting funnel plot asymmetry in meta-analyses of randomised controlled trials. BMJ 2011;343:d4002.

\section{Stratton 2003}

Stratton RJ, Green CJ, Elia M. Disease related malnutrition: an evidence-based approach to treatment. Wallingford, UK: CABI Publishing, 2003.

\section{Stratton 2007}

Stratton RJ, Elia M. A review of reviews: a new look at the evidence for oral nutritional supplements in clinical practice. Clinical Nutrition Supplements 2007;2(1):5-23.

\section{CHARACTERISTICS OF STUDIES}

Characteristics of included studies [ordered by study ID]

\section{The Malnutrition Task Force 2013}

The Malnutrition Task Force. Prevention and Early Intervention of Malnutrition in Later Life. Best Practice Principles and Implementation Guide: A Local Community Approach. London: The Malnutrition Task Force, 2013.

\section{Van Campen 1998}

Van Campen C, Kerkstra A. Perceived quality of life of elderly somatic nursing-home patients. Construction of a measuring instrument. Tijdschrift voor Gerenterologie en Geriatrie 1998;29:11-18.

\section{Volkert 2006}

Volkert D, Berner YN, Berry E, Cederholm T, Coti Bertrand P, Milne $A$, et al. ESPEN guidelines on enteral nutrition: geriatrics. Clinical Nutrition 2006;25(2):330-60.

\section{Ware 1992}

Ware JE, Sherbourne CD. The MOS 36 item short form health survey (SF-36). Conceptual framework and item selection. Medical Care 1992;30(6):473-483.

\section{Weekes 1999}

Weekes CE. The incidence of malnutrition in medical patients admitted to a hospital in South London. Proceedings of Nutrition Society 1999;58:126A.

\section{Weekes 2009}

Weekes CE, Spiro A, Baldwin C, Whelan K, Thomas JE, Parkin D, et al. A review of the evidence for the impact of improving nutritional care on nutritional and clinical outcomes and cost. Journal of Human Nutrition and Dietetics 2009;22(4):324-35.

\section{References to other published versions of this review Gibbs 2012}

Gibbs M, Baldwin C, Weekes CE. Supportive interventions for enhancing dietary intake in malnourished or nutritionally atrisk adults. Cochrane Database of Systematic Reviews 2012, Issue 5. [DOI: 10.1002/14651858.CD009840]

* Indicates the major publication for the study

Barton 2000

Methods Cross-over randomised controlled clinical trial: this trial included 3 groups, 2 of which were randomised to treatment or control and one other where it was unclear whether there was randomisation

Randomisation ratio: not stated but appears to be $1: 1$

Superiority design

Participants

35 participants ( 27 randomised to intervention or control, 8 received cooked breakfast), 13 male, 22 female, mean age 75-78 depending on group; no details of nutritional status at baseline

Inclusion criteria: not stated 
Barton 2000 (Continued)

Exclusion criteria: not stated

Diagnostic criteria: elderly hospitalised patients in a rehabilitation ward, 19 of 35 had had a stroke

$\begin{array}{ll}\text { Interventions } & \text { Portion size decreased by } 20 \% \text { but fortified to achieve overall daily energy provision increased by } 200 \\ \text { kcal versus normal hospital menu. An additional group given normal hospital menu plus cooked break- } \\ \text { fast }\end{array}$

Number of trial centres: 1

Treatment before trial: not stated but assume normal hospital diet

\begin{tabular}{ll}
\hline Outcomes & Outcomes reported in abstract of publication: food wastage, energy and protein intake \\
\hline Study details & Location: Nottingham, UK \\
Year: unclear & Setting: $22-$ bedded rehab ward \\
Was trial terminated early: no
\end{tabular}

Publication details Language of publication: English

Funding: not stated

Publicaton status: peer review journal

\begin{tabular}{ll}
\hline Stated aim for study & $\begin{array}{l}\text { Quote from publication: "To compare food wastage and intake between the normal hospital menu and } \\
\text { one where more energy dense, but smaller portions were provided" }\end{array}$ \\
\hline Notes & - \\
\hline
\end{tabular}

Risk of bias

\begin{tabular}{|c|c|c|}
\hline Bias & Authors' judgement & Support for judgement \\
\hline $\begin{array}{l}\text { Random sequence genera- } \\
\text { tion (selection bias) }\end{array}$ & Unclear risk & $\begin{array}{l}\text { Quote from publication: "randomly allocated to receive either normal menu } \\
\text { or reduced portion fortified menu". } \\
\text { Comment: no details whether the third group was included in the randomisa- } \\
\text { tion \& insufficient detail provided of randomisation method }\end{array}$ \\
\hline $\begin{array}{l}\text { Allocation concealment } \\
\text { (selection bias) }\end{array}$ & Unclear risk & Comment: no information provided \\
\hline $\begin{array}{l}\text { Blinding of participants } \\
\text { and personnel (perfor- } \\
\text { mance bias) } \\
\text { All outcomes }\end{array}$ & Low risk & $\begin{array}{l}\text { Quote from publication: "patients and staff were blind as to which menu each } \\
\text { patient was following" } \\
\text { Comment: those receiving the cooked breakfast rather than cereal could not } \\
\text { have been blinded }\end{array}$ \\
\hline $\begin{array}{l}\text { Blinding of outcome as- } \\
\text { sessment (detection bias) } \\
\text { All outcomes }\end{array}$ & Unclear risk & Comment: no information provided \\
\hline
\end{tabular}

\begin{tabular}{|c|c|c|}
\hline $\begin{array}{l}\text { Incomplete outcome data } \\
\text { (attrition bias) }\end{array}$ & Unclear risk & $\begin{array}{l}\text { Comment: no detailed information provided. Data on } 19 \text { of } 27 \text { randomised } \\
\text { participants provided but no information on attrition }\end{array}$ \\
\hline
\end{tabular}

All outcomes participants provided but no information on attrition

Selective reporting (re- Low risk $\quad$ Comment: data presented on all three stated outcomes
porting bias)


Barton 2000 (Continued)
Other bias
Unclear risk
Comment: no information on baseline characteristics of populations apart from age and gender

Beck 2002

\begin{tabular}{|c|c|}
\hline Methods & $\begin{array}{l}\text { Parallel randomised controlled clinical trial } \\
\text { Randomisation ratio: } 1: 1: 1 \\
\text { Superiority design }\end{array}$ \\
\hline Participants & $\begin{array}{l}36 \text { care home residents; } 14 \text { male; } 22 \text { female; mean age } 81 \text { (range } 76-86 \text { ) years } \\
\text { Inclusion criteria: resident in a care home; aged }>65 \text { years } \\
\text { Exclusion criteria: in terminal condition } \\
\text { Diagnostic criteria: not specified }\end{array}$ \\
\hline Interventions & $\begin{array}{l}\text { Home-made oral supplement ( } 240 \mathrm{kcal} / \mathrm{serving}) \text { provided in the evening } \\
\text { Number of trial centres: } 1 \\
\text { Treatment before trial: none }\end{array}$ \\
\hline Outcomes & Outcomes reported in abstract of publication: energy intake and body weight \\
\hline Study details & $\begin{array}{l}\text { Run-in period: none } \\
\text { Was trial terminated early: no }\end{array}$ \\
\hline
\end{tabular}

Publication details

\section{Language of publication: English}

Funding: non-commercial funding - Health Insurance Foundation Grant

Publication status: peer review journal

$\begin{array}{ll}\text { Stated aim for study } & \text { Quote from publication: "To examine the effect of a home-made oral supplement on body weight and } \\ \text { energy intake of old people residing in a nursing home with MNA scores less than or equal to } 23.5 "\end{array}$

Notes -

\section{Risk of bias}

\begin{tabular}{lll}
\hline Bias & Authors' judgement & Support for judgement \\
\hline $\begin{array}{l}\text { Random sequence genera- } \\
\text { tion (selection bias) }\end{array}$ & Unclear risk & $\begin{array}{l}\text { Quote from paper: "Participants.... were randomly allocated (block random- } \\
\text { ization) to two groups" } \\
\text { Comment: insufficient detail of method provided }\end{array}$ \\
\hline $\begin{array}{l}\text { Allocation concealment } \\
\text { (selection bias) }\end{array}$ & Unclear risk & Comment: not described \\
\hline $\begin{array}{l}\text { Blinding of participants } \\
\text { and personnel (perfor- } \\
\text { mance bias) } \\
\text { All outcomes }\end{array}$ & Unclear risk & Comment: not described \\
\hline
\end{tabular}


Beck 2002 (Continued)

Blinding of outcome as- Unclear risk Comment: not described sessment (detection bias)

All outcomes

Incomplete outcome data Unclear risk Comment: not fully described
(attrition bias)

All outcomes

\begin{tabular}{lll}
\hline $\begin{array}{l}\text { Selective reporting (re- } \\
\text { porting bias) }\end{array}$ & Low risk & Comment: all outcomes reported \\
\hline Other bias & Unclear risk & Comment: not fully described \\
\hline
\end{tabular}

Bouillanne 2013

\begin{tabular}{ll}
\hline Methods & Parallel randomised controlled clinical trial \\
& Randomisation ratio: $1: 1$ \\
& Superiority design \\
\hline Participants & 73 hospitalised elderly; 46 female; 27 male; mean age intervention $84.1(95 \% \mathrm{Cl} 82$ to 86$) ;$ control 85.7 \\
$(95 \% \mathrm{Cl} 84$ to 88$)$ years \\
Inclusion criteria: albumin $25-35 \mathrm{~g} / \mathrm{L} ; \mathrm{BMI}<22 \mathrm{~kg} / \mathrm{m}^{2}$ and $/$ or weight loss $>10 \%$ in $6 \mathrm{months}$ and/or \\
MNA $\leq 23.5$ \\
Exclusion criteria: not specified \\
Diagnostic criteria: admitted to geriatric intermediate care unit
\end{tabular}

Interventions

Number of trial centres: 1

Treatment before trial: none

Pulse diet i.e. $78 \%$ daily protein requirements provided at noon meal

\begin{tabular}{ll}
\hline Outcomes & Outcomes reported in abstract of publication: body composition, handgrip strength and ADL score \\
\hline Study details & Run-in period: none \\
& Was trial terminated early: no \\
\hline Publication details & Language of publication: English \\
& $\begin{array}{l}\text { Funding: non-commercial funding - French Ministry of Health } \\
\text { Publication status: peer review journal }\end{array}$ \\
\hline Stated aim for study & $\begin{array}{l}\text { Quote from publication: "To evaluate the efficacy of a new nutritional strategy, termed protein pulse } \\
\text { feeding" }\end{array}$ \\
\hline Notes & - \\
\hline Risk of bias & Authors' judgement Support for judgement \\
\hline Bias
\end{tabular}


Bouillanne 2013 (Continued)

Random sequence genera- Unclear risk Quote from paper: "A randomization procedure was used (EXCEL 2003....." tion (selection bias)

Comment: insufficient detail of the method provided

\begin{tabular}{lll}
\hline $\begin{array}{l}\text { Allocation concealment } \\
\text { (selection bias) }\end{array}$ & Unclear risk & Comment: not described \\
\hline $\begin{array}{l}\text { Blinding of participants } \\
\text { and personnel (perfor- } \\
\text { mance bias) }\end{array}$ & Unclear risk & Comment: not described \\
All outcomes & & \\
\hline
\end{tabular}

Blinding of outcome as- Unclear risk Comment: not described sessment (detection bias)

All outcomes

Incomplete outcome data Low risk Comment: all participants fully accounted for
(attrition bias)

(attrition bias)

All outcomes

Selective reporting (re- Low risk Comment: all outcomes fully reported
porting bias)

Other bias Low risk

Comment: baseline characteristics fully compared; serum albumin higher and body cell mass index and skeletal muscle mass index are lower in the pulse diet group

Bourdel-Marchasson 2000

\begin{tabular}{ll}
\hline Methods & Cluster-randomised controlled clinical trial \\
& Randomisation ratio: $1: 1$ \\
& Superiority design \\
\hline Participants & 672 critically ill elderly participants; $\mathrm{N}=295$ intervention $(199 \mathrm{female}, 96 \mathrm{male}) ; \mathrm{N}=377 \mathrm{control}(238 \mathrm{fe}-$ \\
& male, 139 male). Mean age in intervention group $=83.6$ yrs (SD 7.3) and mean age in the intervention \\
& group $=83.0$ yrs (SD 7.1) \\
& $\begin{array}{l}\text { Inclusion criteria: wards inclusion: }>40 \% \text { of participants over age } 65 \text { yrs and nurses able to guarantee } \\
\text { significant involvement in the trial. Older than } 65 \text { yrs, in acute phase of a critical illness, unable to move }\end{array}$
\end{tabular}
by themselves, unable to eat independently on admission

Exclusion criteria: pressure ulcers at admission

Diagnostic criteria: critically ill inpatients

Interventions

Intervention group received standard diet of $1800 \mathrm{kcal} /$ day plus 2 oral nutritional supplements of 200 kcal each, one with breakfast and the other mid afternoon. Control group received standard diet of $1800 \mathrm{kcal} /$ day

Number of trial centres: unclear

Treatment before trial: none 
Bourdel-Marchasson 2000 (Continued)
Study details
Run-in period: none
Was trial terminated early: no

\section{Publication details Language of publication: English}

Funding: non-commercial/other funding - Projet Hospitalier de Recherche Clinique, Ministere de la Sante et de l'Action Humanitaire, Direction Generale de la Sante and the Direction des Hopitaux

Publication status: peer review journal

Stated aim for study

Quote from publication: "The purpose of the present study was to assess the effects of nutritional supplementation ( $400 \mathrm{kcal} / \mathrm{day}$ ) for 15 days on dietary intake and on pressure ulcer development in critically ill older patients; 672 subjects older than 65 years were included"

\section{Notes \\ Risk of bias}

\begin{tabular}{lll}
\hline Bias & Authors' judgement & Support for judgement \\
\hline $\begin{array}{l}\text { Random sequence genera- } \\
\text { tion (selection bias) }\end{array}$ & Unclear risk & $\begin{array}{l}\text { Quote from paper: "Nineteen wards were then selected and stratified accord- } \\
\text { ing their speciality.... These wards were then randomised in two groups accord- } \\
\text { ing to the nutritional intervention } \\
\text { Comment: insufficient detail of method provided }\end{array}$ \\
\hline $\begin{array}{l}\text { Allocation concealment } \\
\text { (selection bias) }\end{array}$ & Unclear risk & Comment: not described \\
\hline $\begin{array}{l}\text { Blinding of participants } \\
\text { and personnel (perfor- } \\
\text { mance bias) } \\
\text { All outcomes }\end{array}$ & Unclear risk & Comment: not described \\
\hline
\end{tabular}

\begin{tabular}{|c|c|c|}
\hline $\begin{array}{l}\text { Blinding of outcome as- } \\
\text { sessment (detection bias) }\end{array}$ & Unclear risk & Comment: not described \\
\hline
\end{tabular}

\begin{tabular}{|c|c|c|}
\hline $\begin{array}{l}\text { Incomplete outcome data } \\
\text { (attrition bias) }\end{array}$ & Unclear risk & $\begin{array}{l}\text { Comment: } 25 \text { deaths in intervention group and } 22 \text { in the usual care. Other at- } \\
\text { trition not described }\end{array}$ \\
\hline
\end{tabular}

All outcomes

Low risk

Comment: all outcomes reported

Selective reporting (re- Low risk Comment: all outcomes reported
porting bias)

Other bias High risk

\section{Assessment of risk of bias in cluster-randomised trials}

(1) Recruitment bias: unclear

(2) Baseline imbalance: yes (serum albumin at baseline, weight, Norton score, Kuntzmann mean score)

(3) Loss of clusters: unclear

(4) Incorrect analysis: no

(5) Comparability with individually randomised trials/different types of clus-

ters: different types of clusters 
Brouillette 1991

\begin{tabular}{ll} 
Methods & Parallel randomised controlled clinical trial \\
& Randomisation ratio: $1: 1$ \\
& Superiority design \\
\hline Participants & 16 participants; 14 female; 2 male; mean age Intervention 80 (SD 6.4); control 87 (SD 6.8) years \\
Inclusion criteria: care home residents \\
Exclusion criteria: cancer; severe Gl disorder and/or oral disorder; extreme dietary restriction or other \\
conditions that affect ability to eat or feed themselves
\end{tabular}

Diagnostic criteria: not specified

\begin{tabular}{ll}
\hline Interventions & Exposure to olfactory stimuli prior to meals + other activities \\
& Number of trial centres: 1 \\
& Treatment before trial: none \\
\hline Outcomes & Outcomes reported in abstract of publication: olfactory acuity and attention level \\
\hline Study details & Run-in period: none \\
& Was trial terminated early: no \\
\hline
\end{tabular}

Publication details Language of publication: English

Funding: not stated

Publication status: peer review journal

\begin{tabular}{ll}
\hline Stated aim for study & $\begin{array}{l}\text { Quote from publication: "To test whether odours can influence the desire to eat and therefore increase } \\
\text { caloric intake" }\end{array}$ \\
\hline Notes & - \\
\hline
\end{tabular}

Risk of bias

\begin{tabular}{lll}
\hline Bias & Authors' judgement & Support for judgement \\
\hline $\begin{array}{l}\text { Random sequence genera- } \\
\text { tion (selection bias) }\end{array}$ & Unclear risk & $\begin{array}{l}\text { Quote from publication: "From the remaining pool, 20 subjects were selected } \\
\text { for the research ... The 20 subjects were assigned randomly to either the exper- } \\
\text { imental or control group" }\end{array}$
\end{tabular}

Comment: no details on randomisation procedure

\begin{tabular}{lll}
\hline $\begin{array}{l}\text { Allocation concealment } \\
\text { (selection bias) }\end{array}$ & Unclear risk & Comment: not described \\
\hline $\begin{array}{l}\text { Blinding of participants } \\
\text { and personnel (perfor- } \\
\text { mance bias) }\end{array}$ & Low risk & $\begin{array}{l}\text { Comment: during the research period it is stated that "the research assistant } \\
\text { was unaware of group assignment" }\end{array}$ \\
All outcomes & &
\end{tabular}

Blinding of outcome as- Low risk sessment (detection bias)
Comment: during the research period it is stated that "the research assistant was unaware of group assignment" 
Brouillette 1991 (Continued)

All outcomes

Incomplete outcome data Low risk Comment: all dropouts fully accounted for
(attrition bias)

All outcomes

\begin{tabular}{lll}
\hline $\begin{array}{l}\text { Selective reporting (re- } \\
\text { porting bias) }\end{array}$ & Low risk & Comment: all outcomes fully reported \\
\hline Other bias & Low risk & Comment: baseline characteristics reported and groups comparable \\
\hline
\end{tabular}

\section{Castellanos 2009}

\section{Methods}

Cross-over randomised controlled clinical trial: each individual was tested under three menu conditions ( 2 different interventions and 1 control)

Randomisation ratio: not stated

\section{Superiority design}

\section{Participants}

39 participants ( 4 died and 2 withdrew before inclusion, complete data on 26 following attrition). 10 male, 23 female, mean age 87.3 (SD 8.6) years, mean BMI 25.1 (SD 3.6)

Inclusion criteria: nursing home residents

Exclusion criteria: < 60 years, hospice patients, on tube feeding, renal diet, pureed diet, thickened liquids, ate only in their room, required feeding assistance.

Diagnostic criteria: nursing home residents

$\begin{array}{ll}\text { Interventions } & 2 \text { breakfast and } 2 \text { lunch foods fortified to improve energy and protein content (hot cereal and juice } \\ \text { breakfast, soup and side dish at lunch) versus } 2 \text { lunch foods only fortified versus normal menu }\end{array}$

\section{Number of trial centres: 1}

Treatment before trial: not stated, assume usual menu

Outcomes Outcomes reported in abstract of publication: energy and protein intake

\begin{tabular}{ll}
\hline Study details & Location: Florida, USA \\
Year: mid 2000's \\
Setting: nursing home \\
Run-in period: not stated \\
Was trial terminated early: no \\
\hline Publication details & Language of publication: English \\
& Other funding: Juice drinks donated by Lyons Magnus, Fresno CA \\
& Publication status: peer review journal \\
\hline
\end{tabular}


Castellanos 2009 (Continued)

Stated aim for study Quote from publication: "the study objective was to determine whether energy and protein enhancement of a small number of menu items would result in increased 3-meal (breakfast, lunch and supper) calorie and protein intakes in long term care residents"

\title{
Notes
}

\section{Risk of bias}

Bias Authors' judgement Support for judgement

Random sequence genera- Unclear risk tion (selection bias)
Quote from paper: "Using a single blind randomised cross over design, each subject was tested under three menu conditions"

Comment: insufficient details of method provided

\begin{tabular}{lll}
\hline $\begin{array}{l}\text { Allocation concealment } \\
\text { (selection bias) }\end{array}$ & Unclear risk & Comment: not stated \\
\hline $\begin{array}{l}\text { Blinding of participants } \\
\text { and personnel (perfor- } \\
\text { mance bias) }\end{array}$ & Unclear risk & $\begin{array}{l}\text { Comment: described as single blind, unclear whether residents or staff were } \\
\text { blinded }\end{array}$ \\
All outcomes & \\
\hline
\end{tabular}

\begin{tabular}{lll}
\hline $\begin{array}{l}\text { Blinding of outcome as- } \\
\text { sessment (detection bias) } \\
\text { All outcomes }\end{array}$ & Unclear risk & Comment: not stated \\
\hline $\begin{array}{l}\text { Incomplete outcome data } \\
\text { (attrition bias) } \\
\text { All outcomes }\end{array}$ & Low risk & Comment: complete data included in figure 1 \\
\hline $\begin{array}{l}\text { Selective reporting (re- } \\
\text { porting bias) }\end{array}$ & High risk & $\begin{array}{l}\text { Comment: results for the whole group are not reported according to initial } \\
\text { randomisation. Only data for post hoc separation of the whole group into large } \\
(\geq 1150 \text { kcal in 3 meals) and small eaters (<1150 kcal in 3 meals) were reported. } \\
\text { This excludes 7 participants with incomplete data }\end{array}$ \\
\hline
\end{tabular}

\begin{tabular}{|c|c|c|}
\hline Other bias & Unclear risk & $\begin{array}{l}\text { Comment: baseline characteristics reported in table } 1 \text { for large and smaller } \\
\text { eaters but not for the whole group }\end{array}$ \\
\hline
\end{tabular}

\section{Chang 2005}

\section{Methods Cluster-randomised controlled clinical trial}

Randomisation ratio: $1: 1$

\section{Superiority design}

\section{Participants}

\begin{abstract}
67 nursing assistants randomised, 36 nursing assistants took part in the observation of mealtimes part of the study; $\mathrm{N}=20$ intervention (all female); $\mathrm{N}=16$ control (14 female and 2 male)

and 36 care home residents with dementia (mean age 84.2 (SD 4) intervention and 72 (SD 5.8) years in control)
\end{abstract}

Inclusion criteria: nursing assistants had to have worked at least 6 months in the same long-term care facility and able to communicate in either Mandarin, Taiwanese or English. Residents diagnosed with dementia, having an eating problem and needing assistance

Exclusion criteria: not stated 
Chang 2005 (Continued)

Diagnostic criteria: dementia

\begin{tabular}{ll}
\hline Interventions & Feeding skills training programme for nursing assistants versus usual care \\
& Number of trial centres: 1 \\
& Treatment before trial: not stated \\
\hline Outcomes & $\begin{array}{l}\text { Outcomes reported in abstract of publication: knowledge, attitude and behaviour of nursing assis- } \\
\text { tants, Edinburgh Feeding Evaluation in Dementia Score; food intake and eating time of participants }\end{array}$ \\
\hline Study details & Run-in period: not stated \\
Was trial terminated early: no
\end{tabular}

Publication details Language of publication: English

Funding: commercial and non-commercial - Sigma Theta Tau International-Alpha Mu Chapter and the Alumni Association of the FPB School of Nursing and National health Research Institute

Publication status: peer review journal

Stated aim for study

Quote from publication: "to provide a feeding skills training programme for nursing assistants in a Taiwanese dementia-specialised long term care facility and to test the effects of this feeding skills training programme on the outcomes of nursing assistants and dementia patients".

\begin{tabular}{|c|c|c|}
\hline Notes & - & \\
\hline \multicolumn{3}{|l|}{ Risk of bias } \\
\hline Bias & Authors' judgement & Support for judgement \\
\hline \multirow[t]{2}{*}{$\begin{array}{l}\text { Random sequence genera- } \\
\text { tion (selection bias) }\end{array}$} & Low risk & $\begin{array}{l}\text { Quote from paper: "Two convenience-chosen, dementia-specialised, long- } \\
\text { term care facilities in North Taiwan were randomly assigned into either a con- } \\
\text { trol or treatment group by flipping a coin" }\end{array}$ \\
\hline & & $\begin{array}{l}\text { Comment: implies that the study may be cluster randomised but not clear } \\
\text { from the information provided }\end{array}$ \\
\hline $\begin{array}{l}\text { Allocation concealment } \\
\text { (selection bias) }\end{array}$ & Unclear risk & Comment: insufficient information to make a judgement \\
\hline $\begin{array}{l}\text { Blinding of participants } \\
\text { and personnel (perfor- } \\
\text { mance bias) } \\
\text { All outcomes }\end{array}$ & Unclear risk & Comment: insufficient detail \\
\hline
\end{tabular}

\begin{tabular}{ll}
\hline Blinding of outcome as- & Unclear risk \\
sessment (detection bias) & \\
All outcomes & \\
\hline
\end{tabular}

Incomplete outcome data High risk Comment: data not presented on 16/36 individuals with no reasons why
(attrition bias)

All outcomes

\begin{tabular}{lll}
\hline $\begin{array}{l}\text { Selective reporting (re- } \\
\text { porting bias) }\end{array}$ & Low risk & Comment: all outcomes reported \\
\hline Other bias & Unclear risk & $\begin{array}{l}\text { Comment: insufficient data on baseline characteristics of nursing assistants } \\
\text { and of participants; nursing assistants in usual care had significantly longer }\end{array}$
\end{tabular}


Randomisation ratio: $1: 1$

\section{Superiority design}

Participants
female; mean age 71 (SD 13))
Inclusion criteria: people admitted with recent stroke, (first or recurrent stroke no more than 7 days
before admission), if they passed swallow screen, the responsible clinician was uncertain whether to
use oral nutritional supplements and the participant or relative consented to enrolment

Exclusion criteria: subarachnoid haemorrhage

Diagnostic criteria: stroke patients

\begin{tabular}{|c|c|}
\hline Interventions & $\begin{array}{l}\text { Intervention group received normal hospital diet plus oral protein energy supplements ( } 360 \mathrm{~mL}) \text { pre- } \\
\text { scribed on drug administration charts; usual care received normal hospital diet until discharge } \\
\text { Number of trial centres: } 125 \text { hospitals in } 15 \text { different countries } \\
\text { Treatment before trial: none }\end{array}$ \\
\hline Outcomes & $\begin{array}{l}\text { Outcomes reported in abstract of publication: death, poor outcome (modified Rankin scale grade } \\
3-5)\end{array}$ \\
\hline Study details & $\begin{array}{l}\text { Run-in period: not stated } \\
\text { Was trial terminated early: no }\end{array}$ \\
\hline Publication details & $\begin{array}{l}\text { Language of publication: English } \\
\text { Funding: commercial/other funding- Health Technology Assessment Board of NHS Research and De- } \\
\text { velopment in the UK, the Stroke Association, the Chief Scientist Office of the Scottish Executive, and } \\
\text { Chest, Heart and Stroke, Scotland } \\
\text { Publication status: peer review journal }\end{array}$ \\
\hline Stated aim for study & $\begin{array}{l}\text { Quote from publication: "to establish whether routine oral nutritional supplements improve outcome } \\
\text { after stroke" }\end{array}$ \\
\hline Notes & $\begin{array}{l}\text { The FOOD (feed or ordinary diet) trials consisted of three RCTs, sharing the same randomisation, da- } \\
\text { ta collection, and follow-up systems, allowed co-enrolment, and aimed to compare the outcomes of } \\
\text { stroke patients in hospital. Dysphagic patients were enrolled into one or both of two trials: (1) early en- } \\
\text { teral tube feeding versus avoidance of tube feeding for at least } 7 \text { days; and (2) tube feeding via percu- } \\
\text { taneous endoscopic gastrostomy versus nasogastric tube. For this systematic review we describe the } \\
\text { outcomes of participants who were able to swallow }\end{array}$ \\
\hline
\end{tabular}

\section{Risk of bias}

Bias Authors' judgement Support for judgement


Dennis 2005 (Continued) $\begin{aligned} & \text { Random sequence genera- } \\ & \text { tion (selection bias) }\end{aligned} \quad \begin{aligned} & \text { Quote from publication: "the computer allocated the feeding regimen". Also, } \\ & \text { "A computer generated minimisation algorithm" was used }\end{aligned}$

\begin{tabular}{ll}
\hline $\begin{array}{l}\text { Allocation concealment } \\
\text { (selection bias) }\end{array}$ & Quote from publication: "the computer allocated the feeding regimen" \\
& $\begin{array}{l}\text { Comment: central allocation method ensured treatment allocation was con- } \\
\text { cealed until intervention was given }\end{array}$
\end{tabular}

\begin{tabular}{|c|c|c|}
\hline $\begin{array}{l}\text { Blinding of participants } \\
\text { and personnel (perfor- } \\
\text { mance bias) }\end{array}$ & Unclear risk & $\begin{array}{l}\text { Quote from publication: "Neither the randomising clinician, the clinical team, } \\
\text { nor patients were unaware of treatment allocation; doing so would have been } \\
\text { very difficult" }\end{array}$ \\
\hline
\end{tabular}

All outcomes

$\begin{array}{lll}\begin{array}{l}\text { Blinding of outcome as- } \\ \text { sessment (detection bias) }\end{array} & \text { High risk } & \begin{array}{l}\text { Quote from publication: "Follow-up was masked to treatment allocation (ex- } \\ \text { cept when patients or carers inadvertently divulged it to an interviewer, which } \\ \text { All outcomes }\end{array} \\ \text { was unusual but not systematically recorded)" }\end{array}$

\begin{tabular}{lll}
\hline $\begin{array}{l}\text { Incomplete outcome data } \\
\text { (attrition bias) } \\
\text { All outcomes }\end{array}$ & Low risk & $\begin{array}{l}\text { Comment: study attrition presented in a figure and all randomised partici- } \\
\text { pants accounted for }\end{array}$ \\
\hline $\begin{array}{l}\text { Selective reporting (re- } \\
\text { porting bias) }\end{array}$ & Low risk & Comment: all of the outcomes specified were reported \\
\hline Other bias & Unclear risk & $\begin{array}{l}\text { Comment: insufficient information to permit judgement of 'Low risk' or 'High } \\
\text { risk' }\end{array}$ \\
\hline
\end{tabular}

Duncan 2006

\begin{tabular}{|c|c|}
\hline Methods & $\begin{array}{l}\text { Parallel randomised controlled clinical trial } \\
\text { Randomisation ratio: not stated but assume 1:1 } \\
\text { Superiority design }\end{array}$ \\
\hline Participants & $\begin{array}{l}318 \text { participants; } 100 \% \text { female; mean age intervention } 83.5 \text { and control } 83.6 \text { years } \\
\text { Inclusion criteria: women > } 65 \text { years admitted with acute hip fracture } \\
\text { Exclusion criteria: not stated } \\
\text { Diagnostic criteria: acute non-pathological hip fracture }\end{array}$ \\
\hline Interventions & $\begin{array}{l}\text { Intervention: additional personal attention of a dietetic assistant } \\
\text { Control: usual care } \\
\text { Number of trial centres: } 1 \\
\text { Treatment before trial: none }\end{array}$ \\
\hline Outcomes & $\begin{array}{l}\text { Outcomes reported in abstract of publication: } \\
\text { Primary: post-operative mortality in the acute trauma unit } \\
\text { Secondary: post-operative mortality at } 4 \text { months; length of hospital stay, energy intake and nutritional } \\
\text { status }\end{array}$ \\
\hline Study details & Location: Wales, UK \\
\hline
\end{tabular}


Duncan 2006 (Continued)

Year: May-August 2003

Setting: acute trauma ward

Run-in period: none

Was trial terminated early: no

\begin{tabular}{ll}
\hline Publication details & Language of publication: English \\
& $\begin{array}{l}\text { Funding: non-commercial funding - Womens Royal Volunteer Service + British Dietetic Association, } \\
\text { novations in Care Shire Pharmaceuticals, Wales Office of Research \& Development }\end{array}$ \\
& Publication status: peer review journal \\
\hline Stated aim for study & $\begin{array}{l}\text { Quote from publication: "To examine how improved attention to nutrition status and dietary intake } \\
\text { achieved through the employment of dietetic assistants will affect post-operative clinical outcome } \\
\text { among elderly women with hip fracture" }\end{array}$
\end{tabular}

Notes

\section{Risk of bias}

\begin{tabular}{|c|c|c|}
\hline Bias & Authors' judgement & Support for judgement \\
\hline $\begin{array}{l}\text { Random sequence genera- } \\
\text { tion (selection bias) }\end{array}$ & Unclear risk & Comment: sequence generation not described \\
\hline $\begin{array}{l}\text { Allocation concealment } \\
\text { (selection bias) }\end{array}$ & Low risk & $\begin{array}{l}\text { Quote from paper: "sequentially numbered opaque sealed envelope method } \\
\text { in blocks of } 10 \text {, prepared by a member of staff not involved in the trial" }\end{array}$ \\
\hline $\begin{array}{l}\text { Blinding of participants } \\
\text { and personnel (perfor- } \\
\text { mance bias) } \\
\text { All outcomes }\end{array}$ & Unclear risk & Comment: not described \\
\hline $\begin{array}{l}\text { Blinding of outcome as- } \\
\text { sessment (detection bias) } \\
\text { All outcomes }\end{array}$ & Low risk & $\begin{array}{l}\text { Comment: assessments made by member of trial team blind to treatment al- } \\
\text { location and independent of dietitian and dietetic assistants }\end{array}$ \\
\hline $\begin{array}{l}\text { Incomplete outcome data } \\
\text { (attrition bias) } \\
\text { All outcomes }\end{array}$ & Low risk & Comment: fully described \\
\hline $\begin{array}{l}\text { Selective reporting (re- } \\
\text { porting bias) }\end{array}$ & Low risk & Comment: all specified outcomes reported \\
\hline Other bias & Low risk & Comment: baseline characteristics show groups are comparable \\
\hline
\end{tabular}

Essed 2007

\begin{tabular}{ll}
\hline Methods & Factorial randomised controlled clinical trial \\
& Randomisation ratio: $1: 1: 1: 1$ \\
& Superiority design \\
\hline Participants & 97 participants (83 completed); mean age 84.9-85.6 years (SD 5.7-8.5); 58 female and 25 male \\
\hline
\end{tabular}


Inclusion criteria: aged 65 years or older; resident of nursing home for more than 3 months; no terminal disease; no allergy to MSG; consuming meals provided by the nursing home at least 5 days/week

\section{Exclusion criteria:}

Diagnostic criteria: not stated

\begin{tabular}{ll}
\hline Interventions & Four arms; food sprinkled with 1. MSG 2. Flavour 3. MSG + Flavour 4. Maltodextrin (placebo) \\
& Number of trial centres: 3 care homes in the Netherlands \\
& Treatment before trial: not stated \\
\hline Outcomes & Outcomes reported in abstract of publication: energy intake and body weight \\
\hline Study details & Run-in period: two weeks \\
& Was trial terminated early: no \\
\hline Publication details & $\begin{array}{l}\text { Language of publication: English } \\
\text { Funding: non-commercial funding } \\
\text { Publication status: peer review journal }\end{array}$ \\
\hline Stated aim for study & $\begin{array}{l}\text { Quote from publication: "To determine whether daily addition of flavour and/or MSG to the animal pro- } \\
\text { tein part of a cooked meal for } 16 \text { weeks leads to an increase in energy intake of the cooked meal and an } \\
\text { increase in body weight" }\end{array}$ \\
\hline Notes & \begin{tabular}{l} 
- \\
\hline
\end{tabular}
\end{tabular}

\section{Risk of bias}

\begin{tabular}{lll}
\hline Bias & Authors' judgement & Support for judgement \\
\hline $\begin{array}{l}\text { Random sequence genera- } \\
\text { tion (selection bias) }\end{array}$ & Unclear risk & Quote from paper: participants were reported as being "randomly assigned" \\
& & Comment: insufficient detail of the method provided \\
\hline
\end{tabular}

$\begin{array}{ll}\begin{array}{l}\text { Allocation concealment } \\ \text { (selection bias) }\end{array} & \text { Unclear risk } \\ \text { Qugned". }\end{array}$

Comment: insufficient detail of the method provided

\begin{tabular}{|c|c|c|}
\hline $\begin{array}{l}\text { Blinding of participants } \\
\text { and personnel (perfor- } \\
\text { mance bias) } \\
\text { All outcomes }\end{array}$ & High risk & $\begin{array}{l}\text { Comment: single-blind i.e. participants were blinded but not research person- } \\
\text { nel }\end{array}$ \\
\hline
\end{tabular}

\begin{tabular}{lll}
\hline $\begin{array}{l}\text { Blinding of outcome as- } \\
\text { sessment (detection bias) } \\
\text { All outcomes }\end{array}$ & Unclear risk & Comment: not stated \\
\hline $\begin{array}{l}\text { Incomplete outcome data } \\
\text { (attrition bias) }\end{array}$ & Low risk & Comment: all participants accounted for \\
$\begin{array}{l}\text { All outcomes } \\
\text { Selective reporting (re- }\end{array}$ & Low risk & Comment: all outcomes specified in the methods are reported in the results \\
\hline \begin{tabular}{l} 
porting bias) \\
\hline
\end{tabular}
\end{tabular}


Essed 2007 (Continued)

Other bias Low risk Comment: baseline characteristics comparable and reported in Table 1

Essed 2009

$\begin{array}{ll}\text { Methods } & \text { Cross-over randomised controlled clinical trial } \\ \text { Randomisation ratio: } 1: 1 \\ \text { Superiority design }\end{array}$

Participants 53 nursing home residents (13 male: 40 female); aged 85.8 (SD 5.2) years

Inclusion criteria: > 65 years old; able to participate; good eyesight

Exclusion criteria: allergy to MSG; on sodium restricted diet; on anti-depressants; terminal illness

Diagnostic criteria: not stated

\begin{tabular}{|c|c|c|}
\hline \multirow[t]{4}{*}{ Interventions } & \multicolumn{2}{|c|}{ Intervention: hot meal including three foods with added salt and MSG } \\
\hline & \multicolumn{2}{|c|}{ Control: usual hot meals } \\
\hline & \multicolumn{2}{|c|}{ Number of trial centres: 1} \\
\hline & \multicolumn{2}{|c|}{ Treatment before trial: usual diet } \\
\hline Outcomes & \multicolumn{2}{|c|}{ Outcomes reported in abstract of publication: dietary intake } \\
\hline \multirow[t]{2}{*}{ Study details } & \multicolumn{2}{|l|}{ Run-in period: none } \\
\hline & \multicolumn{2}{|c|}{ Was study terminated early: not stated } \\
\hline \multirow[t]{3}{*}{ Publication details } & \multirow{3}{*}{\multicolumn{2}{|c|}{$\begin{array}{l}\text { Language of publication: English } \\
\text { Funding: non-commercial funding } \\
\text { Publication status: peer review journal }\end{array}$}} \\
\hline & & \\
\hline & & \\
\hline Stated aim for study & \multicolumn{2}{|c|}{$\begin{array}{l}\text { Quote from publication: "To determine whether or not an optimal preferred MSG concentration in sev- } \\
\text { eral foods increases intake in elderly people" }\end{array}$} \\
\hline Notes & \multicolumn{2}{|l|}{-} \\
\hline \multicolumn{3}{|l|}{ Risk of bias } \\
\hline Bias & Authors' judgement & Support for judgement \\
\hline \multirow{2}{*}{$\begin{array}{l}\text { Random sequence genera- } \\
\text { tion (selection bias) }\end{array}$} & \multirow[t]{2}{*}{ Unclear risk } & Quote from paper: described as: ".. in a random order" \\
\hline & & Comment: insufficient detail of the method provided \\
\hline \multirow{2}{*}{$\begin{array}{l}\text { Allocation concealment } \\
\text { (selection bias) }\end{array}$} & \multirow[t]{2}{*}{ Unclear risk } & Quote from paper: "The studies were carried out single blind" \\
\hline & & Comment: insufficient detail of the method provided \\
\hline $\begin{array}{l}\text { Blinding of participants } \\
\text { and personnel (perfor- } \\
\text { mance bias) }\end{array}$ & Unclear risk & Comment: not stated who was blinded \\
\hline
\end{tabular}


Essed 2009 (Continued)

All outcomes

Blinding of outcome as-
sessment (detection bias) $\quad$ Unclear risk Comment: not stated

sessment (detection bias)

All outcomes

Incomplete outcome data Low risk Comment: fully described
(attrition bias)

All outcomes

\begin{tabular}{lll}
\hline $\begin{array}{l}\text { Selective reporting (re- } \\
\text { porting bias) }\end{array}$ & Unclear risk & Comment: insufficient information to judge \\
\hline Other bias & Unclear risk & Comment: baseline characteristics reported \\
\hline
\end{tabular}

Faxen-Irving 2011

\begin{tabular}{|c|c|}
\hline Methods & $\begin{array}{l}\text { Parallel randomised controlled clinical trial } \\
\text { Randomisation ratio: } 1: 1 \\
\text { Superiority design }\end{array}$ \\
\hline Participants & $\begin{array}{l}71 \text { recently admitted geriatric patients; } \mathrm{N}=34 \text { intervention; } \mathrm{N}=37 \text { control. Mean age of all participants } \\
=84 \text { (SD 7.1) } \\
\text { Inclusion criteria: likelihood of hospital stay more than one week, }>65 \text { years old and able to give in- } \\
\text { formed consent } \\
\text { Exclusion criteria: pancreatitis, fat malabsorption, } \mathrm{BMI}>30 \mathrm{~kg} / \mathrm{m}^{2} \text {, and non-consent for participation } \\
\text { Diagnostic criteria: not stated }\end{array}$ \\
\hline Interventions & $\begin{array}{l}\text { Intervention group received a daily dose of } 3 \times 30 \mathrm{~mL} \text { fat emulsion at the same time as pharmaceutical } \\
\text { prescriptions. The usual care received usual care } \\
\text { Number of trial centres: } 1 \\
\text { Treatment before trial: not stated }\end{array}$ \\
\hline Outcomes & $\begin{array}{l}\text { Outcomes reported in abstract of publication: food intake, self-rated appetite, NRS, serum lipids, fat- } \\
\text { ty acid profiles }\end{array}$ \\
\hline Study details & $\begin{array}{l}\text { Run-in period: no } \\
\text { Was trial terminated early: no }\end{array}$ \\
\hline Publication details & $\begin{array}{l}\text { Language of publication: English } \\
\text { Funding: non-commercial/commercial funding- SHS International \& Nutricia (Sweden) and the Re- } \\
\text { gional Agreement on Medical Training \& Clinical Research between Stockholm County Council and the } \\
\text { Karolinska Institutet } \\
\text { Publicaton status: peer review journal }\end{array}$ \\
\hline Stated aim for study & $\begin{array}{l}\text { Quote from publication: "the effects on an oleic acid rich formula on energy intake and appetite were } \\
\text { studied" }\end{array}$ \\
\hline
\end{tabular}


Faxen-Irving 2011 (Continued)

Notes

\section{Risk of bias}

Bias Authors' judgement Support for judgement

Random sequence genera- Unclear risk tion (selection bias)

Quote from paper: "an open randomised controlled trial. Permutted blocks of 10 were employed for the randomisation. No stratification was used".

Comment: insufficient detail of method provided

\begin{tabular}{|c|c|c|}
\hline $\begin{array}{l}\text { Allocation concealment } \\
\text { (selection bias) }\end{array}$ & High risk & $\begin{array}{l}\text { Quote from publication: "Sealed envelopes, opened by the study nurses after } \\
\text { acceptance from the patients was received, were used to allocate individuals } \\
\text { to intervention or control" }\end{array}$ \\
\hline
\end{tabular}

Comment: sealed envelopes may have been used without appropriate safeguards, e.g. not sequentially numbered, nor opaque

\begin{tabular}{|c|c|c|}
\hline $\begin{array}{l}\text { Blinding of participants } \\
\text { and personnel (perfor- } \\
\text { mance bias) } \\
\text { All outcomes }\end{array}$ & High risk & $\begin{array}{l}\text { Quote from publication: "Sealed envelopes, opened by study nurses", there- } \\
\text { fore personnel aware of allocation. The study was also unblinded "open ran- } \\
\text { domised controlled trial" }\end{array}$ \\
\hline
\end{tabular}

\begin{tabular}{|c|c|c|}
\hline $\begin{array}{l}\text { Blinding of outcome as- } \\
\text { sessment (detection bias) } \\
\text { All outcomes }\end{array}$ & Unclear risk & Comment: not described \\
\hline $\begin{array}{l}\text { Incomplete outcome data } \\
\text { (attrition bias) } \\
\text { All outcomes }\end{array}$ & Low risk & Comment: no missing outcome data \\
\hline $\begin{array}{l}\text { Selective reporting (re- } \\
\text { porting bias) }\end{array}$ & Low risk & $\begin{array}{l}\text { Comment: the study protocol is not available but it is clear that the published } \\
\text { reports include all expected outcomes, including those that were pre-specified }\end{array}$ \\
\hline Other bias & High risk & $\begin{array}{l}\text { Comment: data provided from only those who completed the study (rather } \\
\text { than all those initially randomised) - page } 207\end{array}$ \\
\hline
\end{tabular}

Gaskill 2009

\begin{tabular}{ll}
\hline Methods & $\begin{array}{l}\text { Cluster randomised controlled clinical trial } \\
\text { Randomisation ratio: } 1: 1 \\
\text { Superiority design }\end{array}$ \\
\hline Participants & 352 nursing home residents (245 female; 107 male); mean age 84.2 (SD 8.7) years \\
Inclusion criteria: not stated \\
Exclusion criteria: not stated \\
Diagnostic criteria: not stated \\
Nutrition education programme by nutrition coordinators compared with usual care \\
Number of trial centres: 8 \\
Treatment before trial: not stated
\end{tabular}


Gaskill 2009 (Continued)

Outcomes Outcomes reported in abstract of publication: nutritional status (SGA)

\begin{tabular}{ll}
\hline Study details & Run-in period: no \\
& Was trial terminated early: no \\
\hline Publication details & Language of publication: English \\
& Funding: non-commercial funding \\
& Publication status: peer review journal \\
\hline Stated aim for study & Quote from publication: "To investigate the impact of implementing a train-the-trainer nutrition pro- \\
\hline Notes & gramme on the nutritional status of older adults residing in residential care."
\end{tabular}

\section{Risk of bias}

\begin{tabular}{lll}
\hline Bias & Authors' judgement & Support for judgement \\
\hline $\begin{array}{l}\text { Random sequence genera- } \\
\text { tion (selection bias) }\end{array}$ & Unclear risk & $\begin{array}{l}\text { Quote from paper: "Four of the eight Residential Aged Care Facilities were se- } \\
\text { lected at random.." } \\
\text { Comment: method used not described }\end{array}$ \\
\hline $\begin{array}{l}\text { Allocation concealment } \\
\text { (selection bias) }\end{array}$ & Unclear risk & Comment: not described \\
\hline $\begin{array}{l}\text { Blinding of participants } \\
\text { and personnel (perfor- } \\
\text { mance bias) } \\
\text { All outcomes }\end{array}$ & Unclear risk & Comment: not described \\
\hline
\end{tabular}

\begin{tabular}{ll}
\hline Blinding of outcome as- & Unclear risk
\end{tabular}

All outcomes

Incomplete outcome data Unclear risk Comment: insufficient reporting of attrition data
(attrition bias)

All outcomes

\begin{tabular}{l}
$\begin{array}{l}\text { Selective reporting (re- } \\
\text { porting bias) }\end{array}$ \\
\hline Other bias \\
High risk
\end{tabular} $\begin{aligned} & \begin{array}{l}\text { Comment: baseline characteristics reported for whole group rather than for } \\
\text { intervention and control separately }\end{array} \\
& \text { Assessment of risk of bias in cluster-randomised trials } \\
& \begin{array}{l}\text { (1) Recruitment bias: unclear } \\
\text { (2) Baseline imbalance: number of diagnoses } \\
\text { (3) Loss of clusters: unclear } \\
\text { (4) Incorrect analysis: yes } \\
\text { (5) Comparability with individually randomised trials/different types of clus- } \\
\text { ters: unclear }\end{array}\end{aligned}$




\begin{tabular}{ll}
\hline Methods & Parallel randomised controlled clinical trial \\
& Randomisation ratio: $1: 1$ \\
& Superiority design \\
\hline Participants & 17 participants (10 female; 7 male); mean age 82.5 (SD 4.4) years intervention 84.6 (SD 3.8) years control \\
Inclusion criteria: $60-95$ years old; resident $>3$ months in the centre; unintentional weight loss $>7.5 \%$ \\
in previous 3 months or BMI $<24 \mathrm{~kg} / \mathrm{m}^{2}$ \\
Exclusion criteria: active cancer or chronic intestinal disease or terminally ill \\
Diagnostic criteria: dysphagia
\end{tabular}

Interventions Re-formed foods, thickened beverages and dietary supplements as necessary compared with traditional modified texture diet (control)

\section{Number of trial centres: 1}

Treatment before trial: not stated

\begin{tabular}{ll}
\hline Outcomes & Outcomes reported in abstract of publication: macro and micronutrient intake, weight and BMI \\
\hline Study details & Run-in period: no \\
& Was trial terminated early: no \\
\hline
\end{tabular}

\begin{tabular}{ll}
\hline Publication details & Language of publication: English \\
& Funding: not stated \\
& Publication status: peer review journal \\
\hline Stated aim for study & $\begin{array}{l}\text { Quote from publication: "To evaluate to nutrient intake of frail institutionalised elderly persons with } \\
\text { dysphagia, and to assess the impact of Sainte-Anne's Hospital Advanced Nutritional Care Programme, } \\
\text { on dietary intake and weight" }\end{array}$
\end{tabular}

\begin{tabular}{ll}
\hline Notes \\
\hline Risk of bias
\end{tabular}

\begin{tabular}{|c|c|c|}
\hline Bias & Authors' judgement & Support for judgement \\
\hline \multirow[t]{2}{*}{$\begin{array}{l}\text { Random sequence genera- } \\
\text { tion (selection bias) }\end{array}$} & Unclear risk & $\begin{array}{l}\text { Quote from paper: "Eligible subjects were randomly allocated....... using a } \\
\text { blocked allocation strategy." }\end{array}$ \\
\hline & & Comment: insufficient detail of method provided \\
\hline $\begin{array}{l}\text { Allocation concealment } \\
\text { (selection bias) }\end{array}$ & Low risk & $\begin{array}{l}\text { Quote from paper: "sealed opaque envelopes indicating subject assignment } \\
\text { were prepared off-site" }\end{array}$ \\
\hline $\begin{array}{l}\text { Blinding of participants } \\
\text { and personnel (perfor- } \\
\text { mance bias) } \\
\text { All outcomes }\end{array}$ & Unclear risk & Comment: not stated \\
\hline
\end{tabular}

Blinding of outcome as- Unclear risk Comment: not stated
sessment (detection bias) 
Germain 2006 (Continued)

All outcomes

Incomplete outcome data Low risk Comment: all participants fully accounted for
(attrition bias)

(attrition bias)

All outcomes

\begin{tabular}{lll}
\hline $\begin{array}{l}\text { Selective reporting (re- } \\
\text { porting bias) }\end{array}$ & Low risk & Comment: all outcomes reported \\
\hline Other bias & Low risk & Comment: groups comparable at baseline; data reported in table 1 and text \\
\hline
\end{tabular}

Hankey 1993

\begin{tabular}{ll}
\hline Methods & Parallel randomised controlled clinical trial \\
& Randomisation ratio: $1: 1$ \\
Superiority design
\end{tabular}

\begin{tabular}{ll}
\hline Participants & 20 frail elderly people; $\mathrm{N}=10$ intervention; $\mathrm{N}=10$ control \\
Inclusion criteria: frail elderly \\
Exclusion criteria: not stated \\
Diagnostic criteria: not stated
\end{tabular}

Interventions The intervention group received Build Up drink (1 unit) daily during routine drug prescription, in addition to their normal hospital diet. The usual care received the standard hospital diet

\section{Number of study centres: 1}

Treatment before trial: none described

\begin{tabular}{|c|c|}
\hline Outcomes & $\begin{array}{l}\text { Outcomes reported in abstract of publication: food intake, glucose polymer intake, anthropometric } \\
\text { measurements (TSF, MAMC) }\end{array}$ \\
\hline \multirow[t]{2}{*}{ Study details } & Run-in period: none \\
\hline & Was trial terminated early: no \\
\hline \multirow[t]{3}{*}{ Publication details } & Language of publication: English \\
\hline & Funding: not stated \\
\hline & Publicaton status: peer review journal \\
\hline Stated aim for study & $\begin{array}{l}\text { Quote from publication: "the effectiveness of dietary supplements for frail elderly subjects in continu- } \\
\text { ing care" }\end{array}$ \\
\hline Notes & - \\
\hline \multicolumn{2}{|l|}{ Risk of bias } \\
\hline Bias & Authors' judgement Support for judgement \\
\hline $\begin{array}{l}\text { Random sequence genera- } \\
\text { tion (selection bias) }\end{array}$ & $\begin{array}{l}\text { Quote from paper: "...subjects were randomised to control or supplemented } \\
\text { groups" }\end{array}$ \\
\hline
\end{tabular}


Hankey 1993 (Continued)

Comment: insufficient detail of method provided

\begin{tabular}{|c|c|c|}
\hline $\begin{array}{l}\text { Allocation concealment } \\
\text { (selection bias) }\end{array}$ & Unclear risk & Comment: not stated \\
\hline $\begin{array}{l}\text { Blinding of participants } \\
\text { and personnel (perfor- } \\
\text { mance bias) } \\
\text { All outcomes }\end{array}$ & Unclear risk & Comment: not stated \\
\hline $\begin{array}{l}\text { Blinding of outcome as- } \\
\text { sessment (detection bias) } \\
\text { All outcomes }\end{array}$ & Unclear risk & Comment: not stated \\
\hline $\begin{array}{l}\text { Incomplete outcome data } \\
\text { (attrition bias) } \\
\text { All outcomes }\end{array}$ & Low risk & Comment: no missing outcome data \\
\hline $\begin{array}{l}\text { Selective reporting (re- } \\
\text { porting bias) }\end{array}$ & Low risk & $\begin{array}{l}\text { Comment: the study protocol is not available but it is clear that the published } \\
\text { reports include all expected outcomes, including those that were pre-specified }\end{array}$ \\
\hline Other bias & Unclear risk & $\begin{array}{l}\text { Comment: insufficient information to assess whether an important risk of bias } \\
\text { exists }\end{array}$ \\
\hline
\end{tabular}

Hickson 2004

\begin{tabular}{ll}
\hline Methods & Parallel randomised controlled clinical trial \\
& Randomisation ratio: $1: 1$ \\
Superiority design
\end{tabular}

Participants Inclusion criteria: > 65 years old; admitted to medicine for the elderly wards

Exclusion criteria: unable to take food orally; not expected to survive the admission; planned discharge within 4 days; readmitted if already recruited into the trial

Diagnostic criteria: acutely ill with a range of clinical conditions

\begin{tabular}{ll}
\hline Interventions & Number of trial centres: 1 \\
& Treatment before trial: none \\
\hline Outcomes & $\begin{array}{l}\text { Outcomes reported in abstract of publication: nutritional status, mortality, length of stay, grip } \\
\text { strength, Barthel score, intravenous antibiotic prescription }\end{array}$ \\
\hline Study details & Run-in period: none \\
& Was trial terminated early: no \\
\hline Publication details & Language of publication: English \\
& Funding: non-commercial funding \\
& Publication status: peer review journal \\
\hline Stated aim for study & $\begin{array}{l}\text { Quote from publication: "to examine whether healthcare assistants trained to provide additional sup- } \\
\text { port with feeding will improve acutely ill elderly inpatients clinical outcomes" }\end{array}$
\end{tabular}


Hickson 2004 (Continued)

Notes

\section{Risk of bias}

\begin{tabular}{lll}
\hline Bias & Authors' judgement & Support for judgement \\
\hline $\begin{array}{l}\text { Random sequence genera- } \\
\text { tion (selection bias) }\end{array}$ & Low risk & $\begin{array}{l}\text { Quote from paper: stratified by ward and achieved using "computer generat- } \\
\text { ed random numbers tables" }\end{array}$ \\
\hline $\begin{array}{l}\text { Allocation concealment } \\
\text { (selection bias) }\end{array}$ & Low risk & $\begin{array}{l}\text { Quote from paper: "using sealed, opaque envelopes prepared by an indepen- } \\
\text { dent group" }\end{array}$ \\
\hline $\begin{array}{l}\text { Blinding of participants } \\
\text { and personnel (perfor- } \\
\text { mance bias) } \\
\text { All outcomes }\end{array}$ & Unclear risk & Comment: insufficient information \\
\hline
\end{tabular}

Blinding of outcome as- Unclear risk Comment: insufficient information

sessment (detection bias)

All outcomes

\begin{tabular}{lll}
\hline $\begin{array}{l}\text { Incomplete outcome data } \\
\text { (attrition bias) } \\
\text { All outcomes }\end{array}$ & Low risk & Comment: fully described \\
\hline $\begin{array}{l}\text { Selective reporting (re- } \\
\text { porting bias) }\end{array}$ & High risk & $\begin{array}{l}\text { Comment: no data reported on the following outcomes: use of services ques- } \\
\text { tionnaire, referral rate to therapists, readmission within } 6 \text { months, laxative } \\
\text { use, pressure sores, economic analysis }\end{array}$ \\
\hline Other bias & Low risk & $\begin{array}{l}\text { Comment: significantly more women in the intervention group otherwise both } \\
\text { groups comparable at baseline }\end{array}$ \\
\hline
\end{tabular}

Holyday 2012

\begin{tabular}{ll}
\hline Methods & Parallel randomised controlled clinical trial \\
& Randomisation ratio: $1: 1$ \\
& Superiority design \\
\hline Participants & 143 hospitalised patients (61 male: 82 female); age intervention 83.7 (SE 0.8) control 83.4 (SE 0.9) years \\
& $\begin{array}{l}\text { Inclusion criteria: all patients admitted under the care of a Geriatrician to an acute geriatric medicine } \\
\text { ward }\end{array}$
\end{tabular}

Exclusion criteria: expected length of stay $<72 \mathrm{~h}$; palliative care; unable to be nutritionally assessed; not speaking English; severe dementia or confusion; non-cooperation

Diagnostic criteria: acute geriatric medicine

\begin{tabular}{ll}
\hline Interventions & Malnutrition Care Pathway (modification of hospital meals; prescription of nutritional supplements or \\
snacks; flagging for feeding assistance; education of participants and carers; referral to other health \\
professionals and discharge planning) versus usual care
\end{tabular}

\section{Number of trial centres: 1}

Treatment before trial: not specified 
Holyday 2012 (Continued)

Outcomes

Outcomes reported in abstract of publication: length of hospital stay; readmissions; weight change; number of malnourished participants identified without routine nutrition screening

\begin{tabular}{ll}
\hline Study details & Run-in period: none \\
& Was trial terminated early: no \\
\hline Publication details & Language of publication: English \\
& $\begin{array}{l}\text { Funding: commercial and non-commercial funding - The Gut Foundation + Pharmatel Fresenius Kabi } \\
\text { PTY Ltd. }\end{array}$ \\
& Publication status: peer review journal \\
\hline Stated aim for study & $\begin{array}{l}\text { Quote from publication: "To examine the prevalence of malnutrition in acutely ill older patients and to } \\
\text { assess the impact of malnutrition screening and early dietetic intervention on weight, length of hospi- } \\
\text { tal stay, hospital costs and subsequent emergency presentations and hospital readmissions in geriatric } \\
\text { patients at risk of malnutrition using a randomised controlled trial" }\end{array}$
\end{tabular}

Notes

\section{Risk of bias}

\begin{tabular}{lll}
\hline Bias & Authors' judgement & Support for judgement \\
\hline $\begin{array}{l}\text { Random sequence genera- } \\
\text { tion (selection bias) }\end{array}$ & Low risk & $\begin{array}{l}\text { Quote from paper: "randomly allocated by computerised random number } \\
\text { generator" }\end{array}$ \\
\hline
\end{tabular}

Allocation concealment Unclear risk
(selection bias)

\begin{tabular}{|c|c|c|}
\hline $\begin{array}{l}\text { Blinding of participants } \\
\text { and personnel (perfor- } \\
\text { mance bias) } \\
\text { All outcomes }\end{array}$ & High risk & $\begin{array}{l}\text { Quote from publication: "not possible to blind the clinical dietitian to group } \\
\text { allocation" }\end{array}$ \\
\hline
\end{tabular}

Blinding of outcome as- High risk

Quote from publication: "as the outcomes are primarily objective measures sessment (detection bias) they are mostly not open to the influence of bias"

All outcomes

Incomplete outcome data Low risk Comment: all deaths and dropouts fully accounted for

(attrition bias)

All outcomes

Selective reporting (re- Low risk Comment: all outcomes fully reported
porting bias)

Other bias Low risk Comment: baseline characteristics similar between groups

Johansen 2004

$\begin{array}{ll}\text { Methods } & \text { Parallel randomised controlled clinical trial } \\ \text { Randomisation ratio: } 1: 1 \\ \text { Superiority design }\end{array}$


Johansen 2004 (Continued)

Participants

Inclusion criteria: NRS-2000 score > 3 on admission to hospital

Exclusion criteria: predicted admission < 4 days; < 18 years old; < 1 month expected survival; ability to understand Danish; previously included participants; patients next to another participant; pregnant and lactating; psychiatric disorder; haemodialysis; patients receiving or planned to receive EN or PN

Diagnostic criteria: varied

Interventions Received daily attention from the nutrition team (nurse and dietitian); motivation of participant and staff; daily monitoring and adjustment of nutrition care plan; secured supply of ordered food

Number of trial centres: 3 hospitals in Denmark

Treatment before trial: not described

Outcomes Outcomes reported in abstract of publication: length of stay; nutrition discharge index; health-related quality of life (Short Form -36 health survey)

\begin{tabular}{ll}
\hline Study details & Run-in period: no \\
& Was trial terminated early: no \\
\hline
\end{tabular}

Publication details Language of publication: English

Funding: non-commercial funding - Danish Ministry of Health + participating Hospitals

Publication status: peer review journal

Stated aim for study

Quote from publication: "To evaluate the clinical benefits of nutritional intervention in a random sample of all patients at nutritional risk according to Nutritional Risk Score -2002 from three different hospital levels"

\begin{tabular}{|c|c|c|}
\hline Notes & - & \\
\hline \multicolumn{3}{|l|}{ Risk of bias } \\
\hline Bias & Authors' judgement & Support for judgement \\
\hline $\begin{array}{l}\text { Random sequence genera- } \\
\text { tion (selection bias) }\end{array}$ & Low risk & $\begin{array}{l}\text { Quote from paper: participants selected "by a random numbers system" } \\
\text { Comment: suggests that random sequence appropriate }\end{array}$ \\
\hline $\begin{array}{l}\text { Allocation concealment } \\
\text { (selection bias) }\end{array}$ & Unclear risk & Comment: not described \\
\hline $\begin{array}{l}\text { Blinding of participants } \\
\text { and personnel (perfor- } \\
\text { mance bias) } \\
\text { All outcomes }\end{array}$ & Unclear risk & Comment: not described \\
\hline $\begin{array}{l}\text { Blinding of outcome as- } \\
\text { sessment (detection bias) } \\
\text { All outcomes }\end{array}$ & Unclear risk & $\begin{array}{l}\text { Comment: assessment of complications undertaken by a member of the in- } \\
\text { vestigation team blinded to allocation }\end{array}$ \\
\hline $\begin{array}{l}\text { Incomplete outcome data } \\
\text { (attrition bias) } \\
\text { All outcomes }\end{array}$ & Unclear risk & $\begin{array}{l}\text { Comment: clearly described in the results; intention-to-treat analysis under- } \\
\text { taken, however they do not report which group participants dropped out of }\end{array}$ \\
\hline
\end{tabular}


Johansen 2004 (Continued)

Selective reporting (re- Low risk Comment: all outcomes specified in the methods fully reported porting bias)

Other bias Low risk

Comment: baseline characteristics fully described; intervention and usual

cares comparable

Kraft 2012

Methods

Parallel randomised controlled clinical trial

Randomisation ratio: $1: 1$

Superiority design

Participants 26 participants; mean age 79.8 (SD 7.3) years; 10 male; 16 female

Inclusion criteria: weight loss $>10 \%$ in previous 6 months; $\mathrm{BMI}<21 \mathrm{~kg} / \mathrm{m}^{2}$; albumin $<35 \mathrm{~g} / \mathrm{L}$

Exclusion criteria: malignancy, dementia, liver cirrhosis, dialysis-dependent kidney insufficiency; insufficient cognitive ability; receiving professional care at home or living in a nursing home

Diagnostic criteria: malnourished on discharge from hospital

Interventions

Intervention group received an oral nutritional supplement and telemedicine monitoring comprising daily assessment of weight, compliance with supplement prescription and state of health. Responses triggered a range of nutritional management actions by a nurse employed by the tele-medicine centre

Number of trial centres: 1

Treatment before trial: not stated

Outcomes Outcomes reported in abstract of publication: weight and BMI

\begin{tabular}{ll}
\hline Study details & Run-in period: none \\
& Was trial terminated early: no
\end{tabular}

Publication details Language of publication: English

Funding: non-commercial and commercial funding - Ministry of Social Affairs and Health, Western Pomerania, Germany and Nutricia (Germany)

Publication status: peer review journal

Stated aim for study Quote from publication: "To evaluate the feasibility and explore the patients acceptance of the tele-
medical concept"

Notes -

\section{Risk of bias}

Bias Authors' judgement Support for judgement

Random sequence genera- Unclear risk tion (selection bias)

Quote from paper: "Patients were randomized consecutively into the intervention and control group"

Comment: insufficient details of the method provided 
Kraft 2012 (Continued)

\begin{tabular}{lll}
$\begin{array}{l}\text { Allocation concealment } \\
\text { (selection bias) }\end{array}$ & Unclear risk & Comment: not described \\
\hline $\begin{array}{l}\text { Blinding of participants } \\
\text { and personnel (perfor- } \\
\text { mance bias) }\end{array}$ & Unclear risk & Comment: not described \\
All outcomes &
\end{tabular}

\begin{tabular}{lll}
\hline $\begin{array}{l}\text { Blinding of outcome as- } \\
\text { sessment (detection bias) } \\
\text { All outcomes }\end{array}$ & Unclear risk & Comment: not described \\
\hline $\begin{array}{l}\text { Incomplete outcome data } \\
\begin{array}{l}\text { (attrition bias) } \\
\text { All outcomes }\end{array}\end{array}$ & High risk & $\begin{array}{l}\text { Comment: high risk because of high attrition rate in the intervention group i.e. } \\
\text { intervention }(\mathrm{N}=13) 8 \text { withdrew; control }(\mathrm{N}=13) 3 \text { withdrew; all withdrawals } \\
\text { accounted for }\end{array}$ \\
\hline
\end{tabular}

\begin{tabular}{lll}
\hline $\begin{array}{l}\text { Selective reporting (re- } \\
\text { porting bias) }\end{array}$ & Low risk & Comment: all outcome measures reported \\
\hline Other bias & Low risk & $\begin{array}{l}\text { Comment: baseline characteristics fully described; intervention and usual } \\
\text { cares comparable }\end{array}$ \\
\hline
\end{tabular}

Kretser 2003

$\begin{array}{ll}\text { Methods } & \text { Parallel randomised controlled clinical trial } \\ \text { Randomisation ratio: } 1: 1 \\ \text { Superiority design }\end{array}$

Participants 203 participants; 144 female: 59 male

Inclusion criteria: people on a waiting list or referred on hospital discharge for meals on wheels or responding to local advertisements

Exclusion criteria: MNA score > 22.5; self-reported terminal illness; medical conditions that precluded the meal being adequate or food allergy; previously received meals on wheels

Diagnostic criteria: not stated

21 meals and 14 snacks consisting of frozen meals, nutritional supplements and shelf-stable and frozen food items. Menus provided 100\% macro and micronutrient requirements for people over the age of 50 years. Daily phone call from older adult volunteer to provide safety and socialisation. Control = one hot meal five days a week at lunchtime

Number of trial centres: not relevant (all at home)

Treatment before trial: none

Outcomes Outcomes reported in abstract of publication: weight, MNA, functional status

\begin{tabular}{ll}
\hline Study details & Run-in period: none \\
& Was trial terminated early: no \\
\hline
\end{tabular}

Publication details Language of publication: English

Commercial/non-commercial/other funding: not stated 
Kretser 2003 (Continued)

Publication status: peer review journal

Stated aim for study Quote from publication: "To compare a traditional meals on wheels (MoW) programme consisting of one hot meal delivered daily, Monday through Friday, versus a new MoW programme consisting of 21 meals and 14 snacks that required some preparation delivered weekly"

\section{Notes}

\section{Risk of bias}

\begin{tabular}{|c|c|c|}
\hline Bias & Authors' judgement & Support for judgement \\
\hline \multirow[t]{2}{*}{$\begin{array}{l}\text { Random sequence genera- } \\
\text { tion (selection bias) }\end{array}$} & High risk & $\begin{array}{l}\text { Quote from publication: "Randomized treatment assignment was followed } \\
\text { with a few exceptions...Participants who were offered the new MoW model and } \\
\text { refused, were placed in the traditional MoW model" }\end{array}$ \\
\hline & & $\begin{array}{l}\text { Comment: insufficient detail of method provided as well as patients moving } \\
\text { between groups as above }\end{array}$ \\
\hline $\begin{array}{l}\text { Allocation concealment } \\
\text { (selection bias) }\end{array}$ & Unclear risk & Comment: not described \\
\hline $\begin{array}{l}\text { Blinding of participants } \\
\text { and personnel (perfor- } \\
\text { mance bias) } \\
\text { All outcomes }\end{array}$ & Unclear risk & Comment: not described \\
\hline
\end{tabular}

\begin{tabular}{lll}
\hline $\begin{array}{l}\text { Blinding of outcome as- } \\
\text { sessment (detection bias) } \\
\text { All outcomes }\end{array}$ & Unclear risk & Comment: not described \\
\hline $\begin{array}{l}\text { Incomplete outcome data } \\
\text { (attrition bias) } \\
\text { All outcomes }\end{array}$ & Unclear risk & $\begin{array}{l}\text { Comment: overall attrition reported but not from which groups they dropped } \\
\text { out }\end{array}$ \\
\hline $\begin{array}{l}\text { Selective reporting (re- } \\
\text { porting bias) }\end{array}$ & Low risk & Comment: all outcomes fully reported \\
\hline $\begin{array}{l}\text { Other bias } \\
\text { Unclear risk }\end{array}$ & $\begin{array}{l}\text { Comment: traditional MoW group had significantly lower functional ability (in- } \\
\text { strumental ADL) and lower education attainment }\end{array}$ \\
\hline
\end{tabular}

\section{Larsson 1990}

\begin{tabular}{ll}
\hline Methods & Parallel randomised controlled clinical trial \\
& Randomisation ratio: $1: 1$ \\
& Superiority design \\
\hline Participants & $\begin{array}{l}\text { Inclusion criteria: people with an expected hospital stay of more than } 3 \text { weeks, admitted consecutive } \\
\text { ly to a long-term medical care clinic }\end{array}$ \\
Exclusion criteria: not stated \\
Diagnostic criteria: diagnosis of participants included: malignancy, endocrine, neurological, heart, \\
vascular, respiratory, musculoskeletal, fracture
\end{tabular}


Larsson 1990 (Continued)

Interventions
Intervention group received nutritional supplementation (400 kcal) in the morning and afternoon between meals, when all patients on the ward were routinely supplied with drinks, as well as standard hospital diet. The usual care received standard hospital diet alone

Number of trial centres: 1

Treatment before trial: none described

Outcomes reported in abstract of publication: anthropometry, serum protein analysis, delayed hypersensitivity skin test, mobility, general physical condition, food intake

\begin{tabular}{ll} 
Study details & Run-in period: none \\
& Was trial terminated early: no \\
\hline Publication details & Language of publication: English \\
& $\begin{array}{l}\text { Funding: commercial/other funding - Swedish Medical Research Council, Research Fund of the County } \\
\text { of Ostergotland, Kabi Nutrition }\end{array}$
\end{tabular}

Publication status: peer review journal

Stated aim for study

Quote from publication: "to investigate the relationship between nutritional state and the development and healing of pressure sores in patients in a long term care clinic" (page 245). Larsson: "to evaluate the effect of dietary supplements on clinical outcome and nutritional status in a large group of geriatric patients" (Ek 1991)

\begin{tabular}{|c|c|c|}
\hline Notes & - & \\
\hline \multicolumn{3}{|l|}{ Risk of bias } \\
\hline Bias & Authors' judgement & Support for judgement \\
\hline \multirow[t]{2}{*}{$\begin{array}{l}\text { Random sequence genera- } \\
\text { tion (selection bias) }\end{array}$} & Unclear risk & $\begin{array}{l}\text { Quote from publication: "Randomisation was carried out by means of sealed } \\
\text { envelopes containing group designation". }\end{array}$ \\
\hline & & $\begin{array}{l}\text { Comment: insufficient information provided about the sequence generation } \\
\text { process }\end{array}$ \\
\hline $\begin{array}{l}\text { Allocation concealment } \\
\text { (selection bias) }\end{array}$ & Unclear risk & Comment: insufficient information to permit judgement \\
\hline $\begin{array}{l}\text { Blinding of participants } \\
\text { and personnel (perfor- } \\
\text { mance bias) } \\
\text { All outcomes }\end{array}$ & Unclear risk & Comment: insufficient information to permit judgement \\
\hline $\begin{array}{l}\text { Blinding of outcome as- } \\
\text { sessment (detection bias) } \\
\text { All outcomes }\end{array}$ & Unclear risk & Comment: insufficient information to permit judgement \\
\hline $\begin{array}{l}\text { Incomplete outcome data } \\
\text { (attrition bias) } \\
\text { All outcomes }\end{array}$ & Unclear risk & $\begin{array}{l}\text { Comment: number of study dropouts presented in figure but unclear which } \\
\text { group they belong to and the reason }\end{array}$ \\
\hline $\begin{array}{l}\text { Selective reporting (re- } \\
\text { porting bias) }\end{array}$ & Low risk & Comment: data not reported at all time points for all outcomes \\
\hline Other bias & High risk & $\begin{array}{l}\text { Comment: significant differences between groups at baseline in TSF and } \\
\text { weight index in men, and AMC in women were significantly lower in experi- }\end{array}$ \\
\hline
\end{tabular}




$\begin{array}{ll}\text { Methods } & \text { Cluster-randomised controlled trial } \\ & \text { Randomisation ratio: } 1: 1 \\ \text { Superiority design }\end{array}$

Participants 41 people living in residential care homes, 36 female, 5 male, mean age 91(SD 7) years

Inclusion criteria: $\mathrm{BMI}<18.5 \mathrm{kgm}^{2}$, without acute disease

Exclusion criteria: not described

Diagnostic criteria: mixed diagnoses, people living in residential care homes

\begin{tabular}{ll}
\hline Interventions & Provision of energy enriched meals vs usual care \\
& Number of trial centres: 21 residential care homes \\
& Treatment before trial: not described \\
\hline Outcomes & Outcomes reported in abstract of publication: energy intake, weight and BMI \\
\hline Study details & Run-in period: no \\
& Was trial terminated early: no \\
\hline Publication details & Language of publication: English \\
& Funding: commercial funding - GlaxoSmithKline \\
& Publication status: peer review journal \\
\hline Stated aim for study & Quote from publication: "To examine whether the nutritional status of aged undernourished residents \\
in care could be improved through dietary modification to increase energy intake but not portion size"
\end{tabular}

\section{Risk of bias}

\begin{tabular}{lll}
\hline Bias & Authors' judgement & Support for judgement \\
\hline $\begin{array}{l}\text { Random sequence genera- } \\
\text { tion (selection bias) }\end{array}$ & Unclear risk & $\begin{array}{l}\text { Quote from publication: "Random permuted block design, stratified by home } \\
\text { type (dementia/no dementia) by a statistician who had no contact with the } \\
\text { homes" } \\
\text { Comment: insufficient detail of method provided }\end{array}$ \\
\hline $\begin{array}{l}\text { Allocation concealment } \\
\text { (selection bias) }\end{array}$ & Low risk & $\begin{array}{l}\text { Quote from publication: "Allocation made post recruitment and baseline } \\
\text { screening by a statistician who had no contact with the homes" }\end{array}$ \\
$\begin{array}{l}\text { Blinding of participants } \\
\text { and personnel (perfor- } \\
\text { mance bias) }\end{array}$ & Unclear risk & $\begin{array}{l}\text { Comment: not mentioned. As energy enrichment was of usual meals it would } \\
\text { have been possible to blind participants to the intervention }\end{array}$ \\
\hline
\end{tabular}


Leslie 2012 (Continued)

Blinding of outcome as- Unclear risk Comment: not mentioned. Assessment of weight and food intake might have sessment (detection bias) been influenced by knowing the study group

All outcomes

$\begin{array}{ll}\text { Incomplete outcome data Low risk } & \begin{array}{l}\text { Comment: the number of participants that dropped out and the reasons are } \\ \text { (attrition bias) }\end{array}\end{array}$

All outcomes

given

Selective reporting (re- Low risk Comment: all specified outcomes are reported

porting bias)

Other bias High risk Assessment of risk of bias in cluster-randomised trials

(1) Recruitment bias: no

(2) Baseline imbalance: unclear

(3) Loss of clusters: unclear

(4) Incorrect analysis: no

(5) Comparability with individually randomised trials/different types of clus-

ters: different types of clusters

Lin 2010

Methods Cluster-randomised controlled clinical trial

Randomisation ratio: $1: 1: 1$

Superiority design

Participants Inclusion criteria: diagnosis of dementia; EdFed score $\geq 2$; able to stay in institution for duration of study; Mini Mental State Examination score 10-23

Exclusion criteria: not described

Diagnostic criteria: diagnosis of dementia

\begin{tabular}{ll}
\hline Interventions & Number of trial centres: 3 \\
& Treatment before trial: not described
\end{tabular}
\begin{tabular}{ll}
\hline Outcomes & $\begin{array}{l}\text { Outcomes reported in abstract of publication: EdFed score; frequency of physical and verbal assis- } \\
\text { tance provided; nutritional status }\end{array}$
\end{tabular}

\begin{tabular}{ll}
\hline Study details & Run-in period: no \\
& Was trial terminated early: no \\
\hline Publication details & Language of publication: English \\
& Funding: non-commercial funding - National Health Research Insitute \\
& Publication status: peer review journal \\
\hline Stated aim for study & Quote from publication: "To investigate the effectiveness of training of spaced-retrieval and Montes- \\
& sori-based activities in decreasing feeding difficulty and nutritional status for residents with dementia" \\
\hline
\end{tabular}


Lin 2010 (Continued)

Notes

\section{Risk of bias}

\begin{tabular}{lll}
\hline Bias & Authors' judgement & Support for judgement \\
\hline $\begin{array}{l}\text { Random sequence genera- } \\
\text { tion (selection bias) }\end{array}$ & Unclear risk & $\begin{array}{l}\text { Quote from paper: "To avoid confounding, the three institutes were randomly } \\
\text { assigned ...." }\end{array}$ \\
& & Comment: insufficient detail of method provided
\end{tabular}

\begin{tabular}{ll}
\hline $\begin{array}{l}\text { Allocation concealment } \\
\text { (selection bias) }\end{array}$ & Unclear risk \\
\hline
\end{tabular}

Blinding of participants $\quad$ Unclear risk Comment: not described
and personnel (perfor-
mance bias)
All outcomes

Blinding of outcome as- Low risk sessment (detection bias)

All outcomes
Quote from publication: "The data collectors did not know which group the subjects belonged to".

\begin{tabular}{|c|c|c|}
\hline $\begin{array}{l}\text { Incomplete outcome data } \\
\text { (attrition bias) } \\
\text { All outcomes }\end{array}$ & Unclear risk & $\begin{array}{l}\text { Comment: state reason for dropouts, but unclear which groups they dropped } \\
\text { out of }\end{array}$ \\
\hline
\end{tabular}

\begin{tabular}{|c|c|c|}
\hline $\begin{array}{l}\text { Selective reporting (re- } \\
\text { porting bias) }\end{array}$ & Low risk & Comment: all outcomes fully reported \\
\hline \multirow[t]{7}{*}{ Other bias } & High risk & $\begin{array}{l}\text { Comment: baseline characteristics reported; significant difference in ADL ob- } \\
\text { served. }\end{array}$ \\
\hline & & Assessment of risk of bias in cluster-randomised trials \\
\hline & & (1) Recruitment bias: no \\
\hline & & (2) Baseline imbalance: frail status \\
\hline & & (3) Loss of clusters: no \\
\hline & & (4) Incorrect analysis: no \\
\hline & & $\begin{array}{l}\text { (5) Comparability with individually randomised trials/different types of clus- } \\
\text { ters: different types of clusters }\end{array}$ \\
\hline
\end{tabular}

\section{Lin 2011}

\begin{tabular}{ll}
\hline Methods & Cluster- and cross-over randomised controlled clinical trial \\
& Randomisation ratio: $1: 1$ \\
& Superiority design \\
\hline Participants & $\begin{array}{l}29 \text { participants; mean age } 82.9 \text { (SD 6.0) years; } 17 \text { male: } 12 \text { female with dementia in care home. Appear } \\
\text { to be identical to participants in Group } 2 \text { in Lin } 2010 ; \text { No response from study author }\end{array}$ \\
& $\begin{array}{l}\text { Inclusion criteria: diagnosis of dementia ; } \geq 2 \text { Edinburgh Feeding Evaluation in Dementia scale } \\
\text { (EdFed); MMSE score }=10-23\end{array}$
\end{tabular}


Lin 2011 (Continued)

Exclusion criteria: not stated

Diagnostic criteria: dementia

Interventions Montessori intervention including sensory stimulation, procedural movements (e.g. hand eye co-ordi-
nation) and extension and conclusion activities

Number of trial centres: 2

Treatment before trial: none

Outcomes Outcomes reported in abstract of publication: EdFed score; Eating Behaviours score; MNA score; self-
feeding frequency and self-feeding time

Study details Run-in period: 2-week wash out between cross-over

Was study terminated early: no

Publication details Language of publication: English

Funding: non-commercial funding - National Health Research Inistitute (Taiwan)

Publication status: peer review journal

\begin{tabular}{ll}
\hline Stated aim for study & $\begin{array}{l}\text { Quote from publication: "To investigate the efficacy of a Montessori intervention in improving the eat- } \\
\text { ing ability and nutritional status of residents with dementia in long term care facilities" }\end{array}$ \\
\hline Notes & - \\
\hline
\end{tabular}

\section{Risk of bias}

\begin{tabular}{|c|c|c|}
\hline Bias & Authors' judgement & Support for judgement \\
\hline \multirow[t]{2}{*}{$\begin{array}{l}\text { Random sequence genera- } \\
\text { tion (selection bias) }\end{array}$} & Unclear risk & $\begin{array}{l}\text { Quote from paper: "To avoid contamination among participants ......the two } \\
\text { demential special care units were randomly assigned....." }\end{array}$ \\
\hline & & Comment: insufficient information provided to permit judgement \\
\hline $\begin{array}{l}\text { Allocation concealment } \\
\text { (selection bias) }\end{array}$ & Unclear risk & Comment: not described \\
\hline $\begin{array}{l}\text { Blinding of participants } \\
\text { and personnel (perfor- } \\
\text { mance bias) } \\
\text { All outcomes }\end{array}$ & Unclear risk & $\begin{array}{l}\text { Comment: described as not blinded, lack of blinding therefore may have influ- } \\
\text { enced participant responses }\end{array}$ \\
\hline
\end{tabular}

\begin{tabular}{ll} 
Blinding of outcome as- & Low risk \\
sessment (detection bias) & \\
All outcomes & \\
\hline
\end{tabular}

Incomplete outcome data Unclear risk Comment: not described

(attrition bias)

All outcomes

\begin{tabular}{lll}
\hline $\begin{array}{l}\text { Selective reporting (re- } \\
\text { porting bias) }\end{array}$ & Low risk & Comment: all outcomes fully reported \\
\hline Other bias & High risk & $\begin{array}{l}\text { Comment: baseline data suggest considerable variation in length of institu- } \\
\text { tionalisation and length of time diagnosed with dementia }\end{array}$
\end{tabular}


Lin 2011 (Continued)

\section{Assessment of risk of bias in cluster-randomised trials}

(1) Recruitment bias: no

(2) Baseline imbalance: frail status

(3) Loss of clusters: no

(4) Incorrect analysis: no

(5) Comparability with individually randomised trials/different types of clusters: different types of clusters

\section{Mathey 2001a}

\begin{tabular}{|c|c|c|}
\hline Methods & \multicolumn{2}{|c|}{$\begin{array}{l}\text { Cluster randomised controlled clinical trial } \\
\text { Randomisation ratio: } 1: 1 \\
\text { Superiority design }\end{array}$} \\
\hline Participants & \multicolumn{2}{|c|}{$\begin{array}{l}\text { Inclusion criteria: > } 65 \text { years old; resident in nursing home for }>3 \text { months } \\
\text { Exclusion criteria: parenteral nutrition; terminal phase of disease; severe anaemia } \\
\text { Diagnostic criteria: varied }\end{array}$} \\
\hline Interventions & \multicolumn{2}{|c|}{$\begin{array}{l}\text { Number of trial centres: } 1 \\
\text { Treatment before trial: not stated }\end{array}$} \\
\hline Outcomes & \multicolumn{2}{|c|}{$\begin{array}{l}\text { Outcomes reported in abstract of publication: weight; dietary intake; biochemical indicators; health- } \\
\text { related quality of life (SIP); life satisfaction score (PGCMS) }\end{array}$} \\
\hline Study details & \multicolumn{2}{|c|}{$\begin{array}{l}\text { Run-in period: no } \\
\text { Was trial terminated early: no }\end{array}$} \\
\hline Publication details & \multicolumn{2}{|c|}{$\begin{array}{l}\text { Language of publication: English } \\
\text { Funding: not stated } \\
\text { Publication status: peer review journal }\end{array}$} \\
\hline Stated aim for study & \multicolumn{2}{|c|}{$\begin{array}{l}\text { Quote from publication: "To determine the effect of an improved ambience of food consumption on } \\
\text { health and nutritional status of Dutch nursing home elderly residents" }\end{array}$} \\
\hline Notes & \multicolumn{2}{|l|}{-} \\
\hline Risk of bias & & \\
\hline Bias & Authors' judgement & Support for judgement \\
\hline $\begin{array}{l}\text { Random sequence genera- } \\
\text { tion (selection bias) }\end{array}$ & Unclear risk & $\begin{array}{l}\text { Quote from paper: "Four wards, each with } 15 \text { residents and comparable for } \\
\text { diseases and treatment were randomly assigned to be in either the control } \\
\text { (two wards) or the experimental group (two wards)." } \\
\text { Comment: insufficient detail of method provided }\end{array}$ \\
\hline
\end{tabular}


Mathey 2001a (Continued)

Allocation concealment Unclear risk Comment: no details provided (selection bias)

Blinding of participants Unclear risk Comment: not stated and personnel (performance bias)

All outcomes

Blinding of outcome as- Unclear risk Comment: not stated

sessment (detection bias)

All outcomes

Incomplete outcome data Low risk Comment: attrition fully reported

(attrition bias)

All outcomes

Selective reporting (re- Low risk Comment: all outcomes fully reported
porting bias)

Other bias High risk

Comment: baseline characteristics fully reported (including dropouts); control and intervention groups comparable at baseline

\author{
Assessment of risk of bias in cluster-randomised trials \\ (1) Recruitment bias: no \\ (2) Baseline imbalance: frail status \\ (3) Loss of clusters: no \\ (4) Incorrect analysis: no \\ (5) Comparability with individually randomised trials/different types of clus- \\ ters: different types of clusters
}

\begin{tabular}{ll} 
Methods & Parallel randomised controlled clinical trial \\
& Randomisation ratio: $1: 1$ \\
& Superiority design \\
\hline Participants & $\begin{array}{l}67 \text { elderly care home residents; mean age intervention } 84.6 \text { (SD } 6.1) \text { years; control } 83 \text { (SD 5.5) years; } 54 \\
\text { female: } 13 \text { male }\end{array}$ \\
& $\begin{array}{l}\text { Inclusion criteria: }>65 \text { years, resided in care home }>3 \text { months and consuming cooked meal provided } \\
\text { by care home kitchen at least } 5 \text { days/week }\end{array}$
\end{tabular}

Exclusion criteria: dementia, hospitalised, depression; in terminal phase; allergy to MSG

Diagnostic criteria: not specified

$\begin{array}{ll}\text { Interventions } & \begin{array}{l}\text { Four flavour powders to enhance the cooked meal (chicken, beef, turkey or lemon) using } 1( \pm 0.2) \mathrm{g} \\ \text { flavour powder }\end{array}\end{array}$

\title{
Number of trial centres: 1
}

Treatment before trial: not stated 
Mathey 2001b (Continued)

Outcomes Outcomes reported in abstract of publication: body weight, energy intake and hunger

\begin{tabular}{ll}
\hline Study details & Run-in period: one \\
& Was trial terminated early: no \\
\hline Publication details & $\begin{array}{l}\text { Language of publication: English } \\
\text { Funding: commercial funding - flavours donated by IFF BV; funding from Friesland Coberco Research } \\
\text { and the Suikerstichting }\end{array}$ \\
& Publication status: peer review journal \\
\hline Stated aim for study & $\begin{array}{l}\text { Quote from publication: "To determine whether the addition of flavour enhancers to the cooked meals } \\
\text { over } 16 \text { weeks would lead to an increase in food consumption and thereby provide nutritional benefits } \\
\text { to elderly nursing home residents" }\end{array}$
\end{tabular}

Notes

\section{Risk of bias}

Bias Authors' judgement Support for judgement

Random sequence genera- Unclear risk tion (selection bias)
Quote from paper: "subjects were randomly assigned to be in the control group ..or the flavour group.."

Comment: insufficient detail of the method provided

\begin{tabular}{lll}
\hline $\begin{array}{l}\text { Allocation concealment } \\
\text { (selection bias) }\end{array}$ & Unclear risk & Comment: not described \\
\hline $\begin{array}{l}\text { Blinding of participants } \\
\text { and personnel (perfor- }\end{array}$ & Unclear risk & Comment: not described \\
mance bias) & & \\
All outcomes & & \\
\hline
\end{tabular}

\begin{tabular}{lll}
\hline $\begin{array}{l}\text { Blinding of outcome as- } \\
\text { sessment (detection bias) } \\
\text { All outcomes }\end{array}$ & Unclear risk & Comment: not described \\
\hline $\begin{array}{l}\text { Incomplete outcome data } \\
\text { (attrition bias) } \\
\text { All outcomes }\end{array}$ & Low risk & Comment: all dropouts fully accounted for \\
\hline $\begin{array}{l}\text { Selective reporting (re- } \\
\text { porting bias) }\end{array}$ & Low risk & Comment: all outcomes specified in the methods fully reported \\
\hline $\begin{array}{l}\text { Other bias } \\
\text { Low risk }\end{array}$ & $\begin{array}{l}\text { Comment: baseline characteristics fully reported; control and intervention } \\
\text { groups comparable at baseline }\end{array}$ \\
\hline
\end{tabular}

Munk 2014

\begin{tabular}{ll}
\hline Methods & Parallel randomised controlled clinical trial \\
& Randomisation ratio: $1: 1$ \\
Superiority design
\end{tabular}


Munk 2014 (Continued)

Participants

84 people newly admitted to hospital; mean age intervention 75 (SD 10) years; control 74 (SD 11) years; 47 female, 34 male (data on those that completed the study)

Inclusion criteria:new admissions to hospital, $\geq 18$ years old and at nutritional risk according to NRS-2002, able to eat orally, anticipated length of stay > 3 days, sufficient language proficiency

Exclusion criteria: dysphagia, food allergy or intolerance, anatomical obstruction preventing food intake, receiving enteral or parenteral nutrition, judged to be terminally ill

Diagnostic criteria: admitted to oncology, orthopaedics or urology wards

Interventions
An a la carte menu of small dishes enriched with natural energy-dense ingredients and supplemented with protein powder

\section{Number of trial centres: 1}

Treatment before trial: not stated

\section{Outcomes}

Outcomes reported in abstract of publication: percent reaching their calculated energy and protein requirements, mean energy and protein intake, body weight, handgrip strength, LOS, mortality

\begin{tabular}{ll}
\hline Study details & Run-in period: 5 weeks to ensure optimal staff training. Recruitment started at the end of the run-in \\
Was trial terminated early: no
\end{tabular}

Publication details Language of publication: English

Funding: commercial funding - protein powder donated by Toft Care System, Copenhagen, Denmark. Study funded by Herlev University Hospital Research Unit

Publication status: peer review journal

\begin{tabular}{ll}
\hline Stated aim for study & Quote "to investigate whether a novel food service concept with protein supplementation would in- \\
crease protein and energy intake in hospitalised patients at nutritional risk".
\end{tabular}

$$
\text { Notes }
$$

\section{Risk of bias}

\begin{tabular}{lll}
\hline Bias & Authors' judgement & Support for judgement \\
\hline $\begin{array}{l}\text { Random sequence genera- } \\
\text { tion (selection bias) }\end{array}$ & Low risk & $\begin{array}{l}\text { Quote from paper: "The allocation sequence was generated by a secretary } \\
\text { who was not otherwise involved in the trial" }\end{array}$ \\
\hline $\begin{array}{l}\text { Allocation concealment } \\
\text { (selection bias) }\end{array}$ & Low risk & $\begin{array}{l}\text { Quote from paper: "using sealed opaque envelopes, with a total of } 9 \text { blocks } \\
\text { each with } 10 \text { envelopes" }\end{array}$ \\
\hline $\begin{array}{l}\text { Blinding of participants } \\
\text { and personnel (perfor- } \\
\text { mance bias) } \\
\text { All outcomes }\end{array}$ & High risk & $\begin{array}{l}\text { Comment: participants and study personnel were not blinded to group alloca- } \\
\text { tion. Blinding of participants would not be possible due to the nature of the in- } \\
\text { tervention }\end{array}$ \\
\hline
\end{tabular}
sessment (detection bias) All outcomes
Blinding of outcome as- High risk
Comment: data assessors were not blinded to group allocation. Blinding of the assessors was judged by the authors to be difficult as participants were likely to reveal their group allocation. The analyses were conducted blinded to group allocation

$\begin{array}{ll}\begin{array}{l}\text { Incomplete outcome data } \\ \text { (attrition bias) }\end{array} & \text { Low risk } \\ \text { All outcomes } & \begin{array}{l}\text { Comment: three participants did not receive the intervention and so not in- } \\ \text { cluded in the study }\end{array}\end{array}$
cluded in the study
Comment: three participants did not receive the intervention and so not in- 
Munk 2014 (Continued)

Selective reporting (re- Low risk Comment: all outcomes specified in the methods reported
porting bias)

\begin{tabular}{|c|c|c|}
\hline Other bias & Low risk & $\begin{array}{l}\text { Comment: baseline characteristics fully reported; control and intervention } \\
\text { groups comparable at baseline }\end{array}$ \\
\hline
\end{tabular}

Nijs 2006

\begin{tabular}{ll}
\hline Methods & Cluster-randomised controlled clinical trial \\
& Randomisation ratio: $1: 1$ \\
& Superiority design \\
\hline Participants & $\begin{array}{l}\text { Inclusion criteria: nursing homes: medium sized, with a general population, two wards for people with } \\
\text { chronic somatic diseases, long-term or permanent stay, located in different parts of the country, simi- } \\
\text { lar for staff numbers, disciplines, education levels of carers, newness of infrastructure, location and res- } \\
\text { idents' activities }\end{array}$
\end{tabular}

Exclusion criteria: not stated

Diagnostic criteria: not stated

\begin{tabular}{ll}
\hline Interventions & $\begin{array}{l}\text { Number of trial centres: } 5 \\
\text { Treatment before trial: none }\end{array}$ \\
\hline Outcomes & Outcomes reported in abstract of publication: dietary intake, MNA score \\
\hline Study details & Run-in period: 2 -month run-in to allow nurses to accommodate the change in organisation \\
& Was trial terminated early: no \\
\hline Publication details & Language of publication: English \\
& $\begin{array}{l}\text { Funding: non-commercial funding } \\
\text { Publication status: peer review journal }\end{array}$ \\
\hline Stated aim for study & $\begin{array}{l}\text { Quote from publication: "to investigate the effect of family-style meals on energy intake and the risk of } \\
\text { malnutrition in Dutch nursing home residents" }\end{array}$
\end{tabular}

\begin{tabular}{|c|c|c|}
\hline Notes & - & \\
\hline \multicolumn{3}{|l|}{ Risk of bias } \\
\hline Bias & Authors' judgement & Support for judgement \\
\hline \multirow[t]{2}{*}{$\begin{array}{l}\text { Random sequence genera- } \\
\text { tion (selection bias) }\end{array}$} & High risk & $\begin{array}{l}\text { Quote from paper: "The wards' name with the initial letter occurring first in } \\
\text { the alphabet became the intervention ward". }\end{array}$ \\
\hline & & $\begin{array}{l}\text { Comment: the randomisation was based on the ward name and therefore pre- } \\
\text { dictable }\end{array}$ \\
\hline $\begin{array}{l}\text { Allocation concealment } \\
\text { (selection bias) }\end{array}$ & High risk & Comment: no concealment \\
\hline
\end{tabular}


Nijs 2006 (Continued)

Blinding of participants Unclear risk Comment: not done, but probably not possible to do and personnel (performance bias)

All outcomes

\begin{tabular}{|c|c|c|}
\hline $\begin{array}{l}\text { Blinding of outcome as- } \\
\text { sessment (detection bias) } \\
\text { All outcomes }\end{array}$ & Unclear risk & Comment: not stated \\
\hline $\begin{array}{l}\text { Incomplete outcome data } \\
\text { (attrition bias) } \\
\text { All outcomes }\end{array}$ & Low risk & Comment: all participants are fully accounted for \\
\hline $\begin{array}{l}\text { Selective reporting (re- } \\
\text { porting bias) }\end{array}$ & Low risk & Comment: all outcomes reported \\
\hline Other bias & High risk & $\begin{array}{l}\text { Assessment of risk of bias in cluster-randomised trials } \\
\text { (1) Recruitment bias: unclear } \\
\text { (2) Baseline imbalance: age, sex } \\
\text { (3) Loss of clusters: unclear } \\
\text { (4) Incorrect analysis: no } \\
\text { (5) Comparability with individually randomised trials / different types of clus- } \\
\text { ters: unclear }\end{array}$ \\
\hline
\end{tabular}

Olofsson 2007

\begin{tabular}{ll}
\hline Methods & Parallel randomised controlled clinical trial \\
& Randomisation ratio: $1: 1$ \\
& Superiority design \\
\hline Participants & Inclusion criteria: femoral neck fracture; > 70 years old; admitted to orthopaedic wards \\
& Exclusion criteria: severe rheumatoid arthritis, hip osteoarthritis or renal failure or metastatic fracture \\
and bed-ridden before the injury & Diagnostic criteria: femoral neck fracture \\
& Number of trial centres: 1 \\
Interventions & $\begin{array}{l}\text { Treatment before trial: none } \\
\text { Complex intervention: staff education; team work, individual care planning; prevention and treat- } \\
\text { ment of delirium and complications; nutrition; rehabilitation; secondary prevention of falls and frac- } \\
\text { tures; osteoporosis prophylaxis }\end{array}$
\end{tabular}
Outcomes
Outcomes reported in abstract of publication: days of delirium; decubitus ulcers; length of stay; BMI, body weight; MNA score

\begin{tabular}{ll}
\hline Study details & Run-in period: none \\
Was trial terminated early: no
\end{tabular}

Publication details Language of publication: English

Supportive interventions for enhancing dietary intake in malnourished or nutritionally at-risk adults (Review) 
Funding: non-commercial funding - Borgerskapt in Umea Research Foundation; the Dementia Fund; the Vardal foundation; the Joint committee of the Northern Health Region of Sweden; the JC Kempe Memorial Foundation; the Foundation for the Medical Faculty, University of Umea, local councils and the Swedish Research Council

Publication status: peer review journal

Stated aim for study

Quote from publication: "To investigate whether a nutritional intervention which was part of a multi-factorial intervention programme for old women and men with a femoral neck fracture had any effect on post-operative complications during hospitalisation and on nutritional status at four months follow-up"

Notes -

\section{Risk of bias}

Bias Authors' judgement Support for judgement

Random sequence genera- Unclear risk tion (selection bias)

Quote from paper: "Patients were randomised to post op care in a geriatric ward with a special intervention programme or to conventional care in the orthopaedic department. All participants received an envelope while in the emergency room, but it was not opened until immediately before surgery...."

Comment: insufficient detail of method provided

\begin{tabular}{lll}
\hline $\begin{array}{l}\text { Allocation concealment } \\
\text { (selection bias) }\end{array}$ & Low risk & $\begin{array}{l}\text { Comment: "sealed opaque envelopes stratified by operation"; envelopes } \\
\text { opened by a nurse not involved in the study }\end{array}$ \\
\hline $\begin{array}{l}\text { Blinding of participants } \\
\text { and personnel (perfor- } \\
\text { mance bias) } \\
\text { All outcomes }\end{array}$ & High risk & $\begin{array}{l}\text { Comment: not blinded; staff on the control ward knew that a new programme } \\
\text { was being implemented on another ward in the hospital }\end{array}$ \\
\hline $\begin{array}{l}\text { Blinding of outcome as- } \\
\text { sessment (detection bias) } \\
\text { All outcomes }\end{array}$ & Low risk & $\begin{array}{l}\text { Comment: assessments on the intervention ward were carried out by a nurse } \\
\text { on the control ward and vice versa. A specialist in geriatric medicine who was } \\
\text { not working in either of the two departments, and did not know which groups } \\
\text { the patients were randomised to, analysed all the outcomes }\end{array}$ \\
\hline
\end{tabular}

Incomplete outcome data Low risk Comment: fully described

(attrition bias)

All outcomes

\begin{tabular}{lll}
\hline $\begin{array}{l}\text { Selective reporting (re- } \\
\text { porting bias) }\end{array}$ & Low risk & Comment: all outcomes fully reported \\
\hline Other bias & Unclear risk & $\begin{array}{l}\text { Comment: baseline characteristics reported; groups comparable apart from } \\
\text { prevalence of heart failure }\end{array}$ \\
\hline
\end{tabular}

\section{Pivi 2011}

Methods Parallel randomised controlled clinical trial

Randomisation ratio: $1: 1: 1$

Superiority design 
Pivi 2011 (Continued)

Exclusion criteria: other forms of dementia; receiving tube feeding; diabetes or renal disease

Diagnostic criteria: Alzheimer's disease

\begin{tabular}{ll}
\hline Interventions & Number of trial centres: 1 \\
& Treatment before trial: no \\
\hline Outcomes & $\begin{array}{l}\text { Outcomes reported in abstract of publication: weight; BMI; MAC and ;MAMC; TSF; total protein; total } \\
\text { lymphocyte count }\end{array}$
\end{tabular}

lymphocyte count

\begin{tabular}{ll}
\hline Study details & Run-in period: none \\
Was trial terminated early: no
\end{tabular}

\begin{tabular}{ll}
\hline Publication details & Language of publication: English \\
& Funding: commercial/non-commercial funding - Ministry of Education; Abbott Laboratories \\
& Publication status: peer review journal \\
\hline Stated aim for study & $\begin{array}{l}\text { Quote from publication: "To determine if there is any difference between oral nutritional supplementa- } \\
\text { tion and nutrition education on the nutritional status of patients with AD" }\end{array}$ \\
\hline Notes & -
\end{tabular}

\section{Risk of bias}

\begin{tabular}{lll}
\hline Bias & Authors' judgement & Support for judgement \\
\hline $\begin{array}{l}\text { Random sequence genera- } \\
\text { tion (selection bias) }\end{array}$ & Unclear risk & $\begin{array}{l}\text { Quote from paper: "subjects were randomised into three groups....." } \\
\text { Comment: insufficient detail of method provided }\end{array}$ \\
\hline $\begin{array}{l}\text { Allocation concealment } \\
\text { (selection bias) }\end{array}$ & Unclear risk & Comment: not described \\
\hline $\begin{array}{l}\text { Blinding of participants } \\
\text { and personnel (perfor- } \\
\text { mance bias) } \\
\text { All outcomes }\end{array}$ & Unclear risk & Comment: not described \\
\hline
\end{tabular}

Blinding of outcome as- Unclear risk Comment: not described
sessment (detection bias)

All outcomes

Incomplete outcome data Low risk Comment: fully described
(attrition bias)

All outcomes

Selective reporting (re- Low risk $\quad$ Comment: fully reported
porting bias)

Other bias Unclear risk Comment: baseline characteristics reported; groups comparable

Methods Parallel randomised controlled clinical trial


Potter 2001 (Continued)

\section{Randomisation ratio: $1: 1$}

\section{Superiority design}

Participants Inclusion criteria: emergency admissions to medicine for the elderly unit (aged over 60), emergency admissions from home, ability to gain consent from participants or relatives, no known malignancy, ability to swallow, non obesity, BMI < 75th percentile.

Exclusion criteria: overweight (BMI > 75th percentile), in terminal stage of illness, or had swallow difficulty preventing oral intake

Diagnostic criteria: unwell elderly people

Interventions Intervention group received $120 \mathrm{~mL}$ sip feed $3 \mathrm{x}$ daily throughout hospitalisation. The usual care received normal ward diet

Number of trial centres: 1 - medicine for the elderly unit in a Scottish Hospital

Treatment before trial: none

Outcomes Outcomes reported in abstract of publication: anthropometry, mortality, length of hospital stay, functional recovery, rates of institutionalisation, patient compliance with supplement, total energy intake, nursing staff views of the method

\begin{tabular}{ll}
\hline Study details & $\begin{array}{l}\text { Run-in period: no } \\
\text { Was trial terminated early: no }\end{array}$ \\
\hline Publication details & Language of publication: English \\
& Funding: commercial/other funding - Chief Scientist's Office of Scottish Office, and Frusenius UK \\
& Publication status: peer review journal \\
\hline Stated aim for study & $\begin{array}{l}\text { Quote from publication: "to assess whether prescription of oral sip-feed supplements in small quanti- } \\
\text { ties in the medicine prescription chart and distribution at medication rounds could increase total ener- } \\
\text { gy intake and provide sufficient energy to prevent nutritional decline" (Roberts 2003) }\end{array}$
\end{tabular}

Notes

\section{Risk of bias}

\begin{tabular}{|c|c|c|}
\hline Bias & Authors' judgement & Support for judgement \\
\hline \multirow[t]{2}{*}{$\begin{array}{l}\text { Random sequence genera- } \\
\text { tion (selection bias) }\end{array}$} & Unclear risk & $\begin{array}{l}\text { Quote from paper: "Patients were assigned to the intervention arm random- } \\
\text { ly..." }\end{array}$ \\
\hline & & Comment: not described in sufficient detail \\
\hline \multirow[t]{2}{*}{$\begin{array}{l}\text { Allocation concealment } \\
\text { (selection bias) }\end{array}$} & Unclear risk & $\begin{array}{l}\text { Quote from paper: "using sealed envelopes containing allocation specifica- } \\
\text { tion" }\end{array}$ \\
\hline & & $\begin{array}{l}\text { Comment: insufficient detail provided of sequential numbering or whether } \\
\text { envelopes were opaque }\end{array}$ \\
\hline $\begin{array}{l}\text { Blinding of participants } \\
\text { and personnel (perfor- } \\
\text { mance bias) } \\
\text { All outcomes }\end{array}$ & Low risk & $\begin{array}{l}\text { Quote from publication: "Supplement prescription was done by researchers } \\
\text { who knew the randomisation codes, and were not involved in outcome data } \\
\text { collection, nor data entry to allow blinding" }\end{array}$ \\
\hline
\end{tabular}


Potter 2001 (Continued)

Blinding of outcome as- Unclear risk Quote from publication: "The researchers who performed the anthropometry sessment (detection bias) and assessed the clinical outcomes, were blinded to the intervention status of All outcomes the patients"

\begin{tabular}{lll}
$\begin{array}{l}\text { Incomplete outcome data } \\
\text { (attrition bias) } \\
\text { All outcomes }\end{array}$ & Unclear risk & $\begin{array}{l}\text { Comment: outcomes reported in relation to BMI and TSF, but not BMI and TSF } \\
\text { data alone }\end{array}$ \\
\hline $\begin{array}{l}\text { Selective reporting (re- } \\
\text { porting bias) }\end{array}$ & High risk & $\begin{array}{l}\text { Comment: one or more outcomes of interest to the review were reported in- } \\
\text { completely, so they could not be entered into the meta-analysis }\end{array}$
\end{tabular}

Other bias High risk Comment: in the well-nourished group, only $1 / 2$ were sequentially ran-

\begin{tabular}{|c|c|}
\hline Methods & $\begin{array}{l}\text { Parallel randomised controlled clinical trial } \\
\text { Randomisation ratio: } 1.1 \\
\text { Superiority design }\end{array}$ \\
\hline Participants & $\begin{array}{l}\text { Inclusion criteria: older than } 65 \text { years, on a soft or normal diet } \\
\text { Exclusion criteria: medically unstable, active malignancy or HIV, creatinine }>260 \text { micromols/L, com- } \\
\text { plex dietary needs } \\
\text { Diagnostic criteria: nursing home residents }\end{array}$ \\
\hline Interventions & $\begin{array}{l}\text { Number of trial centres: } 1 \\
\text { Treatment before trial: none specific }\end{array}$ \\
\hline Outcomes & Outcomes reported in abstract of publication: no abstract \\
\hline Study details & $\begin{array}{l}\text { Run-in period: none } \\
\text { Was trial terminated early: no }\end{array}$ \\
\hline Publication details & $\begin{array}{l}\text { Language of publication: English } \\
\text { Funding: non-commercial funding - Johns Hopkins University Fund for Geriatric Medicine and Nursing } \\
\text { Publication status: peer review journal }\end{array}$ \\
\hline Stated aim for study & $\begin{array}{l}\text { Quote from publication: "To determine the feasibility of implementing a comprehensive buffet-style } \\
\text { dining program and to determine the impact of the program on weight and biochemical indicators of } \\
\text { nutritional status among nursing home residents..." }\end{array}$ \\
\hline Notes & - \\
\hline
\end{tabular}

\section{Risk of bias}

\begin{tabular}{lll}
\hline Bias & Authors' judgement & Support for judgement \\
\hline $\begin{array}{l}\text { Random sequence genera- } \\
\text { tion (selection bias) }\end{array}$ & Unclear risk & Quote from paper: described as "subjects were randomised to participate"
\end{tabular}


Remsburg 2001 (Continued)

Comment: no details of procedure provided

\begin{tabular}{lll}
\hline $\begin{array}{l}\text { Allocation concealment } \\
\text { (selection bias) }\end{array}$ & Unclear risk & Comment: no detail \\
\hline $\begin{array}{l}\text { Blinding of participants } \\
\text { and personnel (perfor- } \\
\text { mance bias) } \\
\text { All outcomes }\end{array}$ & Unclear risk & Comment: no information \\
\hline $\begin{array}{l}\text { Blinding of outcome as- } \\
\text { sessment (detection bias) } \\
\text { All outcomes }\end{array}$ & Unclear risk & Comment: no information \\
\hline $\begin{array}{l}\text { Incomplete outcome data } \\
\text { (attrition bias) } \\
\text { All outcomes }\end{array}$ & Low risk & Comment: reported in footnotes of table 2 \\
\hline $\begin{array}{l}\text { Selective reporting (re- } \\
\text { porting bias) }\end{array}$ & Unclear risk & Comment: insufficient information to judge \\
\hline $\begin{array}{l}\text { Other bias } \\
\text { L }\end{array}$ & Low risk & $\begin{array}{l}\text { Comment: baseline characteristics comparable. Significantly more men in the } \\
\text { usual care }\end{array}$
\end{tabular}

\section{Salva 2011}

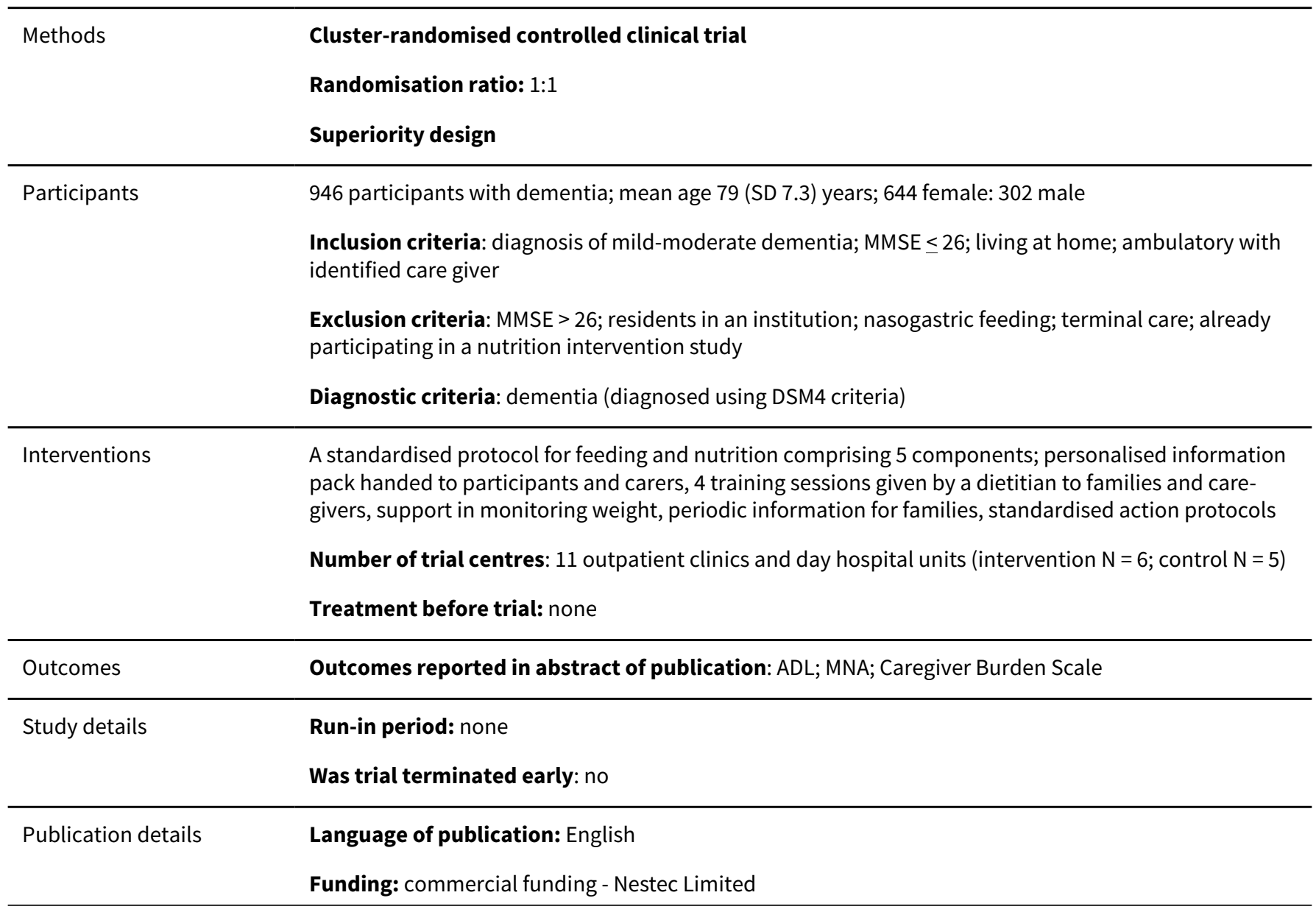


Salva 2011 (Continued)

Publication status: peer review journal

Stated aim for study

Quote from publication: "To assess the effectiveness of a health and nutrition programme (NurtiALZ) versus usual care on functional level in elderly people with dementia living at home, as well as on clinical practice related to nutrition and on the caregivers burden"

Notes

\section{Risk of bias}

\begin{tabular}{lll}
\hline Bias & Authors' judgement & Support for judgement \\
\hline $\begin{array}{l}\text { Random sequence genera- } \\
\text { tion (selection bias) }\end{array}$ & Unclear risk & $\begin{array}{l}\text { Quote from paper: "The unit of randomisation was the medical centres..." } \\
\text { Comment: insufficient detail of the method provided }\end{array}$ \\
\hline $\begin{array}{l}\text { Allocation concealment } \\
\text { (selection bias) }\end{array}$ & Unclear risk & Comment: not described \\
\hline $\begin{array}{l}\text { Blinding of participants } \\
\text { and personnel (perfor- } \\
\text { mance bias) } \\
\text { All outcomes }\end{array}$ & Unclear risk & Comment: not described \\
\hline
\end{tabular}

Blinding of outcome as- Unclear risk Comment: not described

sessment (detection bias)

All outcomes

Incomplete outcome data Low risk Comment: all participants and dropouts fully accounted for
(attrition bias)

All outcomes

Selective reporting (re- Low risk Comment: all fully reported
porting bias)

Other bias High risk Assessment of risk of bias in cluster-randomised trials

(1) Recruitment bias: no

(2) Baseline imbalance: frail status

(3) Loss of clusters: no

(4) Incorrect analysis: no

(5) Comparability with individually randomised trials/different types of clusters: different types of clusters

\begin{tabular}{ll}
\hline Methods & Cross-over randomised controlled clinical trial \\
Randomisation ratio: $1: 1$ \\
Superiority design
\end{tabular}


Exclusion criteria: chewing or swallowing dysfunction, need for feeding assistance, an eating disorder, depression, impaired functional status, dementia, $\mathrm{BMI} \leq 30 \mathrm{~kg} / \mathrm{m}^{2}$, medically-restricted diet on oral nutritional supplements, on orexigenic aids, regularly skip meals, smoke, more than 1 alcoholic drink per day

Diagnostic criteria: not stated

Interventions Number of trial centres: not applicable, participants are free-living

Treatment before trial: not stated

$\begin{array}{ll}\text { Outcomes } & \begin{array}{l}\text { Outcomes reported in abstract of publication: energy intake, key macro-nutrients and micronutri- } \\ \text { ents are mentioned but data not presented }\end{array}\end{array}$
ents are mentioned but data not presented

\begin{tabular}{ll}
\hline Study details & $\begin{array}{l}\text { Run-in period: none } \\
\text { Was trial terminated early: no }\end{array}$ \\
\hline Publication details & Language of publication: English \\
& Funding: non-commercial funding - Retirement research Foundation, Chicago, Illinois \\
& Publication status: peer review journal \\
\hline Stated aim for study & $\begin{array}{l}\text { Quote from publication: "To determine whether enhancing the energy density of food items regularly } \\
\text { served in a home delivered meals programme would increase lunch and 24 hour energy and nutrient } \\
\text { intakes" }\end{array}$
\end{tabular}

\section{Notes}

\section{Risk of bias}

\begin{tabular}{lll}
\hline Bias & Authors' judgement & Support for judgement \\
\hline $\begin{array}{l}\text { Random sequence genera- } \\
\text { tion (selection bias) }\end{array}$ & Unclear risk & $\begin{array}{l}\text { Quote from paper: "The experiment used a randomized crossover within-sub- } \\
\text { jects design" } \\
\text { Comment: insufficient detail of method provided }\end{array}$ \\
\hline $\begin{array}{l}\text { Allocation concealment } \\
\text { (selection bias) }\end{array}$ & Unclear risk & Comment: no detail \\
\hline $\begin{array}{l}\text { Blinding of participants } \\
\begin{array}{l}\text { and personnel (perfor- } \\
\text { mance bias) } \\
\text { All outcomes }\end{array}\end{array}$ & Unclear risk & Comment: no information \\
\hline
\end{tabular}

\begin{tabular}{|c|c|c|}
\hline $\begin{array}{l}\text { Blinding of outcome as- } \\
\text { sessment (detection bias) } \\
\text { All outcomes }\end{array}$ & Unclear risk & Comment: no information \\
\hline $\begin{array}{l}\text { Incomplete outcome data } \\
\text { (attrition bias) } \\
\text { All outcomes }\end{array}$ & Unclear risk & $\begin{array}{l}\text { Comment: } 7 \text { participants dropped out but reasons not given, and unclear } \\
\text { from which group they dropped out }\end{array}$ \\
\hline $\begin{array}{l}\text { Selective reporting (re- } \\
\text { porting bias) }\end{array}$ & Low risk & Comment: the outcomes specified in the methods are reported in the results \\
\hline
\end{tabular}


Silver 2008 (Continued)

Other bias Unclear risk Comment: no table of baseline characteristics. The information on need for assistance with shopping and preparation of food and recent weight loss suggests heterogeneity in the population

Simmons 2008

\begin{tabular}{|c|c|}
\hline Methods & $\begin{array}{l}\text { Cluster- and cross-over randomised controlled clinical trial } \\
\text { Randomisation ratio: } 1: 1 \\
\text { Superiority design }\end{array}$ \\
\hline Participants & $\begin{array}{l}\text { Inclusion criteria: long-stay residents in a care home; free of feeding tube, not receiving palliative care, } \\
\text { not on planned weight loss diet } \\
\text { Exclusion criteria: not explicitly stated } \\
\text { Diagnostic criteria: not stated }\end{array}$ \\
\hline Interventions & $\begin{array}{l}\text { Number of trial centres: } 4 \text { care homes } \\
\text { Treatment before trial: not stated }\end{array}$ \\
\hline Outcomes & $\begin{array}{l}\text { Outcomes reported in abstract of publication: energy intake, weight change; staff time to provide in- } \\
\text { terventions }\end{array}$ \\
\hline Study details & $\begin{array}{l}\text { At baseline all eligible participants were assessed for responsiveness (15\% increase in energy intake) to } \\
\text { one of } 2 \text { interventions (i.e. feeding assistance or between-meal snacks). This was a 2-phase cross-over } \\
\text { trial where residents not eligible in the first phase were re-evaluated for possible inclusion in the sec- } \\
\text { ond phase and residents included in the first phase were re-evaluated and could become ineligible for } \\
\text { the second phase (based on adequacy of energy intake) } \\
\text { Run-in period: not stated } \\
\text { Was trial terminated early: no }\end{array}$ \\
\hline
\end{tabular}

\section{Publication details Language of publication: English}

Funding: non-commercial funding - National Institute of Aging and National Institute of Health, University of California, LA

Publication status: peer review journal

Stated aim for study

Quote from publication: "To evaluate the effect of two feeding assistance interventions (meal time assistants and between meal snack delivery) on residents oral food and fluid intake, BMI and weight status when maintained by research staff for 24 weeks"

Notes

\section{Risk of bias}

\begin{tabular}{lll}
\hline Bias & Authors' judgement & Support for judgement \\
\hline $\begin{array}{l}\text { Random sequence genera- } \\
\text { tion (selection bias) }\end{array}$ & Low risk & $\begin{array}{l}\text { Quote from publication: "Participants were randomised at the facility level..., } \\
\text { the four nursing homes were identified as intervention or control (in pairs of } \\
\text { two) using a toss of the coin...." }\end{array}$ \\
\hline
\end{tabular}


Simmons 2008 (Continued)

Allocation concealment Unclear risk
(selection bias)

Blinding of participants

High risk

Comment: no blinding and outcome likely to be influenced by lack of blinding and personnel (performance bias)

All outcomes

\section{Blinding of outcome as-} sessment (detection bias)

High risk

Comment: no blinding and outcome likely to be influenced by lack of blinding

All outcomes

Incomplete outcome data Unclear risk

(attrition bias)

All outcomes
Comment: numbers are described in text and appendix. Mortality given as a reason for most dropouts (58\%), but the remaining reasons are not described.

Selective reporting (re- Unclear risk Comment: insufficient information to judge porting bias)

Other bias High risk

Comment: baseline characteristics presented for total numbers of participants in each group (phase 1 and 2 combined)

\section{Assessment of risk of bias in cluster-randomised trials}

(1) Recruitment bias: no

(2) Baseline imbalance: frail status

(3) Loss of clusters: no

(4) Incorrect analysis: no

(5) Comparability with individually randomised trials/different types of clusters: different types of clusters

Simmons 2010

$\begin{array}{ll}\text { Methods } & \text { Parallel randomised controlled clinical trial } \\ & \text { Randomisation ratio: } 1: 1: 1 \\ & \text { Superiority design }\end{array}$

Participants Inclusion criteria: long stay residents; free of feeding tube; not receiving hospice care; identified for nutritional supplementation

Exclusion criteria: not stated

Diagnostic criteria:

Interventions Number of trial centres: 3

Treatment before trial: not stated

\begin{tabular}{ll}
\hline Outcomes & Outcomes reported in abstract of publication: energy intake, staff time and costs \\
\hline Study details & Run-in period: not stated \\
& Was trial terminated early: no \\
\hline
\end{tabular}


Simmons 2010 (Continued)

Publication details Language of publication: English

Funding: non-commercial funding - National Alzheimer's Association and National Institute for Aging Publication status: peer review journal

Stated aim for study $\quad \begin{aligned} & \text { Quote from publication: "To determine the cost effectiveness of supplements relative to offering resi- } \\ & \text { dents snack foods and fluids between meals to increase caloric intake" }\end{aligned}$

\begin{tabular}{|c|c|c|}
\hline Notes & - & \\
\hline \multicolumn{3}{|l|}{ Risk of bias } \\
\hline Bias & Authors' judgement & Support for judgement \\
\hline $\begin{array}{l}\text { Random sequence genera- } \\
\text { tion (selection bias) }\end{array}$ & Unclear risk & $\begin{array}{l}\text { Quote from paper: "Participants.....were randomised into one of three groups" } \\
\text { Comment: insufficient detail of method provided }\end{array}$ \\
\hline $\begin{array}{l}\text { Allocation concealment } \\
\text { (selection bias) }\end{array}$ & Unclear risk & Comment: no detail provided \\
\hline $\begin{array}{l}\text { Blinding of participants } \\
\text { and personnel (perfor- } \\
\text { mance bias) } \\
\text { All outcomes }\end{array}$ & Unclear risk & Comment: insufficient detail \\
\hline
\end{tabular}

\begin{tabular}{|c|c|c|}
\hline $\begin{array}{l}\text { Blinding of outcome as- } \\
\text { sessment (detection bias) } \\
\text { All outcomes }\end{array}$ & Unclear risk & Comment: insufficient detail \\
\hline $\begin{array}{l}\text { Incomplete outcome data } \\
\text { (attrition bias) } \\
\text { All outcomes }\end{array}$ & Low risk & Comment: fully reported \\
\hline $\begin{array}{l}\text { Selective reporting (re- } \\
\text { porting bias) }\end{array}$ & Unclear risk & Comment: insufficient detail to judge \\
\hline Other bias & Unclear risk & $\begin{array}{l}\text { Comment: baseline characteristics reported for whole study population and } \\
\text { not according to group allocation }\end{array}$ \\
\hline
\end{tabular}

Smoliner 2008

\begin{tabular}{ll}
\hline Methods & Cluster-randomised controlled clinical trial \\
& Randomisation ratio: $1: 1$ \\
& Superiority design \\
\hline Participants & Inclusion criteria: MNA score $\leq 23.5$ points \\
& $\begin{array}{l}\text { Exclusion criteria: MNA }>23.5 \text { points, severe cognitive impairment, on enteral feeding, hospital stay } \geq 6 \\
\text { days during the study period }\end{array}$ \\
& Diagnostic criteria: not stated \\
\hline Interventions & Number of trial centres: 3 care homes
\end{tabular}

Number of trial centres: 3 care homes 
Smoliner 2008 (Continued)

Treatment before trial: not stated

Outcomes

Outcomes reported in abstract of publication: protein and energy intake, nutritional status and body composition, muscle function and physical function

$\begin{array}{ll}\text { Study details } & \text { Run-in period: not stated } \\ \text { Was trial terminated early: no }\end{array}$

Publication details

\section{Language of publication: English}

Funding: commercial funding - Schubert Holding Ag \& Co, KG

Publication status: peer review journal

\section{Stated aim for study}

Quote from publication: "To evaluate the effect of a 12 week nutritional intervention with protein and energy enriched food and snacks on nutritional and functional status in elderly nursing home residents at risk of malnutrition"

\section{Notes \\ Risk of bias}

\begin{tabular}{lll}
\hline Bias & Authors' judgement & Support for judgement \\
\hline $\begin{array}{l}\text { Random sequence genera- } \\
\text { tion (selection bias) }\end{array}$ & Unclear risk & Quote from paper: "Randomisation was done according to ward...." \\
& & Comment: insufficient detail of the method provided \\
\hline $\begin{array}{l}\text { Allocation concealment } \\
\text { (selection bias) }\end{array}$ & Unclear risk & Comment: no detail \\
\hline $\begin{array}{l}\text { Blinding of participants } \\
\text { and personnel (perfor- } \\
\text { mance bias) } \\
\begin{array}{l}\text { All outcomes } \\
\text { Ond }\end{array}\end{array}$ & Unclear risk & Comment: no information \\
\hline
\end{tabular}

\begin{tabular}{ll}
\hline Blinding of outcome as- & Unclear risk \\
sessment (detection bias) & \\
All outcomes & \\
\hline
\end{tabular}

Incomplete outcome data Low risk
(attrition bias)

All outcomes

\begin{tabular}{|c|c|c|}
\hline $\begin{array}{l}\text { Selective reporting (re- } \\
\text { porting bias) }\end{array}$ & Unclear risk & Comment: no protocol available \\
\hline Other bias & High risk & $\begin{array}{l}\text { Assessment of risk of bias in cluster-randomised trials } \\
\text { (1) Recruitment bias: unclear } \\
\text { (2) Baseline imbalance: length of stay, number of medications, SF-36 physical } \\
\text { functioning score } \\
\text { (3) Loss of clusters: unclear } \\
\text { (4) incorrect analysis: yes } \\
\text { (5) Comparability with individually randomised trials/different types of clus- } \\
\text { ters: unclear }\end{array}$ \\
\hline
\end{tabular}


Splett 2003

\begin{tabular}{ll} 
Methods & Cluster-randomised controlled clinical trial \\
& Randomisation ratio: $1: 1$ \\
& Superiority design \\
\hline Participants & Inclusion criteria: people entering residential care facilities with service provided by a dietitian \\
& $\begin{array}{l}\text { Exclusion criteria: people entering a hospice or respite care programme or those expected to have a } \\
\text { stay< } 30 \text { days } \\
\text { Diagnostic criteria: varied }\end{array}$ \\
\hline
\end{tabular}

\begin{tabular}{ll}
\hline Interventions & Number of trial centres: 29 \\
& Treatment before trial: $57 \%$ intervention group and $61 \%$ usual care had previous dietary modification \\
and $25 \%$ intervention and $35 \%$ control received help at mealtimes
\end{tabular}

Outcomes Outcomes reported in abstract of publication: rate of unintentional weight loss, weight status 90 days after admission and weight status 90 days after identification of unintentional weight loss

\begin{tabular}{|c|c|c|}
\hline Study details & \multicolumn{2}{|c|}{$\begin{array}{l}\text { Run-in period: none } \\
\text { Was trial terminated early: no }\end{array}$} \\
\hline \multirow[t]{3}{*}{ Publication details } & \multirow{3}{*}{\multicolumn{2}{|c|}{$\begin{array}{l}\text { Language of publication: English } \\
\text { Funding: not stated } \\
\text { Publication status: peer review journal }\end{array}$}} \\
\hline & & \\
\hline & & \\
\hline Stated aim for study & \multicolumn{2}{|c|}{$\begin{array}{l}\text { Quote from publication: "To assess the effectiveness of a new medical nutrition therapy protocol for } \\
\text { the prevention and treatment of unintentional weight loss and describe nutrition assessment and in- } \\
\text { tervention activities of dietitians" }\end{array}$} \\
\hline Notes & - & \\
\hline \multicolumn{3}{|l|}{ Risk of bias } \\
\hline Bias & Authors' judgement & Support for judgement \\
\hline $\begin{array}{l}\text { Random sequence genera- } \\
\text { tion (selection bias) }\end{array}$ & Low risk & $\begin{array}{l}\text { Quote from publication: ".. facilities were randomly assigned to either the } \\
\text { medical nutrition therapy protocol care group (MNTPC) or the usual care (UC) } \\
\text { group using a random numbers table" }\end{array}$ \\
\hline $\begin{array}{l}\text { Allocation concealment } \\
\text { (selection bias) }\end{array}$ & Unclear risk & Comment: not described \\
\hline $\begin{array}{l}\text { Blinding of participants } \\
\text { and personnel (perfor- } \\
\text { mance bias) } \\
\text { All outcomes }\end{array}$ & Unclear risk & Comment: not described \\
\hline $\begin{array}{l}\text { Blinding of outcome as- } \\
\text { sessment (detection bias) } \\
\text { All outcomes }\end{array}$ & Unclear risk & Comment: not described \\
\hline
\end{tabular}


Splett 2003 (Continued)

Incomplete outcome data Low risk Comment: fully described
(attrition bias)

All outcomes

\begin{tabular}{|c|c|c|}
\hline $\begin{array}{l}\text { Selective reporting (re- } \\
\text { porting bias) }\end{array}$ & Low risk & Comment: all outcomes reported \\
\hline Other bias & High risk & $\begin{array}{l}\text { Assessment of risk of bias in cluster-randomised trials } \\
\text { (1) Recruitment bias: unclear } \\
\text { (2) Baseline imbalance: number of diagnoses } \\
\text { (3) Loss of clusters: unclear } \\
\text { (4) Incorrect analysis: yes } \\
\text { (5) Comparability with individually randomised trials/different types of clus- } \\
\text { ters: unclear }\end{array}$ \\
\hline
\end{tabular}

Taylor 2006

$\begin{array}{ll}\text { Methods } & \text { Cross-over randomised controlled clinical trial } \\ \text { Randomisation ratio: } 1: 1 \\ \text { Superiority design }\end{array}$

$\begin{array}{ll}\text { Participants } & \begin{array}{l}\text { Inclusion criteria: aged } \geq 65 \text { years; dysphagia (diagnosed by swallowing team); receiving a texture } \\ \text { modified diet }\end{array}\end{array}$

Exclusion criteria: tube-fed; medically unstable; receiving a diabetic diet

Diagnostic criteria: not stated

\begin{tabular}{ll}
\hline Interventions & $\begin{array}{l}\text { Number of trial centres: } 1 \\
\text { Treatment before trial: not stated }\end{array}$ \\
\hline Outcomes & Outcomes reported in abstract of publication: energy and fluid intakes \\
\hline Study details & Run-in period: not stated \\
& Was trial terminated early: no \\
\hline Publication details & Language of publication: English \\
& $\begin{array}{l}\text { Funding: } \text { non-commercial funding - Canadian Foundation for Dietetic Research } \\
\text { Publication status: peer review journal }\end{array}$
\end{tabular}
Stated aim for study
Quote from publication: "To determine whether serving a 5 meal pattern versus a traditional 3 meal pattern would improve energy intake among elderly, extended care residents with dysphagia"

\begin{tabular}{ll}
\hline Notes & - \\
\hline Risk of bias & Authors' judgement Support for judgement \\
\hline Bias & Suld \\
\hline
\end{tabular}


Taylor 2006 (Continued)
Random sequence genera- Unclear risk tion (selection bias)
Quote from paper: "Participants were randomly assigned to one of two groups."

Comment: insufficient detail of method provided

\begin{tabular}{lll}
\hline $\begin{array}{l}\text { Allocation concealment } \\
\text { (selection bias) }\end{array}$ & Unclear risk & Comment: not reported \\
\hline $\begin{array}{l}\text { Blinding of participants } \\
\text { and personnel (perfor- } \\
\text { mance bias) }\end{array}$ & Unclear risk & Comment: not reported \\
All outcomes & \\
\hline
\end{tabular}

$\begin{array}{lll}\text { Blinding of outcome as- } & \text { Unclear risk } & \text { Comment: not reported } \\ \text { sessment (detection bias) } & \end{array}$

All outcomes

\begin{tabular}{lll}
\hline $\begin{array}{l}\text { Incomplete outcome data } \\
\text { (attrition bias) } \\
\text { All outcomes }\end{array}$ & Unclear risk & $\begin{array}{l}\text { Comment: reason for dropouts reported, however unclear from which groups } \\
\text { they dropped out }\end{array}$ \\
\hline $\begin{array}{l}\text { Selective reporting (re- } \\
\text { porting bias) }\end{array}$ & Unclear risk & Comment: insufficient information to judge \\
\hline Other bias & Unclear risk & $\begin{array}{l}\text { Comment: baseline characteristics reported in the text; homogeneous popu- } \\
\text { lation }\end{array}$ \\
\hline
\end{tabular}

Van den Berg 2015

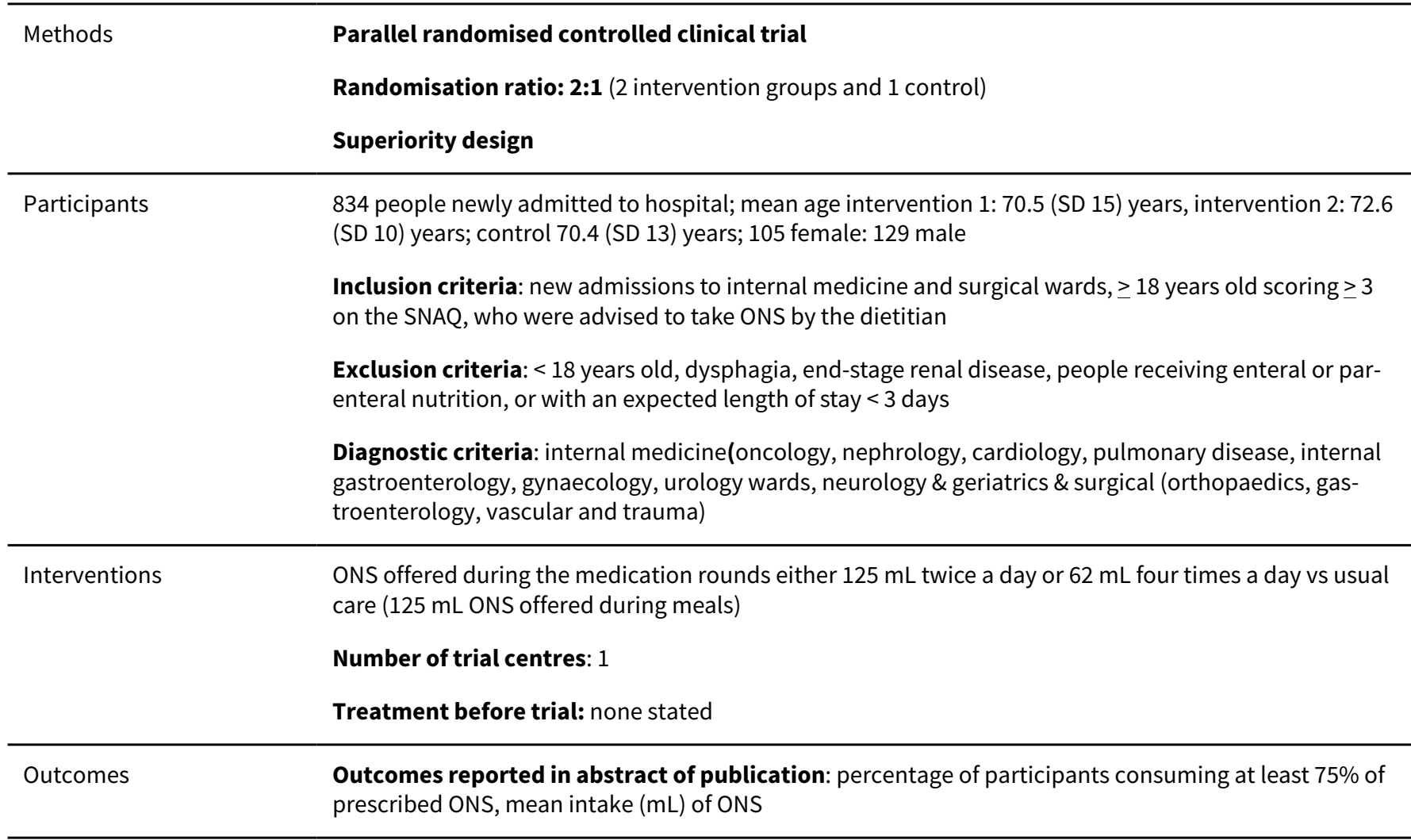


Van den Berg 2015 (Continued)
Study details
Run-in period: none
Was trial terminated early: no

Publication details Language of publication: English

Funding: Study funded by Deventer Hospital, the Netherlands. (No commercial funding and the ONS was not donated)

Publication status: peer review journal

Stated aim for study

Quote "to investigate whether the distribution of ONS during medication rounds, either in 2 higher volumes or in 4 lower volumes, would increase the intake of the supplements and to evaluate its effects on patient compliance with consumption of the ONS

\begin{tabular}{|c|c|c|}
\hline Notes & - & \\
\hline \multicolumn{3}{|l|}{ Risk of bias } \\
\hline Bias & Authors' judgement & Support for judgement \\
\hline $\begin{array}{l}\text { Random sequence genera- } \\
\text { tion (selection bias) }\end{array}$ & Low risk & Quote from publication: "Computerised random number system" \\
\hline $\begin{array}{l}\text { Allocation concealment } \\
\text { (selection bias) }\end{array}$ & Low risk & Quote from publication: "concealed blinded envelopes" \\
\hline $\begin{array}{l}\text { Blinding of participants } \\
\text { and personnel (perfor- } \\
\text { mance bias) } \\
\text { All outcomes }\end{array}$ & High risk & $\begin{array}{l}\text { Quote from publication: "it is not possible to perform a blinded study for nu- } \\
\text { tritional support" } \\
\text { Comment: the participants and personnel were not blinded to intervention } \\
\text { group }\end{array}$ \\
\hline $\begin{array}{l}\text { Blinding of outcome as- } \\
\text { sessment (detection bias) } \\
\text { All outcomes }\end{array}$ & High risk & Comment: outcomes were not assessed blinded to study group \\
\hline $\begin{array}{l}\text { Incomplete outcome data } \\
\text { (attrition bias) } \\
\text { All outcomes }\end{array}$ & Low risk & $\begin{array}{l}\text { Comment: attrition fully described, } 31 \text { patients refused the ONS during the } \\
\text { study but were included in the analysis, } 42 \text { patients were discharged within } 2 \\
\text { days of follow-up and were excluded from analyses }\end{array}$ \\
\hline $\begin{array}{l}\text { Selective reporting (re- } \\
\text { porting bias) }\end{array}$ & Low risk & Comment: all specified outcomes were reported \\
\hline Other bias & Low risk & $\begin{array}{l}\text { Comment: baseline characteristics fully reported and groups similar at base- } \\
\text { line }\end{array}$ \\
\hline
\end{tabular}

Van Ort 1995

\begin{tabular}{ll}
\hline Methods & Parallel randomised controlled clinical trial \\
& Randomisation ratio: $1: 1$ \\
& Superiority design \\
\hline Participants & $\begin{array}{l}\text { Inclusion criteria: required feeding assistance by a caregiver (nurse and/or nursing assistant), were } \\
\text { able to sit in a chair for feeding, were responsive to human interaction, were not usually restrained dur- } \\
\text { ing feeding, were not usually combative }\end{array}$ \\
\hline
\end{tabular}


Van Ort 1995 (Continued)

Exclusion criteria: not given

Diagnostic criteria: not stated

Interventions
$\begin{aligned} & \text { vention). It was unclear whether interventions were given together, or given one after the other. } 2 \text { com- } \\ & \text { plete lunches and two dinners in week 1, and } 3 \text { lunches and dinners in week } 2 \text { were video tape-record- } \\ & \text { ed }\end{aligned}$

Number of trial centres: 1

Treatment before trial: none

\begin{tabular}{ll}
\hline Outcomes & Outcomes reported in abstract of publication: no abstract \\
\hline Study details & Run-in period: no \\
& Was trial terminated early: no \\
\hline Publication details & Language of publication: English \\
& $\begin{array}{l}\text { Funding: non-commercial funding; "This study was supported by a } 1991 \text { Christian P. Voltz Memorial Pi- } \\
\text { lot Grant Award from the Alzheimers Association" } \\
\text { Publication status: peer review journal }\end{array}$ \\
\hline Stated aim for study & $\begin{array}{l}\text { Quote from publication: "the interventions were designed to first create a feeding context or environ- } \\
\text { ment that promoted function by being as "near normal" as possible and by removing barriers to func- } \\
\text { tion, and second to provide randomly selected patients with behavioural prompts, cues and reinforce- } \\
\text { ments for self feeding approximations" }\end{array}$ \\
\hline
\end{tabular}

Notes

\section{Risk of bias}

Bias Authors' judgement Support for judgement

Random sequence genera- Unclear risk tion (selection bias)

Quote from paper: "Then four of the eight subjects were randomly selected to receive the intervention...."

Comment: insufficient details of the procedure

\begin{tabular}{lll}
\hline $\begin{array}{l}\text { Allocation concealment } \\
\text { (selection bias) }\end{array}$ & Unclear risk & Comment: insufficient information to permit judgement \\
\hline $\begin{array}{l}\text { Blinding of participants } \\
\text { and personnel (perfor- } \\
\text { mance bias) } \\
\text { All outcomes }\end{array}$ & Unclear risk & $\begin{array}{l}\text { Comment: insufficient information to permit judgement - study stated "the } \\
\text { project research associates were blind to the specific study hypothesis", how- } \\
\text { ever their role in the study unclear }\end{array}$ \\
\hline $\begin{array}{l}\text { Blinding of outcome as- } \\
\text { sessment (detection bias) }\end{array}$ & Unclear risk & $\begin{array}{l}\text { Comment: the research associates were responsible for implementing the be- } \\
\text { havioural intervention. } \text { On analysing the video tapes, they were blinded to the } \\
\text { study hypotheses, however no statement to say they were blinded to the study } \\
\text { interventions }\end{array}$ \\
\hline $\begin{array}{l}\text { Incomplete outcome data } \\
\text { (attrition bias) } \\
\text { All outcomes }\end{array}$ & Unclear risk & $\begin{array}{l}\text { Comment: insufficient reporting of attrition/exclusions to permit judgement. } \\
\text { The number of dropouts were stated, however it was unclear from which } \\
\text { group }\end{array}$ \\
\hline
\end{tabular}


Van Ort 1995 (Continued)

Selective reporting (reporting bias)

High risk

Comment: the study report failed to include results for key outcomes that would be expected to have been reported for such a study - the study does not provide any data

Other bias Unclear risk Comment: no baseline characteristics reported, therefore insufficient information to assess whether an important risk of bias exists

ADL: activities of daily living; BMI: body mass index; EdFED: Edinburgh Feeding Evaluation in Dementia; HIV: human immunodeficiency virus; MAC: mid-arm circumference; MAMC: mid-arm muscle circumference; MMSE: Mini Mental State Examination; MNA: Mini Nutritional Assessment; MoW: meals on wheels; MSG: monosodium glutamate; MUAC: mid upper-arm circumference; NRS: Nutritional Risk Screening; ONS: oral nutritional supplement; PGCMS: Philadelphia Geriatric Centre Morale Scale; SD: standard deviation; SE: standard error; SGA: subjective global assessment; SIP: sickness impact profile; SNAQ: Simplified Nutritional Appetite Questionnaire; TSF: triceps skin fold

\section{Characteristics of excluded studies [ordered by study ID]}

\begin{tabular}{ll}
\hline Study & Reason for exclusion \\
\hline Aleman-Mateo 2012 & $\begin{array}{l}\text { Not a supportive intervention in nutritional care; intervention included individual advice on taking } \\
\text { ONS as participants were free-living }\end{array}$ \\
\hline
\end{tabular}

\begin{tabular}{ll}
\hline Allman 1990 & $\begin{array}{l}\text { Not a supportive intervention in nutritional care; ONS prescribed on an individualised basis, as di- } \\
\text { etary advice was given, and participants had to follow instructions to take ONS at home }\end{array}$
\end{tabular}

\begin{tabular}{ll}
\hline Arias 2008 & $\begin{array}{l}\text { Not a supportive intervention; intervention is an ONS with no mention of supportive strategy to } \\
\text { support administration }\end{array}$
\end{tabular}

\begin{tabular}{ll}
\hline Asplund 2000 & $\begin{array}{l}\text { Not a supportive intervention in nutritional care; looked at the effect of residence in an acute geri- } \\
\text { atrics-based ward, outcomes not relevant to this review }\end{array}$ \\
\hline Baldwin 2011 & $\begin{array}{l}\text { Not a supportive intervention in nutritional care; individualised interventions therefore partici- } \\
\text { pants were required to understand and follow instructions }\end{array}$ \\
\hline Banerjee 1978 & $\begin{array}{l}\text { Not a supportive intervention in nutritional care; intervention was described, however no clear or- } \\
\text { ganisational component to the intervention was given }\end{array}$
\end{tabular}

Bauer $2005 \quad \begin{aligned} & \text { Not a supportive intervention in nutritional care; intervention was described, however no clear } \\ & \text { organisational component to the intervention was given. The intervention was also micronutri- } \\ & \text { ent-specific }\end{aligned}$

Beattie $2000 \quad$ Not a supportive intervention in nutritional care; no clear organisational component to the intervention was described, and the intervention was continued post hospital discharge, therefore participants would have been given individual advice on taking ONS

\begin{tabular}{ll}
\hline Beck 2008 & $\begin{array}{l}\text { Not a supportive intervention in nutritional care; but a multicomponent intervention, therefore un- } \\
\text { able to extract specific effect of nutrition component }\end{array}$ \\
\hline Benati 2001 & $\begin{array}{l}\text { The intervention included supplementation with oNS but there was no indication that a supportive } \\
\text { protocol was used to support the intervention }\end{array}$ \\
\hline Bonjour 2011 & $\begin{array}{l}\text { Not a supportive intervention in nutritional care; intervention involved calcium and vitamin D sup- } \\
\text { plementation }\end{array}$ \\
\hline Bonjour 2012 & Not a supportive intervention in nutritional care; unclear nutritional risk of participants \\
\hline Bonnefoy 2003 & $\begin{array}{l}\text { Not a supportive intervention in nutritional care but a multicomponent intervention, therefore un- } \\
\text { able to extract specific effect of nutrition component }\end{array}$
\end{tabular}

Supportive interventions for enhancing dietary intake in malnourished or nutritionally at-risk adults (Review) 


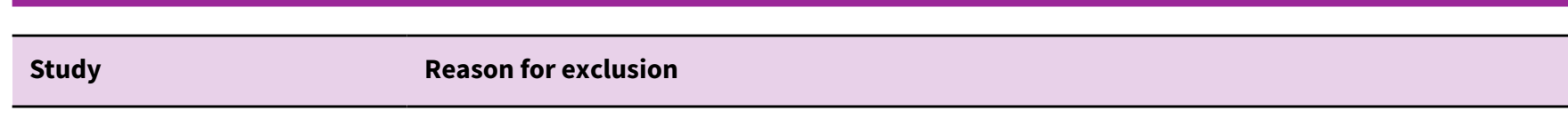

\begin{tabular}{ll}
\hline Bos 2001 & Not a RCT \\
\hline Botella-Carretero 2008 & $\begin{array}{l}\text { Not a supportive intervention in nutritional care; intervention continued post hospital discharge, } \\
\text { therefore participants would have been given individual advice on taking ONS }\end{array}$ \\
\hline Botella-Carretero 2010 & $\begin{array}{l}\text { Not a supportive intervention in nutritional care; ONS prescribed on an individualised basis, and } \\
\text { tailored to texture and estimated nutritional requirements }\end{array}$ \\
\hline
\end{tabular}

Boudville 2003 Not a supportive intervention in nutritional care; intervention given to outpatients, therefore par-
ticipants would have been given individual advice on taking ONS

\begin{tabular}{ll}
\hline Bunout 1989 & Not a supportive intervention in nutritional care; ONS tailored to body weight/nutritional require- \\
ments, therefore prescribed on an individualised basis
\end{tabular}

\begin{tabular}{ll}
\hline Bunout 2001 & $\begin{array}{l}\text { Not a randomised control trial; the nutritional intervention was not randomised but the exercise in- } \\
\text { tervention was }\end{array}$
\end{tabular}

\begin{tabular}{ll}
\hline Carlsson 2011 & $\begin{array}{l}\text { Not a supportive intervention in nutritional care but a multicomponent intervention, therefore un- } \\
\text { able to extract specific effect of nutrition component }\end{array}$ \\
\hline Carnaby 2006 & $\begin{array}{l}\text { Not a supportive intervention in nutritional care; intervention specific to stroke participants with } \\
\text { dysphagia hence scope not considered broad enough to be a supportive intervention in nutritional } \\
\text { care }\end{array}$ \\
\hline
\end{tabular}

Charlin 2002 Not a supportive intervention in nutritional care; intervention given to outpatients, therefore par-
ticipants would have been given individual advice on taking ONS

\begin{tabular}{ll}
\hline Charras 2010 & Not a randomised controlled trial \\
\hline Chernoff 1990 & $\begin{array}{l}\text { Not a supportive intervention in nutritional care; artificial support was given via non oral route, en- } \\
\text { teral tube feeding }\end{array}$
\end{tabular}

\begin{tabular}{ll}
\hline Chin 2001 & Not a supportive intervention in nutritional care; micronutrient supplementation study; usual care \\
had non-enriched 'product'
\end{tabular}

Collins 2005 Not a supportive intervention in nutritional care; intervention given to outpatients, therefore par-
ticipants would have been given individual advice on taking ONS

Dangour 2011 Not a supportive intervention in nutritional care; intervention given to outpatients, therefore par-
ticipants would have been given individual advice on taking an ONS

\begin{tabular}{ll}
\hline De Jong 1999 & Not a supportive intervention in nutritional care; a micronutrient enrichment intervention \\
\hline de Sousa 2012 & $\begin{array}{l}\text { Not a supportive intervention in nutritional care; intervention was described, however no clear or- } \\
\text { ganisational component to the intervention was given }\end{array}$ \\
\hline Delmi 1990 & $\begin{array}{l}\text { Not a supportive intervention in nutritional care; intervention was described, however no clear or- } \\
\text { ganisational component to the intervention was given }\end{array}$ \\
\hline Dhanraj 1997 & $\begin{array}{l}\text { Not a supportive intervention in nutritional care; artificial support was given via non oral route (na- } \\
\text { sogastric feeding); no usual care comparison; some participants < 18 yrs; individualised nutritional } \\
\text { care given }\end{array}$ \\
\hline Dillabough 2011 & Not a RCT; article describing a pilot quality improvement project \\
\hline
\end{tabular}




\begin{tabular}{ll}
\hline Study & Reason for exclusion \\
\hline Edington 2004 & $\begin{array}{l}\text { Not a supportive intervention in nutritional care; ONS tailored to individual estimated nutritional } \\
\text { requirements, therefore prescribed on an individualised basis }\end{array}$ \\
\hline
\end{tabular}

$\begin{array}{ll}\text { Elkort } 1981 & \begin{array}{l}\text { Not a supportive intervention in nutritional care; ONS tailored to individual estimated nutritional } \\ \text { requirements, therefore prescribed on an individualised basis }\end{array}\end{array}$

\begin{tabular}{ll}
\hline Endevelt 2011 & Not a supportive intervention in nutritional care; intervention was individualised \\
\hline Eneroth 2004 & $\begin{array}{l}\text { Not a supportive intervention in nutritional care; intervention given to outpatients, therefore par- } \\
\text { ticipants would have been given individual advice on taking ONS }\end{array}$ \\
\hline Espaulella 2000 & $\begin{array}{l}\text { Not a supportive intervention in nutritional care; intervention was described, however no clear or- } \\
\text { ganisational component to the intervention was given }\end{array}$
\end{tabular}

$\begin{array}{ll}\text { Fiatarone } 1994 & \text { Not a supportive intervention in nutritional care but a multicomponent intervention, therefore un- } \\ \text { able to extract specific effect of nutrition component }\end{array}$

\begin{tabular}{ll}
\hline Forster 2005 & $\begin{array}{l}\text { Not a supportive intervention in nutritional care; intervention was described, however no clear or- } \\
\text { ganisational component to the intervention was given }\end{array}$
\end{tabular}

\begin{tabular}{ll}
\hline Gall 1998 & Not a RCT; controlled trial \\
\hline Gariballa 1998 & $\begin{array}{l}\text { Not a supportive intervention in nutritional care; intervention was described, however no clear or- } \\
\text { ganisational component to the intervention was given }\end{array}$
\end{tabular}

\begin{tabular}{ll}
\hline Gazzotti 2003 & $\begin{array}{l}\text { Not a supportive intervention in nutritional care; intervention continued post hospital discharge, } \\
\text { therefore participants would have been given individual advice on taking ONS. }\end{array}$ \\
\hline Gegerle 1986 & Not a RCT; a dietary survey \\
\hline Gil Gregorio 2003 & $\begin{array}{l}\text { Not a supportive intervention in nutritional care; intervention was described, however no clear or- } \\
\text { ganisational component to the intervention was given; unclear what the usual care received }\end{array}$
\end{tabular}

Goris $2003 \quad$ Not a supportive intervention in nutritional care; intervention continued post hospital discharge,
therefore participants would have been given individual advice on taking ONS.

\begin{tabular}{ll}
\hline Hogarth 1996 & $\begin{array}{l}\text { Not a supportive intervention in nutritional care; intervention was described, however no clear or- } \\
\text { ganisational component to the intervention was given }\end{array}$ \\
\hline Hopkinson 2010 & $\begin{array}{l}\text { Not a supportive intervention in nutritional care; study not aimed at increasing intake as related to } \\
\text { psychological/coping mechanisms }\end{array}$ \\
\hline Houles 2010 & $\begin{array}{l}\text { Not a supportive intervention in nutritional care but a multicomponent intervention, therefore un- } \\
\text { able to extract specific effect of nutrition component }\end{array}$ \\
\hline Hubbard 2008 & $\begin{array}{l}\text { Not a supportive intervention in nutritional care; intervention was on dietary advice vs ONS, so no } \\
\text { usual care comparison was given }\end{array}$ \\
\hline Hubsch 1992 & $\begin{array}{l}\text { Not a supportive intervention in nutritional care; intervention was described, however no clear or- } \\
\text { ganisational component to the intervention was given }\end{array}$ \\
\hline Huisman 2012 & Not a supportive intervention in nutritional care; dietary counselling intervention \\
\hline Isenring 2003 & Not a supportive intervention in nutritional care; dietary counselling intervention \\
\hline Isenring 2004 & Not a supportive intervention in nutritional care; dietary counselling intervention
\end{tabular}




\begin{tabular}{ll}
\hline Study & Reason for exclusion \\
\hline Jahnavi 2010 & Not a supportive intervention in nutritional care; individualised intervention \\
\hline James 2006 & $\begin{array}{l}\text { Not a supportive intervention in nutritional care; participants consumed ONS at will, intervention } \\
\text { not identical for all participants }\end{array}$ \\
\hline Johnson 1993 & Not a RCT; retrospective case control study \\
\hline Keele 1997 & $\begin{array}{l}\text { Not a supportive intervention in nutritional care; intervention continued post hospital discharge, } \\
\text { therefore participants would have been given individual advice on taking ONS }\end{array}$ \\
\hline Kikutani 2006 & $\begin{array}{l}\text { Not a supportive intervention in nutritional care; no usual care comparison was described; ONS in- } \\
\text { tervention compared with oral functional training }\end{array}$ \\
\hline Knowles 1988 & $\begin{array}{l}\text { Not a supportive intervention in nutritional care; intervention given to outpatients, therefore par- } \\
\text { ticipants would have been given individual advice on taking ONS; intervention was tailored and } \\
\text { targeted at increasing intake by } 50 \% \text { above normal }\end{array}$
\end{tabular}

Krondl 1999

Not a supportive intervention in nutritional care; intervention given to outpatients, therefore participants would have been given individual advice on taking ONS

\begin{tabular}{|c|c|}
\hline Kruizenga 2004 & Not a RCT \\
\hline Kuhlmann 1997 & $\begin{array}{l}\text { Not a supportive intervention in nutritional care; intervention given to outpatients, therefore par- } \\
\text { ticipants would have been given individual advice on taking ONS }\end{array}$ \\
\hline Kwok 2001 & $\begin{array}{l}\text { Not a supportive intervention in nutritional care; intervention was described, however no clear or- } \\
\text { ganisational component to the intervention was given }\end{array}$ \\
\hline Kwok 2012 & $\begin{array}{l}\text { Not a supportive intervention in nutritional care; examined whether dietary interventions promot- } \\
\text { ing intakes of fruit, vegetable, fish and lower salt, intake were effective in preventing cognitive de- } \\
\text { cline in older people }\end{array}$ \\
\hline Lauque 2000 & $\begin{array}{l}\text { Not a supportive intervention in nutritional care; intervention was described, however no clear or- } \\
\text { ganisational component to the intervention was given; intervention not identical for all partici- } \\
\text { pants, variety of oral nutritional support offered and dietitian visited sites regularly to direct prod- } \\
\text { uct distribution and intake, hence likely tailoring }\end{array}$ \\
\hline Lauque 2004 & $\begin{array}{l}\text { Not a supportive intervention in nutritional care; intervention not identical for all participants, vari- } \\
\text { ety of ONS offered ranging between 300-500 kcal therefore likely tailoring }\end{array}$ \\
\hline Lawson 2000 & Not a RCT \\
\hline Le Cornu 2000 & $\begin{array}{l}\text { Not a supportive intervention in nutritional care; intervention given to outpatients, therefore par- } \\
\text { ticipants would have been given individual advice on taking ONS }\end{array}$ \\
\hline Lee 2013 & $\begin{array}{l}\text { Participants were selected for the intervention after group allocation on the basis of their nutrition- } \\
\text { al status rather than before intervention, or by restricting the inclusion to malnourished partici- } \\
\text { pants only }\end{array}$ \\
\hline Leon 2001 & Not a supportive intervention in nutritional care; individualised intervention \\
\hline Leon 2006 & Not a supportive intervention in nutritional care; individualised intervention \\
\hline Locher 2011 & Not a supportive intervention in nutritional care; dietary advice intervention \\
\hline
\end{tabular}




\begin{tabular}{|c|c|}
\hline Study & Reason for exclusion \\
\hline MacFie 2000 & $\begin{array}{l}\text { Not a supportive intervention in nutritional care; intervention given initially to outpatients, there- } \\
\text { fore participants would have been given individual advice on taking ONS }\end{array}$ \\
\hline Mamhidir 2007 & Not an RCT \\
\hline Manders 2006 & $\begin{array}{l}\text { Not a supportive intervention in nutritional care; intervention was described, however no clear or- } \\
\text { ganisational component to the intervention was given }\end{array}$ \\
\hline McEvoy 1982 & $\begin{array}{l}\text { Not a supportive intervention in nutritional care; intervention was described, however no clear or- } \\
\text { ganisational component to the intervention was given }\end{array}$ \\
\hline McMurdo 2009 & $\begin{array}{l}\text { Not a supportive intervention in nutritional care; intervention given to participants on discharge } \\
\text { from hospital, therefore would have been given individual advice on taking ONS }\end{array}$ \\
\hline Moretti 2009 & $\begin{array}{l}\text { Not a supportive intervention in nutritional care; intervention was given to outpatients, therefore } \\
\text { participants would have been given individual advice on taking ONS }\end{array}$ \\
\hline Navrátilová 2007 & $\begin{array}{l}\text { Not a supportive intervention in nutritional care; intervention was described, however no clear or- } \\
\text { ganisational component to the intervention was given }\end{array}$ \\
\hline Nayel 1992 & $\begin{array}{l}\text { Not a supportive intervention in nutritional care; ONS tailored/individually prescribed according to } \\
\text { requirements (deficit between requirements and intake) }\end{array}$ \\
\hline Olin 1996 & Not a RCT \\
\hline Otte 1989 & $\begin{array}{l}\text { Not a supportive intervention in nutritional care; intervention given to community-dwelling partici- } \\
\text { pants, therefore would have been given individual advice on taking ONS }\end{array}$ \\
\hline Payette 2002 & $\begin{array}{l}\text { Not a supportive intervention in nutritional care; intervention included individualised dietary coun } \\
\text { selling }\end{array}$ \\
\hline
\end{tabular}

Not a supportive intervention in nutritional care; intervention given to participants on discharge
from hospital, therefore would have been given individual advice on taking ONS

\begin{tabular}{ll}
\hline Rana 1992 & $\begin{array}{l}\text { Not a supportive intervention in nutritional care; intervention not identical for all participants; par- } \\
\text { ticipants were allowed to consume ONSat will hence not provided in controlled, routine fashion }\end{array}$ \\
\hline Richeson \& Neil 2004 & Not a RCT; quasi-experimental time series \\
\hline Roberts 2013 & Not a RCT; the protocol for a controlled trial \\
\hline Robinson 2002 & Not a RCT \\
\hline Rosendahl 2006 & able to extract specific effect of nutrition component \\
\hline Roy 2006 & Not randomised control trial; quasi experimental design with an untreated usual care \\
\hline Rypkema 2004 & Not a RCT \\
\hline Saudny-Unterberger 1997 & Nuirements intervention, therefore un- \\
\hline Shinnar 1983 & Not a RCT; observational study \\
\hline Simmons 2004 & Not a RCT; participants allocated according to ability to respond to individualised assistance \\
\hline \hline
\end{tabular}




\begin{tabular}{ll}
\hline Study & Reason for exclusion \\
\hline Smedley 2004 & $\begin{array}{l}\text { Not a supportive intervention in nutritional care; intervention not the same for all participants; par- } \\
\text { ticipants encouraged to consume oral nutritional supplements at will hence not provided in con- } \\
\text { trolled, routine fashion }\end{array}$
\end{tabular}

\begin{tabular}{|c|c|}
\hline Somanchi 2011 & Not a RCT \\
\hline Soneff 1994 & $\begin{array}{l}\text { Not a supportive intervention in nutritional care; outcomes reported at facility level, not partici- } \\
\text { pant level }\end{array}$ \\
\hline Southgate 2010 & Not a supportive intervention in nutritional care; personalised dietetic intervention \\
\hline Starke 2011 & Not a supportive intervention in nutritional care; individualised intervention \\
\hline Stauffer 1986 & Not a RCT: a prospective observational study \\
\hline Steiner 2003 & $\begin{array}{l}\text { Not a supportive intervention in nutritional care; intervention was given to outpatients, therefore } \\
\text { participants would have been given individual advice on taking ONS }\end{array}$ \\
\hline Stotts 2009 & $\begin{array}{l}\text { Not a supportive intervention in nutritional care; intervention involved administration of supple- } \\
\text { mental fluid }\end{array}$ \\
\hline Teixido-Planas 2005 & $\begin{array}{l}\text { Not a supportive intervention in nutritional care; intervention was given to outpatients, therefore } \\
\text { participants would have been given individual advice on taking ONS }\end{array}$ \\
\hline Tkatch 1992 & $\begin{array}{l}\text { Not a supportive intervention in nutritional care; intervention was described, however no clear or- } \\
\text { ganisational component to the intervention was given }\end{array}$ \\
\hline Vetter 1992 & $\begin{array}{l}\text { Not a supportive intervention in nutritional care; multicomponent intervention; difficult to extract } \\
\text { specific effect of nutrition component; included dietary advice }\end{array}$ \\
\hline Vlaming 2001 & $\begin{array}{l}\text { Not a supportive intervention in nutritional care; intervention was described, however no clear or- } \\
\text { ganisational component to the intervention was given }\end{array}$ \\
\hline Watanabe 2010 & Not a RCT; appears to be a matched cohort \\
\hline Williams 1989 & Not a RCT \\
\hline Wong 2010 & Not a RCT \\
\hline Woo 1994 & $\begin{array}{l}\text { Not a supportive intervention in nutritional care; intervention was given on hospital discharge, } \\
\text { therefore participants would have been given individual advice on taking ONS }\end{array}$ \\
\hline Wouters-Wesseling 2002 & $\begin{array}{l}\text { Not a supportive intervention in nutritional care; intervention was described, however no clear or- } \\
\text { ganisational component to the intervention was given }\end{array}$ \\
\hline Wright 2006 & Not a RCT; quasi-experimental \\
\hline WY Lin 2010 & $\begin{array}{l}\text { Not a supportive intervention in nutritional care; multicomponent intervention; difficult to extract } \\
\text { specific effect of nutrition component; the presence of a dietitian in the multidiciplinary team was } \\
\text { the only difference between the two groups }\end{array}$ \\
\hline Yamaguchi 1998 & $\begin{array}{l}\text { Not a supportive intervention in nutritional care; intervention was given to outpatients, therefore } \\
\text { participants would have been given individual advice on taking ONS }\end{array}$ \\
\hline Young 2004 & Not a RCT \\
\hline
\end{tabular}




\begin{tabular}{ll}
\hline Study & Reason for exclusion \\
\hline Ödlund Olin 2003 & Not a RCT \\
\hline
\end{tabular}

ONS: oral nutritional supplement; RCT: randomised controlled trial

Characteristics of studies awaiting assessment [ordered by study ID]

\begin{tabular}{ll} 
Allen $\mathbf{2 0 1 4}$ & RCT \\
\hline Methods & $\begin{array}{l}\text { Participants with long-standing cognitive impairment in hospital or living in a residential care } \\
\text { home }\end{array}$ \\
\hline Interventions & $\begin{array}{l}\text { Oral nutritional supplement drink provided } 3 \text { times a day in a glass/beaker or consumed through a } \\
\text { straw inserted directly into the container }\end{array}$ \\
\hline Outcomes & Amount of nutritional supplement drink consumed \\
\hline Notes & Full data extraction has not yet been undertaken and will be completed at the next update \\
\hline
\end{tabular}

\section{Borges 2003}

Methods

\section{Participants}

Interventions

\section{Outcomes}

Notes Requires translation, unable to locate abstract

Burns 1998

Methods

Participants

Interventions

Outcomes

Notes Requires translation, unable to locate abstract 
Deutz 2016 (Continued)

Participants

Older (> 65 years), malnourished adults hospitalised for congestive heart failure, acute myocardial infarction, pneumonia or chronic obstructive pulmonary disease

\begin{tabular}{ll}
\hline Interventions & Standard-of-care plus a high-protein oral nutritional supplement or a placebo supplement \\
\hline Outcomes & $\begin{array}{l}\text { Primary composite endpoint: } 90 \text {-day postdischarge incidence of death or nonelective readmission; } \\
\text { other endpoints: } 30 \text { - and } 60 \text {-day postdischarge incidence of death or readmission, length of stay, } \\
\text { malnourishment class ()SGA, body weight, and ADL }\end{array}$
\end{tabular}

Notes

Full data extraction has not yet been undertaken and will be completed at the next update

\section{Ekinci 2016}

\begin{tabular}{ll}
\hline Methods & RCT \\
\hline Participants & Older female participants with a hip fracture \\
\hline Interventions & $\begin{array}{l}\text { The intervention group received an enteral product containing } 3 \text { g calcium beta-hydroxy-be- } \\
\text { ta-methylbutyrate, } 1000 \text { IU vitamin D and } 36 \text { g protein, in addition to standard postoperative nutri- } \\
\text { tion. The control group received standard postoperative nutrition }\end{array}$ \\
\hline Outcomes & Wound-healing period, shortening of immobilisation period, muscle strength, BMI \\
\hline Notes & Full data extraction has not yet been undertaken and will be completed at the next update \\
\hline
\end{tabular}

\section{ISRCTN04327195}

\begin{tabular}{ll}
\hline Methods & RCT \\
\hline Participants & Undernourished geriatric inpatients \\
\hline Interventions & $\begin{array}{l}\text { Intervention group: energy dense, small volume oral nutritional supplements; control group: forti- } \\
\text { fied foods }\end{array}$ \\
\hline Outcomes & $\begin{array}{l}\text { Primary outcome measure: number of participants achieving an extra intake of } 450 \text { kcal per day; } \\
\text { secondary outcome measures: recommended energy and protein intakes, length of hospital stay, } \\
\text { antibiotic usage }\end{array}$ \\
\hline Notes & Retrospectively registered; trial end date: 15 May 2010 \\
\hline
\end{tabular}

\begin{tabular}{ll}
\hline Methods & RCT \\
\hline Participants & Malnutrition in the elderly \\
\hline Interventions & $\begin{array}{l}\text { Standard dietary care versus a high-energy supplement versus a high-energy supplement plus mi- } \\
\text { cronutrients }\end{array}$ \\
\hline Outcomes & $\begin{array}{l}\text { Primary outcome measure: nutrient intake; secondary outcome measures: gastro-intestinal toler- } \\
\text { ance, product compliance, appetite, anthropometry (weight and BMI), muscle function, measured }\end{array}$ \\
\hline
\end{tabular}


ISRCTN96923961 (Continued)

by hand grip dynamometry, quality of life, measured using EuroQol EQ-5D questionnaire, blood lipids and micronutrients, safety, falls assessment measured using Berg Balance Scale

Notes Retrospectively registered; trial end date: 30 December 2007

\section{Jobse 2015}

\begin{tabular}{ll}
\hline Methods & RCT \\
\hline Participants & Nursing home residents with malnutrition or at risk of malnutrition \\
\hline Interventions & $\begin{array}{l}\text { Intervention group received } 2 \times 125 \mathrm{~mL} \text { oral nutritional supplements for } 12 \text { weeks, and the control } \\
\text { group received usual care }\end{array}$ \\
\hline Outcomes & Body weight change, BMI, upper arm and calf-circumferences, MNA score \\
\hline Notes & Full data extraction has not yet been undertaken and will be completed at the next update \\
\hline
\end{tabular}

\section{Lee 2015}

\begin{tabular}{ll}
\hline Methods & RCT \\
\hline Participants & Older people living in a nursing home \\
\hline Interventions & $\begin{array}{l}\text { Each participant in the intervention group received a } 50 \text { g/day soy-protein-based nutritional sup- } \\
\text { plement when he/she was rated as undernourished; all participants including those who were in } \\
\text { the control group received the same normal meals and a light afternoon snack daily }\end{array}$ \\
\hline Outcomes & Handgrip strength, Barthel index, anthropometric and biochemical indicators \\
\hline Notes & Full data extraction has not yet been undertaken and will be completed at the next update
\end{tabular}

Leslie 2013

\begin{tabular}{ll}
\hline Methods & Cluster-randomised trial in 21 residential care homes \\
\hline Participants & Undernourished residents with a BMI $<18.5 \mathrm{~kg} / \mathrm{m}^{2}$ \\
\hline Interventions & Enrichment of meals to increase energy density \\
\hline Outcomes & Nutritional intake, body weight, MUAC, BMI, mortality \\
\hline Notes & Full data extraction has not yet been undertaken and will be completed at the next update \\
\hline
\end{tabular}

Luna-Ramos 2016

\begin{tabular}{ll}
\hline Methods & RCT \\
\hline
\end{tabular}


Luna-Ramos 2016 (Continued)

Participants Elderly fragile, hospitalised participants

\begin{tabular}{ll}
\hline Interventions & Polymeric diet versus standard diet \\
\hline Outcomes & Nutritional status, BMI, body weight \\
\hline Notes & Full data extraction has not yet been undertaken and will be completed at the next update \\
\hline
\end{tabular}

\section{Madigan 1994}

\begin{tabular}{ll}
\hline Methods & Unclear \\
\hline Participants & Elderly participants with fractured neck of femur \\
\hline Interventions & $\begin{array}{l}\text { Oral feed with protein and energy vs normal ward diet, followed up for } 3 \text { months post hospital dis- } \\
\text { charge }\end{array}$ \\
\hline Outcomes & Mortality, length of hospital stay, postoperative functional status, dietary intake, compliance \\
\hline Notes & Unable to locate dissertation \\
\hline
\end{tabular}

Moore 2010

\begin{tabular}{ll}
\hline Methods & RCT \\
\hline Participants & Older people with dementia living in a residential care home and an assisted living facility \\
\hline Interventions & $\begin{array}{l}\text { A 25-min activity offered } 30 \text { min before meal times (aiming to reduce apathy and agitation and to } \\
\text { increase eating ability and intake }\end{array}$ \\
\hline Outcomes & Apathy, agitation, eating ability, dietary intake \\
\hline Notes & Full data extraction has not yet been undertaken and will be completed at the next update \\
\hline
\end{tabular}

\section{Parsons 2016}

\begin{tabular}{ll}
\hline Methods & RCT \\
\hline Participants & Malnourished, care home residents \\
\hline Interventions & Oral nutritional supplements or dietary advice \\
\hline Outcomes & Health-related quality of life, nutritional intake \\
\hline Notes & Full data extraction has not yet been undertaken and will be completed at the next update \\
\hline
\end{tabular}


Pouyssegur 2015

\begin{tabular}{ll}
\hline Methods & A multicentre RCT \\
\hline Participants & Malnourished older adults living in nursing homes \\
\hline Interventions & $\begin{array}{l}\text { In addition to usual meals, the provision of eight cookies (30 kcals and } 1.44 \text { g protein) throughout } \\
\text { the day }\end{array}$ \\
\hline Outcomes & Body weight, appetite, occurrence of pressure ulcers, diarrhoea \\
\hline Notes & Full data extraction has not yet been undertaken and will be completed at the next update \\
\hline
\end{tabular}

Scorer 1990

\section{Methods}

Participants

Interventions

\section{Outcomes}

Notes Unable to locate paper

\section{Simmons 2013}

\begin{tabular}{ll}
\hline Methods & RCT \\
\hline Participants & People living in residential care homes \\
\hline Interventions & Staff training to improve feeding assistance \\
\hline Outcomes & Mealtime feeding assistance, body weight \\
\hline Notes & Full data extraction has not yet been undertaken and will be completed at the next update \\
\hline
\end{tabular}

Simmons 2015

\begin{tabular}{ll}
\hline Methods & RCT \\
\hline Participants & Long-stay residents with orders for nutrition supplementation \\
\hline Interventions & $\begin{array}{l}\text { Usual care control group verus an oral liquid nutrition supplement intervention group, or a snack } \\
\text { intervention group }\end{array}$ \\
\hline Outcomes & $\begin{array}{l}\text { Body weight, food, beverage and supplement intake and the amount of staff time spent providing } \\
\text { assistance, cost-effectiveness }\end{array}$ \\
\hline Notes & Full data extraction has not yet been undertaken and will be completed at the next update \\
\hline
\end{tabular}


Stelten 2015

\begin{tabular}{ll}
\hline Methods & Single-blind RCT \\
\hline Participants & Acutely ill elderly participants admitted to hospital \\
\hline Interventions & Protein-enriched bread and drinking yoghourt \\
\hline Outcomes & Protein intake \\
\hline Notes & Full data extraction has not yet been undertaken and will be completed at the next update \\
\hline
\end{tabular}

Stow 2015

\begin{tabular}{ll}
\hline Methods & Cluster-RCT \\
\hline Participants & Care home residents with or at risk of malnutrition \\
\hline Interventions & Standard care, food-based intervention or oral nutritional supplement intervention \\
\hline Outcomes & $\begin{array}{l}\text { Anthropometry, dietary intake, healthcare resource usage and participant-reported outcome mea- } \\
\text { sures }\end{array}$ \\
\hline Notes & Registered trial: ISRCTN38047922 \\
& Full data extraction has not yet been undertaken and will be completed at the next update \\
\hline
\end{tabular}

Sutton 2006

\section{Methods}

\section{Participants}

Interventions

\section{Outcomes}

Notes $\quad$ Unable to locate paper

\section{Turano 1999}

\section{Methods}

Participants

Interventions

\section{Outcomes}

\section{Notes}

Requires translation 
White 1999

\section{Methods}

Participants

Interventions

\section{Outcomes}

\begin{tabular}{ll}
\hline Notes & Unable to locate paper \\
\hline
\end{tabular}

\section{Zhong 2016}

\begin{tabular}{ll}
\hline Methods & RCT and economic evaluation \\
\hline Participants & Malnourished older hospitalised participants \\
\hline Interventions & $\begin{array}{l}\text { Nutrient-dense ONS, containing high protein and beta-hydroxy-beta-methylbutyrate versus place- } \\
\text { bo }\end{array}$ \\
\hline Outcomes & $\begin{array}{l}\text { Health-care costs, measured as the product of resource use and per unit cost, quality-adjusted life- } \\
\text { years (QALYs), life-years saved and the incremental cost-effectiveness ratio }\end{array}$ \\
\hline Notes & Full data extraction has not yet been undertaken and will be completed at the next update \\
\hline
\end{tabular}

ADL: activities of daily living; BMI: body mass index; MNA: Mini Nutritional Assessment; MUAC: mid upper-arm circumference; ONS: oral nutritional supplement; SGA: subjective global assessment

\section{DATA AND ANALYSES}

\section{Comparison 1. Supportive interventions for enhancing dietary intake versus comparators}

\begin{tabular}{|c|c|c|c|c|}
\hline Outcome or subgroup title & No. of studies & $\begin{array}{l}\text { No. of partici- } \\
\text { pants }\end{array}$ & Statistical method & Effect size \\
\hline $\begin{array}{l}1 \text { No. of participants with complica- } \\
\text { tions }\end{array}$ & 5 & 4702 & $\begin{array}{l}\text { Risk Ratio (M-H, Random, 95\% } \\
\text { Cl) }\end{array}$ & $1.11[0.86,1.42]$ \\
\hline $\begin{array}{l}1.1 \text { Changes to the organisation of } \\
\text { nutritional care }\end{array}$ & 3 & 624 & $\begin{array}{l}\text { Risk Ratio (M-H, Random, 95\% } \\
\mathrm{Cl} \text { ) }\end{array}$ & $1.12[0.76,1.67]$ \\
\hline $\begin{array}{l}\text { 1.2 Modification of meal profile or } \\
\text { pattern }\end{array}$ & 1 & 63 & $\begin{array}{l}\text { Risk Ratio (M-H, Random, 95\% } \\
\mathrm{Cl} \text { ) }\end{array}$ & $0.59[0.06,6.14]$ \\
\hline $\begin{array}{l}1.3 \text { Additional supplementation of } \\
\text { meals }\end{array}$ & 1 & 4015 & $\begin{array}{l}\text { Risk Ratio (M-H, Random, 95\% } \\
\text { Cl) }\end{array}$ & $1.14[1.02,1.28]$ \\
\hline 2 Nutritional status (weight change) & 17 & 2024 & $\begin{array}{l}\text { Mean Difference (IV, Random, } \\
95 \% \mathrm{CI})\end{array}$ & $0.62[0.21,1.02]$ \\
\hline
\end{tabular}




\begin{tabular}{|c|c|c|c|c|}
\hline Outcome or subgroup title & No. of studies & $\begin{array}{l}\text { No. of partici- } \\
\text { pants }\end{array}$ & Statistical method & Effect size \\
\hline $\begin{array}{l}2.1 \text { Changes to the organisation of } \\
\text { nutritional care }\end{array}$ & 6 & 1140 & $\begin{array}{l}\text { Mean Difference (IV, Random, } \\
95 \% \mathrm{CI})\end{array}$ & $0.09[-0.26,0.45]$ \\
\hline $\begin{array}{l}2.2 \text { Changes to the feeding environ- } \\
\text { ment }\end{array}$ & 1 & 39 & $\begin{array}{l}\text { Mean Difference (IV, Random, } \\
95 \% \mathrm{CI})\end{array}$ & $-0.43[-2.11,1.25]$ \\
\hline $\begin{array}{l}\text { 2.3 Modification of meal profile or } \\
\text { pattern }\end{array}$ & 5 & 253 & $\begin{array}{l}\text { Mean Difference (IV, Random, } \\
95 \% \mathrm{CI})\end{array}$ & $1.16[0.41,1.92]$ \\
\hline $\begin{array}{l}\text { 2.4 Additional supplementation of } \\
\text { meals }\end{array}$ & 4 & 475 & $\begin{array}{l}\text { Mean Difference (IV, Random, } \\
95 \% \mathrm{CI})\end{array}$ & $0.90[0.41,1.38]$ \\
\hline $\begin{array}{l}2.5 \text { Congregate and home meal de- } \\
\text { livery systems }\end{array}$ & 1 & 117 & $\begin{array}{l}\text { Mean Difference (IV, Random, } \\
95 \% \mathrm{CI})\end{array}$ & $2.90[1.00,4.80]$ \\
\hline 3 Hospitalisation & 5 & 667 & $\begin{array}{l}\text { Mean Difference (IV, Random, } \\
95 \% \mathrm{CI})\end{array}$ & $-0.48[-2.56,1.59]$ \\
\hline $\begin{array}{l}\text { 3.1 Changes to the organisation of } \\
\text { nutritional care }\end{array}$ & 3 & 515 & $\begin{array}{l}\text { Mean Difference (IV, Random, } \\
95 \% \mathrm{CI})\end{array}$ & $-2.08[-6.75,2.58]$ \\
\hline $\begin{array}{l}3.2 \text { Modification of meal profile or } \\
\text { pattern }\end{array}$ & 1 & 81 & $\begin{array}{l}\text { Mean Difference (IV, Random, } \\
95 \% \mathrm{CI})\end{array}$ & $0.0[-3.48,3.48]$ \\
\hline $\begin{array}{l}\text { 3.3 Additional supplementation of } \\
\text { meals }\end{array}$ & 1 & 71 & $\begin{array}{l}\text { Mean Difference (IV, Random, } \\
95 \% \mathrm{CI})\end{array}$ & $0.20[-2.26,2.66]$ \\
\hline 4 All-cause mortality & 12 & 6683 & $\begin{array}{l}\text { Risk Ratio (M-H, Random, 95\% } \\
\mathrm{Cl})\end{array}$ & $0.78[0.66,0.92]$ \\
\hline $\begin{array}{l}\text { 4.1 Changes to the organisation of } \\
\text { nutritional care }\end{array}$ & 4 & 1237 & $\begin{array}{l}\text { Risk Ratio (M-H, Random, 95\% } \\
\mathrm{Cl})\end{array}$ & $0.71[0.52,0.97]$ \\
\hline $\begin{array}{l}\text { 4.2 Changes to the feeding environ- } \\
\text { ment }\end{array}$ & 1 & 20 & $\begin{array}{l}\text { Risk Ratio (M-H, Random, 95\% } \\
\mathrm{Cl})\end{array}$ & $3.00[0.14,65.90]$ \\
\hline $\begin{array}{l}4.3 \text { Modification of meal profile or } \\
\text { pattern }\end{array}$ & 2 & 150 & $\begin{array}{l}\text { Risk Ratio (M-H, Random, 95\% } \\
\mathrm{Cl})\end{array}$ & $1.04[0.15,7.22]$ \\
\hline $\begin{array}{l}\text { 4.4 Additional supplementation of } \\
\text { meals }\end{array}$ & 4 & 5073 & $\begin{array}{l}\text { Risk Ratio (M-H, Random, 95\% } \\
\text { Cl) }\end{array}$ & $0.77[0.58,1.02]$ \\
\hline $\begin{array}{l}4.5 \text { Congregate and home meal de- } \\
\text { livery systems }\end{array}$ & 1 & 203 & $\begin{array}{l}\text { Risk Ratio (M-H, Random, 95\% } \\
\mathrm{Cl} \text { ) }\end{array}$ & $0.33[0.09,1.18]$ \\
\hline
\end{tabular}


Analysis 1.1. Comparison 1 Supportive interventions for enhancing dietary intake versus comparators, Outcome 1 No. of participants with complications.

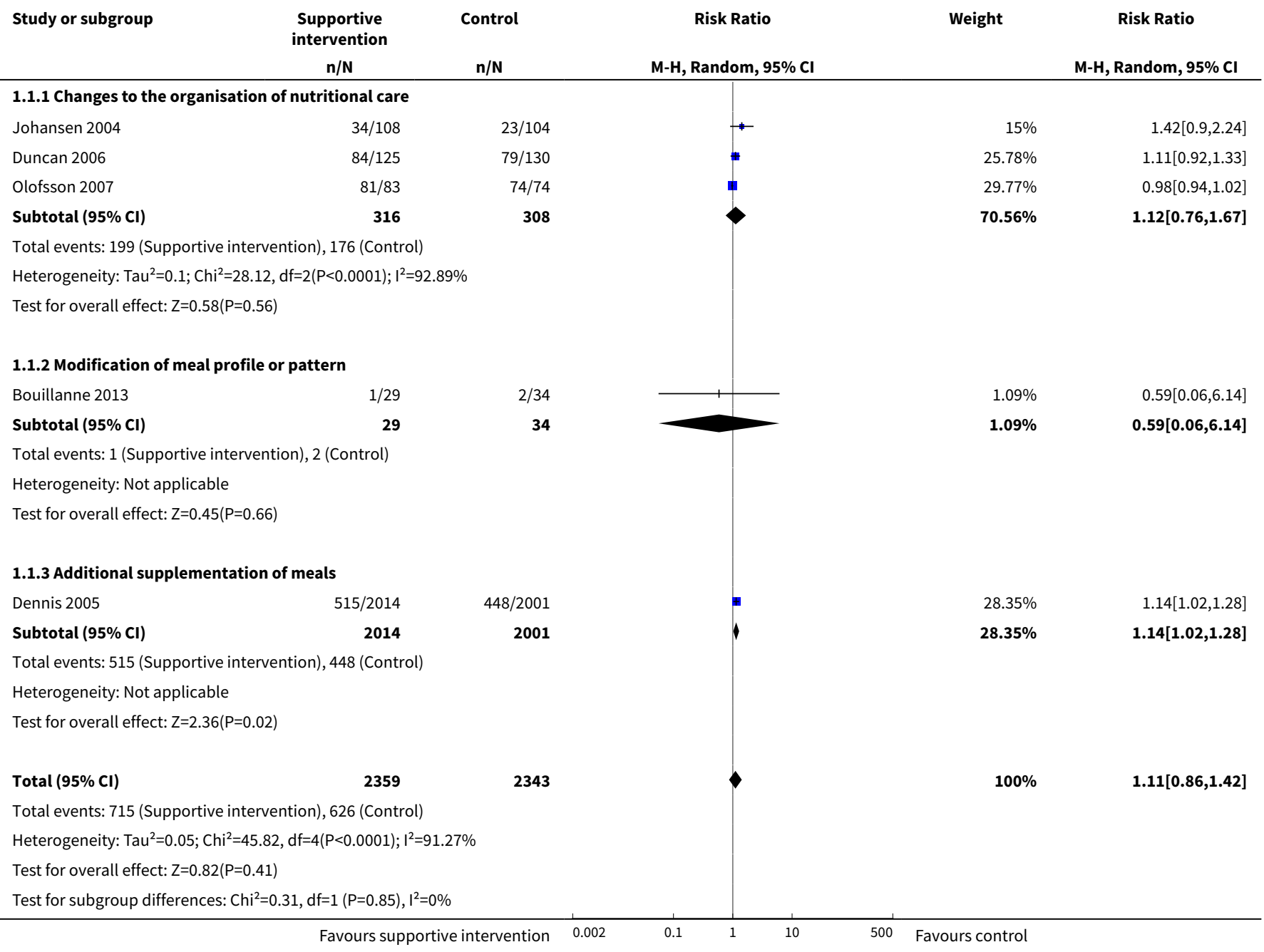

Analysis 1.2. Comparison 1 Supportive interventions for enhancing dietary intake versus comparators, Outcome 2 Nutritional status (weight change).

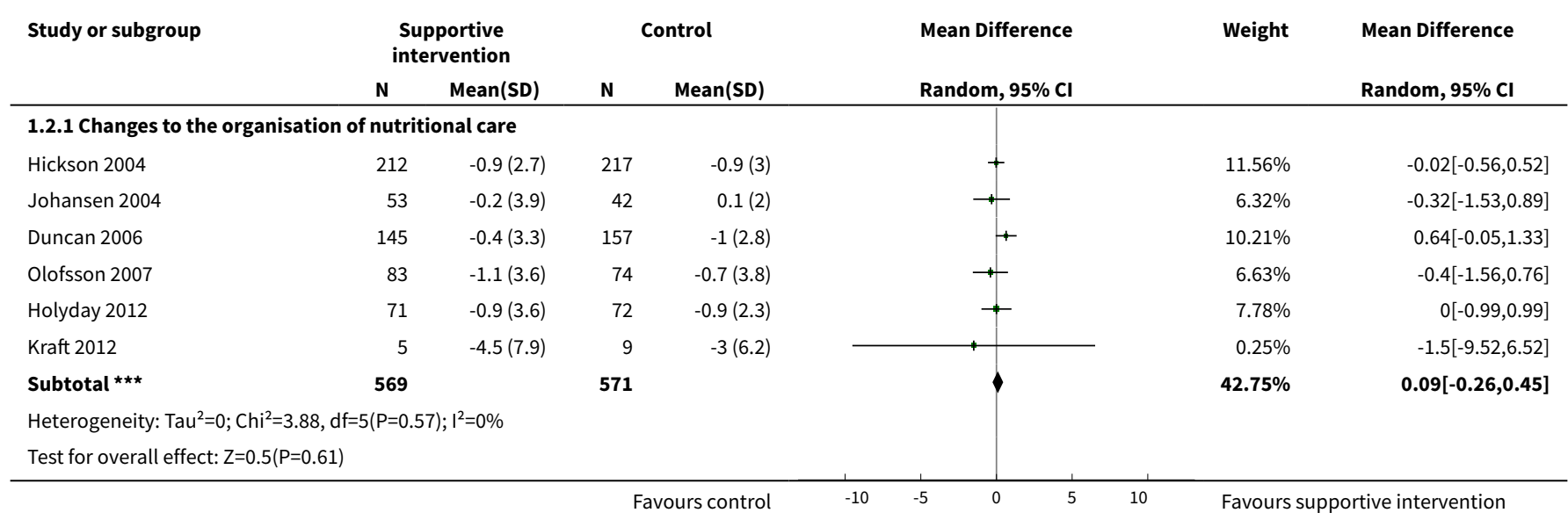




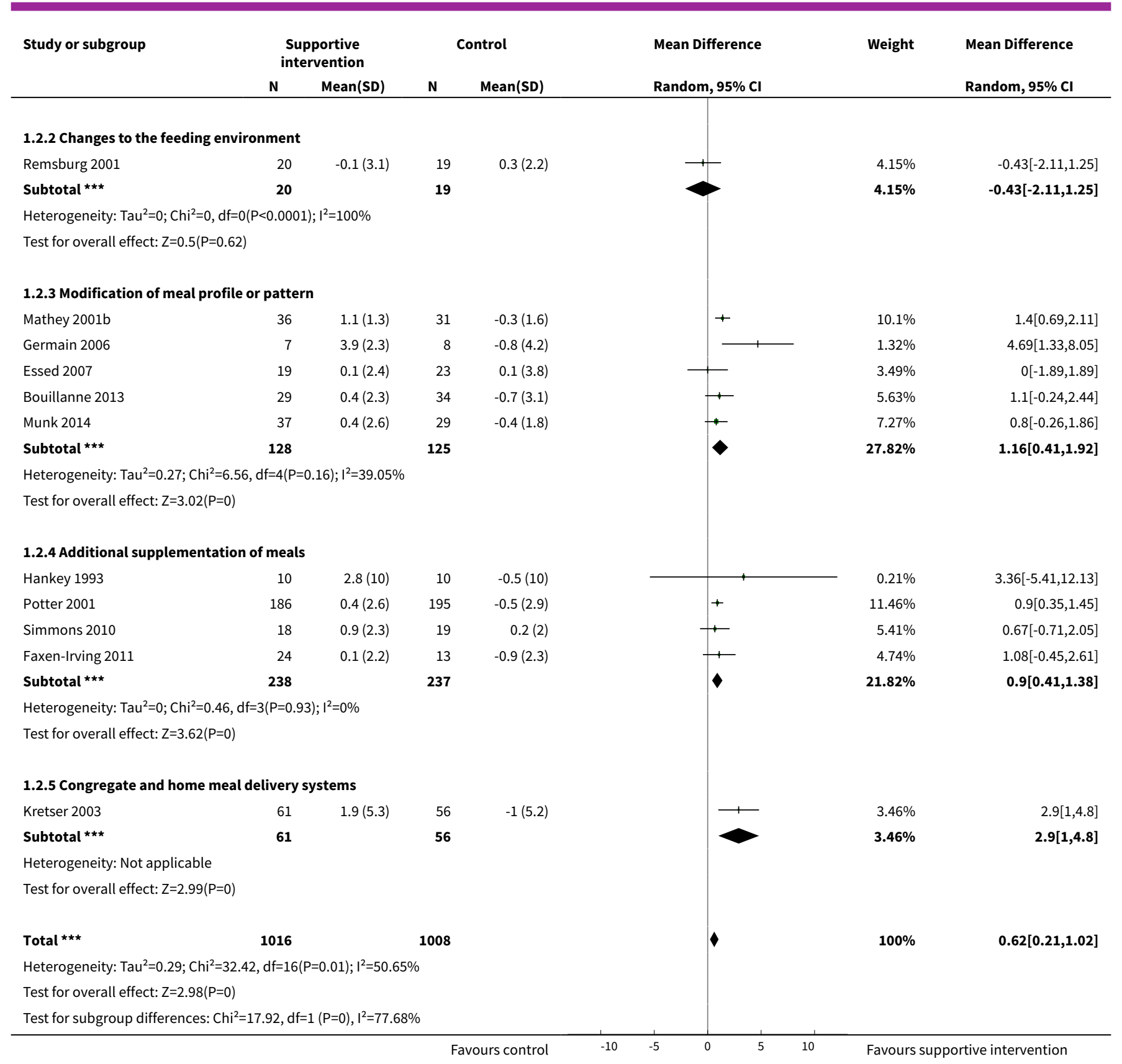

\section{Analysis 1.3. Comparison 1 Supportive interventions for enhancing dietary intake versus comparators, Outcome 3 Hospitalisation.}

\begin{tabular}{|c|c|c|c|c|c|c|c|}
\hline \multirow[t]{2}{*}{ Study or subgroup } & \multicolumn{2}{|c|}{$\begin{array}{c}\text { Supportive } \\
\text { intervention }\end{array}$} & \multicolumn{2}{|c|}{ Control } & \multirow{2}{*}{$\begin{array}{l}\text { Mean Difference } \\
\text { Random, } 95 \% \mathrm{Cl}\end{array}$} & \multirow[t]{2}{*}{ Weight } & \multirow{2}{*}{$\begin{array}{l}\text { Mean Difference } \\
\text { Random, } 95 \% \mathrm{Cl}\end{array}$} \\
\hline & $\mathbf{N}$ & Mean(SD) & $\mathbf{N}$ & Mean(SD) & & & \\
\hline \multicolumn{8}{|c|}{ 1.3.1 Changes to the organisation of nutritional care } \\
\hline Johansen 2004 & 90 & $11.6(8)$ & 82 & $11.5(8)$ & 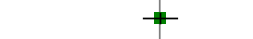 & $28.56 \%$ & $0.1[-2.29,2.49]$ \\
\hline Olofsson 2007 & 102 & $27.4(15.9)$ & 97 & $39.8(41.9)$ & - & $4.87 \%$ & $-12.4[-21.29,-3.51]$ \\
\hline Holyday 2012 & 72 & $13.7(11.8)$ & 72 & $13.5(11)$ & $\rightarrow$ & $18.54 \%$ & $0.2[-3.53,3.93]$ \\
\hline Subtotal $* \star \star$ & 264 & & 251 & & & $51.97 \%$ & $-2.08[-6.75,2.58]$ \\
\hline
\end{tabular}




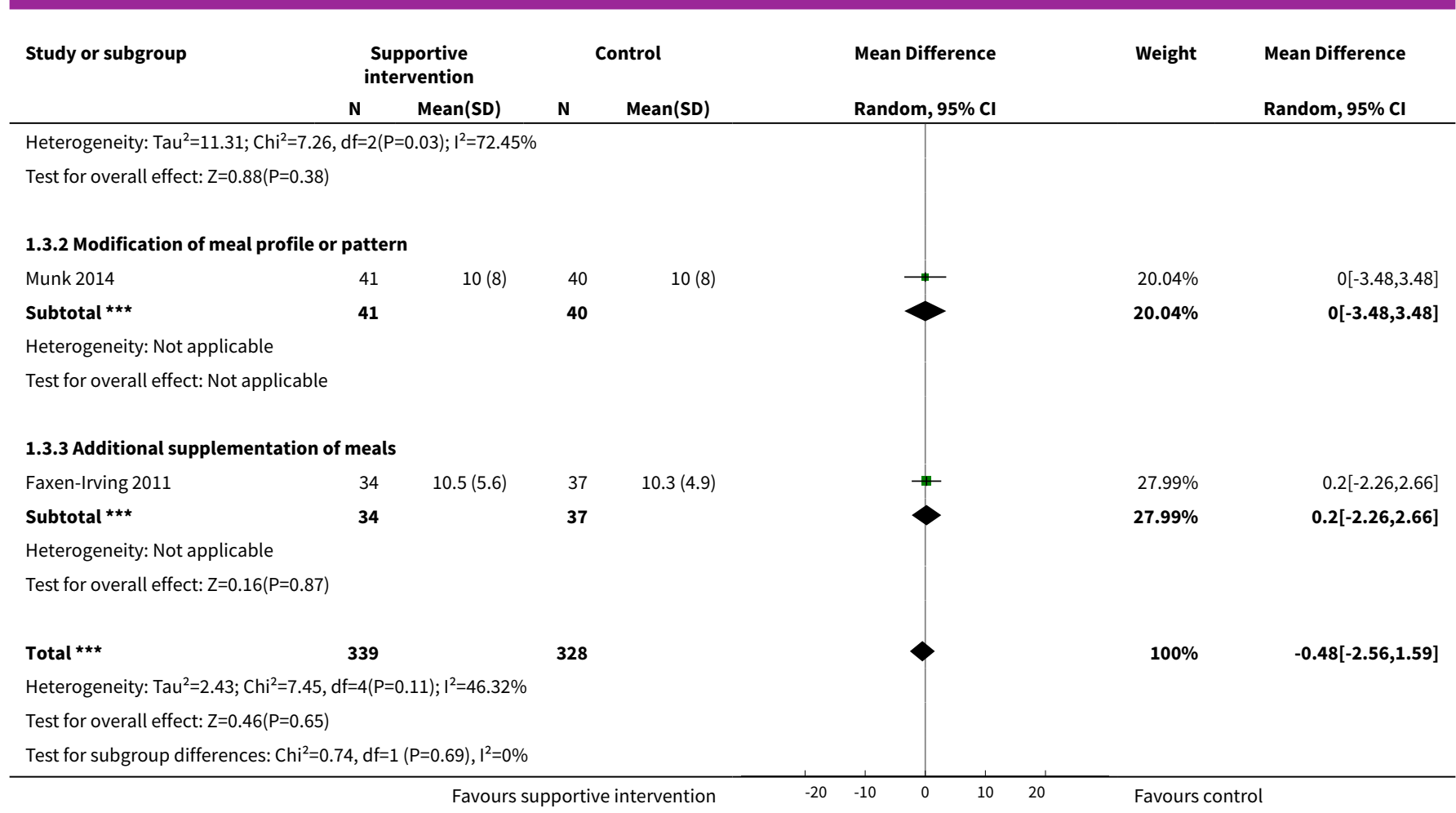

\section{Analysis 1.4. Comparison 1 Supportive interventions for enhancing dietary intake versus comparators, Outcome 4 All-cause mortality.}

\begin{tabular}{|c|c|c|c|c|c|}
\hline Study or subgroup & $\begin{array}{c}\text { Supportive } \\
\text { intervention } \\
n / N \\
\end{array}$ & $\begin{array}{l}\text { Control } \\
\mathbf{n} / \mathbf{N}\end{array}$ & $\begin{array}{c}\text { Risk Ratio } \\
\text { M-H, Random, } 95 \% \mathrm{CI}\end{array}$ & Weight & $\begin{array}{c}\text { Risk Ratio } \\
\text { M-H, Random, } 95 \% \mathrm{Cl}\end{array}$ \\
\hline \multicolumn{6}{|c|}{ 1.4.1 Changes to the organisation of nutritional care } \\
\hline Hickson 2004 & $31 / 292$ & $35 / 300$ & $\rightarrow$ & $11.75 \%$ & $0.91[0.58,1.44]$ \\
\hline Duncan 2006 & $19 / 145$ & $36 / 157$ & *- & $9.74 \%$ & $0.57[0.34,0.95]$ \\
\hline Olofsson 2007 & $9 / 102$ & $13 / 97$ & $\longrightarrow$ & $4.19 \%$ & $0.66[0.29,1.47]$ \\
\hline Subtotal $(95 \% \mathrm{Cl})$ & 611 & 626 & & $26.28 \%$ & $0.71[0.52,0.97]$ \\
\hline \multicolumn{6}{|c|}{ Total events: 60 (Supportive intervention), 88 (Control) } \\
\hline \multicolumn{6}{|c|}{ Heterogeneity: $\mathrm{Tau}^{2}=0 ; \mathrm{Chi}^{2}=2.77, \mathrm{df}=3(\mathrm{P}=0.43) ; \mathrm{I}^{2}=0 \%$} \\
\hline \multicolumn{6}{|c|}{ Test for overall effect: $Z=2.16(P=0.03)$} \\
\hline \multicolumn{6}{|c|}{ 1.4.2 Changes to the feeding environment } \\
\hline Subtotal $(95 \% \mathrm{Cl})$ & 10 & 10 & & $0.3 \%$ & $3[0.14,65.9]$ \\
\hline \multicolumn{6}{|c|}{ Total events: 1 (Supportive intervention), 0 (Control) } \\
\hline \multicolumn{6}{|c|}{ Heterogeneity: Not applicable } \\
\hline \multicolumn{6}{|c|}{ Test for overall effect: $Z=0.7(P=0.49)$} \\
\hline \multicolumn{6}{|c|}{ 1.4.3 Modification of meal profile or pattern } \\
\hline Bouillanne 2013 & $1 / 30$ & $1 / 36$ & & $0.38 \%$ & $1.2[0.08,18.38]$ \\
\hline Munk 2014 & $1 / 44$ & $1 / 40$ & & $0.38 \%$ & $0.91[0.06,14.06]$ \\
\hline Subtotal $(95 \% \mathrm{CI})$ & 74 & 76 & & $0.76 \%$ & $1.04[0.15,7.22]$ \\
\hline Total events: 2 (Supp & on), 2 (Control) & & & & \\
\hline
\end{tabular}




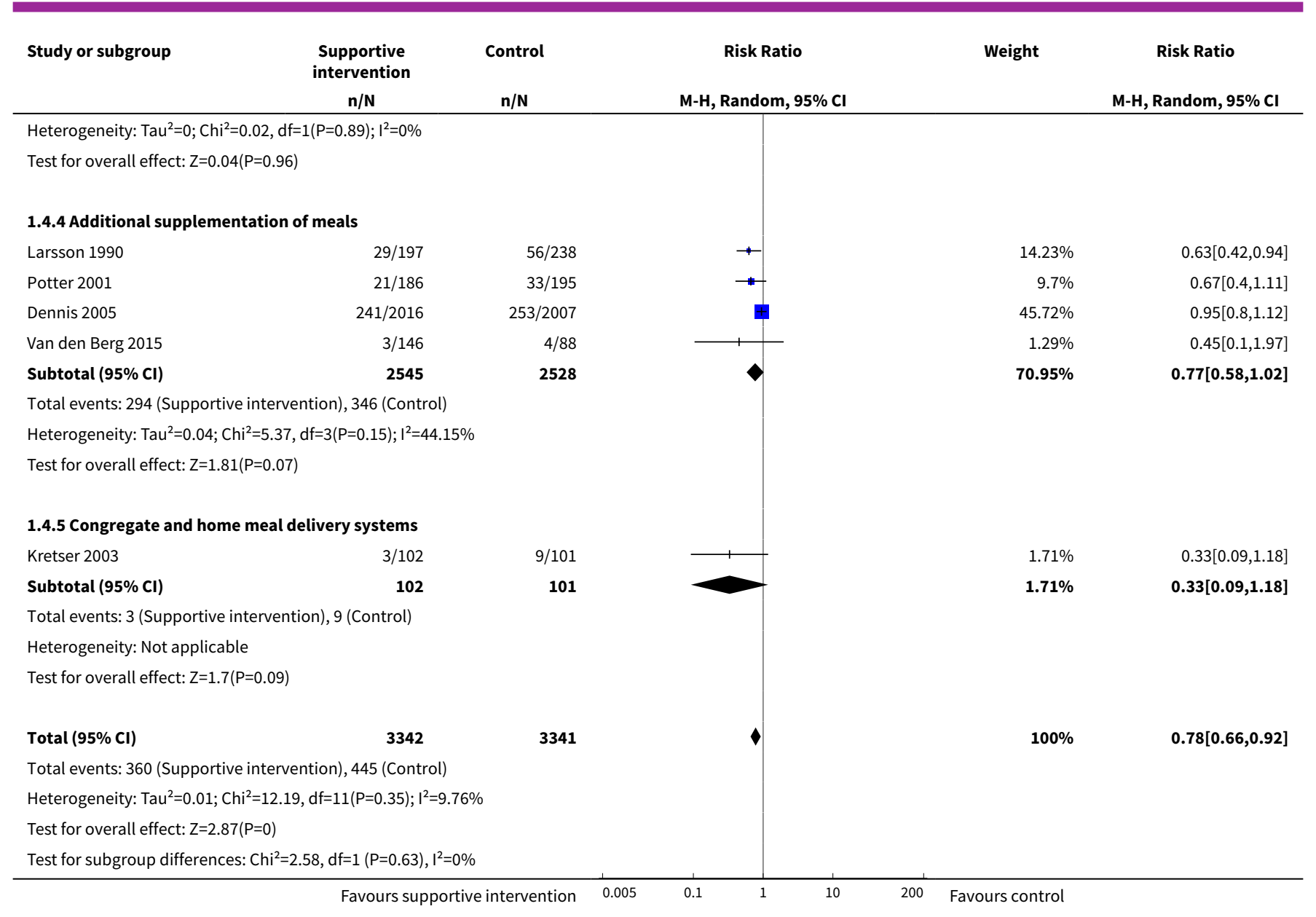

\section{ADDITIONAL TABLES}

\section{Table 1. Intervention subcategories}

\section{Supportive nutritional care in- Examples tervention}

\section{Broad intervention category}

\begin{tabular}{ll}
$\begin{array}{ll}\text { 1. Changes to the organisation of } \\
\text { nutritional care }\end{array}$ & - Use of dietetic or healthcare assistants \\
& - Monitoring and documentation of nutritional care \\
& - Implementation of nutritional care pathways/protocols \\
& - Identification of nutritionally at-risk individuals (e.g. red trays, mandatory nutrition screening) \\
\hline $\begin{array}{l}\text { 2. Changes to the feeding envi- } \\
\text { ronment }\end{array}$ & - Changes to dining arrangements/style/setting \\
& - Protected meal times
\end{tabular}
3. Modification of meal profile or
- Changes to meal pattern (e.g. 5 small meals/day) pattern
- Manipulating energy/nutrient density of foods (e.g. food fortification
- Changes to the taste, flavour, appearance of foods, or a combination 
Table 1. Intervention subcategories (Continued)
4. Additional supplementation of
meals individually prescribed)
5. Congregate and home meal de-
- Home meal delivery systems
livery systems
- Community lunch clubs

- Between-meal snacks, drinks or both

- Supplementation with oral nutritional supplements (e.g. routinely provided to entire ward, not 


\begin{tabular}{|c|c|c|c|c|c|c|c|}
\hline & $\begin{array}{l}\text { Intervention(s) and compara- } \\
\text { tor(s) }\end{array}$ & $\begin{array}{l}\text { Screened/eli- } \\
\text { gible } \\
\text { (N) }\end{array}$ & $\begin{array}{l}\text { Randomised } \\
\text { (N) }\end{array}$ & $\begin{array}{l}\text { ITT } \\
\text { (N) }\end{array}$ & $\begin{array}{l}\text { Finishing tri- } \\
\text { al } \\
\text { (N) }\end{array}$ & $\begin{array}{l}\text { Randomised } \\
\text { finishing trial } \\
(\%)\end{array}$ & Follow-up \\
\hline \multirow{4}{*}{$\begin{array}{l}\text { Barton } 2000^{a 2} \\
\text { (modification of meal profile } \\
\text { or pattern) }\end{array}$} & $\begin{array}{l}\text { I1: reduced portion size, forti- } \\
\text { fied menu }\end{array}$ & - & 13 & - & $\mathrm{b}$ & $70 c$ & 56 days \\
\hline & 12: cooked breakfast & & $\begin{array}{l}\text { (8 not ran- } \\
\text { domised) }\end{array}$ & & & & \\
\hline & $\begin{array}{l}\text { C: normal hospital diet with } \\
\text { usual portion size }\end{array}$ & & 14 & & & & \\
\hline & total: & & $27^{a}$ & & - & - & \\
\hline \multirow{4}{*}{$\begin{array}{l}\text { Beck 2002a1 } \\
\text { (additional supplementation } \\
\text { of meals) }\end{array}$} & $\begin{array}{l}\text { I1: homemade oral supplement } \\
\text { (A) }\end{array}$ & - & - & - & - & - & 2 months \\
\hline & $\begin{array}{l}\text { 12: homemade oral supplement } \\
\text { (B) }\end{array}$ & & & & & & \\
\hline & C: usual diet & & & & & & \\
\hline & total: & & 36 & & - & - & \\
\hline \multirow{3}{*}{$\begin{array}{l}\text { Bouillane } 2013^{a 1} \\
\text { (modification of meal profile } \\
\text { or pattern) }\end{array}$} & I: $78 \%$ protein at lunch & - & 30 & - & 30 & 88 & 6 weeks \\
\hline & $\begin{array}{l}\text { C: usual diet (protein distrib- } \\
\text { uted between meals) }\end{array}$ & & 36 & & 23 & 79 & \\
\hline & total: & & 66 & & 63 & 96 & \\
\hline \multirow[t]{3}{*}{$\begin{array}{l}\text { Bourdel-Marchasson } 2000^{a 3} \\
\text { (additional supplementation } \\
\text { of meals) }\end{array}$} & $\begin{array}{l}\text { I: } 2 \text { oral nutritional supple- } \\
\text { ments }\end{array}$ & & 295 & - & - & - & $\begin{array}{l}15 \text { days or un- } \\
\text { til hospital } \\
\text { discharge }\end{array}$ \\
\hline & C: usual care & & 377 & & & & \\
\hline & total: & & 672 & & - & - & \\
\hline \multirow{2}{*}{$\begin{array}{l}\text { Brouillette1991a1 } \\
\text { (changes to the feeding envi- } \\
\text { ronment) }\end{array}$} & I: osmotherapy + activities & - & 10 & - & 9 & 90 & 4 weeks \\
\hline & C: activities only & & 10 & & 7 & 70 & \\
\hline
\end{tabular}




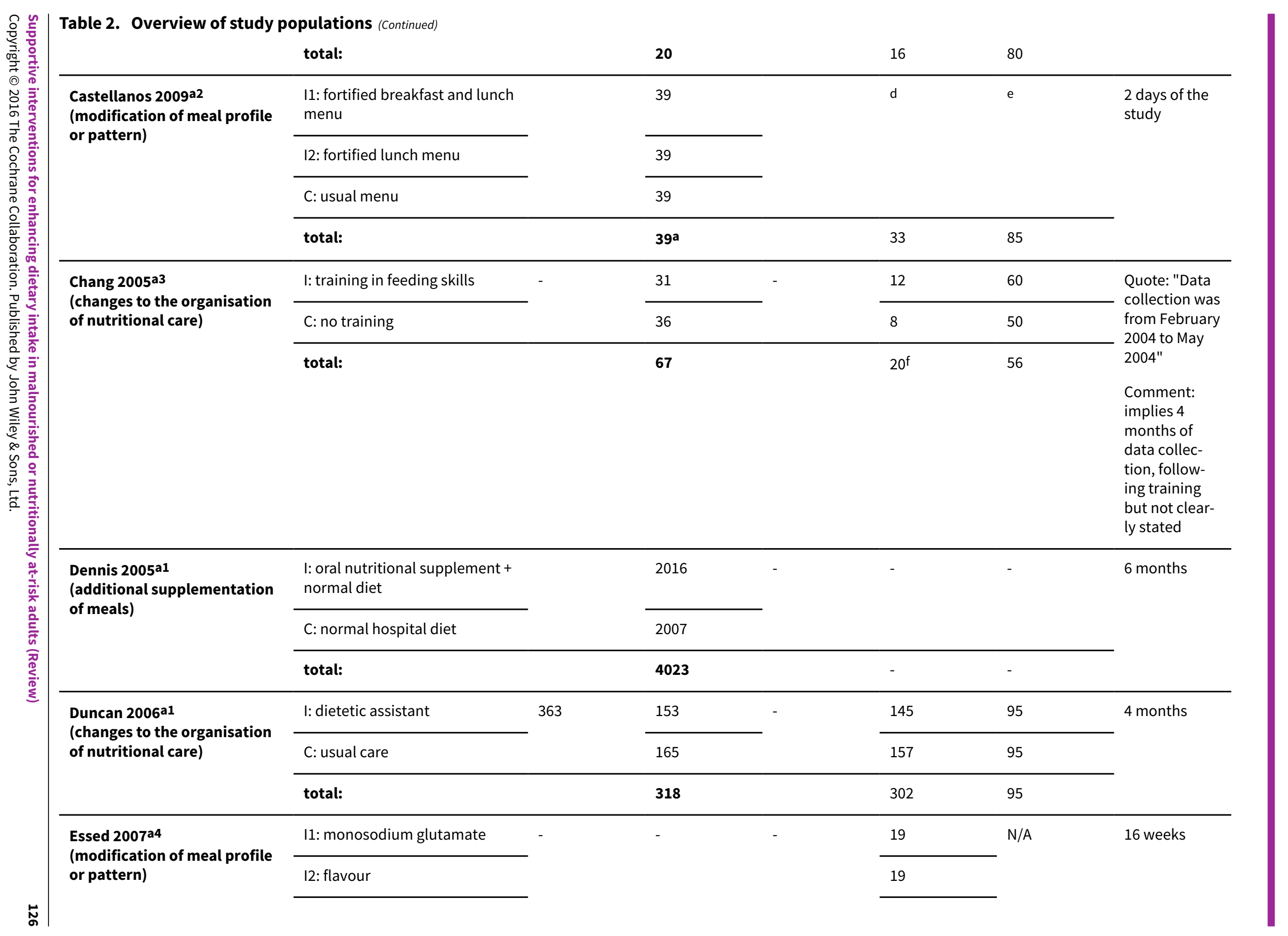




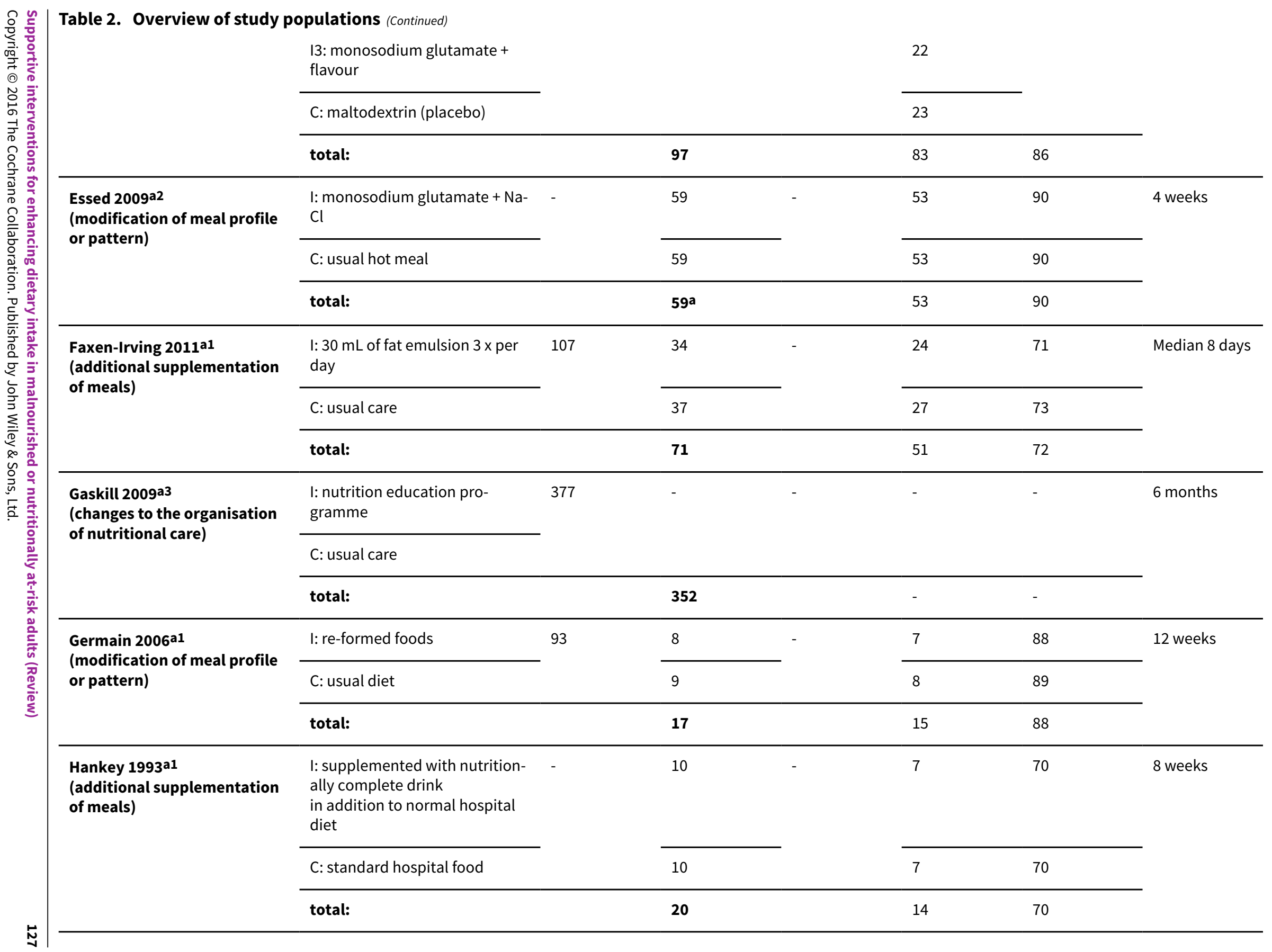

Essed 2009a2

I: monosodium glutamate $+\mathrm{Na}$ 


\begin{tabular}{|c|c|c|c|c|c|c|c|}
\hline \multirow{3}{*}{$\begin{array}{l}\text { Hickson } 2004^{a 1} \\
\text { (changes to the organisation } \\
\text { of nutritional care) }\end{array}$} & I: feeding assistance & 1776 & 292 & 292 & 250 & 86 & \multirow{3}{*}{$\begin{array}{l}\text { Duration of } \\
\text { hospital stay }\end{array}$} \\
\hline & C: usual care & & 300 & 300 & 259 & 86 & \\
\hline & total: & & 592 & 592 & 509 & 86 & \\
\hline \multirow{3}{*}{$\begin{array}{l}\text { Holyday } 2012^{\mathrm{a} 1} \\
\text { (changes to the organisation } \\
\text { of nutritional care) }\end{array}$} & I: malnutrition care plan & - & 71 & 71 & 71 & 100 & \multirow{3}{*}{$\begin{array}{l}\text { Duration of } \\
\text { hospital stay }\end{array}$} \\
\hline & C: usual care & & 72 & 72 & 72 & 100 & \\
\hline & total: & & 143 & 143 & 143 & 100 & \\
\hline \multirow{3}{*}{$\begin{array}{l}\text { Johansen } 2004^{a 1} \\
\text { (changes to the organisation } \\
\text { of nutritional care) }\end{array}$} & I: nutrition team & 7468 & - & - & 108 & $\mathrm{~N} / \mathrm{A}$ & \multirow{3}{*}{$\begin{array}{l}\text { Duration of } \\
\text { hospital stay }\end{array}$} \\
\hline & C: usual care & & & & 104 & & \\
\hline & total: & & 215 & & 212 & 99 & \\
\hline \multirow{3}{*}{$\begin{array}{l}\text { Kraft } 2012^{\mathrm{a} 1} \\
\text { (changes to the organisation } \\
\text { of nutritional care) }\end{array}$} & $\begin{array}{l}\text { I: oral nutritional supplement + } \\
\text { telemedicine monitoring }\end{array}$ & $87 / 50$ & 13 & 5 & 1 & 8 & \multirow[t]{3}{*}{6 months } \\
\hline & C: usual care & & 13 & 9 & 4 & 31 & \\
\hline & total: & & 26 & 14 & 5 & 19 & \\
\hline \multirow{3}{*}{$\begin{array}{l}\text { Kretser } 2003^{a 1} \\
\text { (congregate and home meal } \\
\text { delivery systems) }\end{array}$} & I: modified meals on wheels & 324 & 102 & - & - & - & \multirow[t]{3}{*}{26 weeks } \\
\hline & C: traditional meals on wheels & & 101 & & & & \\
\hline & total: & & 203 & & 60 & 30 & \\
\hline \multirow{3}{*}{$\begin{array}{l}\text { Larsson } 1990^{a 1} \\
\text { (additional supplementation } \\
\text { of meals) }\end{array}$} & $\begin{array}{l}\text { I: oral nutritional supplement + } \\
\text { normal hospital diet }\end{array}$ & - & 197 & - & - & - & \multirow[t]{3}{*}{26 weeks } \\
\hline & C: normal hospital diet & & 238 & & & & \\
\hline & total: & & 435 & & - & - & \\
\hline \multirow{2}{*}{$\begin{array}{l}\text { Leslie } 2012^{\mathrm{a} 3} \\
\text { (modification of meal profile } \\
\text { or pattern) }\end{array}$} & I: energy enriched usual meals & 445 & 22 & & 16 & 73 & \multirow[t]{2}{*}{12 weeks } \\
\hline & C: usual care & & 19 & & 16 & 84 & \\
\hline
\end{tabular}




\begin{tabular}{|c|c|c|c|c|c|c|c|}
\hline & total: & & 41 & & & & \\
\hline \multirow{4}{*}{$\begin{array}{l}\text { Lin } 2010^{\mathrm{a} 3} \\
\text { (changes to the organisation } \\
\text { of nutritional care) }\end{array}$} & I1: spaced-retrievalg & - & 32 & - & - & - & 8 weeks \\
\hline & 12: Montessorih & & 29 & & & & \\
\hline & C: usual care & & 24 & & & & \\
\hline & total: & & 85 & & 82 & 97 & \\
\hline \multirow{3}{*}{$\begin{array}{l}\text { Lin } 2011^{\mathrm{a} 2} \text {, a3 } \\
\text { (changes to the organisation } \\
\text { of nutritional care) }\end{array}$} & I: Montessori & & - & - & - & - & 8 weeks \\
\hline & C: usual care & & & & & & \\
\hline & total: & & $29 a$ & & 29 & 100 & \\
\hline \multirow{3}{*}{$\begin{array}{l}\text { Mathey } 2001 a^{a 3} \\
\text { (changes to the feeding envi- } \\
\text { ronment) }\end{array}$} & I: improved meal ambiance & 60 & 21 & - & 12 & 57 & 12 months \\
\hline & C: usual care & & 17 & & 10 & 59 & \\
\hline & total: & & 38 & & 22 & 58 & \\
\hline \multirow{3}{*}{$\begin{array}{l}\text { Mathey } 2001 b^{a 1} \\
\text { (changes to the feeding envi- } \\
\text { ronment) }\end{array}$} & I: flavour enhancement & - & - & - & 31 & $\mathrm{~N} / \mathrm{A}$ & 16 weeks \\
\hline & C: usual care & & & & 36 & & \\
\hline & total: & & 71 & & 67 & 94 & \\
\hline \multirow[t]{3}{*}{$\begin{array}{l}\text { Munk } 2014 \text { a1 } \\
\text { (modification of meal profile } \\
\text { or pattern) }\end{array}$} & $\begin{array}{l}\text { I: energy and protein enriched } \\
\text { foods provided via a la carte } \\
\text { menu in addition to hospital } \\
\text { food }\end{array}$ & & 44 & & 41 & 96 & $\begin{array}{l}\text { Duration of } \\
\text { hospital stay }\end{array}$ \\
\hline & C: usual care & & 40 & & 40 & & \\
\hline & total: & & 84 & & & & \\
\hline \multirow{3}{*}{$\begin{array}{l}\text { Nijs } 2006 \text { a3 } \\
\text { (changes to the feeding envi- } \\
\text { ronment) }\end{array}$} & I: family-style meals & 282 & 133 & - & 95 & 71 & 6 months \\
\hline & C: usual care & & 112 & & 83 & 74 & \\
\hline & total: & & 245 & & 178 & 73 & \\
\hline
\end{tabular}




\begin{tabular}{|c|c|c|c|c|c|c|c|}
\hline \multirow{3}{*}{$\begin{array}{l}\text { Olofsson } 2007^{a 1} \\
\text { (changes to the organisation } \\
\text { of nutritional care) }\end{array}$} & $\begin{array}{l}\text { I: multi-component interven- } \\
\text { tion (including nutrition) }\end{array}$ & 353 & 102 & - & 83 & 81 & \multirow[t]{3}{*}{4 months } \\
\hline & C: usual care & & 97 & & 74 & 76 & \\
\hline & total: & & 199 & & 157 & 79 & \\
\hline \multirow{4}{*}{$\begin{array}{l}\text { Pivi } 2011^{\text {a1 }} \\
\text { (changes to the organisation } \\
\text { of nutritional care) }\end{array}$} & I1: nutrition education & - & - & - & 25 & $\mathrm{~N} / \mathrm{A}$ & 6 months \\
\hline & 12: oral nutritional supplements & & & & 26 & & \\
\hline & C: usual care & & & & 27 & & \\
\hline & total: & & 90 & & 78 & 87 & \\
\hline \multirow{3}{*}{$\begin{array}{l}\text { Potter } 2001^{\mathrm{a} 1} \\
\text { (additional supplementation } \\
\text { of meals) }\end{array}$} & $\begin{array}{l}\text { I: oral nutritional supplement + } \\
\text { normal hospital diet }\end{array}$ & 618 & 186 & - & 186 & 100 & $\begin{array}{l}\text { Duration of } \\
\text { hospital stay }\end{array}$ \\
\hline & C: normal hospital diet & & 195 & & 195 & 100 & \\
\hline & total: & & 381 & & 381 & 100 & \\
\hline \multirow{3}{*}{$\begin{array}{l}\text { Remsburg } 2001^{\mathrm{a} 1} \\
\text { (changes to the feeding envi- } \\
\text { ronment) }\end{array}$} & I: buffet-style meals & 62 & 20 & - & 20 & 100 & 3 months \\
\hline & C: usual care & & 20 & & 19 & 95 & \\
\hline & total: & & 40 & & 39 & 98 & \\
\hline \multirow{3}{*}{$\begin{array}{l}\text { Salva } 2011^{\mathrm{a} 3} \\
\text { (changes to the organisation } \\
\text { of nutritional care) }\end{array}$} & I: teaching and training & - & 448 & 448 & 300 & 67 & 12 months \\
\hline & C: usual care & & 498 & 498 & 368 & 74 & \\
\hline & total: & & 946 & 946 & 668 & 71 & \\
\hline \multirow{3}{*}{$\begin{array}{l}\text { Silver } 2008^{\mathrm{a} 2} \\
\text { (modification of meal profile } \\
\text { or pattern) }\end{array}$} & $\begin{array}{l}\text { I: fortified home-delivered } \\
\text { lunch }\end{array}$ & - & - & - & - & - & 7 months \\
\hline & C: usual home-delivered lunch & & & & & & \\
\hline & total: & & 52 & & 45 & 87 & \\
\hline
\end{tabular}




\begin{tabular}{|c|c|c|c|c|c|c|c|}
\hline \multirow{3}{*}{$\begin{array}{l}\text { Simmons } 2008 \mathrm{a} 2 \text {, a3 } \\
\text { (additional supplementation } \\
\text { of meals) }\end{array}$} & $\begin{array}{l}\text { I: feeding assistance and/or } \\
\text { snacks }\end{array}$ & 173 & 30 & - & 28 & 88 & \multirow[t]{3}{*}{24 weeks } \\
\hline & C: usual diet & & 34 & & 32 & 94 & \\
\hline & total: & & $64^{a}$ & - & 60 & 94 & \\
\hline \multirow{4}{*}{$\begin{array}{l}\text { Simmons } 2010^{\mathrm{a} 1} \\
\text { (additional supplementation } \\
\text { of meals) }\end{array}$} & I1: snacks & 280 & - & - & 25 & $\mathrm{~N} / \mathrm{A}$ & \multirow[t]{4}{*}{6 weeks } \\
\hline & $\begin{array}{l}\text { I2: additional supplementation } \\
\text { of meals }\end{array}$ & & & & 18 & & \\
\hline & C: usual care & & & & 20 & & \\
\hline & total: & & 86 & & 63 & 73 & \\
\hline \multirow{3}{*}{$\begin{array}{l}\text { Smolliner } 2008^{\mathrm{a} 3} \\
\text { (modification of meal profile } \\
\text { or pattern) }\end{array}$} & I: fortified meals and snacks & $295 / 92$ & - & - & 22 & $\mathrm{~N} / \mathrm{A}$ & \multirow[t]{3}{*}{12 weeks } \\
\hline & C: usual diet & & & & 30 & & \\
\hline & total: & & 65 & & 52 & 80 & \\
\hline \multirow{3}{*}{$\begin{array}{l}\text { Splett } 2003^{a 3} \\
\text { (changes to the organisation } \\
\text { of nutritional care) }\end{array}$} & I: medical nutrition therapy & 394 & 223 & - & 200 & 90 & \multirow[t]{3}{*}{$19-180$ days } \\
\hline & C: usual care & & 171 & & 164 & 96 & \\
\hline & total: & & 394 & & 364 & 92 & \\
\hline \multirow{3}{*}{$\begin{array}{l}\text { Taylor } 2006^{a 2} \\
\text { (modification of meal profile } \\
\text { or pattern) }\end{array}$} & I: 5-meal menu & 66 & - & - & - & - & \multirow{3}{*}{$\begin{array}{l}2 \text { periods of } 4 \\
\text { days }\end{array}$} \\
\hline & C: usual (3-meal menu) & & & & & & \\
\hline & total: & & $31 a$ & & 31 & 100 & \\
\hline \multirow{3}{*}{$\begin{array}{l}\text { Van den Berg 2015a1 } \\
\text { (additional supplementation } \\
\text { of meals }\end{array}$} & $\begin{array}{l}\text { I1: offered } 125 \mathrm{~mL} \text { ONS daily } \\
\text { with medication rounds }\end{array}$ & 885 & 88 & & 75 & 85 & \multirow[t]{3}{*}{$\begin{array}{l}\text { Maximum pe- } \\
\text { riod } 30 \text { days }\end{array}$} \\
\hline & $\begin{array}{l}\text { 12: offered } 62 \mathrm{~mL} \text { ONS daily with } \\
\text { medication rounds }\end{array}$ & & 66 & & 51 & 77 & \\
\hline & $\begin{array}{l}\text { C: offered } 125 \mathrm{~mL} \text { ONS twice } \\
\text { daily in between meals }\end{array}$ & & 80 & & 66 & 83 & \\
\hline
\end{tabular}




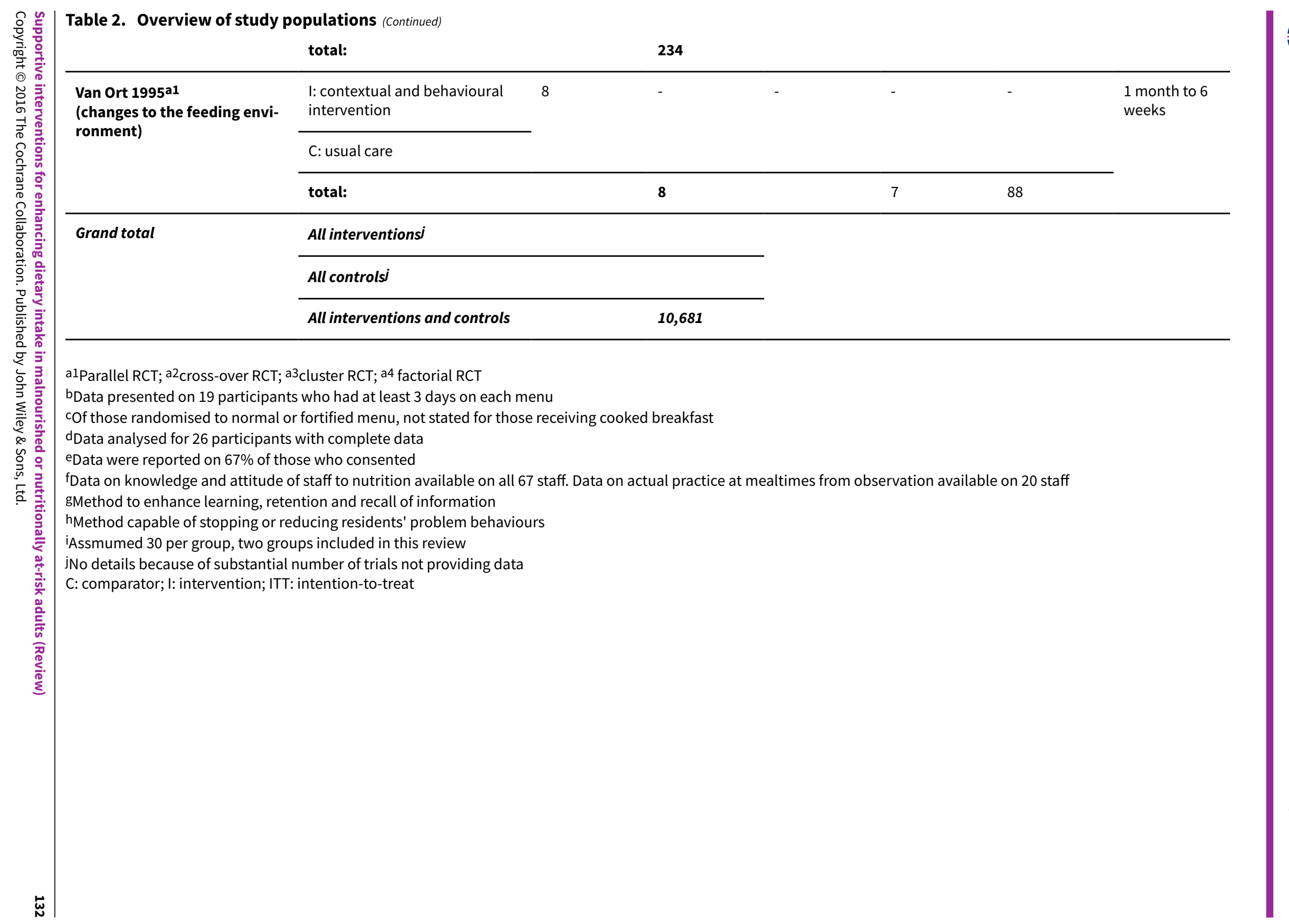


Table 3. Summary of outcomes reported in intervention category 1: changes to the organisation of nutritional care

\begin{tabular}{|c|c|c|c|}
\hline Outcome measure & $\begin{array}{l}\text { No. of studies } \\
\text { reporting out- } \\
\text { come }\end{array}$ & No. of participants & $\begin{array}{l}\text { Studies potential- } \\
\text { ly with data for } \\
\text { meta-analysis }\end{array}$ \\
\hline Energy intake & 5 & 666 & 1 \\
\hline Health-related quality of life & 1 & 220 & 0 \\
\hline Patient satisfaction & 2 & 1105 & 0 \\
\hline Complications & 4 & 1263 & 3 \\
\hline Nutritional status: weight & 10 & 2184 & 9 \\
\hline BMI & 7 & 1537 & 6 \\
\hline TSF & 3 & 536 & 3 \\
\hline MAC & 3 & 568 & 3 \\
\hline Length of stay & 5 & 1256 & 3 \\
\hline Hospital admission & 1 & 143 & 1 \\
\hline Mortality & 5 & 2182 & 5 \\
\hline Costs & 2 & 1089 & 0 \\
\hline
\end{tabular}

BMI: body mass index; MAC: mid-arm circumference; TSF: triceps skinfold thickness

Table 4. Summary of outcomes reported in intervention category 2: changes to the feeding environment

\begin{tabular}{llll}
\hline Outcome measure & $\begin{array}{l}\text { No. of studies } \\
\text { reporting out- } \\
\text { come }\end{array}$ & $\begin{array}{l}\text { No. of participants } \\
\text { (treatment/control) }\end{array}$ & $\begin{array}{l}\text { Studies with data } \\
\text { for meta-analysis }\end{array}$ \\
\hline Energy intake & 3 & 216 & 3 \\
\hline Health-related quality of life & 2 & 200 & 0 \\
\hline Nutritional status: weight & 3 & 239 & 3 \\
\hline MAC & 1 & 178 & 1 \\
\hline Clinical function & 3 & 1664 & 2 \\
\hline Mortality & 3 & 236 & 3 \\
\hline
\end{tabular}

MAC: mid-arm circumference 
Table 5. Summary of outcomes reported in intervention category 3: modification of meal profile or pattern

\begin{tabular}{llll}
\hline Outcome measure & $\begin{array}{l}\text { No. of studies } \\
\text { reporting out- } \\
\text { come }\end{array}$ & No. of participants & $\begin{array}{l}\text { Studies potentially } \\
\text { with data for meta- } \\
\text { analysis }\end{array}$ \\
\hline Energy intake & 11 & 506 & 7 \\
\hline Health-related quality of life & 1 & 52 & 0 \\
\hline Complications & 1 & 66 & 1 \\
\hline Nutritional status: weight & 7 & 387 & 3 \\
\hline BMI & 3 & 98 & 1 \\
\hline MAC & 1 & 32 & 3 \\
\hline Clinical function & 3 & 200 & 1 \\
\hline Length of stay & 1 & 81 & 4 \\
\hline Mortality & 4 & 243 & 3 \\
\hline
\end{tabular}

BMI: body mass index; MAC: mid-arm circumference

Table 6. Summary of outcomes reported in intervention category 4: additional supplementation of meals

\begin{tabular}{llll} 
Outcome measure & $\begin{array}{l}\text { No. of studies } \\
\text { reporting out- } \\
\text { come }\end{array}$ & No. of participants & $\begin{array}{l}\text { Studies potentially } \\
\text { with data for meta- } \\
\text { analysis }\end{array}$ \\
\hline Energy intake & 8 & 1469 & 7 \\
\hline Health-related quality of life & 1 & 4023 & 0 \\
\hline Complications & 2 & 4695 & 1 \\
\hline Nutritional status: weight & 7 & 605 & 4 \\
\hline BMI & 2 & 102 & 1 \\
\hline TSF & 2 & & 0 \\
\hline MAC & 3 & 1 \\
\hline Clinical function & 2 & 618 & 0 \\
\hline Length of stay & 4 & 4689 & 1 \\
\hline Mortality & 5 & 5745 & 5 \\
\hline Costs & 1 & 63 & 0 \\
\hline
\end{tabular}

BMI: body mass index; MAC: mid-arm circumference; TSF: triceps skinfold thickness 
Table 7. Summary of outcomes reported in all interventions

\begin{tabular}{|c|c|c|c|}
\hline Outcome measure & $\begin{array}{l}\text { No. of studies } \\
\text { reporting out- } \\
\text { come }\end{array}$ & $\begin{array}{l}\text { No. of participants } \\
\text { (treatment/control) }\end{array}$ & $\begin{array}{l}\text { Studies included } \\
\text { in the meta-analy- } \\
\text { sis }\end{array}$ \\
\hline Energy intake & 27 & 2857 & 0 \\
\hline Health-related quality of life & 5 & 4495 & 0 \\
\hline Patient satisfaction & 2 & 1105 & 0 \\
\hline Complications & 7 & 6024 & 5 \\
\hline Nutritional status: weight & 28 & 3618 & 24 \\
\hline BMI & 12 & 1737 & 0 \\
\hline TSF & 5 & - & 0 \\
\hline MAC & 8 & - & 0 \\
\hline Clinical function & 9 & 2746 & 0 \\
\hline Length of hospital stay & 10 & 6026 & 5 \\
\hline Hospital admissions & 2 & 389 & 0 \\
\hline Mortality & 18 & 8690 & 17 \\
\hline Economic costs & 3 & 1152 & 0 \\
\hline
\end{tabular}

BMI: body mass index; MAC: mid-arm circumference; TSF: triceps skinfold thickness

Table 8. Reasons for contacting authors, and outcomes of contact with authors

\begin{tabular}{|c|c|c|c|c|c|}
\hline & Outcome & $\begin{array}{l}\text { Reason the data were not us- } \\
\text { able }\end{array}$ & $\begin{array}{l}\text { Contact with au- } \\
\text { thor }\end{array}$ & $\begin{array}{l}\text { Outcome of } \\
\text { contact with } \\
\text { author }\end{array}$ & Action taken \\
\hline \multicolumn{6}{|c|}{ 1. Organisational change } \\
\hline Chang 2005 & Energy intake & $\begin{array}{l}\text { Data reported as amount eat- } \\
\text { en in } 1 / 4,1 / 2,3 / 4\end{array}$ & Yes & No response & $\begin{array}{l}\text { Data reported in } \\
\text { structured narrative } \\
\text { summary }\end{array}$ \\
\hline \multirow[t]{2}{*}{ Duncan 2006} & Complications & Reported as a median and IQR & Yes & Data provided & Data used \\
\hline & Length of stay & Reported as median and IQR & Yes & $\begin{array}{l}\text { Confirmed data } \\
\text { skewed }\end{array}$ & $\begin{array}{l}\text { Data reported in } \\
\text { structured narrative } \\
\text { summary }\end{array}$ \\
\hline Gaskill 2009 & $\begin{array}{l}\text { Measured } \\
\text { prevalence of } \\
\text { malnutrition } \\
\text { with SGA }\end{array}$ & $\begin{array}{l}\text { Not an outcome of interest for } \\
\text { this review }\end{array}$ & $\begin{array}{l}\text { Yes, to request } \\
\text { weight data (a com- } \\
\text { ponent of SGA) }\end{array}$ & $\begin{array}{l}\text { Unable to pro- } \\
\text { vide data }\end{array}$ & Data not reported \\
\hline
\end{tabular}


Table 8. Reasons for contacting authors, and outcomes of contact with authors

\begin{tabular}{|c|c|c|c|c|c|}
\hline \multirow[t]{3}{*}{ Hickson 2004} & Energy intake & $\begin{array}{l}\text { Not measured at baseline, on- } \\
\text { ly at follow-up }\end{array}$ & $\begin{array}{l}\text { Yes, to confirm in- } \\
\text { terpretation of data }\end{array}$ & $\begin{array}{l}\text { Data not mea- } \\
\text { sured at base- } \\
\text { line }\end{array}$ & $\begin{array}{l}\text { Data reported in } \\
\text { structured narrative } \\
\text { summary }\end{array}$ \\
\hline & $\begin{array}{l}\text { Complications } \\
\text { (antibiotic } \\
\text { prescription) }\end{array}$ & Reported as median and IQR & $\begin{array}{l}\text { Yes, to request } \\
\text { complications ac- } \\
\text { cording to group al- } \\
\text { location }\end{array}$ & $\begin{array}{l}\text { No. complica- } \\
\text { tions according } \\
\text { to group allo- } \\
\text { cation was pro- } \\
\text { vided }\end{array}$ & $\begin{array}{l}\text { Data reported in } \\
\text { structured narrative } \\
\text { summary }\end{array}$ \\
\hline & $\begin{array}{l}\text { Hospital ad- } \\
\text { mission }\end{array}$ & $\begin{array}{l}\text { States in protocol these are } \\
\text { collected, but not reported }\end{array}$ & Yes, to request data & $\begin{array}{l}\text { Author unable } \\
\text { to recall what } \\
\text { happened with } \\
\text { data }\end{array}$ & Data not reported \\
\hline
\end{tabular}

\begin{tabular}{|c|c|c|c|c|c|}
\hline Holyday 2012 & Costs & $\begin{array}{l}\text { An estimate based on local } \\
\text { prices, not a complete cost } \\
\text { analysis }\end{array}$ & $\begin{array}{l}\text { No, judged unlikely } \\
\text { to be available }\end{array}$ & $\mathrm{N} / \mathrm{A}$ & Data not reported \\
\hline & $\begin{array}{l}\text { Hospital ad- } \\
\text { mission }\end{array}$ & Presented as a frequency & $\begin{array}{l}\text { Yes, to request total } \\
\text { number of readmis- } \\
\text { sions }\end{array}$ & Data provided & $\begin{array}{l}\text { Data reported in } \\
\text { structured narrative } \\
\text { summary }\end{array}$ \\
\hline $\begin{array}{l}\text { Johansen } \\
2004\end{array}$ & Energy intake & Reported as kJ/kg/day & $\begin{array}{l}\text { Yes, for mean } \\
\text { change }\end{array}$ & No response & Data not reported \\
\hline Kraft 2012 & BMI & $\begin{array}{l}\text { Presented as mean and SD at } \\
\text { baseline and follow-up, but no } \\
\text { mean change }\end{array}$ & Yes & No response & Data not reported \\
\hline \multirow[t]{3}{*}{ Lin 2010} & Energy intake & $\begin{array}{l}\text { 'Amount of each meal con- } \\
\text { sumed' was reported as \% eat- } \\
\text { en }\end{array}$ & Yes & No response & $\begin{array}{l}\text { Data reported in } \\
\text { structured narrative } \\
\text { summary }\end{array}$ \\
\hline & Weight & $\begin{array}{l}\text { Reported as mean and SD pre } \\
\text { and post intervention/control }\end{array}$ & $\begin{array}{l}\text { Yes, to request } \\
\text { mean change }\end{array}$ & No response & $\begin{array}{l}\text { Calculated mean } \\
\text { change, and imput- } \\
\text { ed the SD of change } \\
\text { from Salva } 2011\end{array}$ \\
\hline & BMI & $\begin{array}{l}\text { Reported as mean and SD pre } \\
\text { and post intervention/control }\end{array}$ & $\begin{array}{l}\text { Yes, to request } \\
\text { mean change }\end{array}$ & No response & $\begin{array}{l}\text { Calculated mean } \\
\text { change, and imput- } \\
\text { ed the SD of change } \\
\text { from Salva } 2011\end{array}$ \\
\hline \multirow[t]{3}{*}{ Olofsson 2007} & Weight & $\begin{array}{l}\text { Reported as mean and SD pre } \\
\text { and post intervention/control }\end{array}$ & $\begin{array}{l}\text { Yes, to request } \\
\text { mean change and } \\
\text { SD }\end{array}$ & Data provided & $\begin{array}{l}\text { Data reported in } \\
\text { structured narrative } \\
\text { summary }\end{array}$ \\
\hline & BMI & $\begin{array}{l}\text { Reported as mean and SD pre } \\
\text { and post intervention/control }\end{array}$ & $\begin{array}{l}\text { Yes, to request } \\
\text { mean change and } \\
\text { SD }\end{array}$ & Data provided & $\begin{array}{l}\text { Data reported in } \\
\text { structured narrative } \\
\text { summary }\end{array}$ \\
\hline & Complications & $\begin{array}{l}\text { Reported as no. falls in men } \\
\text { and women }\end{array}$ & $\begin{array}{l}\text { Yes, to request total } \\
\text { complications per } \\
\text { group }\end{array}$ & Data provided & $\begin{array}{l}\text { Data reported in } \\
\text { structured narrative } \\
\text { summary }\end{array}$ \\
\hline Pivi 2011 & Weight & $\begin{array}{l}\text { Reported as mean and SD pre } \\
\text { and post intervention/control }\end{array}$ & $\begin{array}{l}\text { Yes, to request } \\
\text { mean change }\end{array}$ & No response & $\begin{array}{l}\text { Calculated mean } \\
\text { change, and imputed }\end{array}$ \\
\hline
\end{tabular}


Table 8. Reasons for contacting authors, and outcomes of contact with authors

(Continued)

the SD of change us-

ing the $P$ value

\begin{tabular}{lll}
\hline BMI & $\begin{array}{l}\text { Reported as mean and SD pre } \\
\text { and post intervention/control }\end{array}$ & $\begin{array}{l}\text { Yes, to request } \\
\text { mean change }\end{array}$
\end{tabular}$\quad$ No response

Calculated mean change, and imputed the SD of change from Salva 2011

$\begin{array}{lll}\text { TSF } & \begin{array}{l}\text { Reported as mean and SD pre } \\ \text { and post intervention/control }\end{array} & \begin{array}{l}\text { Yes, to request } \\ \text { mean change }\end{array}\end{array} \quad$ No response

Calculated mean change, and imputed the SD of change from Salva 2011

\begin{tabular}{|c|c|c|c|c|}
\hline MAC & $\begin{array}{l}\text { Reported as mean and SD pre } \\
\text { and post intervention/control }\end{array}$ & $\begin{array}{l}\text { Yes, to request } \\
\text { mean change }\end{array}$ & No response & $\begin{array}{l}\text { Calculated mean } \\
\text { change, and imputed } \\
\text { the SD of change }\end{array}$ \\
\hline
\end{tabular}

\begin{tabular}{|c|c|c|c|c|c|}
\hline \multirow[t]{2}{*}{ Salva 2011} & MAC & $\begin{array}{l}\text { Methodology reported this } \\
\text { was an outcome measured, } \\
\text { but not reported in results }\end{array}$ & Yes & No response & Data not used \\
\hline & Costs & $\begin{array}{l}\text { Described as data to be col- } \\
\text { lected, but reported that } \\
\text { analysis was not undertaken }\end{array}$ & No & & Not reported \\
\hline \multirow[t]{2}{*}{ Splett 2003} & Intake & $\begin{array}{l}\text { Food intake is documented as } \\
\text { a nutrition assessment activity }\end{array}$ & $\begin{array}{l}\text { Yes, to request } \\
\text { mean energy intake } \\
\text { per group }\end{array}$ & $\begin{array}{l}\text { Unable to pro- } \\
\text { vide data }\end{array}$ & Not reported \\
\hline & Weight & $\begin{array}{l}\text { Methodology reports this was } \\
\text { an outcome measured, but re- } \\
\text { ported in a format not usable }\end{array}$ & Yes & $\begin{array}{l}\text { Unable to pro- } \\
\text { vide data }\end{array}$ & Not reported \\
\hline
\end{tabular}

\section{Feeding environment}

\begin{tabular}{llllll}
\hline $\begin{array}{l}\text { Brouilette } \\
1991\end{array}$ & Energy & $\begin{array}{l}\text { Reported pre and post inter- } \\
\text { vention data, but no SD of } \\
\text { change }\end{array}$ & $\begin{array}{l}\text { No, as no author } \\
\text { contact details and } \\
\text { study published in } \\
1991\end{array}$ & N/A & $\begin{array}{l}\text { Imputed the SD from } \\
\text { Nijs 2006 }\end{array}$ \\
\hline Van Ort 1995 & $\begin{array}{l}\text { Weight } \\
\text { change }\end{array}$ & No figures reported & $\begin{array}{l}\text { Yes, to request da- } \\
\text { ta on mean and SD } \\
\text { of change for each } \\
\text { group }\end{array}$ & $\begin{array}{l}\text { Waiting re- } \\
\text { sponse }\end{array}$ & Not used \\
& $\begin{array}{llll}\text { Intervention } \\
\text { group clarifi- } \\
\text { cation }\end{array}$ & $\begin{array}{l}\text { Were the behavioural and con- } \\
\text { textual intervention received } \\
\text { at the same time }\end{array}$ & $\begin{array}{l}\text { Yes, to request this } \\
\text { detail }\end{array}$ & $\begin{array}{l}\text { Waiting re- } \\
\text { sponse }\end{array}$ & $\begin{array}{l}\text { Assumed the two in- } \\
\text { terventions were giv- } \\
\text { en at the same time }\end{array}$
\end{tabular}

\section{Meal modification}

\begin{tabular}{|c|c|c|c|c|c|}
\hline $\begin{array}{l}\text { Bouillanne } \\
2013\end{array}$ & Weight & $\begin{array}{l}\text { Did not report weight, but as- } \\
\text { sumed they had the data as } \\
\text { Full Body Composition was } \\
\text { used }\end{array}$ & Yes, to request data & Data provided & Data reported \\
\hline & Energy intake & Reported as kcal/kg/day & Yes, to request data & Data provided & Data reported \\
\hline
\end{tabular}


Table 8. Reasons for contacting authors, and outcomes of contact with authors

\begin{tabular}{|c|c|c|c|c|c|}
\hline & $\begin{array}{l}\text { Hand grip } \\
\text { strength }\end{array}$ & $\begin{array}{l}\text { Reported data as mean/medi- } \\
\text { an and } 95 \% \mathrm{Cl} \text { of the median }\end{array}$ & Yes, to request data & $\begin{array}{l}\text { Provided mean } \\
\text { and SD of } \\
\text { change }\end{array}$ & Data reported \\
\hline & $A D L$ & $\begin{array}{l}\text { Reported data as mean/medi- } \\
\text { an and } 95 \% \mathrm{Cl} \text { of the median }\end{array}$ & Yes, to request data & Data provided & Data reported \\
\hline $\begin{array}{l}\text { Castellanos } \\
2009\end{array}$ & Energy intake & $\begin{array}{l}\text { Results were not analysed ac- } \\
\text { cording to groups randomised, } \\
\text { but regrouped subjects into } \\
\text { small eaters and large eaters }\end{array}$ & $\begin{array}{l}\text { Yes, to ask for da- } \\
\text { ta on mean and SD } \\
\text { of change for each } \\
\text { group }\end{array}$ & No response & Data reported \\
\hline Germain 2006 & BMI & $\begin{array}{l}\text { They reported the mean BMI } \\
\text { rather than mean change }\end{array}$ & $\begin{array}{l}\text { Yes, for mean and } \\
\text { SD of change }\end{array}$ & Data provided & Data reported \\
\hline \multirow[t]{4}{*}{$\begin{array}{l}\text { Smolliner } \\
2008\end{array}$} & $\begin{array}{l}\text { Weight } \\
\text { change }\end{array}$ & $\begin{array}{l}\text { Reported mean and SD at } \\
\text { baseline and end of interven- } \\
\text { tion }\end{array}$ & $\begin{array}{l}\text { Yes, for mean } \\
\text { change and SD }\end{array}$ & Data provided & Data reported \\
\hline & BMI & $\begin{array}{l}\text { Reported mean and SD at } \\
\text { baseline and end of interven- } \\
\text { tion }\end{array}$ & $\begin{array}{l}\text { Yes, for mean } \\
\text { change and SD }\end{array}$ & Data provided & Data reported \\
\hline & $\begin{array}{l}\text { Handgrip } \\
\text { strength }\end{array}$ & $\begin{array}{l}\text { Reported mean and SD at } \\
\text { baseline and end of interven- } \\
\text { tion }\end{array}$ & $\begin{array}{l}\text { Yes, for mean } \\
\text { change and SD }\end{array}$ & Data provided & Data reported \\
\hline & $\begin{array}{l}\text { health-related } \\
\text { quality of life }\end{array}$ & $\begin{array}{l}\text { Reported mean and SD at } \\
\text { baseline and end of interven- } \\
\text { tion }\end{array}$ & $\begin{array}{l}\text { Yes, for mean } \\
\text { change and SD }\end{array}$ & Data provided & Data reported \\
\hline
\end{tabular}

\section{Supplementation of meals}

\begin{tabular}{|c|c|c|c|c|c|}
\hline Beck 2002 & Weight & $\begin{array}{l}\text { Reported as median change } \\
\text { with } 95 \% \mathrm{Cl}\end{array}$ & $\begin{array}{l}\text { Yes, for mean } \\
\text { change and SD }\end{array}$ & $\begin{array}{l}\text { Response re- } \\
\text { ceived but data } \\
\text { not available }\end{array}$ & $\begin{array}{l}\text { Data reported in } \\
\text { structured narrative } \\
\text { summary }\end{array}$ \\
\hline & Energy intake & $\begin{array}{l}\text { Reported as median change } \\
\text { with } 95 \% \mathrm{Cl}\end{array}$ & $\begin{array}{l}\text { Yes, for mean } \\
\text { change and SD }\end{array}$ & $\begin{array}{l}\text { Response re- } \\
\text { ceived but data } \\
\text { not available }\end{array}$ & $\begin{array}{l}\text { Data reported in } \\
\text { structured narrative } \\
\text { summary }\end{array}$ \\
\hline \multirow[t]{2}{*}{$\begin{array}{l}\text { Bourdel- Mar- } \\
\text { chasson } 2000\end{array}$} & $\begin{array}{l}\text { Pressure ul- } \\
\text { cers }\end{array}$ & $\begin{array}{l}\text { Data given as percentage per } \\
\text { group }\end{array}$ & $\begin{array}{l}\text { Yes, for number per } \\
\text { group }\end{array}$ & Data provided & $\begin{array}{l}\text { Data reported in } \\
\text { structured narrative } \\
\text { summary }\end{array}$ \\
\hline & Weight & Data given for baseline only & $\begin{array}{l}\text { Yes, for change in } \\
\text { weight from base- } \\
\text { line to follow-up }\end{array}$ & $\begin{array}{l}\text { Yes, author } \\
\text { stated she did } \\
\text { not find the } \\
\text { analysis of dis- } \\
\text { charge weight, } \\
\text { probably due to } \\
\text { the low quality } \\
\text { of this data (too } \\
\text { many missing } \\
\text { data) }\end{array}$ & Data not reported \\
\hline
\end{tabular}


Table 8. Reasons for contacting authors, and outcomes of contact with authors (Continued)

\begin{tabular}{|c|c|c|c|c|c|}
\hline \multirow[t]{2}{*}{ Dennis 2005} & Complications & Data given as percentages & $\begin{array}{l}\text { Yes for data on total } \\
\text { complications per } \\
\text { group }\end{array}$ & Data provided & $\begin{array}{l}\text { Data reported in } \\
\text { structured narrative } \\
\text { summary }\end{array}$ \\
\hline & $\begin{array}{l}\text { Health-related } \\
\text { quality of life } \\
\text { score }\end{array}$ & $\begin{array}{l}\text { Differences between means } \\
\text { provided }\end{array}$ & $\begin{array}{l}\text { Yes, to request } \\
\text { mean and SD of } \\
\text { changes }\end{array}$ & $\begin{array}{l}\text { Unable to pro- } \\
\text { vide data, as } \\
\text { EuroQol was } \\
\text { only measured } \\
\text { at follow-up }\end{array}$ & $\begin{array}{l}\text { Data reported in } \\
\text { structured narrative } \\
\text { summary }\end{array}$ \\
\hline
\end{tabular}

\begin{tabular}{lll}
\hline $\begin{array}{l}\text { Faxen-Irving } \\
2011\end{array}$ & Energy intake & $\begin{array}{l}\text { Data given in a graph, no num- } \\
\text { bers available }\end{array}$ \\
& SD of change in \\
& energy intake, be- \\
& tween the control \\
& and intervention \\
& groups from base- \\
& line to the 2nd reg- \\
& istration
\end{tabular}

\begin{tabular}{|c|c|c|c|c|}
\hline Length of stay & $\begin{array}{l}\text { Data provided at baseline, not } \\
\text { follow-up }\end{array}$ & $\begin{array}{l}\text { Yes, for mean and } \\
\text { SD }\end{array}$ & Data provided & $\begin{array}{l}\text { Data reported in } \\
\text { structured narrative } \\
\text { summary }\end{array}$ \\
\hline
\end{tabular}

\begin{tabular}{|c|c|c|c|c|}
\hline Infection & $\begin{array}{l}\text { Data provided at baseline, not } \\
\text { follow-up }\end{array}$ & $\begin{array}{l}\text { Yes, for mean and } \\
\text { SD }\end{array}$ & $\begin{array}{l}\text { Unable to pro- } \\
\text { vide data }\end{array}$ & Data not reported \\
\hline BMI & $\begin{array}{l}\text { Data provided at baseline, not } \\
\text { follow-up }\end{array}$ & $\begin{array}{l}\text { Yes, for mean and } \\
\text { SD }\end{array}$ & Data provided & $\begin{array}{l}\text { Not reported in the } \\
\text { summary because } \\
\text { few studies mea- } \\
\text { sured this outcome }\end{array}$ \\
\hline
\end{tabular}

\begin{tabular}{lll}
\hline ADL & $\begin{array}{l}\text { Data provided at baseline, not } \\
\text { follow-up }\end{array}$ & $\begin{array}{l}\text { Yes, for mean and } \\
\text { SD }\end{array}$
\end{tabular}$\quad$ Data provided

\section{Not reported in the} summary because few studies measured this outcome

\begin{tabular}{|c|c|c|c|c|c|}
\hline \multirow[t]{3}{*}{ Hankey 1993} & Weight & $\begin{array}{l}\text { Presented in graphs, no num- } \\
\text { bers given }\end{array}$ & $\begin{array}{l}\text { Yes, for mean and } \\
\text { SD }\end{array}$ & $\begin{array}{l}\text { Unable to pro- } \\
\text { vide data but } \\
\text { suggested us- } \\
\text { ing data from } \\
\text { the review by } \\
\text { Milne } 2009 \\
\text { which included } \\
\text { these data }\end{array}$ & $\begin{array}{l}\text { Data obtained from } \\
\text { systematic review by } \\
\text { Milne } 2009\end{array}$ \\
\hline & MAC & $\begin{array}{l}\text { Presented in graphs, no num- } \\
\text { bers given }\end{array}$ & $\begin{array}{l}\text { Yes, for mean and } \\
\text { SD }\end{array}$ & $\begin{array}{l}\text { Unable to pro- } \\
\text { vide data but } \\
\text { suggested us- } \\
\text { ing data from } \\
\text { the review by } \\
\text { Milne } 2009 \\
\text { which included } \\
\text { these data }\end{array}$ & $\begin{array}{l}\text { Data obtained from } \\
\text { systematic review by } \\
\text { Milne } 2009 \text { but not } \\
\text { reported as few stud- } \\
\text { ies measured this } \\
\text { outcome }\end{array}$ \\
\hline & TSF & $\begin{array}{l}\text { Presented in graphs, no num- } \\
\text { bers given }\end{array}$ & $\begin{array}{l}\text { Yes, for mean and } \\
\text { SD }\end{array}$ & $\begin{array}{l}\text { Unable to pro- } \\
\text { vide data but } \\
\text { suggested us- } \\
\text { ing data from } \\
\text { the review by }\end{array}$ & $\begin{array}{l}\text { Not reported in the } \\
\text { summary because } \\
\text { few studies mea- } \\
\text { sured this outcome }\end{array}$ \\
\hline
\end{tabular}


Table 8. Reasons for contacting authors, and outcomes of contact with authors

Milne 2009

which included

these data

\begin{tabular}{|c|c|c|c|c|c|}
\hline & $\begin{array}{l}\text { Energy and } \\
\text { protein intake }\end{array}$ & $\begin{array}{l}\text { Presented in graphs, no num- } \\
\text { bers given }\end{array}$ & $\begin{array}{l}\text { Yes, for mean and } \\
\text { SD }\end{array}$ & $\begin{array}{l}\text { Unable to pro- } \\
\text { vide data }\end{array}$ & Data not reported \\
\hline \multirow[t]{6}{*}{ Larsson 1990} & Energy intake & $\begin{array}{l}\text { Data included in Modified Nor- } \\
\text { ton Scale }\end{array}$ & $\begin{array}{l}\text { Yes, data for change } \\
\text { in energy intake } \\
\text { between groups } \\
\text { (mean and SD) }\end{array}$ & No response & Data not reported \\
\hline & Weight & $\begin{array}{l}\text { Data provided as 'weight in- } \\
\text { dex' }\end{array}$ & $\begin{array}{l}\text { Yes, for change in } \\
\text { weight between } \\
\text { groups (mean and } \\
\text { SD) }\end{array}$ & No response & Data not reported \\
\hline & TSF & $\begin{array}{l}\text { Data provided as differences } \\
\text { between men and women, and } \\
\text { non-PEM and PEM groups }\end{array}$ & $\begin{array}{l}\text { Yes, for change } \\
\text { between groups } \\
\text { (mean and SD) }\end{array}$ & No response & Data not reported \\
\hline & MAC & $\begin{array}{l}\text { Data provided as differences } \\
\text { between men and women, and } \\
\text { non-PEM and PEM groups }\end{array}$ & $\begin{array}{l}\text { Yes, for change } \\
\text { between groups } \\
\text { (mean and SD) }\end{array}$ & No response & Data not reported \\
\hline & Length of stay & Not given & $\begin{array}{l}\text { Yes, for mean and } \\
\text { SD between groups }\end{array}$ & No response & Data not reported \\
\hline & $\begin{array}{l}\text { Total number } \\
\text { of eligible par- } \\
\text { ticipants }\end{array}$ & $\begin{array}{l}\text { Unclear across all } 4 \text { duplicates } \\
\text { of this study }\end{array}$ & $\begin{array}{l}\text { Yes, for a clear num- } \\
\text { ber of randomised } \\
\text { participants, no fin- } \\
\text { ishing study, and } \\
\text { deaths }\end{array}$ & No response & Data not reported \\
\hline \multirow[t]{4}{*}{ Potter 2001} & Length of stay & $\begin{array}{l}\text { Provided as median with a } \\
\text { range }\end{array}$ & $\begin{array}{l}\text { Yes, for mean and } \\
\text { SD between groups }\end{array}$ & No response & $\begin{array}{l}\text { Data reported in } \\
\text { structured narrative } \\
\text { summary }\end{array}$ \\
\hline & $A D L$ & $\begin{array}{l}\text { Stated as an outcome mea- } \\
\text { sure in methodology, then not } \\
\text { reported in results }\end{array}$ & $\begin{array}{l}\text { Yes, for mean and } \\
\text { SD between groups }\end{array}$ & No response & $\begin{array}{l}\text { Not reported in the } \\
\text { summary because } \\
\text { few studies mea- } \\
\text { sured this outcome }\end{array}$ \\
\hline & $\mathrm{BMI}$ & $\begin{array}{l}\text { Stated as an outcome mea- } \\
\text { sure in methodology, then not } \\
\text { reported in results }\end{array}$ & $\begin{array}{l}\text { Yes, for mean and } \\
\text { SD between groups }\end{array}$ & No response & $\begin{array}{l}\text { Not reported in the } \\
\text { summary because } \\
\text { few studies mea- } \\
\text { sured this outcome }\end{array}$ \\
\hline & TSF & $\begin{array}{l}\text { Stated as an outcome mea- } \\
\text { sure in methodology, then not } \\
\text { reported in results }\end{array}$ & $\begin{array}{l}\text { Yes, for mean and } \\
\text { SD between groups }\end{array}$ & No response & $\begin{array}{l}\text { Not reported in the } \\
\text { summary because } \\
\text { few studies mea- } \\
\text { sured this outcome }\end{array}$ \\
\hline $\begin{array}{l}\text { Simmons } \\
2008\end{array}$ & Weight & $\begin{array}{l}\text { Data presented as phase } 1 \text { and } \\
2 \text { cross-over combined. The } \\
\text { data from phase } 1 \text { was needed } \\
\text { for this review }\end{array}$ & $\begin{array}{l}\text { Yes, for the phase } 1 \\
\text { data }\end{array}$ & $\begin{array}{l}\text { Yes, responded } \\
\text { but unable to } \\
\text { provide data }\end{array}$ & $\begin{array}{l}\text { Data reported in } \\
\text { structured narrative } \\
\text { summary }\end{array}$ \\
\hline
\end{tabular}


Table 8. Reasons for contacting authors, and outcomes of contact with authors (Continued)

\begin{tabular}{|c|c|c|c|c|c|}
\hline & $\mathrm{BMI}$ & $\begin{array}{l}\text { Data presented as phase } 1 \text { and } \\
2 \text { cross-over combined. The } \\
\text { data from phase } 1 \text { was needed } \\
\text { for this review }\end{array}$ & $\begin{array}{l}\text { Yes, for the phase } 1 \\
\text { data }\end{array}$ & $\begin{array}{l}\text { Yes, responded } \\
\text { but unable to } \\
\text { provide data }\end{array}$ & $\begin{array}{l}\text { Not reported in the } \\
\text { summary because } \\
\text { few studies mea- } \\
\text { sured this outcome }\end{array}$ \\
\hline & Energy intake & $\begin{array}{l}\text { Presented as pre- and post in- } \\
\text { tervention }\end{array}$ & $\begin{array}{l}\text { Yes, for mean and } \\
\text { SD of change }\end{array}$ & $\begin{array}{l}\text { Yes, responded } \\
\text { but unable to } \\
\text { provide data }\end{array}$ & $\begin{array}{l}\text { Imputed SD from Nijs } \\
2006\end{array}$ \\
\hline $\begin{array}{l}\text { Simmons } \\
2010\end{array}$ & Energy & $\begin{array}{l}\text { Reported as mean difference } \\
\text { without the SD }\end{array}$ & $\begin{array}{l}\text { Yes, requested SD } \\
\text { for mean change }\end{array}$ & $\begin{array}{l}\text { Yes, responded } \\
\text { but unable to } \\
\text { provide data }\end{array}$ & $\begin{array}{l}\text { Imputed SD from Nijs } \\
2006\end{array}$ \\
\hline \multicolumn{6}{|c|}{ 5. Home meal delivery systems } \\
\hline Kretser 2003 & Weight & $\begin{array}{l}\text { Reported separately for partic- } \\
\text { ipants at risk of malnutrition, } \\
\text { and those malnourished }\end{array}$ & $\begin{array}{l}\text { No, failed to find } \\
\text { contact information } \\
\text { for the author }\end{array}$ & $\mathrm{N} / \mathrm{A}$ & $\begin{array}{l}\text { Combined the mean } \\
\text { change data using } \\
\text { the formulae for } \\
\text { combining groups }\end{array}$ \\
\hline
\end{tabular}

ADL: activities of daily living; BMI: body mass index; $\mathrm{CI}$ : confidence interval; EuroQol: European Quality of Life Scale; IQR: interquartile range; MAC: midarm muscle circumference; N/A: not applicable; PEM: protein-energy malnutrition; SD: standard deviation; SGA: subjective global assessment; TSF: triceps skinfold thickness

Table 9. No. participants identified in each setting from included studies

\begin{tabular}{lll}
\hline Setting & $\begin{array}{l}\text { No. participants } \\
{[\mathbf{N} / \mathbf{N}(\%)]}\end{array}$ & No. studies \\
\hline Hospital & $7591 / 10,681(71.1)$ & 15 \\
\hline Residential care home & $1731 / 10,681(16.2)$ & 21 \\
\hline Free-living/outpatient setting & $1305 / 10,681(12.2)$ & 5 \\
\hline
\end{tabular}

Table 10. Effects of changes to the organisation of nutritional care on nutritional intake

\begin{tabular}{|c|c|c|c|c|c|}
\hline & \multirow[t]{2}{*}{ Outcome } & \multirow[t]{2}{*}{$(\mathrm{N})$} & \multicolumn{2}{|l|}{ Results } & \multirow[t]{2}{*}{ P Value } \\
\hline & & & Intervention & Control & \\
\hline \multicolumn{6}{|c|}{ Dietetic assistants (Hospital) } \\
\hline Duncan 2006 & $\begin{array}{l}\text { Mean (SD) energy intake } \\
\text { (kcal/day) }\end{array}$ & $\begin{array}{l}275(\text { total } N= \\
302)\end{array}$ & $1105(361)$ & $756(399)$ & $<0.001$ \\
\hline Hickson 2004 & $\begin{array}{l}\text { Between-group difference } \\
\text { (kcal) }\end{array}$ & $\begin{array}{l}37(\text { total } N= \\
592)\end{array}$ & 89 & & 0.538 \\
\hline \multicolumn{6}{|c|}{ Specialist training (residential care settings) } \\
\hline \multirow[t]{2}{*}{ Chang 2005} & $\%(\mathrm{SD})$ meals consumed & 67 & Pre: $90 \%(22)$ & Pre: $78 \%(34)$ & 0.49 \\
\hline & & & Post: 85 (25) & Post: $94 \%$ (18) & \\
\hline
\end{tabular}


Table 10. Effects of changes to the organisation of nutritional care on nutritional intake (Continued)

\begin{tabular}{|c|c|c|c|c|c|}
\hline \multirow[t]{6}{*}{ Lin 2010} & \multirow[t]{6}{*}{$\%(\mathrm{SD})$ meals consumed } & \multirow[t]{6}{*}{85} & Spaced retrieval (SR) & Pre: $79 \%$ (19) & SR vs control \\
\hline & & & Pre: $85 \%$ (11) & Post: $88 \%$ (18) & $=N S$ \\
\hline & & & Post: $91 \%$ (9) & & $\begin{array}{l}\text { MON vs con- } \\
\text { trol }\end{array}$ \\
\hline & & & Montessori (MON) & & \\
\hline & & & Pre: $75 \%$ (23) & & $<0.05$ \\
\hline & & & Post $78 \%$ (10) & & \\
\hline
\end{tabular}

\section{Multi-disciplinary team (hospital)}

\begin{tabular}{lllll}
\hline $\begin{array}{l}\text { Johansen } \\
2004\end{array}$ & $\begin{array}{l}\text { kcal/kg body weight per } \\
\text { day (SE) }\end{array}$ & $\begin{array}{l}202 \text { (total N }= \\
212)\end{array}$ & $25($ SE 1$)$ & $<0.005$ \\
\hline
\end{tabular}

kcal: kilocalorie; SD: standard deviation; SE: standard error

Table 11. Effects of changes to organisation of nutritional care on health-related quality of life, patient satisfaction and morbidity and complications

\begin{tabular}{|c|c|c|c|c|c|}
\hline & \multirow[t]{2}{*}{ Outcome } & \multirow[t]{2}{*}{ (N) } & \multicolumn{2}{|l|}{ Results } & \multirow[t]{2}{*}{ P Value } \\
\hline & & & Intervention & Control & \\
\hline \multicolumn{6}{|c|}{ Patient satisfaction } \\
\hline \multicolumn{6}{|c|}{ Dietetic assistants (hospital) } \\
\hline Duncan 2006 & Median score (IQR) & 159 & $6.5(2)$ & $3.0(4)$ & 0.0001 \\
\hline \multicolumn{6}{|c|}{ Health-related quality of life } \\
\hline \multicolumn{6}{|c|}{ Multi-disciplinary team (hospital) } \\
\hline \multirow{2}{*}{$\begin{array}{l}\text { Johansen } \\
2004\end{array}$} & Change in physical score (SF-36) & 110 & $2.4(1.3)$ & $0.2(1.5)$ & NS \\
\hline & Change in mental score (SF-36) & 110 & $2.2(2.5)$ & $3.3(2)$ & NS \\
\hline \multicolumn{6}{|c|}{ Number of complications } \\
\hline \multicolumn{6}{|c|}{ Dietetic assistants (hospital) } \\
\hline Duncan 2006 & $\begin{array}{l}\text { Total number of participants with compli- } \\
\text { cations }\end{array}$ & 302 & $84 / 125(67 \%)$ & $79 / 130(61 \%)$ & 0.29 \\
\hline Hickson 2004 & $\begin{array}{l}\text { Number of participants receiving oral an- } \\
\text { tibiotics }\end{array}$ & 592 & $142 / 292(49 \%)$ & $150 / 300(50 \%)$ & 0.67 \\
\hline \multicolumn{6}{|c|}{ Multi-disciplinary team (hospital) } \\
\hline $\begin{array}{l}\text { Johansen } \\
2004\end{array}$ & $\begin{array}{l}\text { Total number of participants with compli- } \\
\text { cations }\end{array}$ & 212 & $34 / 108(31 \%)$ & $23 / 10422 \%)$ & NS \\
\hline
\end{tabular}


Table 11. Effects of changes to organisation of nutritional care on health-related quality of life, patient satisfaction and morbidity and complications (Continued)
Olofsson 2007
Total number of participants with compli-
cations

IQR: interquartile range; NS: not significant; SF-36: short form-36

Table 12. Effects of changes to organisation of nutrition care on nutritional status

\begin{tabular}{|c|c|c|c|c|c|}
\hline & \multirow[t]{2}{*}{ Outcome } & \multirow[t]{2}{*}{ (N) } & \multicolumn{2}{|l|}{ Results } & \multirow[t]{2}{*}{ P Value } \\
\hline & & & Intervention & Control & \\
\hline \multicolumn{6}{|c|}{ Dietetic assistants (hospital) } \\
\hline \multirow[t]{4}{*}{ Duncan 2006} & Mean change (SD) & (total $N=302$ ) & $-0.36(3.3)$ & $-1.0(2.8)$ & 0.16 \\
\hline & Weight (kg) & 170 & $-0.9(2.2)$ & $-1.3(1.5)$ & 0.002 \\
\hline & $\mathrm{MAC}(\mathrm{cm})$ & 230 & $-0.88(2.6)$ & $-1.23(3.2)$ & 0.087 \\
\hline & $\mathrm{TSF}(\mathrm{mm})$ & 205 & & & \\
\hline \multirow[t]{7}{*}{ Hickson 2004} & Mean change (SD) & (total $N=592)$ & $-0.92(2.71)$ & $-0.9(3)$ & 0.23 \\
\hline & Weight (kg) & 191 & $-0.3(1)$ & $-0.3(1)$ & 0.65 \\
\hline & $\operatorname{MAC}(\mathrm{cm})$ & 286 & $-0.4(1.8)$ & $-0.4(1.7)$ & 0.86 \\
\hline & $\mathrm{TSF}(\mathrm{mm})$ & 279 & $-0.1(-0.8-0.4)$ & $-0.1(-0.5-0.3)$ & 0.84 \\
\hline & Median (IQR) & 429 & $-0.04(1.1)$ & $-0.25(1.18)$ & 0.68 \\
\hline & MAMC & 254 & & & \\
\hline & BMI $\left(\mathrm{kg} / \mathrm{m}^{2}\right)$ & & & & \\
\hline
\end{tabular}

Specialist training (residential care settings)

\begin{tabular}{|c|c|c|c|c|c|}
\hline $\operatorname{Lin} 2010$ & $\begin{array}{l}\text { Mean change (SD) } \\
\text { Weight }(\mathrm{kg}) \\
\text { BMI }\left(\mathrm{kg} / \mathrm{m}^{2}\right)\end{array}$ & 85 & $\begin{array}{l}\text { Spaced retrieval } \\
-0.07(0.57) \\
\text { Montessori } \\
-0.15(0.57) \\
\text { Spaced retrieval } \\
0.1(1.0) \\
\text { Montessori } \\
-0.06(1.0)\end{array}$ & $\begin{array}{l}-0.09(0.57) \\
-0.03(1)\end{array}$ & $\begin{array}{l}\text { NS } \\
\text { NS }\end{array}$ \\
\hline Lin 2011 & BMI & 29 & $-0.26(0.73)$ & $-0.09(0.85)$ & 0.245 \\
\hline \multicolumn{6}{|c|}{ Specialist training (free-living individuals) } \\
\hline Pivi 2011 & $\begin{array}{l}\text { Mean change (SD) } \\
\text { Weight }(\mathrm{kg})\end{array}$ & 52 & $\begin{array}{l}1.19 \text { (imputed SD: 3.3) } \\
1.87 \text { (2) }\end{array}$ & $\begin{array}{l}-2.2 \text { (imputed SD: } \\
3.3 \text { ) }\end{array}$ & $\begin{array}{l}\text { Report- } \\
\text { ed as be- } \\
\text { tween-group }\end{array}$ \\
\hline
\end{tabular}


Table 12. Effects of changes to organisation of nutrition care on nutritional status (Continued)
MAC $(\mathrm{cm})$
2.3 (5.4)
$-0.4(0.46)$
TSF (mm)
1.19 (1)
$2.2(5.3)$
$\operatorname{BMI}\left(\mathrm{kg} / \mathrm{m}^{2}\right)$
$-2.21(1)$

differences for

\begin{tabular}{|c|c|c|c|c|c|}
\hline \multirow[t]{2}{*}{ Salva 2011} & Mean change (SD) & 946 & $0.26(0.7)$ & $0.09(0.5)$ & 0.598 \\
\hline & Weight (kg) & & $-0.01(2.2)$ & $-0.06(3.2)$ & 0.843 \\
\hline
\end{tabular}

\begin{tabular}{|c|c|c|c|c|c|}
\hline \multicolumn{6}{|c|}{ Multi-disciplinary team (hospital) } \\
\hline \multirow{2}{*}{$\begin{array}{l}\text { Johansen } \\
2004\end{array}$} & Mean change (SD) & $($ total $N=212)$ & $-0.22(3.9)$ & $0.1(2)$ & NS \\
\hline & Weight (kg) & 95 & & & \\
\hline \multirow[t]{3}{*}{ Olofsson 2007} & Mean change (SD) & (total $N=199$ ) & $-1.1(3.6)$ & $-0.7(3.8)$ & 0.05 \\
\hline & Weight (kg) & 157 & $-0.45(1.3)$ & $-0.3(1.5)$ & 0.05 \\
\hline & BMI $\left(\mathrm{kg} / \mathrm{m}^{2}\right)$ & 157 & & & \\
\hline
\end{tabular}

\section{Protocol-driven pathway (hospital)}

\begin{tabular}{|c|c|c|c|c|c|}
\hline \multirow[t]{2}{*}{ Holyday 2012} & Mean change (SD) & (total $N=143$ ) & $-0.9(3.6)$ & $-0.9(2.3)$ & 0.98 \\
\hline & Weight (kg) & 69 & & & \\
\hline
\end{tabular}

\section{Protocol-driven pathway (residential care settings)}

\begin{tabular}{|c|c|c|c|c|}
\hline Splett 2003 & Weight & 364 & $\begin{array}{l}\text { No wt loss at baseline: } 95 \% \\
\text { maintained wt. } \\
\text { Wt loss at baseline: } 48 \% \\
\text { maintained or gained wt. }\end{array}$ & $\begin{array}{l}\text { No wt loss at base- } \\
\text { line: } 58 \% \text { maintained } \\
\text { wt. } \\
\text { Wt loss at baseline: } \\
57 \% \text { maintained or } \\
\text { gained wt. }\end{array}$ \\
\hline
\end{tabular}

Telemedicine (free-living individuals)

\begin{tabular}{llllll}
\hline Kraft 2012 & Mean change (SD) & 26 & $-4.5(7.9)$ & $-3(6.2)$ & NS \\
& Weight $(\mathrm{kg})$ & 14 & Baseline 24.5 (5.1) & Baseline 23.9 (4.4) & NS \\
& BMI $\left(\mathrm{kg} / \mathrm{m}^{2}\right)$ & & Follow-up 23.0 (4.2) & Follow-up 22.8 (4.3) \\
\hline
\end{tabular}

BMI: body mass index; IQR: interquartile range; MAC: mid-arm circumference; MAMC: mid-arm muscle circumference; NS: not significant; SD: standard deviation; TSF: triceps skinfold thickness; wt: weight

Table 13. Effects of changes to the organisation of nutritional care on handgrip strength

\begin{tabular}{llll} 
Outcome & $(\mathrm{N})$ & Results & Control \\
\cline { 3 - 4 } & & & \\
\hline Handervention & \\
\hline
\end{tabular}


Table 13. Effects of changes to the organisation of nutritional care on handgrip strength (Continued)

\section{Dietetic assistants (Hospital)}

\begin{tabular}{llllll}
\hline Duncan 2006 & Mean change (SD) & 126 (total N = 302) & $2.2(10.7)$ & $0.16(11.8)$ & 0.32 \\
\hline Hickson 2004 & Median change (IQR) $(\mathrm{kg})$ & (total N = 592) & $0.8(-1.4$ to 2.5) & $0.7(-1.5$ to 3) & 0.85 \\
\hline
\end{tabular}

IQR: interquartile range; SD: standard deviation

Table 14. Effects of changes to the organisation of nutritional care on hospitalisation, institutionalisation and death from any cause

Outcome

(N)
Results

Intervention

\section{P Value}

\section{Control}

\section{Mortality}

Dietetic assistants (Hospital)

\begin{tabular}{|c|c|c|c|c|c|}
\hline Duncan 2006 & 4-month mortality & (total $N=302$ ) & $19 / 145(13 \%)$ & $36 / 157(23 \%)$ & 0.036 \\
\hline Hickson 2004 & In-hospital mortality & (total $N=592$ ) & $31 / 292(11 \%)$ & $35 / 300(12 \%)$ & 0.69 \\
\hline
\end{tabular}

Specialist training (free-living individuals)

\begin{tabular}{lccccc}
\hline Salva 2011 & 12-month mortality & 946 & $43 / 448(10 \%)$ & $29 / 498(6 \%)$ & NR \\
\hline \multicolumn{2}{l}{ Multi-disciplinary team (hospital) } & & & \\
\hline Olofsson 2007 & 4-month mortality & 199 & $9 / 102(9 \%)$ & $13 / 97(13 \%)$ & NR \\
\hline $\begin{array}{l}\text { Protocol-driven pathway (hospital) } \\
\text { Holyday 2012 Not reported }\end{array}$ & 143 & $1 / 72(1 \%)$ & $4 / 71(6 \%)$ & 0.21
\end{tabular}

\section{Length of stay in hospital}

\begin{tabular}{|c|c|c|c|c|c|}
\hline \multicolumn{6}{|c|}{ Dietetic assistants (hospital) } \\
\hline Duncan 2006 & Median (IQR) (days) & 167 & $34(48)$ & $32(49)$ & 0.81 \\
\hline Hickson 2004 & Median (IQR) (days) & 592 & $21(13-36)$ & $23(14-39)$ & 0.41 \\
\hline \multicolumn{6}{|c|}{ Multi-disciplinary team (hospital) } \\
\hline \multirow[t]{2}{*}{ Johansen 2004} & Mean (SD) & 197 & $11.6(8)$ & $11.5(8)$ & NS \\
\hline & LOS to 28 days & & & & \\
\hline Olofsson 2007 & Mean (SD) (days) & 157 & $27.4(15.9)$ & $39.8(41.9)$ & $<0.05$ \\
\hline \multicolumn{6}{|c|}{ Protocol-driven pathway (hospital) } \\
\hline Holyday 2012 & Mean (SD) (days) & 143 & $13.7(11.8)$ & $13.5(11)$ & 0.85 \\
\hline
\end{tabular}


Table 14. Effects of changes to the organisation of nutritional care on hospitalisation, institutionalisation and death from any cause (Continued)

Hospital readmissions

\section{Protocol-driven pathway (hospital)}

\begin{tabular}{llll}
\hline Holyday 2012 & $\begin{array}{l}\text { Number of readmissions at } 6 \\
\text { months }\end{array}$ & $30 / 71$ & $37 / 72$
\end{tabular}

IQR: interquartile range; LOS: length of stay; SD: standard deviation

Table 15. Effects of changes to the feeding environment on nutritional intake

\begin{tabular}{|c|c|c|c|c|c|c|}
\hline & \multirow[t]{2}{*}{ Outcome } & \multirow[t]{2}{*}{ (N) } & \multicolumn{2}{|l|}{ Results } & \multicolumn{2}{|l|}{ P Value } \\
\hline & & & $\begin{array}{l}\text { Interven- } \\
\text { tion }\end{array}$ & Control & & \\
\hline \multicolumn{7}{|c|}{ Changes to the dining room environment } \\
\hline Mathey 2001 & $\begin{array}{l}\text { Mean change (SD) energy intake } \\
\text { (kcal) }\end{array}$ & 22 & $199(406)$ & $185(247)$ & NR & \\
\hline \multirow[t]{2}{*}{ Nijs 2006} & $\begin{array}{l}\text { Mean change (SD) energy intake } \\
\text { (kcal) }\end{array}$ & 178 & $116(456)$ & $-100(357)$ & & \\
\hline & Mean difference $(95 \% \mathrm{Cl})$ & 178 & $235(83-268)$ & & & $\begin{array}{l}\text { Described as } \\
\text { significantly } \\
\text { different } \\
\text { but no P value } \\
\text { reported }\end{array}$ \\
\hline $\begin{array}{l}\text { Remsburg } \\
2001\end{array}$ & NR & & & & & \\
\hline \multicolumn{7}{|c|}{ Sensory stimulation } \\
\hline $\begin{array}{l}\text { Brouillette } \\
1991\end{array}$ & $\begin{array}{l}\text { Mean change (SD) in intake of lunch } \\
\text { meal (kcal) }\end{array}$ & 16 & $-1.6(450)$ & $11.14(360)$ & 0.49 & \\
\hline
\end{tabular}

CI: confidence interval; NR: not reported; SD: standard deviation

Table 16. Effects of changes to the feeding environment on health related quality of life

\begin{tabular}{|c|c|c|c|c|c|}
\hline & \multirow[t]{2}{*}{ Outcome } & \multirow[t]{2}{*}{ (N) } & \multicolumn{2}{|l|}{ Results } & \multirow[t]{2}{*}{ P Value } \\
\hline & & & Intervention & Control & \\
\hline \multicolumn{6}{|c|}{ Changes to the dining room environment } \\
\hline Mathey 2001a & $\begin{array}{l}\text { Sickness Impact Profile, mean } \\
\text { change (SD) in score }\end{array}$ & $16 / 2$ & $-2(11)$ & $-13(12)$ & NR \\
\hline
\end{tabular}


Table 16. Effects of changes to the feeding environment on health related quality of life (Continued)

\begin{tabular}{|c|c|c|c|c|c|}
\hline \multirow[t]{4}{*}{ Nijs 2006} & $\begin{array}{l}\text { Overall QOL mean change }(95 \% \mathrm{Cl}) \\
\text { in score }\end{array}$ & 178 & $\begin{array}{l}0.4(-1.8 \text { to } \\
2.5)\end{array}$ & $-5(-9.4$ to -0.6$)$ & NR \\
\hline & Mean difference $(95 \% \mathrm{Cl})$ & 178 & 6.1 (2.1 to 10.3$)$ & & $\begin{array}{l}\text { Described as signifi- } \\
\text { cantly different } \\
\text { but no P value reported }\end{array}$ \\
\hline & $\begin{array}{l}\text { Physical performance, mean } \\
\text { change }(95 \% \mathrm{Cl}) \text { in score }\end{array}$ & 178 & $\begin{array}{l}0.2(-2.3 \text { to } \\
2.7)\end{array}$ & $\begin{array}{l}-2.2(-4.1 \text { to } \\
-0.4)\end{array}$ & NR \\
\hline & Mean difference $(95 \% \mathrm{Cl})$ & 178 & 3.20 .9 to 5.5$)$ & & $\begin{array}{l}\text { Described as signifi- } \\
\text { cantly different } \\
\text { but no P value reported }\end{array}$ \\
\hline
\end{tabular}

Cl: confidence interval; NR: not reported; QOL: quality of life; SD: standard deviation

Table 17. Effects of changes to the feeding environment on nutritional status

\begin{tabular}{|c|c|c|c|c|c|}
\hline & \multirow[t]{2}{*}{ Outcome } & \multirow[t]{2}{*}{ (N) } & \multicolumn{2}{|l|}{ Results } & \multirow[t]{2}{*}{ P Value } \\
\hline & & & Intervention & Control & \\
\hline \multicolumn{6}{|l|}{ Weight } \\
\hline \multicolumn{6}{|c|}{ Changes to the dining room environment } \\
\hline Mathey 2001a & Mean change (SD) (kg) & 22 & $3.3(5)$ & $-0.4(4)$ & $\mathrm{I}:<0.05 ; \mathrm{C}: 0.78$ \\
\hline \multirow[t]{3}{*}{ Nijs 2006} & Mean change (SD) (kg) & 178 & $0.5(3.9)$ & $-1.1(3.7)$ & NR \\
\hline & Mean difference $(95 \% \mathrm{Cl})$ & 178 & 1.5 (0.6 to 2.4$)$ & & $\begin{array}{l}\text { Described as significantly } \\
\text { different }\end{array}$ \\
\hline & & & & & but no $P$ value reported \\
\hline $\begin{array}{l}\text { Remsburg } \\
2001\end{array}$ & Mean change (SD) (kg) & 39 & $-0.11(3.1)$ & $0.32(2.2)$ & 0.638 \\
\hline
\end{tabular}

C: control; I: intervention; NR: not recorded; SD: standard deviation

Table 18. Effects of changes to the feeding environment on death from any cause

\begin{tabular}{llllll}
\hline \multicolumn{2}{c}{ Outcome } & (N) & \multicolumn{2}{l}{ Results } & P Value \\
\cline { 3 - 5 } & & Intervention & Control \\
\hline \multicolumn{2}{l}{ Changes to the dining room environment } & & & & \\
\hline Mathey 2001a & Mortality & 38 & $7 / 21(33 \%)$ & $5 / 17(29 \%)$ & $\mathrm{NR}$ \\
\hline Nijs 2006 & Mortality & 178 & $18 / 112(16 \%)$ & $16 / 133(12 \%)$ & $\mathrm{NR}$ \\
\hline
\end{tabular}

\section{Sensory stimulation}


Table 18. Effects of changes to the feeding environment on death from any cause (Continued)
Brouillette 1991
Mortality
20
$1 / 10(10 \%)$
$0 / 10(0 \%)$

NR

NR: not reported 


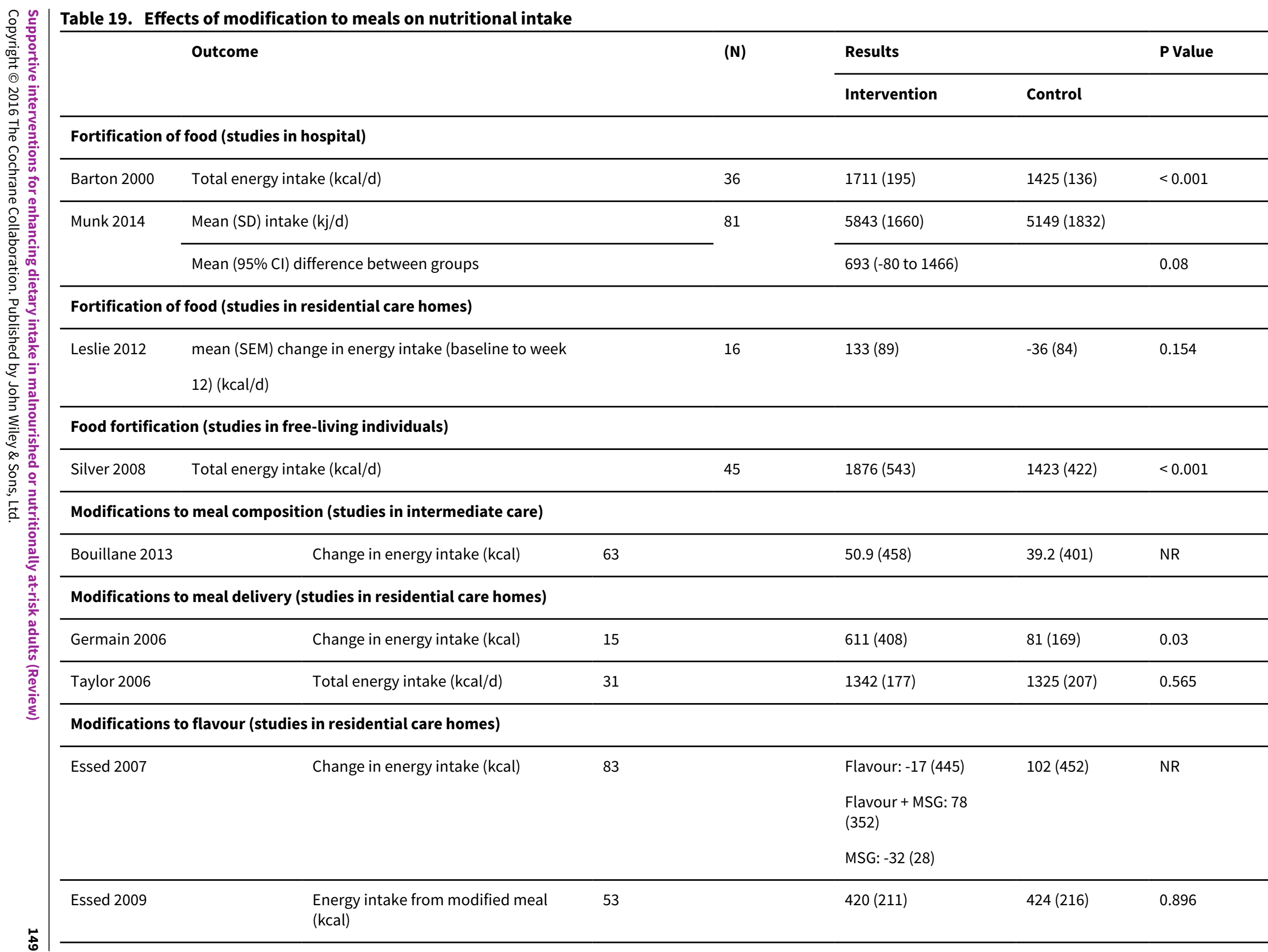




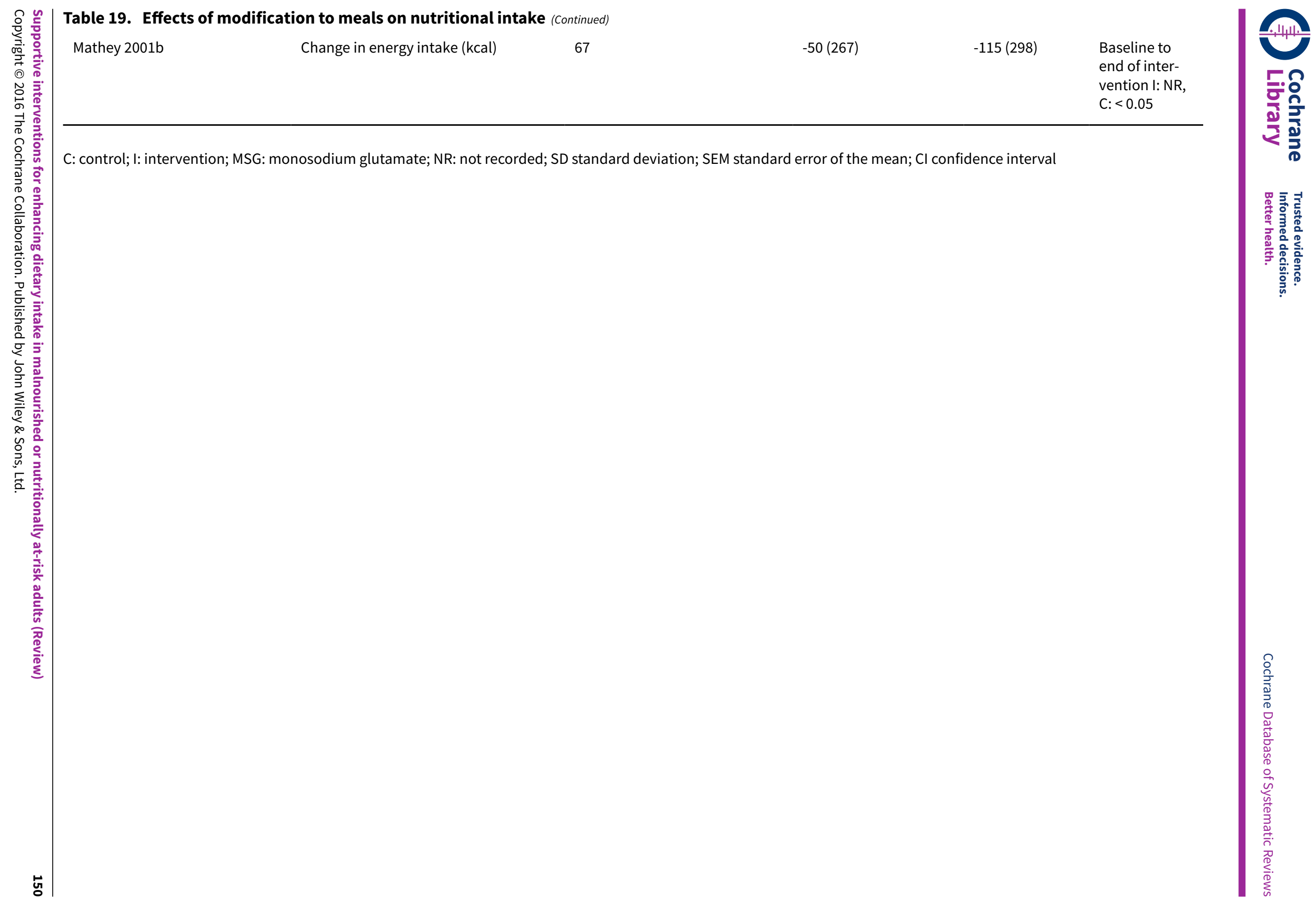


Table 20. Effects of modifications to meals on nutritional status

\begin{tabular}{|c|c|c|c|c|c|}
\hline & \multirow[t]{2}{*}{ Outcome } & \multirow[t]{2}{*}{ (N) } & \multicolumn{2}{|l|}{ Results } & \multirow[t]{2}{*}{ P Value } \\
\hline & & & Intervention & Control & \\
\hline \multicolumn{6}{|c|}{ Weight and BMI (mean change (SD)) } \\
\hline \multicolumn{6}{|c|}{ Fortification of food (studies in hospital) } \\
\hline \multirow[t]{2}{*}{ Munk 2014} & $\begin{array}{l}\text { Mean (SD) within-group change in } \\
\text { weight }(\mathrm{kg})\end{array}$ & 66 & $0.4(2.6)$ & $-0.4(1.8)$ & 0.17 \\
\hline & $\begin{array}{l}\text { Mean }(95 \% \mathrm{Cl}) \text { between-group differ- } \\
\text { ence in } \\
\text { weight }(\mathrm{kg})\end{array}$ & & $-0.8(-1.9$ to 0.3$)$ & & \\
\hline
\end{tabular}

\section{Fortification of food (studies in residential care homes)}

\begin{tabular}{|c|c|c|c|c|c|}
\hline \multirow[t]{3}{*}{ Leslie 2012} & $\begin{array}{l}\text { Mean (SD) within-group weight change } \\
(\mathrm{kg})\end{array}$ & 31 & $1.3(0.53)^{\star}$ & $-0.2(1.5)^{\star *}$ & $\begin{array}{l}{ }^{*} 0.03 \\
{ }^{\star *} 0.536\end{array}$ \\
\hline & $\begin{array}{l}\text { Mean (SD) within-group change in BMI } \\
\left(\mathrm{kg} / \mathrm{m}^{2}\right)\end{array}$ & 31 & $0.5(0.25)^{\star}$ & $-0.1(0.4)^{\star \star}$ & $\begin{array}{l}{ }^{\star} 0.042 \\
{ }^{\star \star} 0.517\end{array}$ \\
\hline & $\begin{array}{l}\text { Mean (SD) within-group change in MUAC } \\
(\mathrm{mm})\end{array}$ & 32 & $0.4(0.16)^{*}$ & $-0.1(0.3)^{\star \star}$ & $\begin{array}{l}{ }^{\star} 0.019 \\
{ }^{\star \star} 0.691\end{array}$ \\
\hline \multirow{2}{*}{$\begin{array}{l}\text { Smolliner } \\
2008\end{array}$} & Mean (SD) change weight (kg) & 52 & $2(2.1)$ & $1.6(2)$ & NS \\
\hline & BMI change $\left(\mathrm{kg} / \mathrm{m}^{2}\right)$ & 52 & $0.77(1.5)$ & $0.45(1.1)$ & $\begin{array}{l}\text { Be- } \\
\text { tween-group } \\
\text { difference NS }\end{array}$ \\
\hline
\end{tabular}

\section{Modifications to meal composition (studies in intermediate care)}

\begin{tabular}{llllll}
\hline $\begin{array}{l}\text { Bouillanne } \\
2013\end{array}$ & Mean (SD) change weight (kg) & 63 & $0.4(2.3)$ & $-0.7(3.1)$ & NR \\
\hline
\end{tabular}

\section{Modifications to meal delivery (studies in residential care homes)}

\begin{tabular}{|c|c|c|c|c|c|}
\hline \multirow[t]{2}{*}{ Germain 2006} & Mean (SD) change weight (kg) & 15 & $3.9(2.3)$ & $-0.8(4.2)$ & 0.02 \\
\hline & BMI change $\left(\mathrm{kg} / \mathrm{m}^{2}\right)$ & 15 & $1.51(1.16)$ & $0.27(1.46)$ & $\begin{array}{l}\text { Data provided } \\
\text { by } \\
\text { study author } \mathrm{P} \\
\text { value NR }\end{array}$ \\
\hline
\end{tabular}

Modifications to flavour (studies in residential care homes)

\begin{tabular}{lllllll}
\hline Essed 2007 & Mean (SD) change weight (kg) & 83 & Flavour: 0.1 (2.4) & $0.1(3.8)$ & NR
\end{tabular}


Table 20. Effects of modifications to meals on nutritional status (Continued)

Flavour + MSG: -
$0.8(3.3)$

MSG: - 0.7 (3.6)

\begin{tabular}{llllll}
\hline Mathey 2001b & Mean (SD) change weight (kg) & 67 & $1.1(1.3)$ & $-0.3(1.6)$ & $<0.05$ \\
\hline
\end{tabular}

BMI: body mass index; CI: confidence interval; MSG: monosodium glutamate; MUAC: mid-upper arm circumference; NR: not reported; NS: not significant; SD: standard deviation

Table 21. Effects of modifications to meals on clinical function, hospitalisation and death from any cause

\begin{tabular}{|c|c|c|c|c|c|}
\hline & \multirow[t]{2}{*}{ Outcome } & \multirow[t]{2}{*}{ (N) } & \multicolumn{2}{|l|}{ Results } & \multirow[t]{2}{*}{ P Value } \\
\hline & & & Intervention & Control & \\
\hline \multicolumn{6}{|l|}{ Mortality } \\
\hline \multicolumn{6}{|c|}{ Fortification of food (studies in hospital) } \\
\hline Munk 2014 & Mortality & 81 & $1 / 44$ & $1 / 40$ & NR \\
\hline \multicolumn{6}{|c|}{ Fortification of food (studies in residential care homes) } \\
\hline Leslie 2012 & Mortality & 32 & $2 / 19$ & $5 / 22$ & NR \\
\hline Smolliner 2008 & Mortality & 65 & $2 / 31$ & $1 / 34$ & NR \\
\hline \multicolumn{6}{|c|}{ Modifications to meal composition (studies in intermediate care) } \\
\hline Bouillane 2013 & Mortality & 66 & $1 / 30(3 \%)$ & $1 / 36(3 \%)$ & NR \\
\hline \multicolumn{6}{|c|}{ Length of hospital stay } \\
\hline \multicolumn{6}{|c|}{ Fortification of food (studies in hospital) } \\
\hline Munk 2014 & $\begin{array}{l}\text { Days from study inclusion to dis- } \\
\text { charge }\end{array}$ & 81 & $10(8)$ & $10(8)$ & 0.73 \\
\hline \multicolumn{6}{|c|}{ Handgrip strength } \\
\hline \multicolumn{6}{|c|}{ Fortification of food (studies in hospital) } \\
\hline \multirow[t]{2}{*}{ Munk 2014} & $\begin{array}{l}\text { Mean change (SD) baseline to day } 3 \\
(\mathrm{~kg})\end{array}$ & 76 & $-0.1(2.9)$ & $-0.4(4.3)$ & 0.76 \\
\hline & $\begin{array}{l}\text { Mean difference }(95 \% \mathrm{CI}) \text { between I \& } \\
\mathrm{C}\end{array}$ & & $-0.3(-1.9$ to -1.4$)$ & & 0.95 \\
\hline
\end{tabular}

\section{Fortification of food (studies in residential care homes)}

\begin{tabular}{llllll}
\hline Smolliner 2008 & Mean change (SD) (kg) & 61 & $-0.81(3.12)$ & $-1.29(3)$ & NR \\
\hline
\end{tabular}

\section{Modifications to meal composition (studies in intermediate care)}


Table 21. Effects of modifications to meals on clinical function, hospitalisation and death from any cause (Continued)

\begin{tabular}{|c|c|c|c|c|c|}
\hline Bouillane 2013 & Mean change (SD) (N) & 63 & $-0.5(41.7)$ & $14(45.1)$ & $\begin{array}{l}0.411 \text { (ANCO- } \\
\text { VA } 0.271 \text { ) }\end{array}$ \\
\hline Bouillane 2013 & Change in ADL score (mean (SD) & 63 & $-0.02(1.6)$ & $0.54(1.7)$ & $\begin{array}{l}0.125 \text { (ANCO- } \\
\text { VA } 0.118 \text { ) }\end{array}$ \\
\hline
\end{tabular}

ADL: activities of daily living; ANCOVA: analysis of covariance; N: Newtons; NR: not reported; SD: standard deviation

I: intervention; C: control

Table 22. Effects of supplementation of meals on nutritional intake

\begin{tabular}{|c|c|c|c|c|c|}
\hline & \multirow[t]{2}{*}{ Outcome } & \multirow[t]{2}{*}{ (N) } & \multicolumn{2}{|l|}{ Results } & \multirow[t]{2}{*}{ P Value } \\
\hline & & & Intervention & Control & \\
\hline \multicolumn{6}{|c|}{ Supplementation with food (residential care homes) } \\
\hline Beck 2002 & $\begin{array}{l}\text { Change in energy intake }(\mathrm{kcal} / \mathrm{d}) \text { (me- } \\
\text { dian } 95 \% \mathrm{Cl} \text { ) }\end{array}$ & 16 & $-24(-454$ to 860$)$ & $\begin{array}{l}24(-167 \text { to } \\
478)\end{array}$ & NS \\
\hline Simmons 2008 & $\begin{array}{l}\text { Change in energy intake kcal/ (mean } \\
\text { SD) }\end{array}$ & 64 & $302(450)$ & $127(360)$ & $\begin{array}{l}\text { Baseline to } 6 \\
\text { months I: = } \\
0.000 ; \text { C: NS }\end{array}$ \\
\hline Simmons 2010 & Change in energy intake (mean SD) & 43 & $-65(450)$ & $67(360)$ & NS \\
\hline \multicolumn{6}{|c|}{ Supplementation with ONS (in hospital) (reported as mean (SD) } \\
\hline $\begin{array}{l}\text { Bourdel-Mar- } \\
\text { chasson } 2000\end{array}$ & Total energy intake (kcal/d) & 672 & $1188(613)$ & $1102(503)$ & 0.13 \\
\hline $\begin{array}{l}\text { Faxen-Irving } \\
2011\end{array}$ & Change in energy intake (kcal/d) & 38 & $94(350)$ & $6.5(358)$ & NR \\
\hline Potter 2001 & Total energy intake (kcal/d) & 381 & $1409(448)$ & $1090(417)$ & $\mathrm{S}$ \\
\hline $\begin{array}{l}\text { Van den Berg } \\
2015\end{array}$ & $\begin{array}{l}\text { Mean (SD) energy intake from ONS } \\
(\mathrm{kcal} / \mathrm{d})\end{array}$ & 192 & $\begin{array}{l}\text { I1:343 }(172)^{\star} \\
\text { I2: } 469(111)^{\star \star}\end{array}$ & $389(162)$ & $\begin{array}{l}{ }^{\star} 0.289 \\
{ }^{\star \star} 0.006\end{array}$ \\
\hline \multicolumn{6}{|c|}{ Supplementation with ONS (long-term/residential care settings) } \\
\hline Hankey 1993 & Total energy intake (kcal/d) & 21 & $1747(273)$ & $1147(310)$ & $\begin{array}{l}\text { Baseline to wk } \\
8,1: 0.01 ; C: N S\end{array}$ \\
\hline Simmons 2010 & Change in energy intake & 42 & $28(450)$ & $67(360)$ & 0.14 \\
\hline
\end{tabular}

C: control; Cl: confidence interval; I: intervention; NS: not significant; NR: not reported; ONS: oral nutritional supplement; S: significant; SD: standard deviation; wk: week

Table 23. Effects of supplementation of meals on health-related quality of life, morbidity/complications

\begin{tabular}{llll} 
Outcome & (N) & Results & P Value \\
\cline { 3 - 4 } & & Intervention Control
\end{tabular}


Table 23. Effects of supplementation of meals on health-related quality of life, morbidity/complications (Continued) Incidence of pressure ulcers

\section{Supplementation with ONS (in hospital)}

\begin{tabular}{llllll}
\hline $\begin{array}{l}\text { Bourdel-Mar- } \\
\text { chasson 2000 }\end{array}$ & $\begin{array}{l}\text { Cumulative incidence at end of follow-up } \\
\text { (\%) }\end{array}$ & 672 & 40 & 48 & NR \\
& $\begin{array}{l}\text { Number of participants with pressure ul- } \\
\text { cers at day 15 }\end{array}$ & $101 / 295$ & $164 / 37$ & NR \\
\hline Dennis 2005 & $\begin{array}{l}\text { Number of participants with pressure ul- } \\
\text { cers }\end{array}$ & 4023 & $15 / 2016$ & $26 / 2007$ & 0.0507 \\
\hline
\end{tabular}

\section{Total complications}

\section{Supplementation with ONS (in hospital)}

\begin{tabular}{lllll}
\hline Dennis 2005 & All in-hospital complications & 4023 & $515 / 2014(26 \%)$ & $\begin{array}{l}448 / 2001 \\
(22 \%)\end{array}$
\end{tabular}

\section{Health-related quality of life}

\section{Supplementation with ONS (in hospital)}

\begin{tabular}{llll}
\hline Dennis 2005 & Utilitiy (median (IQR)) (EUROQoL) & 3086 & $\begin{array}{l}\text { Median group difference } 0.52 \\
(0.03 \text { to 0.74) }\end{array}$
\end{tabular}

EUROQol: European Quality of Life Scale; IQR: interquartile range; NR: not reported; ONS: oral nutritional supplement

Table 24. Effects of supplementation of meals on nutritional status

\begin{tabular}{|c|c|c|c|c|c|}
\hline & \multirow[t]{2}{*}{ Outcome } & \multirow[t]{2}{*}{ (N) } & \multicolumn{2}{|l|}{ Results } & \multirow[t]{2}{*}{ P Value } \\
\hline & & & Intervention & Control & \\
\hline \multicolumn{6}{|c|}{ Supplementation with food (residential care homes) } \\
\hline Beck 2002 & Change in weight (median $95 \% \mathrm{Cl}$ ) & 16 & $1.3(-1$ to 3$)$ & $1.5(-2.3$ to 9$)$ & NS \\
\hline $\begin{array}{l}\text { Simmonds } \\
2008\end{array}$ & $\begin{array}{l}\text { Mean change (SD) weight }(\mathrm{kg}) \\
\text { Mean (SD) change in BMI }\end{array}$ & 64 & $\begin{array}{l}\text { The intervention } \\
\text { group gained } 4 \mathrm{lbs} \\
\text { more } \\
\text { The intervention } \\
\text { group gained } 0.72 \mathrm{~kg} / \\
\mathrm{m}^{2} \text { than the usual care }\end{array}$ & $\begin{array}{l}\text { NR } \\
\text { NR }\end{array}$ & $\begin{array}{l}0.009 \\
0.009\end{array}$ \\
\hline $\begin{array}{l}\text { Simmonds } \\
2010\end{array}$ & Mean change (SD) weight $(\mathrm{kg})$ & 43 & $0.02(1.1)$ & $0.21(1.7)$ & NS \\
\hline \multicolumn{6}{|c|}{ Supplementation with ONS (in hospital) } \\
\hline \multirow{2}{*}{$\begin{array}{l}\text { Faxen-Irving } \\
2011\end{array}$} & Mean change (SD) weight $(\mathrm{kg})$ & 38 & $0.13(2.2)$ & $-0.95(2.3)$ & NR \\
\hline & $\begin{array}{l}\text { Mean (SD) BMI at follow-up (kg/ } \\
\mathrm{m} 2)\end{array}$ & 38 & $20.4(3.7)$ & $\begin{array}{l}20.4(3.7) \\
21.9(3.8)\end{array}$ & 0.17 \\
\hline
\end{tabular}


Table 24. Effects of supplementation of meals on nutritional status (Continued)

\begin{tabular}{|c|c|c|c|c|c|}
\hline \multirow[t]{2}{*}{ Potter 2001} & Mean change in weight $(\mathrm{kg})$ & 381 & $0.4(2.6)$ & $-0.5(2.9)$ & 0.003 \\
\hline & Mean change (SD) MAC (cm) & 381 & $-0.1(1.3)$ & $-0.4(1.2)$ & NS \\
\hline \multicolumn{6}{|c|}{ Supplementation with ONS (long-term care settings) } \\
\hline \multirow[t]{3}{*}{ Hankey 1993} & Mean change (SD) weight $(\mathrm{kg})$ & 21 & $2.83(10)$ & $-0.53(10)$ & \multirow{3}{*}{$\begin{array}{l}\text { NR - data from } \\
\text { Milne } 2009 \\
\text { NR data from } \\
\text { Milne } 2009\end{array}$} \\
\hline & Mean change (SD) MAC & 21 & $-1(10)$ & $0.6(10)$ & \\
\hline & & & & & \\
\hline $\begin{array}{l}\text { Simmons } \\
2010\end{array}$ & Mean change in weight (kg) & 42 & $0.91(2.3)$ & $0.24(1.96)$ & NS \\
\hline
\end{tabular}

BMI: body mass index; $\mathrm{Cl}$ : confidence interval; MAC: mid-arm circumference; NR: not reported; NS: not significant; ONS: oral nutritional supplement; SD: standard deviation

Table 25. Effects of supplementation of meals on hospitalisation, institutionalisation and death from any cause

\begin{tabular}{|c|c|c|c|c|c|}
\hline & \multirow[t]{2}{*}{ Outcome } & \multirow[t]{2}{*}{ (N) } & \multicolumn{2}{|l|}{ Results } & \multirow[t]{2}{*}{ P Value } \\
\hline & & & Intervention & Control & \\
\hline \multicolumn{6}{|l|}{ Mortality } \\
\hline \multicolumn{6}{|c|}{ Supplementation with ONS (in hospital) } \\
\hline $\begin{array}{l}\text { Bourdel-Marchas- } \\
\text { son } 2000\end{array}$ & Mortality & 672 & $25 / 295(8 \%)$ & $22 / 377(6 \%)$ & 0.18 \\
\hline Dennis 2005 & Mortality & 4023 & $241 / 2016(12 \%)$ & $253 / 2007(13 \%)$ & 0.7 \\
\hline Potter 2001 & Mortality & 381 & $21 / 186(11 \%)$ & $33 / 195(17 \%)$ & 0.117 \\
\hline \multicolumn{6}{|c|}{ Supplementation with ONS (long-term care settings) } \\
\hline Larsson 1990 & Mortality & 435 & $29 / 197(15 \%)$ & $56 / 238(24 \%)$ & 0.13 \\
\hline \multicolumn{6}{|l|}{ Length of stay } \\
\hline \multicolumn{6}{|c|}{ Supplementation with ONS (in hospital) } \\
\hline Faxen-Irving 2011 & Length of hospital stay (days) & 51 & $10.5($ SD 5.6) & $10.3(\mathrm{SD} 4.9)$ & NS \\
\hline \multirow[t]{2}{*}{ Dennis 2005} & Length of hospital stay (days) & 4023 & 16 (IQR 7-44) & 16 (IQR 7-41) & NS \\
\hline & Median (IQR) & & & & \\
\hline Potter 2001 & $\begin{array}{l}\text { Length of hospital stay (median } \\
\text { (range)) }\end{array}$ & 381 & $16(3-141)$ & $18(2-76)$ & 0.31 \\
\hline Van den Berg 2015 & $\begin{array}{l}\text { Length of hospital stay (median } \\
\text { (range)) }\end{array}$ & 234 & $\begin{array}{l}11: 10(3-63) \\
12: 10(3-27)\end{array}$ & $11(4-71)$ & NR \\
\hline
\end{tabular}


Table 25. Effects of supplementation of meals on hospitalisation, institutionalisation and death from any cause icontinued)'

\section{Supplementation with ONS (in-hospital)}

\begin{tabular}{llllll}
\hline Potter 2001 & Discharge to home & 381 & $131 / 186$ & $127 / 195$ & NS \\
& Discharge to institution & 381 & $31 / 186$ & $33 / 195$ \\
\hline Van den Berg 2015 & Hospital readmissions & 246 & $11: 13$ & 15 & NR \\
& & & $12: 24$ & \\
\hline
\end{tabular}

IQR: interquartile range; NR not reported; NS: not significant; ONS: oral nutritional supplement

Table 26. Effects of home meal delivery systems on nutritional status and death from any cause

\begin{tabular}{llllll}
\hline & Outcome & (N) & Results & Control \\
\cline { 4 - 6 } & & & Intervention & \\
\hline Weight change & & & & \\
\hline Kretser 2003 & Mean change in weight $(\mathrm{kg})$ & 163 & $1.86(5.3)$ & $-1,04(5.2)$ & 0.0062 \\
\hline Mortality & & & & & \\
\hline Kretser 2003 & Mortality & 203 & $3 / 102(3 \%)$ & $9 / 101(9 \%)$ & NR \\
\hline
\end{tabular}

NR: not reported

\section{AP PE N DICES}

\section{Appendix 1. Search strategies (inception to March 2013)}

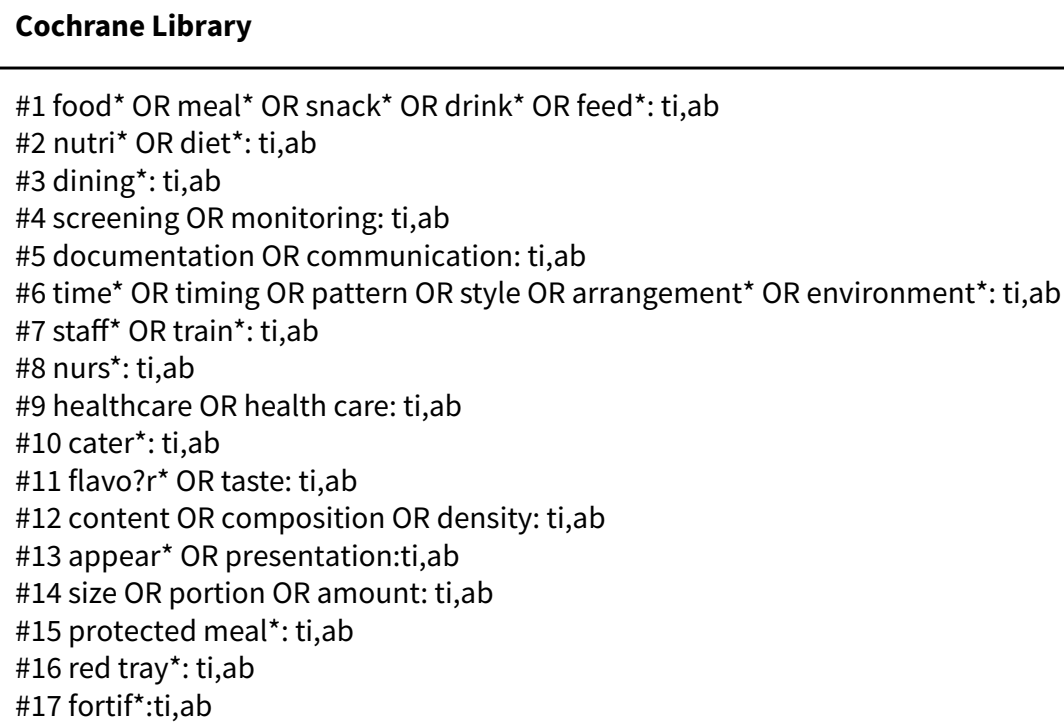


(Continued)

\#18 supplement* ${ }^{*}$ ti,ab

\#19 ((supportive OR nutrition* OR diet ${ }^{\star}$ ) NEAR/3 intervention):ti,ab

\#20 (assist* OR help* OR support*):ti,ab

\#21 (add* OR extra):ti,ab

\#22 (alter* OR chang* OR new OR enhance* OR modif* OR increas* OR decreas* OR improv* OR reduc ${ }^{\star}$ OR target $^{\star}$ ):ti,ab

\#23 (food* OR meal* OR snack* OR drink* OR feed ${ }^{\star}$ ) NEAR/3 ((time* OR timing OR pattern OR style OR arrangement* OR environ-

ment $^{\star}$ ) OR (flavour ${ }^{\star}$ OR flavor ${ }^{\star}$ OR taste) OR (content OR composition OR density) OR (appear ${ }^{\star}$ OR presentation) OR (size OR portion OR amount) OR (fortif*) OR (supplement ${ }^{\star}$ ) OR (assist ${ }^{\star}$ OR help* OR support*) OR (add ${ }^{\star}$ OR extra) OR (alter ${ }^{\star}$ OR chang* OR new OR enhance ${ }^{\star}$ OR modif* OR increas* OR decreas* OR improv* OR reduc* OR target $\left.\left.{ }^{\star}\right)\right)$ :ti,ab \#24 (nutri ${ }^{\star}$ OR diet ${ }^{\star}$ ) NEAR/4 ((content OR composition OR density) OR (fortif*) OR (supplement ${ }^{\star}$ ) OR (add ${ }^{\star}$ OR extra) OR (alter ${ }^{\star}$ OR chang* OR new OR enhance* OR modif* $\quad$ OR increas* OR decreas ${ }^{\star}$ OR improv* OR reduc* OR target $\left.\left.{ }^{\star}\right)\right)$ :ti,ab \#25 dining ${ }^{\star}$ NEAR/4 ((time* OR timing OR pattern OR style OR arrangement* OR environment ${ }^{\star}$ ) OR (alter ${ }^{\star}$ OR chang $^{\star}$ OR new OR enhance* OR modif* OR increas* OR decreas* OR improv* OR reduc* OR target*)):ti, ab

\#26 (screening OR monitoring) NEAR/4 ((nutri ${ }^{\star}$ OR diet ${ }^{\star}$ ) OR (assist ${ }^{\star}$ OR help* OR support ${ }^{\star}$ ) OR (add ${ }^{\star}$ OR extra) OR (alter ${ }^{\star}$ OR chang* OR new OR enhance ${ }^{\star}$ OR modif* OR increas* OR decreas* OR improv* OR reduc ${ }^{\star}$ OR target*)):ti,ab

\#27 (documentation OR communication) NEAR/4 (alter* OR chang* OR new OR enhance* OR modif* OR increas* OR decreas* OR improv $^{\star}$ OR reduc ${ }^{\star}$ OR target $\left.)^{\star}\right)$ ti,ab

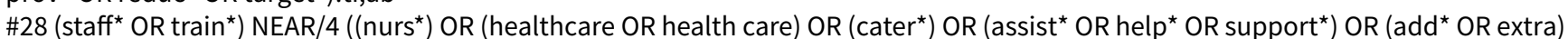
OR (alter ${ }^{\star}$ OR chang ${ }^{\star}$ OR new OR enhance ${ }^{\star}$ OR modif* OR increas* OR decreas* OR improv ${ }^{\star}$ OR reduc ${ }^{\star}$ OR target $\left.\left.{ }^{\star}\right)\right): t i$, ab \#29 supplement* NEAR/5 (add* OR extra):ti,ab

\#30 (assist ${ }^{\star}$ OR help ${ }^{\star}$ OR support ${ }^{\star}$ ) NEAR/4 ((nurs $\left.{ }^{\star}\right)$ OR (healthcare OR health care) OR (cater $\left.\left.{ }^{\star}\right)\right): t i, a b$

$\# 31$ (\#15 OR \#16 OR \#19)

\#32 (\#23 OR \#24 OR \#25 OR \#26 OR \#27 OR \#28 OR \#29 OR \#30 OR \#31)

\#33 (low BMI OR low body mass index):ti,ab

\#34 (low weight OR underweight OR under-weight):ti,ab

\#35 (maln*):ti,ab

\#36 (nutritional risk OR (risk NEAR/4 maln*)):ti,ab

\#37 (poor nutr* OR undernourish* OR under-nourish*):ti,ab

\#38 ((poor OR inadequate OR suboptimal) NEAR/5 intake*):ti,ab

\#39 (institutionali?ed):ti,ab

\#40 (elderly):ti,ab

\#41 (homebound OR home-bound OR housebound OR house-bound):ti,ab

\#42 ((extended OR longterm OR long-term OR community) NEAR/1 care):ti,ab

\#43 ((nursing OR care OR residential) NEAR/1 home):ti,ab

\#44 (inpatient* OR hospitali?* OR hospital patient*):ti,ab

\#45 exp Nutritional Status/

\#46 exp Nutrition Disorders/

\#47 exp Nutrition Assessment/

\#48 exp Nutritional Support/

\#49 exp Nutrition Policy/

\#50 exp Malnutrition/

\#51 diet/

\#52 dietetics/

\#53 hospital food service/

\#54 energy intake/

\#55 fortified food/

\#56 \#33 OR \#34 OR \#35 OR \#36 OR \#37 OR \#38 OR \#39 OR \#40 OR \#41 OR \#42 OR \#43 OR \#44 OR \#45 OR \#46 OR \#47 OR \#48 OR \#49 OR \#50 OR \#51 OR \#52 OR \#53 OR \#54 OR \#55

\#57 32 AND 56

\#58 exp Pregnancy/

\#59 pregnan*:kw,ti

\#60 \#58 OR \#59

\#61 \#57 NOT \#60

\#62 (child OR infant OR paediatric OR pediatric):kw,ti

\#63 \#61 NOT \#62

\#64 (animal OR rat OR mouse OR guinea pig OR primate OR monkey OR cat OR dog):kw,ti

\#65 \#63 NOT \#64

\section{MEDLINE + OLDMEDLINE}


(Continued)

\#1 (food* OR meal* OR snack* OR drink* OR feed*).ab,ti.

\#2 (nutri* OR diet*).ab,ti.

\#3 "dining*".ab,ti.

\#4 (screening OR monitoring).ab,ti.

\#5 (documentation OR communication).ab,ti.

\#6 (time* OR timing OR pattern OR style OR arrangement* OR environment).ab,ti.

\#7 (staff* OR train*).ab,ti.

\#8 "nurs"..ab,ti.

\#9 (healthcare OR health care).ab,ti.

\#10 "cater*".ab,ti.

\#11 (flavo? ${ }^{\star}$ OR taste).ab,ti.

\#12 (content OR composition OR density).ab,ti.

\#13 (appear* OR presentation).ab,ti.

\#14 (size OR portion OR amount).ab,ti.

\#15 "protected meal ${ }^{\star}$.ab,ti.

\#16 "red tray*".ab,ti.

\#17 "fortif*".ab,ti.

\#18 "supplement*".ab,ti.

\#19 ((supportive OR nutrition* OR diet*) ADJ3 intervention).ab,ti.

\#20 (assist* OR help* OR support*).ab,ti.

\#21 (add* OR extra).ab,ti.

\#22 (alter ${ }^{\star}$ OR chang* OR new OR enhance* OR modif* OR increas* OR decreas* OR improv* OR reduc ${ }^{\star}$ OR target $^{\star}$ ).ab,ti.

\#23 1 ADJ3 (6 OR 11 OR 12 OR 13 OR 14 OR 17 OR 18 OR 20 OR 21 OR 22)

\#24 2 ADJ4 (12 OR 17 OR 18 OR 21 OR 22)

\#25 3 ADJ4 (6 OR 22)

\#26 4 ADJ4 (2 OR 21 OR 22)

\#27 5 ADJ4 22

\#28 7 ADJ4 (8 OR 9 OR 10 OR 20 OR 21 OR 22)

\#29 18 ADJ5 21

\#30 20 ADJ4 (8 OR 9 OR 10)

\#31 15 OR 16 OR 19

\#32 23 OR 24 OR 25 OR 26 OR 27 OR 28 OR 29 OR 30 OR 31

\#33 (low bmi OR low body mass index).ab,ti.

\#34 (low weight OR underweight OR under-weight).ab,ti.

\#35 "maln*".ab,ti.

\#36 (nutritional risk OR (risk ADJ4 maln*)).ab,ti.

\#37 (poor nutr* OR undernourish* OR under-nourish*).ab,ti.

\#38 ((poor OR inadequate OR suboptimal) adj5 intake*).ab,ti.

\#39 institutionali?ed.ab,ti.

\#40 elderly.ab,ti.

\#41 (homebound OR home-bound OR housebound OR house-bound).ab,ti.

\#42 ((extended OR longterm OR long-term OR community) ADJ1 care).ab,ti.

\#43 ((nursing OR care OR residential) ADJ1 home).ab,ti.

\#44 (inpatient ${ }^{\star}$ OR hospitali?* OR hospital patient ${ }^{\star}$ ).ab,ti.

\#45 exp Nutritional Status/

\#46 exp Nutrition Disorders/dh, th [Diet Therapy, Therapy]

\#47 nutrition assessment.sh.

\#48 nutritional support.sh.

\#49 nutrition policy.sh.

\#50 exp Malnutrition/dh, th [Diet Therapy, Therapy]

\#51 *diet/

\#52 *dietetics/

\#53 *food service, hospital/

\#54 *energy intake/

$\# 55$ *food, fortified/

\#56 33 OR 34 OR 35 OR 36 OR 37 OR 38 OR 39 OR 40 OR 41 OR 42 OR 43 OR 44 OR 45 OR 46 OR 47 OR 48 OR 49 OR 50 OR 51 OR 52 OR

53 OR 54 OR 55

\#57 32 AND 56

\#58 randomized controlled trial.pt.

\#59 controlled clinical trial.pt. 
(Continued)

\#60 randomi?ed.ab.

\#61 placebo.ab.

\#62 clinical trials as topic.sh.

\#63 randomly.ab.

\#64 trial.ti.

\#65 58 OR 59 OR 60 OR 61 OR 62 OR 63 OR 64

\#66 meta-analysis.pt

\#67 exp technology assessment, biomedical/

\#68 exp meta-analysis/

\#69 exp meta-analysis as topic/

$\# 70$ hta.tw, ot.

\#71 (health technology ADJ6 assessment\$).tw,ot.

\#72 (meta analy\$ OR metaanaly\$ or meta?analy\$).tw,ot.

\#73 ((review\$ OR search\$) ADJ10 (literature\$ OR medical database\$ OR medline OR pubmed OR embase OR cochrane OR cinahl OR

psycinfo OR psyclit OR healthstar OR biosis OR current content\$ OR systemat\$)).tw,ot.

\#74 66 OR 67 OR 68 OR 69 OR 70 OR 71 OR 72 OR 73

\#75 65 OR 74

\#76 (comment OR editorial OR historical-article).pt.

\#77 75 NOT 76

\#78 57 AND 77

\#79 (animals NOT (animals AND humans)).sh.

\#80 78 NOT 79

\#81 exp Pregnancy/

\#82 pregnan*.tw,ot.

\#83 81 OR 82

\#84 80 NOT 83

\#85 limit 84 to "all adult (19 plus years)"

\section{MEDLINE in-process \& other non-indexed citations}

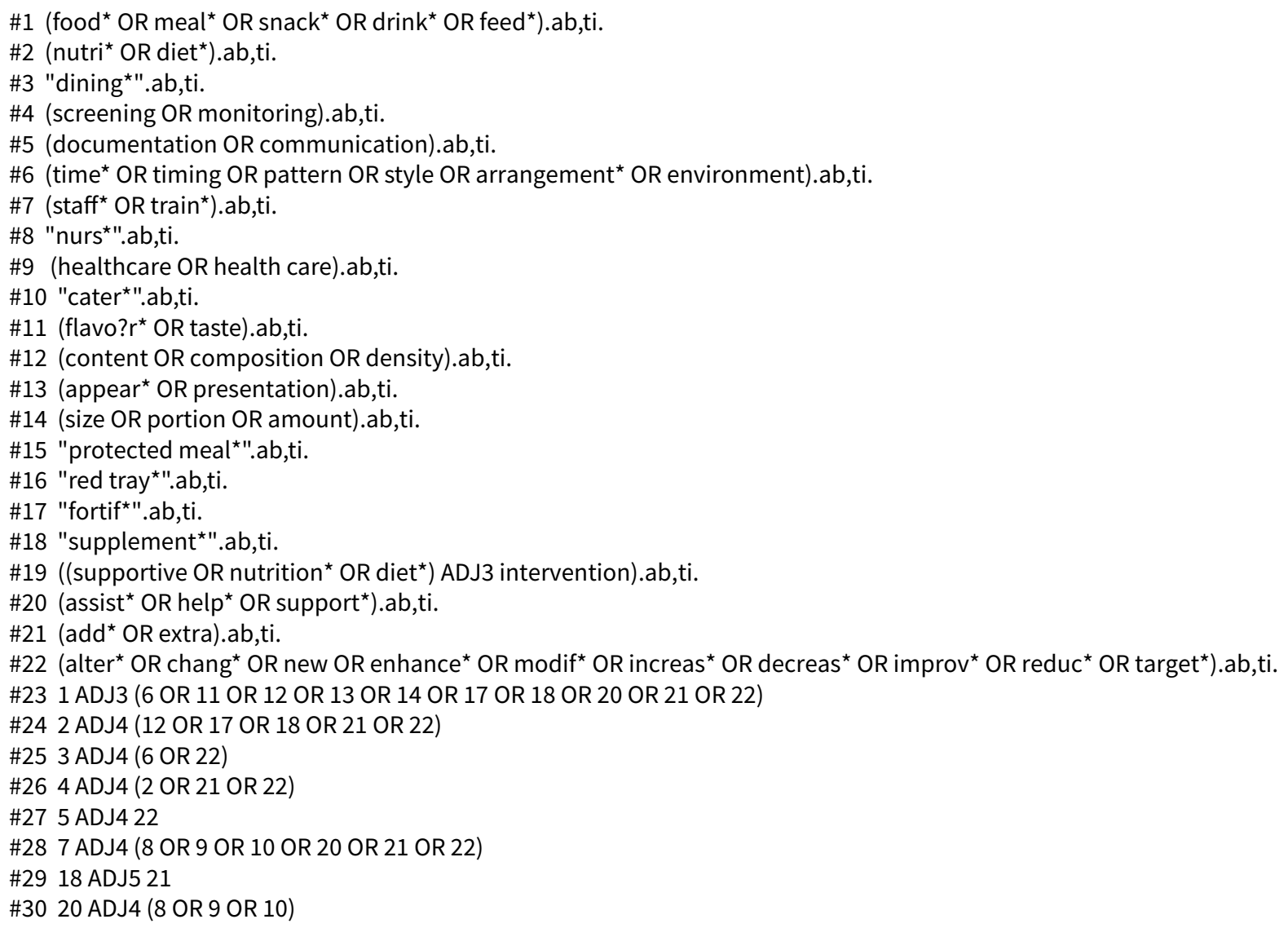


(Continued)

\#31 15 OR 16 OR 19

\#32 23 OR 24 OR 25 OR 26 OR 27 OR 28 OR 29 OR 30 OR 31

\#33 (low bmi OR low body mass index).ab,ti.

\#34 (low weight OR underweight OR under-weight).ab,ti.

\#35 "maln*".ab,ti.

\#36 (nutritional risk OR (risk ADJ4 maln*)).ab,ti.

\#37 (poor nutr ${ }^{\star}$ OR undernourish ${ }^{\star}$ OR under-nourish ${ }^{\star}$ ).ab,ti.

\#38 ((poor OR inadequate OR suboptimal) adj5 intake*).ab,ti.

\#39 institutionali?ed.ab,ti.

\#40 elderly.ab,ti.

\#41 (homebound OR home-bound OR housebound OR house-bound).ab,ti.

\#42 ((extended OR longterm OR long-term OR community) ADJ1 care).ab,ti.

\#43 ((nursing OR care OR residential) ADJ1 home).ab,ti.

\#44 (inpatient ${ }^{\star}$ OR hospitali?* OR hospital patient ${ }^{\star}$ ).ab,ti.

\#45 33 OR 34 OR 35 OR 36 OR 37 OR 38 OR 39 OR 40 OR 41 OR 42 OR 43 OR 44

\#46 32 AND 45

\#47 (random* OR rct*).tw,ot.

\#48 "single blind".tw, ot.

\#49 “double blind".tw, ot.

\#50 ((triple OR treble) AND blind $\left.{ }^{\star}\right)$.tw,ot.

\#51 ((control ${ }^{\star}$ AND trial $\left.{ }^{\star}\right)$ OR (clinical ADJ4 trial $\left.{ }^{\star}\right)$ OR trial $\left.{ }^{\star}\right)$.tw,ot.

\#52 (systematic ${ }^{\star}$ review $^{\star}$ ).tw,ot.

\#53 hta.tw, ot.

\#54 (health technology ADJ6 assessment\$).tw,ot.

\#55 (meta analy\$ OR metaanaly\$ or meta?analy\$).tw,ot.

\#56 ((review\$ OR search\$) ADJ10 (literature\$ OR medical database\$ OR medline OR pubmed OR embase OR cochrane OR cinahl OR

psycinfo OR psyclit OR healthstar OR biosis OR current content\$ OR systemat\$)).tw,ot.

\#57 47 OR 48 OR 49 OR 50 OR 51 OR 52 OR 53 OR 54 OR 55 OR 56

\#58 (comment OR editorial OR historical-article).pt.

\#59 57 NOT 58

\#60 46 AND 59

\#61 pregnan*.tw,ot.

\#62 60 NOT 61

\section{Embase + Embase classic}

\#1 ( food $^{\star}$ OR meal ${ }^{\star}$ OR snack ${ }^{\star}$ OR drink ${ }^{\star}$ OR feed $\left.^{\star}\right)$.ab,ti.

\#2 (nutri* OR diet ${ }^{\star}$ ).ab,ti.

\#3 "dining*".ab,ti.

\#4 (screening OR monitoring).ab,ti.

\#5 (documentation OR communication).ab,ti.

\#6 (time* OR timing OR pattern OR style OR arrangement* OR environment).ab,ti.

\#7 ( staff $^{\star}$ OR train $\left.{ }^{\star}\right) \cdot$ ab,ti.

\#8 "nurs*".ab,ti.

\#9 (healthcare OR health care).ab,ti.

\#10 "cater*".ab,ti.

\#11 (flavo? $r^{\star}$ OR taste).ab,ti.

\#12 (content OR composition OR density).ab,ti.

\#13 (appear* OR presentation).ab,ti.

\#14 (size OR portion OR amount).ab,ti.

\#15 "protected meal ${ }^{\star}$.ab,ti.

\#16 "red tray*".ab,ti.

\#17 "fortif*".ab,ti.

\#18 "supplement*".ab,ti.

\#19 ((supportive OR nutrition* OR diet*) ADJ3 intervention).ab,ti.

\#20 (assist* OR help* OR support ${ }^{\star}$ ).ab,ti.

\#21 (add* OR extra).ab,ti.

\#22 (alter ${ }^{\star}$ OR chang* OR new OR enhance* OR modif* OR increas* OR decreas* OR improv* OR reduc* OR target*).ab,ti.

\#23 1 ADJ3 (6 OR 11 OR 12 OR 13 OR 14 OR 17 OR 18 OR 20 OR 21 OR 22)

\#24 2 ADJ4 (12 OR 17 OR 18 OR 21 OR 22) 
(Continued)

\#25 3 ADJ4 (6 OR 22)

\#26 4 ADJ4 (2 OR 21 OR 22)

\#27 5 ADJ4 22

\#28 7 ADJ4 (8 OR 9 OR 10 OR 20 OR 21 OR 22)

\#29 18 ADJ5 21

\#30 20 ADJ4 (8 OR 9 OR 10)

\#31 15 OR 16 OR 19

\#32 23 OR 24 OR 25 OR 26 OR 27 OR 28 OR 29 OR 30 OR 31

\#33 (low bmi OR low body mass index).ab,ti.

\#34 (low weight OR underweight OR under-weight).ab,ti.

\#35 "maln*".ab,ti.

\#36 (nutritional risk OR (risk ADJ4 maln*)).ab,ti.

\#37 (poor nutr* OR undernourish* OR under-nourish*).ab,ti.

\#38 ((poor OR inadequate OR suboptimal) adj5 intake*).ab,ti.

\#39 institutionali?ed.ab,ti.

\#40 elderly.ab,ti.

\#41 (homebound OR home-bound OR housebound OR house-bound).ab,ti.

\#42 ((extended OR longterm OR long-term OR community) ADJ1 care).ab,ti.

\#43 ((nursing OR care OR residential) ADJ1 home).ab,ti.

\#44 (inpatient ${ }^{\star}$ OR hospitali?* OR hospital patient ${ }^{\star}$ ).ab,ti.

\#45 exp Nutritional Status/

\#46 exp Nutritional Disorder/dh, th [Therapy]

\#47 nutrition assessment.sh.

\#48 nutritional support.sh.

\#49 health care policy.sh.

\#50 exp Malnutrition/dh, th [Therapy]

\#51 *diet/

\#52 *dietetics/

\#53 * food service, hospital/

\#54 *energy intake/

\#55 *food, fortified/

\#56 33 OR 34 OR 35 OR 36 OR 37 OR 38 OR 39 OR 40 OR 41 OR 42 OR 43 OR 44 OR 45 OR 46 OR 47 OR 48 OR 49 OR 50 OR 51 OR 52 OR

53 OR 54 OR 55

\#57 32 AND 56

\#58 (random* OR rct $\left.{ }^{\star}\right)$.tw,ot.

\#59 “single blind"..tw, ot.

\#60 “double blind"”.tw, ot.

\#61 ((triple OR treble) AND blind $\left.{ }^{\star}\right)$.tw,ot.

\#62 ((control* AND trial $\left.{ }^{\star}\right)$ OR (clinical ADJ4 trial $\left.^{\star}\right)$ OR trial $\left.{ }^{\star}\right)$.tw,ot.

\#63 (systematic ${ }^{\star}$ review $^{\star}$ ).tw,ot.

\#64 hta.tw, ot.

\#65 (health technology ADJ6 assessment\$).tw,ot.

\#66 (meta analy\$ OR metaanaly\$ or meta?analy\$).tw,ot.

\#67 ((review\$ OR search\$) ADJ10 (literature\$ OR medical database\$ OR medline OR pubmed OR embase OR cochrane OR cinahl OR psycinfo OR psyclit OR healthstar OR biosis OR current content\$ OR systemat\$)).tw,ot.

\#68 58 OR 59 OR 60 OR 61 OR 62 OR 63 OR 64 OR 65 OR 66 OR 67

\#69 (comment OR editorial OR historical-article).pt.

\#70 68 NOT 69

\#71 57 AND 70

\#72 exp Pregnancy/

\#73 pregnan*.tw,ot.

\#74 72 OR 73

\#75 71 NOT 74

\#76 limit 75 to (human and (adult $<18$ to 64 years $>$ or aged $<65+$ years $>$ ))

\section{AMED}

$\# 1$ (food* OR meal* OR snack* OR drink* OR feed*).ti,ab

\#2 (nutri* OR diet*).ti,ab

\#3 "dining*".ti,ab 
(Continued)

\#4 screening OR monitoring).ti,ab

\#5 documentation OR communication).ti,ab

\#6 time* OR timing OR pattern OR style OR arrangement* OR environment).ti,ab

\#7 staff* OR train $\left.{ }^{\star}\right) . t i, a b$

\#8 nurs ${ }^{\star}$.ti,ab

\#9 healthcare OR “health care").ti,ab

\#10 cater*".ti,ab

\#11 flavo?r* OR taste).ti,ab

\#12 content OR composition OR density).ti,ab

\#13 appear* OR presentation).ti,ab

\#14 size OR portion OR amount).ti,ab

\#15 protected meal*".ti,ab

\#16 red tray ".ti,ab

\#17 fortif*".ti,ab

\#18 "supplement*".ti,ab

\#19 ((supportive OR nutrition* OR diet ${ }^{\star}$ ) ADJ3 intervention).ti,ab

\#20 (assist* OR help* OR support ${ }^{\star}$ ).ti,ab

\#21 (add* OR extra).ti,ab

\#22 alter* OR chang* OR new OR enhance* OR modif* OR increas* OR decreas* OR improv* OR reduc* OR target*).ti,ab

\#23 1 DJ3 (6 OR 11 OR 12 OR 13 OR 14 OR 17 OR 18 OR 20 OR 21 OR 22)

\#24 2 ADJ4 (12 OR 17 OR 18 OR 21 OR 22)

\#25 3 ADJ4 (6 OR 22)

\#26 4 ADJ4 (2 OR 21 OR 22)

\#27 5 ADJ4 22

\#28 7 ADJ4 (8 OR 9 OR 10 OR 20 OR 21 OR 22)

\#29 18 ADJ5 21

\#30 20 ADJ4 (8 OR 9 OR 10)

\#31 15 OR 16 OR 19

\#32 23 OR 24 OR 25 OR 26 OR 27 OR 28 OR 29 OR 30 OR 31

\#33 ("low bmi" OR "low body mass index").ti,ab

\#34 ("low weight" OR underweight OR under-weight).ti,ab

\#35 "maln*".ti,ab

\#36 ("nutritional risk" OR (risk ADJ4 maln*)).ti,ab

\#37 ("poor nutr*” OR undernourish* OR under-nourish*).ti,ab

\#38 ((poor OR inadequate OR suboptimal) ADJ5 intake*).ti,ab

\#39 institutionali?ed.ti,ab

\#40 elderly.ti,ab

\#41 (homebound OR home-bound OR housebound OR house-bound).ti,ab

\#42 ((extended OR longterm OR long-term OR community) ADJ1 care).ti,ab

\#43 ((nursing OR care OR residential) ADJ1 home).ti,ab

\#44 (inpatient* OR hospitali?* OR hospital patient ${ }^{\star}$ ).ti,ab

\#45 33 OR 34 OR 35 OR 36 OR 37 OR 38 OR 39 OR 40 OR 41 OR 42 OR 43 OR 44

\#46 2 AND 45

\#47 (random* OR rct*).ti,ab

\#48 “single blind*".ti,ab

\#49 “double blind*”.ti,ab

\#50 ((triple OR treble) AND blind $\left.{ }^{\star}\right)$.ti,ab

\#51 ((control ${ }^{\star}$ AND trial ${ }^{\star}$ ) OR (clinical ADJ4 trial ${ }^{\star}$ ) OR trial*).ti,ab

\#52 (systematic* review $^{\star}$ ).ti,ab

\#53 ("health technology" ADJ6 assessment\$).ti,ab

\#54 hta.ti,ab

\#55 ("meta analy\$” OR metaanaly\$ or meta?analy\$).ti,ab

\#56 47 OR 48 OR 50 OR 51 OR 52 OR 53 OR 54 OR 55

\#57 46 AND 56

\#58 (comment OR editorial OR historical-article).pt.

\#59 57 NOT 58

\#60 (animal OR rat OR mouse OR guinea pig OR primate OR monkey OR cat OR dog).ti,ab

\#61 9 NOT 60

\#62 regnan*.ti,ab

\#63 61 NOT 62

\#64 (child* OR infant OR paediatric OR pediatric).ti,ab 


\section{British Nursing Index}

\#1 (food ${ }^{\star}$ OR meal ${ }^{\star}$ OR snack ${ }^{\star}$ OR drink ${ }^{\star}$ OR feed $\left.{ }^{\star}\right)$.ab,ti.

\#2 (nutri* OR diet ${ }^{\star}$ ).ab,ti.

\#3 "dining*".ab,ti.

\#4 (screening OR monitoring).ab,ti.

\#5 (documentation OR communication).ab,ti.

\#6 (time* OR timing OR pattern OR style OR arrangement ${ }^{\star}$ OR environment).ab,ti.

\#7 (staff* OR train*).ab,ti.

\#8 "nurs*".ab,ti.

\#9 (healthcare OR health care).ab,ti.

\#10 "cater*".ab,ti.

\#11 (flavo? r* OR taste).ab,ti.

\#12 (content OR composition OR density).ab,ti.

\#13 (appear* OR presentation).ab,ti.

\#14 (size OR portion OR amount).ab,ti.

\#15 "protected meal*".ab,ti.

\#16 "red tray*".ab,ti.

\#17 "fortif*".ab,ti.

\#18 "supplement*".ab,ti.

\#19 ((supportive OR nutrition* OR diet*) ADJ3 intervention).ab,ti.

\#20 (assist* OR help* OR support ${ }^{\star}$ ).ab,ti.

\#21 (add* OR extra).ab,ti.

\#22 (alter* OR chang* OR new OR enhance* OR modif* OR increas* OR decreas* OR improv* OR reduc ${ }^{\star}$ OR target $^{\star}$ ). ab,ti.

\#23 1 ADJ3 (6 OR 11 OR 12 OR 13 OR 14 OR 17 OR 18 OR 20 OR 21 OR 22)

\#24 2 ADJ4 (12 OR 17 OR 18 OR 21 OR 22)

\#25 3 ADJ4 (6 OR 22)

\#26 4 ADJ4 (2 OR 21 OR 22)

\#27 5 ADJ4 22

\#28 7 ADJ4 (8 OR 9 OR 10 OR 20 OR 21 OR 22)

\#29 18 ADJ5 21

\#30 20 ADJ4 (8 OR 9 OR 10)

\#31 15 OR 16 OR 19

\#32 23 OR 24 OR 25 OR 26 OR 27 OR 28 OR 29 OR 30 OR 31

\#33 (low bmi OR low body mass index).ab,ti.

\#34 (low weight OR underweight OR under-weight).ab,ti.

\#35 "maln*".ab,ti.

\#36 (nutritional risk OR (risk ADJ4 maln*)).ab,ti.

\#37 (poor nutr* OR undernourish* OR under-nourish ${ }^{\star}$ ).ab,ti.

\#38 ((poor OR inadequate OR suboptimal) adj5 intake*).ab,ti.

\#39 institutionali?ed.ab,ti.

\#40 elderly.ab,ti.

\#41 homebound OR home-bound OR housebound OR house-bound).ab,ti.

\#42 ((extended OR longterm OR long-term OR community) ADJ1 care).ab,ti.

\#43 ((nursing OR care OR residential) ADJ1 home).ab,ti.

\#44 (inpatient* OR hospitali?* OR hospital patient ${ }^{\star}$ ).ab,ti.

\#45 33 OR 34 OR 35 OR 36 OR 37 OR 38 OR 39 OR 40 OR 41 OR 42 OR 43 OR 44

\#46 32 AND 45

\#47 (random* OR rct*).tw,ot.

\#48 "single blind*”.tw, ot.

\#49 “double blind".tw, ot.

\#50 ((triple OR treble) AND blind*).tw,ot.

\#51 ((control ${ }^{\star}$ AND trial $\left.{ }^{\star}\right)$ OR (clinical ADJ4 trial $\left.{ }^{\star}\right)$ OR trial $\left.{ }^{\star}\right)$.tw,ot.

\#52 (systematic ${ }^{\star}$ review*).tw,ot.

\#53 hta.tw, ot.

\#54 (health technology ADJ6 assessment\$).tw,ot.

\#55 (meta analy\$ OR metaanaly\$ or meta?analy\$).tw,ot. 
(Continued)

\#56 (review\$ OR search\$) ADJ10 (literature\$ OR medical database\$ OR medline OR pubmed OR embase OR cochrane OR cinahl OR psycinfo OR psyclit OR healthstar OR biosis OR current content\$ OR systemat\$)).tw,ot. \#57 47 OR 48 OR 49 OR 50 OR 51 OR 52 OR 53 OR 54 OR 55 OR 56

\#58 (comment or editorial or historical-article).pt.

\#59 57 NOT 58

\#60 46 AND 59

\#61 pregnan ${ }^{\star}$.tw,ot.

\#62 60 NOT 61

\section{CINAHL}

\#1 (TI (food ${ }^{\star}$ OR meal ${ }^{\star}$ OR snack ${ }^{\star}$ OR drink ${ }^{\star}$ OR feed $\left.\left.{ }^{\star}\right)\right)$ OR (AB (food ${ }^{\star}$ OR meal ${ }^{\star}$ OR snack ${ }^{\star}$ OR drink ${ }^{\star}$ OR feed $\left.\left.{ }^{\star}\right)\right)$

\#2 (TI (nutri* OR $\left.\left.\operatorname{diet}^{\star}\right)\right)$ OR (AB (nutri* OR $\left.\left.\operatorname{diet}^{\star}\right)\right)$

\#3 (TI dining $\left.{ }^{\star}\right)$ OR (AB dining $\left.{ }^{\star}\right)$

\#4 ( $\mathrm{TI}$ (screening OR monitoring)) OR (AB (screening OR monitoring))

\#5 ( $\mathrm{TI}$ (documentation OR communication)) OR (AB (documentation OR communication))

\#6 (TI (time* OR timing OR pattern OR style OR arrangement* OR environment $\left.{ }^{\star}\right)$ ) OR (AB (time* OR timing OR pattern OR style OR arrangement* $\mathrm{OR}$ environment $\left.\left.\mathrm{C}^{\star}\right)\right)$

\#7 (TI (staff* OR train*)) OR (AB (staff* OR train*))

\#8 (TI nurs $\left.{ }^{\star}\right)$ OR (AB nurs $\left.{ }^{\star}\right)$

\#9 (TI (healthcare OR health care)) OR (AB (healthcare OR health care))

$\# 10$ (TI cater $\left.{ }^{\star}\right)$ OR (AB cater $)$

\#11 (TI (flavo? $r^{\star}$ OR taste)) OR (AB (flavo? ${ }^{\star}$ OR taste))

\#12 (TI (content OR composition OR density)) OR (AB (content OR composition OR density))

\#13 (TI (appear* OR presentation)) OR (AB (appear* OR presentation))

\#14 (TI (size OR portion OR amount)) OR (AB (size OR portion OR amount))

$\# 15$ (TI (protected meal $\left.\left.{ }^{*}\right)\right)$ OR (AB (protected meal $\left.\left.{ }^{\star}\right)\right)$

$\# 16\left(\mathrm{TI}\left(\right.\right.$ red tray $\left.\left.^{\star}\right)\right)$ OR $\left(\mathrm{AB}\left(\right.\right.$ protected meal $\left.\left.{ }^{\star}\right)\right)$

$\# 17$ (TI fortif*) OR (AB fortif*)

$\# 18$ (TI supplement $\left.{ }^{\star}\right)$ OR (AB supplement*)

\#19 (TI ((supportive OR nutrition* OR diet*) N3 intervention)) OR (AB ((supportive OR nutrition* OR diet*) N3 intervention))

\#20 (TI (assist* OR help* OR support* $\left.{ }^{\star}\right)$ OR (AB (assist* OR help* OR support*))

$\# 21$ (TI (add* OR extra)) OR (AB (add* OR extra))

\#22 (TI (alter ${ }^{\star}$ OR chang* OR new OR enhance* OR modif* OR increas* OR decreas* OR improv* OR reduc* OR target $\left.{ }^{\star}\right)$ ) OR (AB (alter OR chang* OR new OR enhance* OR modif* OR increas* OR decreas ${ }^{\star}$ OR improv* OR reduc ${ }^{\star}$ OR target Or $\left.^{\star}\right)$

\#23 (TI (food* OR meal* OR snack* OR drink* OR feed ${ }^{\star}$ ) N3 ((time* OR timing OR pattern OR style OR arrangement* OR environment ${ }^{\star}$ ) OR (flavour ${ }^{\star}$ OR flavor ${ }^{\star}$ OR taste) OR (content OR composition OR density) OR (appear ${ }^{\star}$ OR presentation) OR (size OR portion OR amount) OR (fortif*) OR (supplement ${ }^{\star}$ ) OR (assist* OR help* OR support ${ }^{\star}$ ) OR (add ${ }^{\star}$ OR extra) OR (alter ${ }^{\star}$ OR chang* OR new OR enhance ${ }^{\star}$ OR modif* OR increas ${ }^{\star}$ OR decreas ${ }^{\star}$ OR improv* OR reduc $^{\star}$ OR target $\left.\left.{ }^{\star}\right)\right)$ ) OR (AB (food ${ }^{\star}$ OR meal ${ }^{\star}$ OR snack ${ }^{\star}$ OR drink ${ }^{\star}$ OR feed $^{\star}$ ) N3 ((time* OR timing OR pattern OR style OR arrangement* OR environment ${ }^{\star}$ ) OR (flavour ${ }^{\star}$ OR flavor ${ }^{\star}$ OR taste) OR (content OR composition OR density) OR (appear* OR presentation) OR (size OR portion OR amount) OR (fortif*) OR (supplement ${ }^{\star}$ ) OR (assist* OR help ${ }^{\star}$ OR support ${ }^{\star}$ ) OR (add* OR extra) OR (alter* OR chang* OR new OR enhance* OR modif* OR increas* OR decreas* OR improv* OR reduc* OR target $\left.\left.{ }^{\star}\right)\right)$ )

\#24 (TI (nutri* OR diet*) N4 ((content OR composition OR density) OR (fortif*) OR (supplement*) OR (add* OR extra) OR (alter* OR chang* OR new OR enhance* OR modif* OR increas* OR decreas* OR improv* OR reduc ${ }^{\star}$ OR target in $\left.^{\star}\right)$ ) OR (AB (nutri ${ }^{\star}$ OR diet*) N4

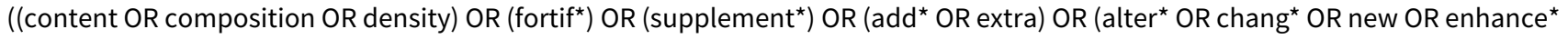
OR modif* OR increas* OR decreas* OR improv* OR reduc ${ }^{\star}$ OR target $\left.\left.{ }^{\star}\right)\right)$ )

\#25 (TI dining* N4 ((time* OR timing OR pattern OR style OR arrangement* OR environment ${ }^{\star}$ ) OR (alter ${ }^{\star}$ OR chang $^{\star}$ OR new OR enhance* OR modif* OR increas* OR decreas* OR improv* OR reduc* OR target*))) OR (AB dining* N4 ((time* OR timing OR pattern OR style OR arrangement ${ }^{\star}$ OR environment ${ }^{\star}$ ) OR (alter ${ }^{\star}$ OR chang ${ }^{\star}$ OR new OR enhance* OR modif* OR increas ${ }^{\star}$ OR decreas $^{\star}$ OR improv $^{\star}$ OR reduc* OR target $\left.\left.{ }^{\star}\right)\right)$ )

\#26 (TI (screening OR monitoring) N4 ((nutri* OR diet*) OR (assist* OR help* OR support*) OR (add* OR extra) OR (alter ${ }^{\star}$ OR chang* OR new OR enhance OR modif $^{\star}$ OR increas* OR decreas ${ }^{\star}$ OR improv* OR reduc $^{\star}$ OR target $\left.\left.{ }^{\star}\right)\right)$ ) OR (AB (screening OR monitoring) N4 ((nutri $^{\star}$ OR diet ${ }^{\star}$ ) OR (assist ${ }^{\star}$ OR help* OR support ${ }^{\star}$ ) OR (add* OR extra) OR (alter ${ }^{\star}$ OR chang ${ }^{\star}$ OR new OR enhance* OR modif* OR increas ${ }^{\star}$ OR decreas* OR improv* OR reduc* OR target $\left.\left.{ }^{\star}\right)\right)$ )

\#27 (TI (documentation OR communication) N4 ((alter ${ }^{\star}$ OR chang* OR new OR enhance* OR modif* OR increas* OR decreas* OR improv $^{\star}$ OR reduc OR target $\left.^{\star}\right)$ )) OR (AB (documentation OR communication) N4 ((alter* OR chang* OR new OR enhance* OR modif* OR increas* OR decreas* OR improv* OR reduc* OR target $\left.\left.{ }^{\star}\right)\right)$ )

\#28 (TI (staff* OR train ${ }^{\star}$ ) N4 ((nurs $\left.{ }^{\star}\right)$ OR (healthcare OR health care) OR (cater $\left.{ }^{\star}\right)$ OR (assist* OR help* OR support ${ }^{\star}$ ) OR (add ${ }^{\star}$ OR ex-

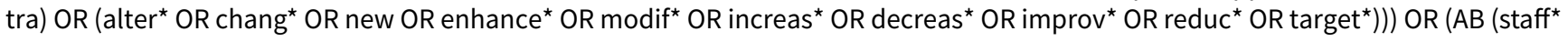


(Continued)

OR train*) N4 ((nurs ${ }^{\star}$ ) OR (healthcare OR health care) OR (cater ${ }^{\star}$ ) OR (assist* OR help* OR support*) OR (add ${ }^{\star}$ OR extra) OR (alter* OR chang* OR new OR enhance* OR modif* OR increas* OR decreas* OR improv* OR reduc* OR target $\left.\left.{ }^{\star}\right)\right)$ )

\#29 (TI (supplement ${ }^{\star}$ N5 (add ${ }^{\star}$ OR extra))) OR (AB (supplement ${ }^{\star}$ N5 (add ${ }^{\star}$ OR extra)))

\#30 (TI (assist* OR help ${ }^{\star}$ OR support $\left.{ }^{\star}\right)$ N4 ((nurs ${ }^{\star}$ ) OR (healthcare OR health care) OR (cater $\left.\left.{ }^{\star}\right)\right)$ ) OR (AB (assist* OR help* OR support ${ }^{\star}$ )

N4 ((nurs $\left.{ }^{\star}\right)$ OR (healthcare OR health care) OR (cater $\left.\left.{ }^{\star}\right)\right)$ )

\#31 (S15 OR S16 OR S19)

\#32 S23 OR S24 OR S25 OR S26 OR S27 OR S28 OR S29 OR S30 OR S31

\#33 (TI (low BMI OR low body mass index)) OR (AB (low BMI OR low body mass index))

\#34 (TI (low weight OR underweight OR under-weight)) OR (AB (low weight OR underweight OR under-weight))

\#35 (TI maln*) OR (AB maln*)

\#36 (TI (nutritional risk OR (risk N4 maln*))) OR (AB (nutritional risk OR (risk N4 maln*)))

\#37 (TI (poor nutr* OR undernourish* OR under-nourish*)) OR (AB (poor nutr* OR undernourish ${ }^{\star}$ OR under-nourish $\left.{ }^{\star}\right)$ )

\#38 TI (poor OR inadequate OR suboptimal) N5 intake*) OR (AB (poor OR inadequate OR suboptimal) N5 intake*)

\#39 (TI (institutionali?ed)) OR (AB (institutionali?ed))

\#40 (TI elderly) OR (AB elderly)

\#41 (TI (homebound OR home-bound OR housebound OR house-bound)) OR (AB (homebound OR home-bound OR housebound OR house-bound))

\#42 (TI ((extended OR longterm OR long-term OR community) N1 care)) OR (AB ((extended OR longterm OR long-term OR community) N1 care))

\#43 (TI ((nursing OR care OR residential) N1 home)) OR (AB ((nursing OR care OR residential) N1 home))

\#44 (TI (inpatient* OR hospitali?* OR hospital patient*)) OR (AB (inpatient* OR hospitali?* OR hospital patient*))

\#45 SU Nutritional Status

\#46 SU Nutrition Disorders

\#47 SU Nutritional Assessment

\#48 SU Nutritional Support

\#49 SU Nutrition Policy

\#50 SU Malnutrition

\#51 SU Diet

\#52 SU Dietetics

\#53 SU Hospital Food Service

\#54 SU Energy Intake

\#55 SU Fortified Food

\#56 S33 OR S34 OR S35 OR S36 OR S37 OR S38 OR S39 OR S40 OR S41 OR S42 OR S43 OR S44 OR S45 OR S46 OR S47 OR S48 OR S49 OR S50 OR S51 OR S52 OR S53 OR S54 OR S55

\#57 S32 AND S56

\#58 (TI (random* OR rct*)) OR (TX (random* OR rct*))

\#59 (TI single blind $\left.{ }^{\star}\right)$ OR (TX single blind $\left.{ }^{\star}\right)$

$\# 60$ (TI double blind ${ }^{\star}$ ) OR (TX double blind $\left.{ }^{\star}\right)$

\#61 (TI ((triple OR treble) AND blind $\left.\left.{ }^{*}\right)\right)$ OR (TX ((triple OR treble) AND blind*))

\#62 (TI ((control ${ }^{*}$ AND trial $\left.{ }^{\star}\right)$ OR (clinical N4 trial $\left.^{\star}\right)$ OR trial $\left.\left.{ }^{\star}\right)\right)$ OR (TX ((control* AND trial $\left.{ }^{\star}\right)$ OR (clinical N4 trial $\left.{ }^{\star}\right)$ OR trial $\left.\left.{ }^{\star}\right)\right)$

\#63 (TI systematic* review*) OR (TX systematic* review*)

\#64 (TI hta) OR (TX hta)

\#65 (TI (health technology N6 assessment*)) OR (TX (health technology N6 assessment $\left.{ }^{\star}\right)$ )

\#66 (TI (meta analy* OR metaanaly* or meta?analy*)) OR (TX (meta analy* OR metaanaly* or meta?analy*))

\#67 (TI ((review* OR search*) N10 (literature* OR medical database* OR medline OR pubmed OR embase OR cochrane OR cinahl OR psycinfo OR psyclit OR healthstar OR biosis OR current content* OR systemat $\left.\left.{ }^{\star}\right)\right)$ ) OR (TX ((review* OR search $)$ N10 (literature* OR medical database* OR medline OR pubmed OR embase OR cochrane OR cinahl OR psycinfo OR psyclit OR healthstar OR biosis OR current content ${ }^{\star}$ OR systemat $\left.\left.{ }^{\star}\right)\right)$ )

\#68 S58 OR S59 OR S60 OR S61 OR S62 OR S63 OR S64 OR S65 OR S66 OR S67

\#69 PT (comment OR editorial OR historical-article)

\#70 S68 NOT S69

\#71 SU Pregnancy

\#72 (TI pregnan*) OR (TX pregnan*)

\#73 S71 OR S72

\#74 S70 NOT S73

\#75 S57 AND S74

Limiters - Human; Age Groups: Adult: 19-44 years, Aged: 65+ years

\section{SCOPUS}


(Continued)

$\# 1$

((TITLE-ABS-KEY ((food ${ }^{*}$ W/3 time ${ }^{\star}$ ) OR (food ${ }^{*}$ W/3 timing) OR (food* W/3 pattern) OR (food ${ }^{*}$ W/3 style) OR (food* W/3 arrangemen$t^{\star}$ )OR (food ${ }^{\star} W / 3$ environment) OR (food ${ }^{\star} W / 3$ flavour) OR (food ${ }^{\star} W / 3$ taste) OR (food ${ }^{\star} W / 3$ content) OR (food* W/3 composition) OR (food ${ }^{\star} W / 3$ density) OR (food ${ }^{\star} W / 3$ appear $^{\star}$ ) OR (food ${ }^{\star} W / 3$ presentation) OR (food ${ }^{\star} W / 3$ size) OR (food $W / 3$ portion) OR (food* W/3 amount) OR (food ${ }^{\star} W / 3$ fortifi*) OR (food ${ }^{\star} W / 3$ supplement $^{\star}$ ) OR (food ${ }^{\star} W / 3$ assist $^{\star}$ ) OR (food $W / 3$ help*) OR (food f $^{\star} / 3$ support $^{\star}$ )

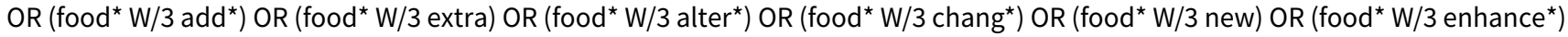
OR (food ${ }^{\star}$ W/3 modif*) OR (food* W/3 increas*) OR (food W/3 decreas*) OR (food* W/3 improv*) OR (food* W/3 reduc ${ }^{\star}$ ) OR (food* W/3 target $\left.\left.\left.{ }^{\star}\right)\right)\right)$ OR (TITLE-ABS-KEY ((meal ${ }^{\star} W / 3$ time $\left.^{\star}\right)$ OR (meal ${ }^{*} W / 3$ timing) OR (meal ${ }^{*}$ W/3 pattern) OR (meal ${ }^{\star} W / 3$ style) OR (meal ${ }^{\star} W / 3$ arrangement $\left.{ }^{\star}\right) O R$ (meal ${ }^{*} W / 3$ environment) OR (meal ${ }^{*} W / 3$ flavour) OR (meal ${ }^{*} W / 3$ taste) OR (meal ${ }^{*} W / 3$ content) OR (meal ${ }^{*} W / 3$ composition) OR (meal ${ }^{\star} W / 3$ density) OR (meal ${ }^{\star} W / 3$ appear $\left.^{\star}\right)$ OR (meal ${ }^{\star} W / 3$ presentation) OR (meal ${ }^{\star} W / 3$ size) OR (meal ${ }^{\star} W / 3$ portion) OR (meal ${ }^{\star} W / 3$ amount) OR (meal ${ }^{\star} W / 3$ fortifi ${ }^{\star}$ ) OR (meal ${ }^{\star} W / 3$ supplement ${ }^{\star}$ ) OR (meal ${ }^{\star} W / 3$ assist ${ }^{\star}$ ) OR (meal ${ }^{\star} W / 3$ help OR $^{\star}$ OReal ${ }^{\star}$

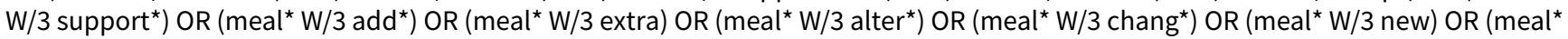

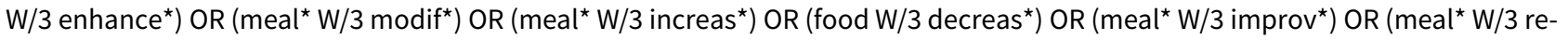
duc $^{\star}$ ) OR (meal ${ }^{\star}$ W/3 target $\left.\left.{ }^{\star}\right)\right)$ ) OR (TITLE-ABS-KEY ((snack* W/3 time* ${ }^{\star}$ ) OR (snack* W/3 timing) OR (snack ${ }^{\star}$ W/3 pattern) OR (snack* W/3 style) OR (snack* W/3 arrangement $\left.{ }^{\star}\right)$ OR (snack*W/3 environment) OR (snack* W/3 flavour) OR (snack* W/3 taste) OR (snack* W/3 content) OR (snack* W/3 composition) OR (snack ${ }^{\star}$ W/3 density) OR (snack* W/3 appear ${ }^{\star}$ ) OR (snack* W/3 presentation) OR (snack* W/3

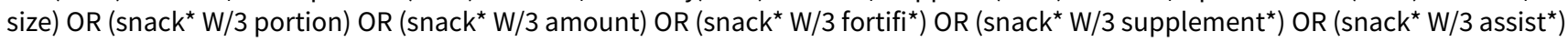
OR (snack* W/3 help*) OR (snack* W/3 support*) OR (snack* W/3 add*) OR (snack* W/3 extra) OR (snack* W/3 alter*) OR (snack* W/3

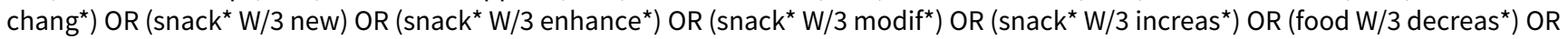

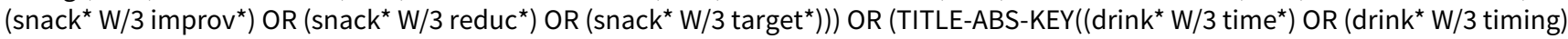
OR (drink ${ }^{*}$ W/3 pattern) OR (drink ${ }^{\star} W / 3$ style) OR (drink* W/3 arrangement ${ }^{\star}$ )OR (drink*W/3 environment) OR (drink ${ }^{\star} W / 3$ flavour) OR (drink* W/3 taste) OR (drink* W/3 content) OR (drink* W/3 composition) OR (drink* W/3 density) OR (drink* W/3 appear*) OR (drink ${ }^{\star}$ W/3 presentation) OR (drink* W/3 size) OR (drink* W/3 portion) OR (drink* W/3 amount) OR (drink ${ }^{*}$ W/3 fortifi*) OR (drink* W/3 supple-

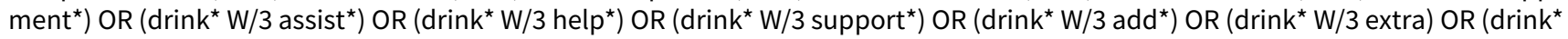

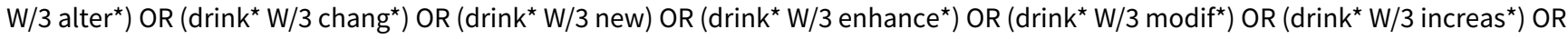
(food W/3 decreas ${ }^{\star}$ ) OR (drink* W/3 improv*) OR (drink* W/3 reduc*) OR (drink* W/3 target*))) OR (TITLE-ABS-KEY((feed ${ }^{\star}$ W/3 time* $)$ OR (feed ${ }^{\star} W / 3$ timing) OR (feed ${ }^{\star} W / 3$ pattern) OR (feed ${ }^{\star} W / 3$ style) OR (feed ${ }^{\star} W / 3$ arrangement $^{\star}$ ) OR (feed ${ }^{\star} W / 3$ environment) OR (feed ${ }^{\star}$ W/3 flavour) OR (feed ${ }^{\star} W / 3$ taste) OR (feed* W/3 content) OR (feed* W/3 composition) OR (feed* W/3 density) OR (feed ${ }^{\star} W / 3$ appear $^{\star}$ ) OR (feed ${ }^{\star} W / 3$ presentation) OR (feed ${ }^{\star} W / 3$ size) OR(feed ${ }^{\star} W / 3$ portion) OR(feed ${ }^{\star} W / 3$ amount) OR(feed ${ }^{\star} W / 3$ fortifi $\left.^{\star}\right)$ OR $_{\left(f^{*}\right.}$ (feed ${ }^{\star}$ W/3 supplement ${ }^{\star}$ ) OR (feed ${ }^{\star} W / 3$ assist $\left.^{\star}\right)$ OR (feed ${ }^{\star} W / 3$ help $\left.^{\star}\right)$ OR (feed ${ }^{\star} W / 3$ support $\left.^{\star}\right)$ OR (feed ${ }^{\star} W / 3$ add $^{\star}$ ) OR (feed* W/3 extra) OR

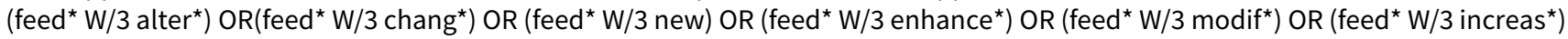
OR (food W/3 decreas $\left.{ }^{\star}\right)$ OR (feed ${ }^{\star}$ W/3 improv* ${ }^{\star}$ OR (feed ${ }^{\star}$ W/3 reduc $\left.^{\star}\right)$ OR (feed f $^{\star} / 3$ target $\left.\left.\left.^{\star}\right)\right)\right)$ )

$\# 2$

OR ((TITLE-ABS-KEY ((nutri ${ }^{\star}$ W/3 content) OR (nutri ${ }^{\star}$ W/3 composition)OR (nutri ${ }^{\star}$ W/3 density))) OR (TITLE-ABS-KEY((diet ${ }^{\star}$ W/3 content) OR (diet* W/3 composition)OR (diet ${ }^{\star}$ W/3 density))) OR (TITLE-ABS-KEY(nutri* W/3 fortifi*)) OR (TITLE-ABS-KEY(diet ${ }^{\star}$ W/3 fortifi*)) OR (TITLE-ABS-KEY(nutri ${ }^{\star}$ W/3 supplement $\left.\left.{ }^{\star}\right)\right)$ OR (TITLE-ABS-KEY (diet* W/3 supplement $\left.{ }^{\star}\right)$ ) OR (TITLE-ABS-KEY (nutri* W/3 add $\left.{ }^{\star}\right)$ OR (nutri* W/3 extra)) OR (TITLE-ABS-KEY((diet* W/3 add*) OR (diet* W/3 extra))))

\#3

OR ((TITLE-ABS-KEY ((dining* W/3 time*) OR (dining* W/3 timing) OR (dining ${ }^{\star} W / 3$ pattern) OR (dining* W/3 style) OR (dining* W/3 arrangement $\left.{ }^{\star}\right)$ OR (dining*W/3 environment))) OR (TITLE-ABS-KEY((dining* W/3 alter ${ }^{\star}$ ) OR (dining* W/3 chang $\left.{ }^{\star}\right)$ OR (dining* W/3 new) OR (dining* W/3 enhance ${ }^{\star}$ ) OR (dining* W/3 modif*) OR (dining*W/3 increas ${ }^{\star}$ ) OR (food W/3 decreas*) OR (dining ${ }^{\star}$ W/3 improv*) OR (dining ${ }^{\star} W / 3$ reduc $\left.^{\star}\right)$ OR (dining $\left.\left.\left.W / 3 \operatorname{target}^{\star}\right)\right)\right)$ )

\#4

OR ((TITLE-ABS-KEY((screening W/3 nutri $\left.{ }^{\star}\right)$ OR (screening W/3 diet*))) OR (TITLE-ABS-KEY((monitoring W/3 nutri $\left.{ }^{\star}\right)$ OR (monitoring W/3 diet $\left.\left.{ }^{\star}\right)\right)$ ) OR (TITLE-ABS-KEY((screening W/3 add $\left.{ }^{\star}\right)$ OR (screening W/3 extra))) OR (TITLE-ABS-KEY ((monitoring W/3 add $\left.{ }^{\star}\right)$ OR (monitoring W/3 extra))) OR (TITLE-ABS-KEY((screeningW/3 alter*) OR (screening W/3 chang*) OR (screening W/3 new) OR (screening W/3 enhance ${ }^{\star}$ ) OR (screeningW/3 modif*) OR (screening W/3 increas ${ }^{\star}$ ) OR (food W/3 decreas ${ }^{\star}$ ) OR (screening W/3 improv ${ }^{\star}$ ) OR (screening W/3 reduc ${ }^{\star}$ ) OR (screening W/3 target $\left.\left.{ }^{\star}\right)\right)$ ) OR (TITLE-ABS-KEY((monitoring W/3 alter $)$ OR (monitoringW/3 chang $\left.{ }^{\star}\right)$ OR (monitoringW/3 new)OR (monitoringW/3 enhance ${ }^{\star}$ )OR (monitoringW/3 modif*)OR (monitoringW/3 increas*)OR (foodW/3 decreas*)OR (monitoringW/3 improv $\left.{ }^{\star}\right) \mathrm{OR}\left(\right.$ monitoringW/3 reduc $\left.{ }^{\star}\right) \mathrm{OR}\left(\right.$ monitoringW/3 $\left.\left.\left.\operatorname{target}{ }^{\star}\right)\right)\right)$ )

\#5

OR ((TITLE-ABS-KEY ((documentation W/3 alter $\left.{ }^{\star}\right)$ OR (documentation W/3 chang*) OR (documentation W/3 new) OR (documentation W/3 enhance ${ }^{\star}$ ) OR (documentation W/3 modif*) OR (documentation W/3 increas ${ }^{\star}$ ) OR (food W/3 decreas ${ }^{\star}$ ) OR (documentation W/3 improv*)OR (documentation W/3 reduc $\left.{ }^{\star}\right)$ OR (documentation W/3 target $\left.\left.{ }^{\star}\right)\right)$ ) OR (TITLE-ABS-KEY ((communication W/3 alter $\left.{ }^{\star}\right)$ OR (communicationW/3 chang*) OR (communication W/3 new) OR (communication W/3 enhance ${ }^{\star}$ ) OR (communication W/3 modif*) OR (communication W/3 increas*) OR (food W/3 decreas*) OR (communication W/3 improv*) OR (communication W/3 reduc ${ }^{\star}$ ) OR (communication $\mathrm{W} / 3$ target $\left.\left.\left.^{\star}\right)\right)\right)$ )

\#6

OR ((TITLE-ABS-KEY ((staff* W/3 nurs*)OR (train* W/3 nurs $\left.\left.{ }^{\star}\right)\right)$ ) OR (TITLE-ABS-KEY((staff* W/3 healthcare)OR (train*W/3 healthcare)OR (staff* W/3 health care) OR (train* W/3 healthcare))) OR (TITLE-ABS-KEY(staff* W/3 cater $\left.\left.{ }^{\star}\right)\right)$ OR (TITLE-ABS-KEY ((staff* W/3 assist $\left.{ }^{\star}\right)$ OR 
(Continued)

(train* W/3 assist) OR (staff* W/3 help*) OR (train* W/3 help*) OR (staff* W/3 support*) OR (train*W/3 support ${ }^{\star}$ ) OR (staff* W/3 add*) OR (train* W/3 add ${ }^{\star}$ ) OR (staff* W/3 extra) OR (train* W/3 extra))) OR (TITLE-ABS-KEY((staff* W/3 alter $\left.{ }^{\star}\right)$ OR (staff* W/3 chang*) OR (staff* W/3 new) OR (staff* W/3 enhance ${ }^{\star}$ )OR (staff* W/3 modif*)OR (staff* W/3 increas ${ }^{\star}$ )OR (food W/3 decreas*)OR (staff* W/3 improv*)OR (staff* W/3 reduc $\left.{ }^{\star}\right)$ OR (staff* W/3 target $\left.\left.{ }^{\star}\right)\right)$ ) OR (TITLE-ABS-KEY ((train* W/3 alter*) OR (train* W/3 chang ${ }^{\star}$ ) OR (train* W/3 new) OR (train* W/3 enhance ${ }^{\star}$ )OR (train ${ }^{\star}$ W/3 modif*)OR (train ${ }^{\star}$ W/3 increas ${ }^{\star}$ )OR (food W/3 decreas ${ }^{\star}$ )OR (train ${ }^{\star}$ W/3 improv*)OR (train W/3 reduc $^{\star}$ )OR

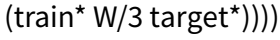

\#7

OR (TITLE-ABS-KEY((supplement* W/3 add $\left.{ }^{\star}\right)$ OR (supplement* W/3 extra)))))

\#8

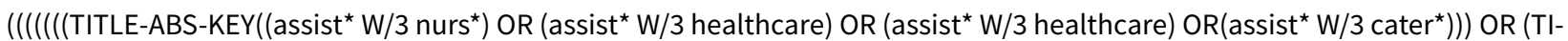

TLE-ABS-KEY ((help* W/3 nurs* $)$ OR (help* W/3 healthcare)OR (help ${ }^{\star}$ W/3 health care) OR (help* W/3 cater $\left.\left.{ }^{\star}\right)\right)$ ) OR (TITLE-ABS-KEY((support $^{\star}$ W/3 nurs ${ }^{\star}$ ) OR (support* W/3 healthcare) OR (support* W/3 health care) OR (support* W/3 cater $\left.\left.\left.{ }^{\star}\right)\right)\right)$ ) \#9

OR (TITLE-ABS-KEY(("protected meal" OR "red tray")) OR ((supportive W/3 intervention) OR (nutrition* W/3intervention) OR (diet* W/3intervention)))

\#10

\#1 OR \#2 OR \#3 OR \#4 OR \#5 OR \#6 OR \#7 OR \#8 OR \#9

\#11

OR (TITLE-ABS-KEY("low BMI" OR "low body mass index"))

\#12

OR (TITLE-ABS-KEY("low weight" OR underweightOR "under-weight"))

\#13

OR (TITLE-ABS-KEY $\left(\right.$ maln $\left.\left.^{\star}\right)\right)$

\#14

OR (TITLE-ABS-KEY(("nutritional risk") OR (risk W/3 maln*)))

$\# 15$

OR (TITLE-ABS-KEY("poor nutr*"OR undernourish* OR "under-nourish *"))

\#16

OR (TITLE-ABS-KEY ((poor W/3 intake*) OR (inadequateW/3 intake ${ }^{\star}$ ) OR (suboptimal W/3 intake*)))

\#17

OR (TITLE-ABS-KEY(institutionali?ed OR elderly))

\#18

OR (TITLE-ABS-KEY(homebound OR "home bound" OR housebound OR "house bound"))

\#19

OR (ABS((extended W/1care) OR(longterm W/1care) OR("long term" W/1 care) OR (community W/1 care)))

\#20

OR (ABS((nursing W/1 home)OR (care W/3 home) OR (residential W/3 home)))

\#21

OR (ABS(inpatient* OR hospitali?* OR "hospital patient*"))

\#22

OR (ABS("nutritional status" OR "nutrition disorder*" OR "nutrition assessment*" OR "nutritional support*" OR "nutrition policy"))

\#23

OR (ABS(diet* OR "food service" OR "energy intake" OR "fortified food"))

$\# 24$

\#11 OR \#12 OR \#13 OR \#14 OR \#15 OR \#16 OR \#17

\#25

\#18 OR \#19 OR \#20 OR \#21 OR \#22 OR \#23

\#26

\#24 OR \#25

\#27

\#10 AND \#26

\#28

((ABS("controlled trial*" OR "controlled clinical trial*" OR "clinical trial*")) OR (ABS(random* ORplacebo)) OR (ABS("meta-analys"

OR metaanalys* OR hta OR "health technology assessment")) OR (ABS(literature* OR "medical database*" OR medline OR pubmed OR embase OR cochrane OR cinahl OR psycinfo OR psyclit OR healthstar OR biosis OR "current content*" OR "systematic review" $\left.{ }^{\star}\right)$ ) \#29

\#27 AND \#28

\#30

$\left(\right.$ ABS $\left(\right.$ adult $\left.\left.^{\star}\right)\right)$

\#31

\#29 AND \#30

Supportive interventions for enhancing dietary intake in malnourished or nutritionally at-risk adults (Review) 
(Continued)

\#32

(ABS(pregnan $\left.\left.{ }^{\star}\right)\right)$

\#33

\#31 AND NOT \#32

\#34

$\left(\right.$ ABS(animal $\left.\left.{ }^{\star}\right)\right)$

\#35

\#33 AND NOT \#34

\section{ISI Web of Science}

\#1 TS=((food ${ }^{\star}$ OR meal ${ }^{\star}$ OR snack* OR drink ${ }^{\star}$ OR feed $\left.{ }^{\star}\right)$ NEAR/3 (time* OR timing OR pattern OR style OR arrangement* OR environment OR flavor OR taste OR content OR composition OR density OR appear ${ }^{\star}$ OR presentation OR size OR portion OR amount OR fortifi ${ }^{\star}$ OR supplement ${ }^{\star}$ OR assist* OR help* OR support ${ }^{\star}$ OR add ${ }^{\star}$ OR extra OR alter ${ }^{\star}$ OR chang ${ }^{\star}$ OR new OR enhance* OR modif ${ }^{\star}$ OR increas $^{\star}$ OR decreas* OR improv* OR reduc ${ }^{\star}$ OR target $\left.\left.{ }^{\star}\right)\right)$

\# 2 TS=((nutri* OR diet*) NEAR/3 (content OR composition OR density OR fortfi ${ }^{\star}$ OR supplement* OR add ${ }^{\star}$ OR extra OR alter ${ }^{\star}$ OR chang* OR new OR enhance* OR modif* OR increas* OR decreas* OR improv* OR reduc* OR target))

\# 3 TS=((dining $\left.{ }^{\star}\right)$ NEAR/3 (time* OR timing OR pattern OR style OR arrangement* OR environment OR alter* OR chang* OR new OR enhance ${ }^{\star}$ OR modif* OR increas ${ }^{\star}$ OR decreas ${ }^{\star}$ OR improv* OR reduc $^{\star}$ OR target $\left.{ }^{\star}\right)$ )

\# 4 TS=((screening OR monitoring) NEAR/3 (nutri^ OR diet* OR add ${ }^{\star}$ OR extra OR alter ${ }^{\star}$ OR chang ${ }^{\star}$ OR new OR enhance* OR modif OR increas ${ }^{\star}$ OR decreas ${ }^{\star}$ OR improv* OR reduc ${ }^{\star} \mathrm{OR}^{*}$ target $\left.\left.{ }^{\star}\right)\right)$

\# 5 TS=((documentation OR communication) NEAR/3 (alter ${ }^{\star}$ OR chang ${ }^{\star}$ OR new OR enhance* OR modif ${ }^{\star}$ OR increas ${ }^{\star}$ OR decreas ${ }^{\star}$ OR improv $^{\star}$ OR reduc OR target $\left.\left.^{\star}\right)\right)$

\# 6 TS=((staff* OR train $\left.{ }^{\star}\right)$ NEAR/3 (nurs ${ }^{\star}$ OR healthcare OR "health care" OR cater ${ }^{\star}$ OR assist* OR help* OR support* OR add OR extra OR alter ${ }^{\star}$ OR chang ${ }^{\star}$ OR new OR enhance OR modif $^{\star}$ OR increas ${ }^{\star}$ OR decreas ${ }^{\star}$ OR improv OR reduc $^{\star}$ OR target $\left.\left.^{\star}\right)\right)$

\# 7 TS $=\left(\left(\right.\right.$ supplement $\left.{ }^{\star}\right)$ NEAR/6 (add ${ }^{\star}$ OR extra))

\# 8 TS=((assist* OR help* OR support $\left.{ }^{\star}\right)$ NEAR/3 (nurs* OR healthcare OR "health care" OR cater $\left.\left.{ }^{\star}\right)\right)$

\# 9 TS=(("protected meal" OR "red tray") OR ((supportive OR nutrition* OR diet* ${ }^{\star}$ NEAR/3 intervention*))

\# 10 \#9 OR \#8 OR \#7 OR \#6 OR \#5 OR \#4 OR \#3 OR \#2 OR \#1

\# $11 \mathrm{TS}=(($ "low bmi" OR "low body mass index"))

\# 12 TS=(("low weight" OR underweight OR "under-weight"))

\# $13 \mathrm{TS}=\left(\mathrm{maln}^{\star}\right)$

\# 14 TS=("nutritional risk" OR (risk NEAR/3 maln*))

\# 15 TS=(("poor nutr*" OR undernourish* OR "under nourish*"))

\# 16 TS=((poor OR inadequate OR suboptimal) NEAR/6 intake*)

\# 17 TS=((institutionali?ed OR elderly $))$

\# 18 TS=((homebound OR "home bound" OR housebound OR "house bound"))

\# 19 TS=((extended OR longterm OR "long term" OR community) NEAR/1 care)

\# $20 \mathrm{TS}=(($ nursing OR care OR residential) NEAR/1 home)

\# 21 TS=((inpatient ${ }^{\star}$ OR hospitali?* OR "hospital patient $\left.\left.{ }^{\star} "\right)\right)$

\# $22 \mathrm{TS}=$ (nutritional status)

\# 23 TS=(nutrition disorder*)

\# $24 \mathrm{TS}=\left(\left(\right.\right.$ "nutrition assessment ${ }^{\star}$ " OR "nutritional support*"OR "nutrition policy"))

\# 25 TS=((diet* OR "food service" OR "energy intake" OR "fortified food"))

\# 26 \#25 OR \#24 OR \#23 OR \#22 OR \#21 OR \#20 OR \#19 OR \#18 OR \#17 OR \#16 OR \#15 OR \#14 OR \#13 OR \#12 OR \#11

\# 27 \#26 AND \#10

\# 28 TS=("controlled trial*" OR "controlled clinical trial*" OR "clinical trial*")

\# 29 TS=(random* OR placebo)

\# 30 TS=("meta-analys*" OR metaanalys* OR hta OR "health technology assessment")

\# 31 TS=((literature ${ }^{\star}$ OR "medical database*" OR medline OR pubmed OR embase OR cochrane OR cinahl OR psycinfo OR psyclit OR healthstar OR biosis OR "current content*" OR "systematic review" $\left.{ }^{\star}\right)$ )

\# 32 \#31 OR \#30 OR \#29 OR \#28

\# 33 \#32 AND \#27

\# $34 \mathrm{TS}=\left(\right.$ adult $\left.{ }^{\star}\right)$

\# 35 \#34 AND \#33

\# 36 TS=(pregnan $\left.{ }^{\star}\right)$

\# 37 \#35 NOT \#36

\# 38 TS $=\left(\right.$ animal $\left.{ }^{*}\right)$

\# 39 \#37 NOT \#38 


\section{Appendix 2. Search strategies (January 2013 to September 2016)}

\section{Cochrane Library (Wiley)}

\section{l: Population}

1. [mh^Stroke] or stroke:ti,ab

2. [mh^"Alzheimer Disease"] or alzheimer:ti,ab

3. $\left[\mathrm{mh}^{\wedge}{ }^{\wedge}\right.$ Dementia] or dement ${ }^{\star}: t i, a b$

4. [mh ^"Mild Cognitive Impairment"] or "cognitive impairment":ti,ab

5. [mh "Hip Fractures"] or ("hip fracture*" or "femoral neck fracture*"):ti,ab

6. [mh^^"Nursing Homes"] or [mh^^"Homes for the Aged"] or ("nursing home*"):ti,ab

7. (residents or residential):ti,ab

8. [mh^"Aged"] or [mh^^"Aging"] or aged:ti,ab

9. [mh^"Frail Elderly"] or (elder or elders or elderly):ti,ab

10. (older or geriatric):ti,ab

11. [mh^^"Inpatients"] or inpatients:ti,ab

12. [mh^"Outpatients"] or outpatients:ti,ab

13. [mh^^"Institutionalization"] or institutionali^:ti,ab

14. [mh^"Hospitalization"] or (hospitali?ed or hospitali?ation):ti,ab

15. $\{$ or \#1-\#14\}

\section{II: Condition}

16. [mh^"Malnutrition"] or [mh ^"Protein-Energy Malnutrition"]

17. (malnourish ${ }^{\star}$ or malnutrition):ti,ab

18. [mh^"Nutrition Assessment"]

19. [mh ^"Nutritional Status"] or "nutritional status":ti,ab

20. [mh^"Nutritional Requirements"]

21. [mh^"Nutrition Disorders"]

22. [mh^"Nutritional Support"]

23. ((nutritional or nutrition or nutritionally) near/2 risk):ti,ab

24. ((unintentional or risk) near/2 "weight loss"):ti,ab

25. (undernutrition or undernourished or hyponutrition):ti,ab

26. [mh^^"Elder Nutritional Physiological Phenomena"]

27. [mh^^"Energy Intake"]

28. [mh^^"Feeding Behavior"] or [mh^^"Feeding Methods"] 
(Continued)

29. ("Mini Nutritional Assessment" or "Eating Behaviour Scale" or "Edinburgh Feeding Evaluation" or "Malnutrition Universal Screening Tool"):ti,ab

30. ((improve* or increase* or inadequate) near/3 ("nutrient intake" or "energy intake" or "dietary intake" or "food intake")):ti,ab

31. $\{$ or \#16-\#30\}

32. \#15 and \#31

33. \#32 not (child* or infant $^{\star}$ or pregnan $\left.{ }^{\star}\right): t i, a b, k w$

34. Publication Year from 2013 to 2016

\section{MEDLINE (Ovid SP)}

\section{I:Population}

1. Stroke/ or stroke.tw.

2. Alzheimer Disease/ or alzheimer.tw.

3. Dementia/ or dement*.tw.

4. Mild Cognitive Impairment/ or cognitive impairment.tw.

5. exp Hip Fractures/ or (hip fracture? or femoral neck fracture?).tw.

6. Nursing Homes/ or Homes for the Aged/ or (nursing home?).tw.

7. (residents or residential).tw.

8. Aged/ or Aging/ or aged.tw.

9. Frail Elderly/ or (elder or elders or elderly).tw.

10. (older or geriatric).tw.

11. Inpatients/ or inpatients.tw.

12. Outpatients/ or outpatients.tw.

13. Institutionalization/ or institutionali ${ }^{\star}$. tw.

14. Hospitalization/ or (hospitali?ed or hospitali?ation).tw.

15. or/1-14

II: Condition

16. Malnutrition/ or Protein-Energy Malnutrition/

17. (malnourish* or malnutrition).tw.

18. Nutrition Assessment/

19. Nutritional Status/ or nutritional status.tw.

20. Nutritional Requirements/

21. Nutrition Disorders/

22. Nutritional Support/

23. ((nutritional or nutrition or nutritionally) adj2 risk).tw.

24. ((unintentional or risk) adj2 weight loss).tw. 
(Continued)

25. (undernutrition or undernourished or hyponutrition).tw.

26. Elder Nutritional Physiological Phenomena/

27. Energy Intake/

28. Feeding Behavior/ or Feeding Methods/

29. (Mini Nutritional Assessment or Eating Behaviour Scale or Edinburgh Feeding Evaluation or Malnutrition Universal Screening Tool).tw.

30. ((improve* or increase? or inadequate) adj3 (nutrient intake or energy intake or dietary intake or food intake)).tw.

31. or $/ 16-30$

32. 15 and 31

III. [Cochrane Handbook 2008 RCT filter - sensitivity and precision max. version]

33. randomized controlled trial.pt.

34. controlled clinical trial.pt.

35. randomi?ed.ab.

36. placebo.ab.

37. clinical trials as topic/

38. randomly.ab.

39. trial.ti.

40. or/33-39

41. exp animals/ not humans/

42. 40 not 41

43. 32 and 42

44. 43 not (child* or infant* or pregnan*).tw.

45. limit 44 to $y r=" 2013-C u r r e n t "$

ClinicalTrials.gov (Advanced search)

Search Terms: malnourished OR malnutrition OR undernourished OR undernutrition OR "under nutrition" OR "poor nutritional status" OR "nutritional risk" OR "inadequate nutrient intake"

Study Type: Interventional Studies

Age Group: Adult (18-65), Senior (66+)

\section{WHO ICTRP (Standard search)}

malnourished AND elder* OR

malnutrition AND elder ${ }^{\star}$ OR

undernourished AND elder ${ }^{\star}$ OR

undernutrition AND elder* OR

malnourished AND aged OR 
(Continued)

malnutrition AND aged OR

undernourished AND aged OR

undernutrition AND aged OR

malnourished AND geriatric OR

malnutrition AND geriatric OR

undernourished AND geriatric OR

undernutrition AND geriatric

\section{Appendix 3. Description of interventions}

\section{Intervention(s)}

Barton 2000

I1: portion size decreased by $20 \%$ but fortified to achieve overall daily energy provision increased by $200 \mathrm{kcal}$ (randomised)

\section{Type of intervention(s) ${ }^{a} \quad$ Comparator(s)}

Modification of meal pro- Normal hospital file or pattern menu (randomised group)

12: normal hospital menu plus cooked breakfast (not randomised group)

Beck 2002

I1: homemade oral supplement (group A, not randomised)

Additional supplementa- Usual diet tion of meals

12: homemade oral supplement (group B)

Bouillanne $\mathbf{2 0 1 3} \quad$ 'Pulse diet': 78\% of daily protein requirements provided at lunch (no change to energy and protein)
Modification of meal profile or pattern
'Spread diet': usual diet (daily protein requirements distributed between meals)

Additional supplementa- Standard diet tion of meals

Changes to the feeding Activities only environment

Modification of meal pro- $\quad$ Routine care, no file or pattern meals enhanced

\begin{tabular}{llll}
\hline Castellanos 2009 & $\begin{array}{l}\text { I1: two breakfast and two lunch foods fortified to improve } \\
\text { energy and protein content (hot cereal and juice break- } \\
\text { fast, soup and side dish at lunch) }\end{array}$ & $\begin{array}{l}\text { Modification of meal pro- } \\
\text { file or pattern }\end{array}$ & $\begin{array}{l}\text { Routine care, no } \\
\text { meals enhanced }\end{array}$ \\
\cline { 2 - 4 } 2: two lunch foods only fortified versus normal menu & $\begin{array}{l}\text { Changes to the organisa- } \\
\text { tion of nutritional care }\end{array}$ & No training \\
\hline Chang 2005 & $\begin{array}{l}\text { Training in feeding skills (feeding skills training pro- } \\
\text { gramme for nursing assistants) }\end{array}$ & $\begin{array}{l}\text { Additional supplementa- } \\
\text { tion of meals }\end{array}$ & $\begin{array}{l}\text { Normal hospital di- } \\
\text { et }\end{array}$ \\
\hline Dennis 2005 & $\begin{array}{l}\text { Additional personal attention of a dietetic assistant e.g. } \\
\text { checking personal food preferences, assisting with food } \\
\text { choice, provision of appropriate feeding aids, feeding as- }\end{array}$ & $\begin{array}{l}\text { Changes to the organisa- } \\
\text { tion of nutritional care }\end{array}$ & Routine care \\
\hline
\end{tabular}


sistance and collecting information to aid nutritional as-

sessment

\begin{tabular}{|c|c|}
\hline \multirow[t]{4}{*}{ Essed 2007} & $\begin{array}{l}\text { Food sprinkled with } 1 \mathrm{~g}(+0.2 \mathrm{~g}) \text { of intervention + mal- } \\
\text { todextrin carrier }\end{array}$ \\
\hline & I1: monosodium glutamate \\
\hline & 12: flavour \\
\hline & 13: monosodium glutamate + flavour \\
\hline
\end{tabular}

\section{Essed 2009}

Three foods (previously identified as preferred), i.e.

Modification of meal pro- Maltodextrin file or pattern mashed potato $(0.2 \mathrm{~g} \mathrm{NaCl} / 100 \mathrm{~g}+0.5 \%$ monosodium glutamate), mince meat $(0.37 \mathrm{~g} \mathrm{NaCl} / 100 \mathrm{~g}+2 \%$ monosodium glutamate and spinach $(0.25 \mathrm{~g} \mathrm{NaCl} / 100 \mathrm{~g}+2 \%$ monosodium glutamate)

mashed potato $(0.2 \mathrm{~g} \mathrm{NaCl} / 100 \mathrm{~g}+0.5 \%$ monosodium glu-
tamate), mince meat $(0.37 \mathrm{~g} \mathrm{NaCl} / 100 \mathrm{~g}+2 \%$ monosodi-
um glutamate and spinach $(0.25 \mathrm{~g} \mathrm{NaCl} / 100 \mathrm{~g}+2 \%$
monosodium glutamate)

Faxen-Irving 2011 A daily dose of $3 \times 30 \mathrm{~mL}$ fat emulsion distributed at the same time as pharmaceutical prescriptions

\begin{tabular}{|c|c|c|c|}
\hline Faxen-Irving 2011 & $\begin{array}{l}\text { A daily dose of } 3 \times 30 \mathrm{~mL} \text { fat emulsion distributed at the } \\
\text { same time as pharmaceutical prescriptions }\end{array}$ & $\begin{array}{l}\text { Additional supplementa- } \\
\text { tion of meals }\end{array}$ & Standard care \\
\hline Gaskill 2009 & Nutrition education programme & $\begin{array}{l}\text { Changes to the organisa- } \\
\text { tion of nutritional care }\end{array}$ & Usual care \\
\hline Germain 2006 & $\begin{array}{l}\text { Re-formed foods, thickened beverages and dietary sup- } \\
\text { plements as necessary }\end{array}$ & $\begin{array}{l}\text { Modification of meal pro- } \\
\text { file or pattern }\end{array}$ & $\begin{array}{l}\text { Traditional modi- } \\
\text { fied texture diet }\end{array}$ \\
\hline Hankey 1993 & Supplements in addition to their normal hospital diet & $\begin{array}{l}\text { Additional supplementa- } \\
\text { tion of meals }\end{array}$ & $\begin{array}{l}\text { Standard hospital } \\
\text { food }\end{array}$ \\
\hline Hickson 2004 & $\begin{array}{l}\text { Additional nutritional care from a trained health care as- } \\
\text { sistant }\end{array}$ & $\begin{array}{l}\text { Changes to the organisa- } \\
\text { tion of nutritional care }\end{array}$ & Usual care \\
\hline Holyday 2012 & $\begin{array}{l}\text { Malnutrition care plan; screening, assessment and inter- } \\
\text { vention tailored to individuals requirements (including } \\
\text { texture modification, fortification, oral nutritional supple- } \\
\text { ments, snacks, assistance) }\end{array}$ & $\begin{array}{l}\text { Changes to the organisa- } \\
\text { tion of nutritional care }\end{array}$ & Usual care \\
\hline Johansen 2004 & Nutrition team (dietitian + nurse) & $\begin{array}{l}\text { Changes to the organisa- } \\
\text { tion of nutritional care }\end{array}$ & Usual care \\
\hline Kraft 2012 & $\begin{array}{l}\text { Oral nutritional supplements + monitoring using telemed- } \\
\text { icine }\end{array}$ & $\begin{array}{l}\text { Changes to the organisa- } \\
\text { tion of nutritional care }\end{array}$ & Usual care \\
\hline Kretser 2003 & $\begin{array}{l}\text { Modified meals on wheels system ( } 21 \text { meals }+14 \text { snacks) } \\
\text { and daily phone call }\end{array}$ & $\begin{array}{l}\text { Congregate and home } \\
\text { meal delivery systems }\end{array}$ & $\begin{array}{l}\text { Traditional meals } \\
\text { on wheels (one } \\
\text { hot meal delivered } \\
\text { five days a week at } \\
\text { lunch) }\end{array}$ \\
\hline Larsson 1990 & Oral nutritional supplements plus normal hospital diet & $\begin{array}{l}\text { Additional supplementa- } \\
\text { tion of meals }\end{array}$ & $\begin{array}{l}\text { Normal hospital di- } \\
\text { et }\end{array}$ \\
\hline Leslie 2012 & Energy enriched usual meals & $\begin{array}{l}\text { Modification of meal pro- } \\
\text { file or pattern }\end{array}$ & Usual care \\
\hline Lin 2010 & $\begin{array}{l}\text { I1: spaced-retrieval - a method to enhance learning, reten- } \\
\text { tion and recall of information }\end{array}$ & $\begin{array}{l}\text { Changes to the organisa- } \\
\text { tion of nutritional care }\end{array}$ & Usual care \\
\hline
\end{tabular}

\section{Additional supplementa- Standard care} tion of meals

\section{Changes to the organisa- Usual care} Modification of meal pro- Traditional modiAdditional supplementa- Standard hospital Changes to the organisa- Usual care tion of nutritional care vention tailored to individuals requirements (including texture modification, fortification, oral nutritional supplements, snacks, assistance)

\section{Modification of meal pro- Usual hot meal} file or pattern 
(Continued)

12: Montessori intervention - a method capable of stop-

ping or reducing residents problem behaviours

\begin{tabular}{|c|c|c|c|}
\hline Lin 2011 & $\begin{array}{l}\text { Montessori intervention - designed to manage eating diffi- } \\
\text { culties }\end{array}$ & $\begin{array}{l}\text { Changes to the organisa- } \\
\text { tion of nutritional care }\end{array}$ & Usual care \\
\hline Mathey 2001a & $\begin{array}{l}\text { Improved meal ambiance comprising improvements to } \\
\text { physical environment, meal service and organisation of } \\
\text { assistance }\end{array}$ & $\begin{array}{l}\text { Changes to the feeding } \\
\text { environment }\end{array}$ & Usual care \\
\hline Mathey 2001b & Creating a better ambience during food consumption & $\begin{array}{l}\text { Changes to the feeding } \\
\text { environment }\end{array}$ & Usual care \\
\hline Munk 2014 & $\begin{array}{l}\text { Energy and protein enriched foods provided in addition to } \\
\text { the hospital food via an a la carte menu }\end{array}$ & $\begin{array}{l}\text { Modification of meal pro- } \\
\text { file or pattern }\end{array}$ & Usual care \\
\hline Nijs 2006 & $\begin{array}{l}\text { Family-style meals comprising table dressing, food ser- } \\
\text { vice, staff protocols, residents protocol and a meal-time } \\
\text { protocol, meal choice at the time of meal }\end{array}$ & $\begin{array}{l}\text { Changes to the feeding } \\
\text { environment }\end{array}$ & $\begin{array}{l}\text { Individual pre- } \\
\text { plated meal ser- } \\
\text { vice, meal chosen } 2 \\
\text { weeks in advance. }\end{array}$ \\
\hline Olofsson 2007 & Multi-component intervention (including nutrition) & $\begin{array}{l}\text { Changes to the organisa- } \\
\text { tion of nutritional care }\end{array}$ & Usual care \\
\hline \multirow[t]{2}{*}{ Pivi 2011} & 11: nutrition education for caregivers and participants & \multirow{2}{*}{$\begin{array}{l}\text { Changes to the organisa- } \\
\text { tion of nutritional care }\end{array}$} & \multirow[t]{2}{*}{ Usual care } \\
\hline & $\begin{array}{l}\text { 12: oral nutritional supplements (two cartons daily for six } \\
\text { months) }\end{array}$ & & \\
\hline Potter 2001 & Oral nutritional supplement + normal hospital diet & $\begin{array}{l}\text { Additional supplementa- } \\
\text { tion of meals }\end{array}$ & $\begin{array}{l}\text { Normal hospital di- } \\
\text { et }\end{array}$ \\
\hline Remsburg 2001 & Buffet style dining programme for supper only & $\begin{array}{l}\text { Changes to the feeding } \\
\text { environment }\end{array}$ & $\begin{array}{l}\text { Usual care, tray- } \\
\text { style meal served } \\
\text { by nursing home } \\
\text { staff }\end{array}$ \\
\hline Salva 2011 & $\begin{array}{l}\text { Teaching and training intervention to improve nutrition } \\
\text { care }\end{array}$ & $\begin{array}{l}\text { Changes to the organisa- } \\
\text { tion of nutritional care }\end{array}$ & Usual care \\
\hline Silver 2008 & Home-delivered fortified lunch once weekly for 7 months & $\begin{array}{l}\text { Modification of meal pro- } \\
\text { file or pattern }\end{array}$ & $\begin{array}{l}\text { Home deliv- } \\
\text { ered usual lunch } \\
\text { once weekly for } 7 \\
\text { months }\end{array}$ \\
\hline Simmons 2008 & Mealtime feeding assistance and/or between meal snacks & $\begin{array}{l}\text { Additional supplementa- } \\
\text { tion of meals }\end{array}$ & Usual care \\
\hline Simmons 2010 & I1: snacks between meal snacks & Additional supplementa- & Usual care \\
\hline \multirow[t]{2}{*}{ Smolliner 2008} & 12: oral nutritional supplements & & \\
\hline & $\begin{array}{l}\text { Protein and energy-enriched soups and sauces and two } \\
\text { additional snacks high in protein and energy }\end{array}$ & $\begin{array}{l}\text { Modification of meal pro- } \\
\text { file or pattern }\end{array}$ & Usual diet \\
\hline Splett 2003 & $\begin{array}{l}\text { Medical nutrition therapy (protocol-driven nutritional as- } \\
\text { sessment and intervention activities carried by dietitians) }\end{array}$ & $\begin{array}{l}\text { Changes to the organisa- } \\
\text { tion of nutritional care }\end{array}$ & $\begin{array}{l}\text { Usual care by dieti- } \\
\text { tians }\end{array}$ \\
\hline
\end{tabular}


(Continued)

Taylor 2006

5-meal menu pattern with energy content similar to existing 3-meal menu
Modification of meal profile or pattern

Additional supplementation of meals 3-meal menu (usual care)

Van den Berg $2015 \quad$ I1: offered 125 mL ONS twice daily with medication rounds

Usual care $(125 \mathrm{~mL}$ ONS offered in between meals)

12: offered $62 \mathrm{~mL}$ ONS four times daily with medication rounds

\section{Van Ort 1995}

12: offered $62 \mathrm{~mL}$ ONS daily with medication rounds

\section{Changes to the feeding} environment

Usual care

aNumbers refer to intervention sub-categories: (1) changes to the organisation of nutritional care, (2) changes to the feeding environment, (3) modification of meal profile or pattern, (4) additional supplementation of meals, (5) congregate and home meal delivery systems - see Table 1

C: comparator; I: intervention; ONS: oral nutritional supplement 


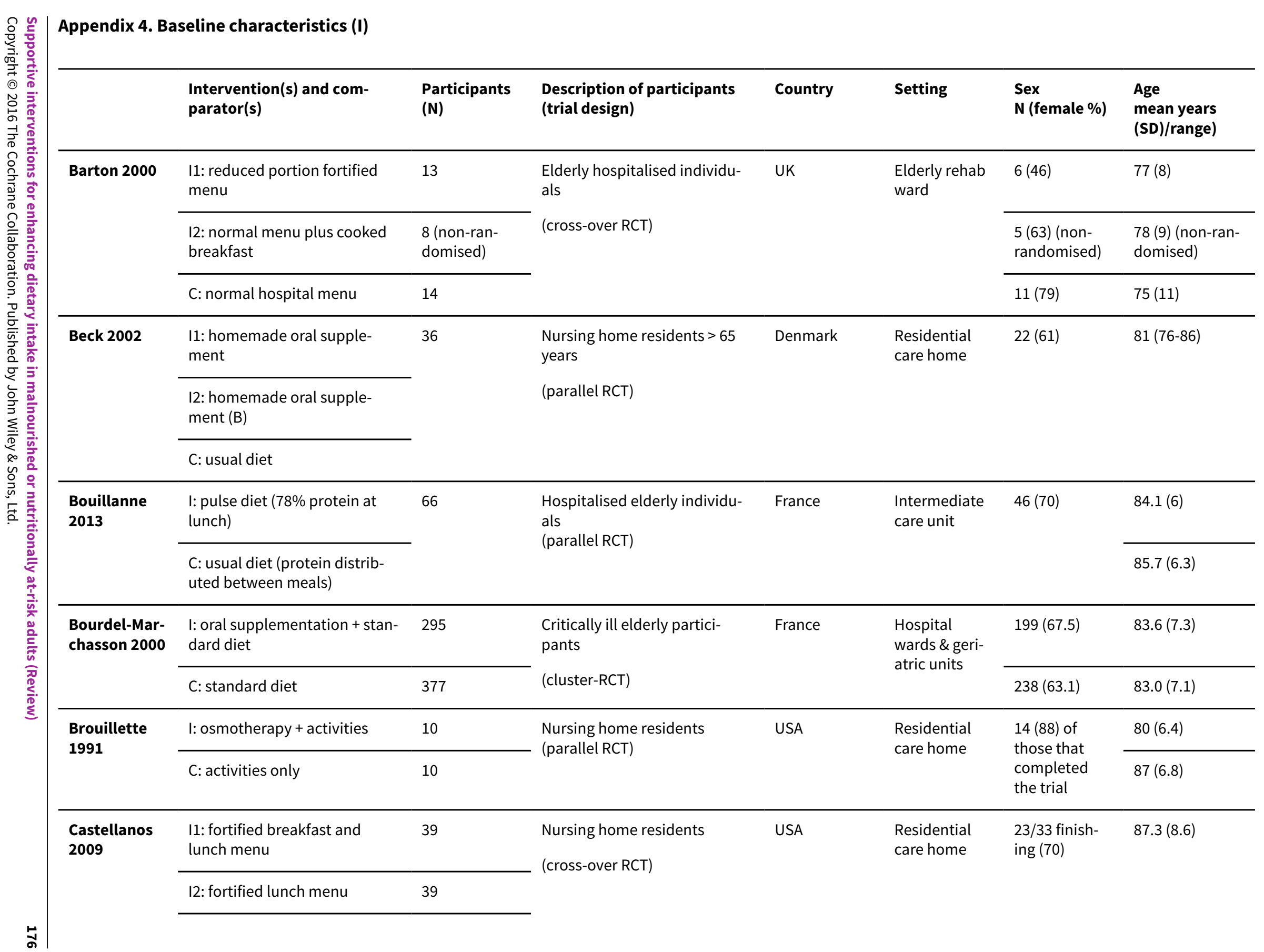




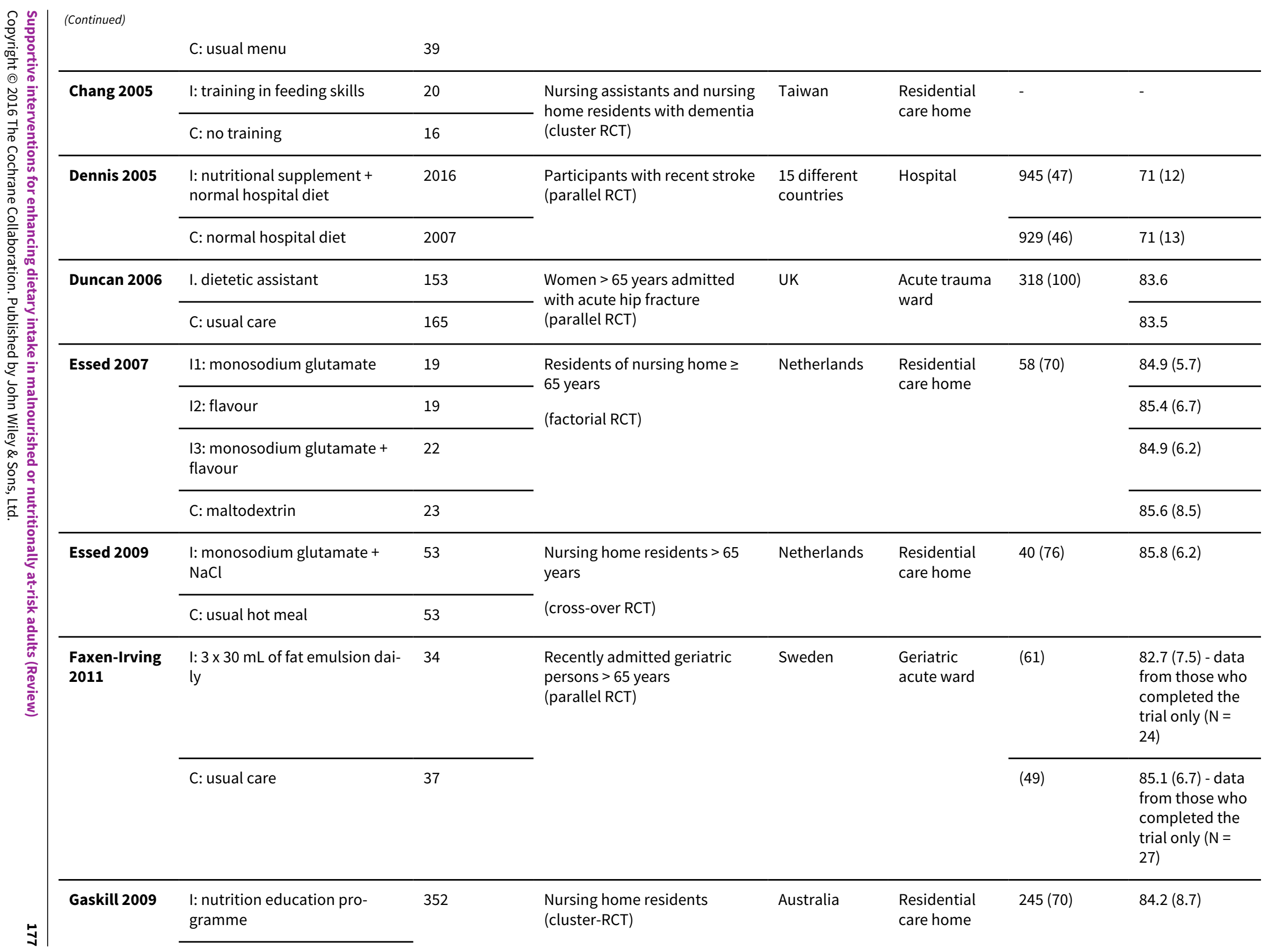




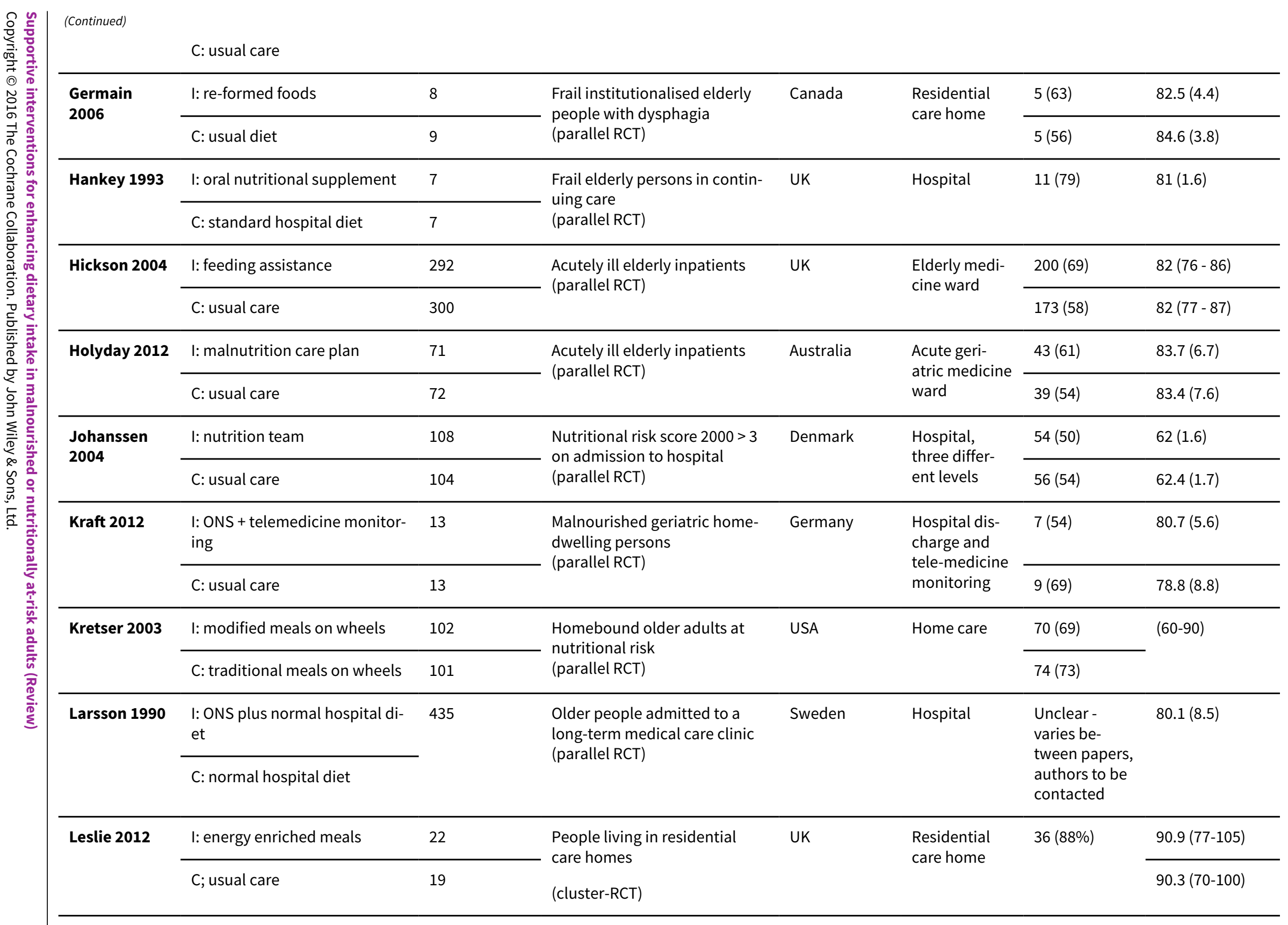




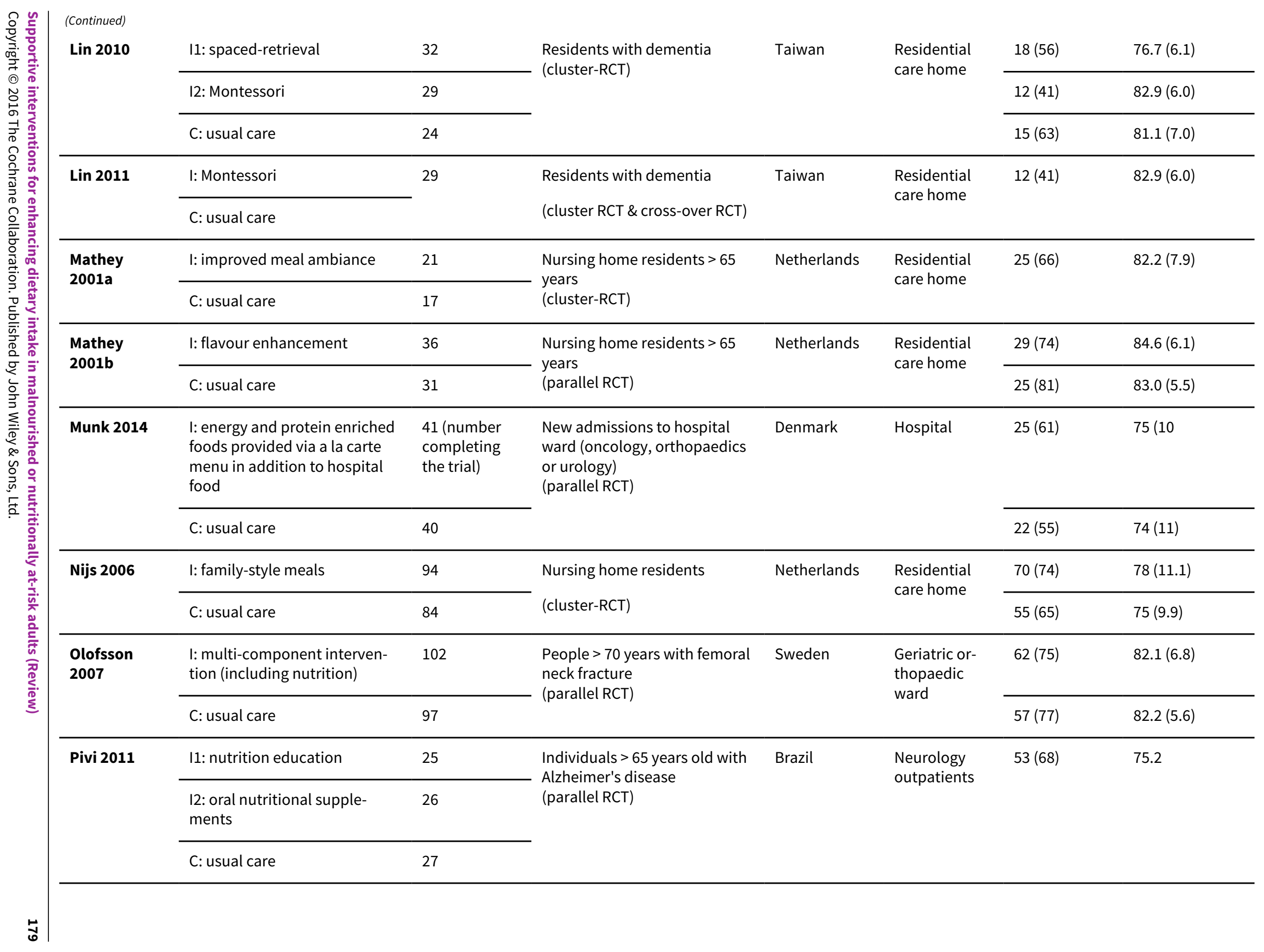




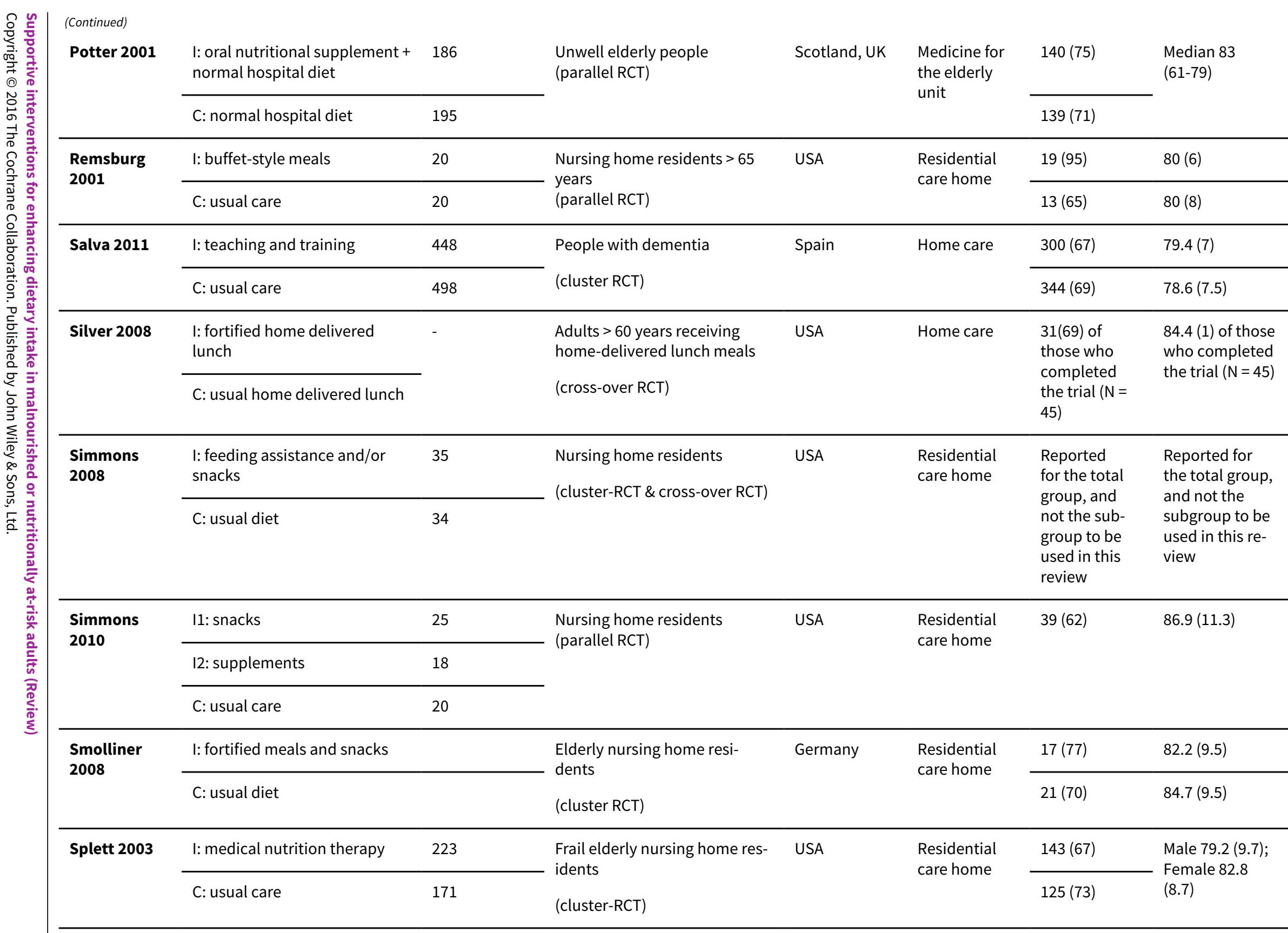




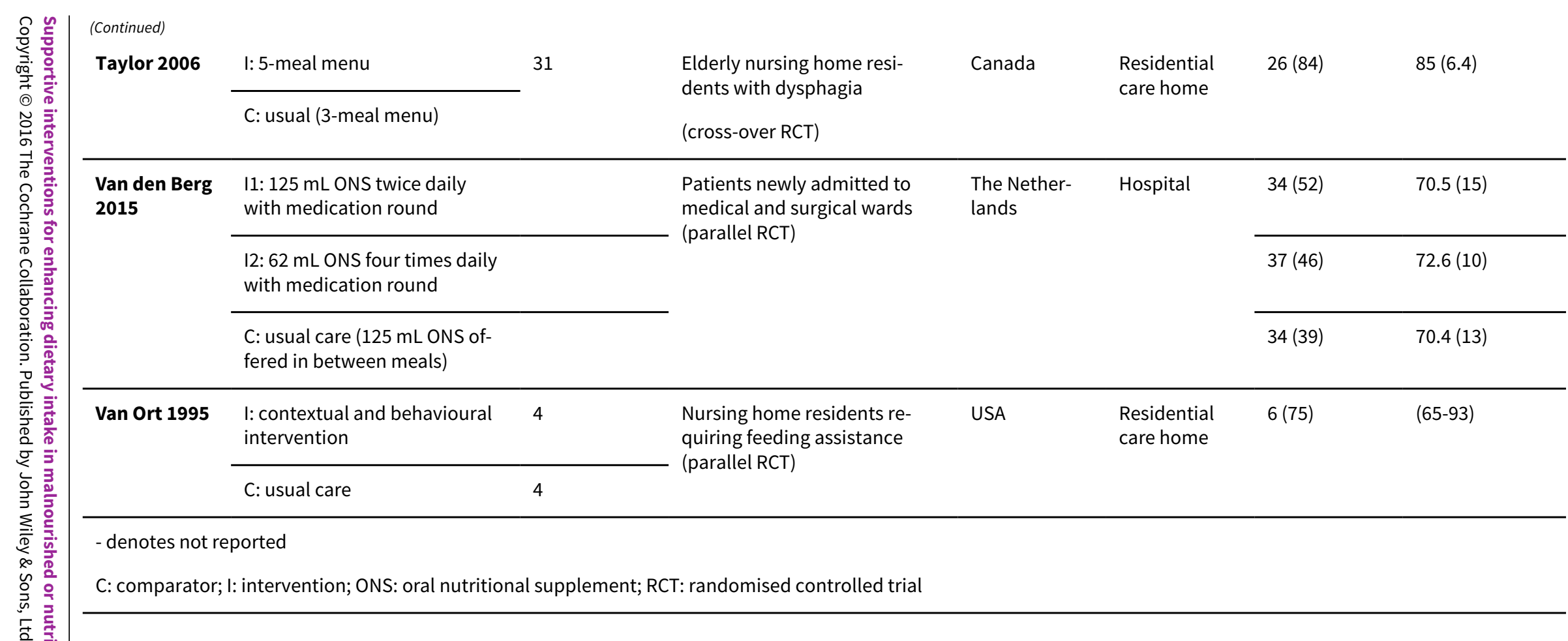




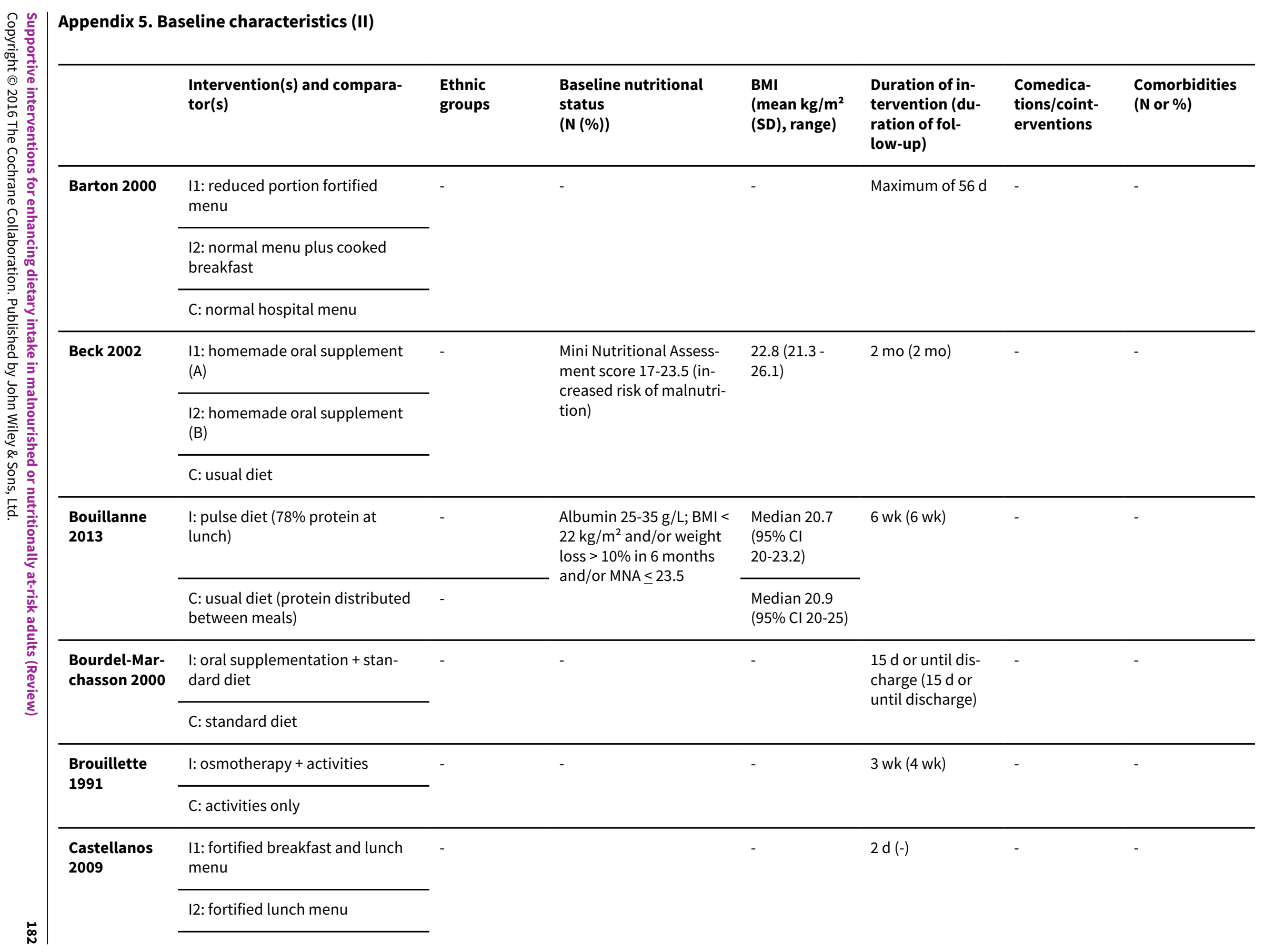




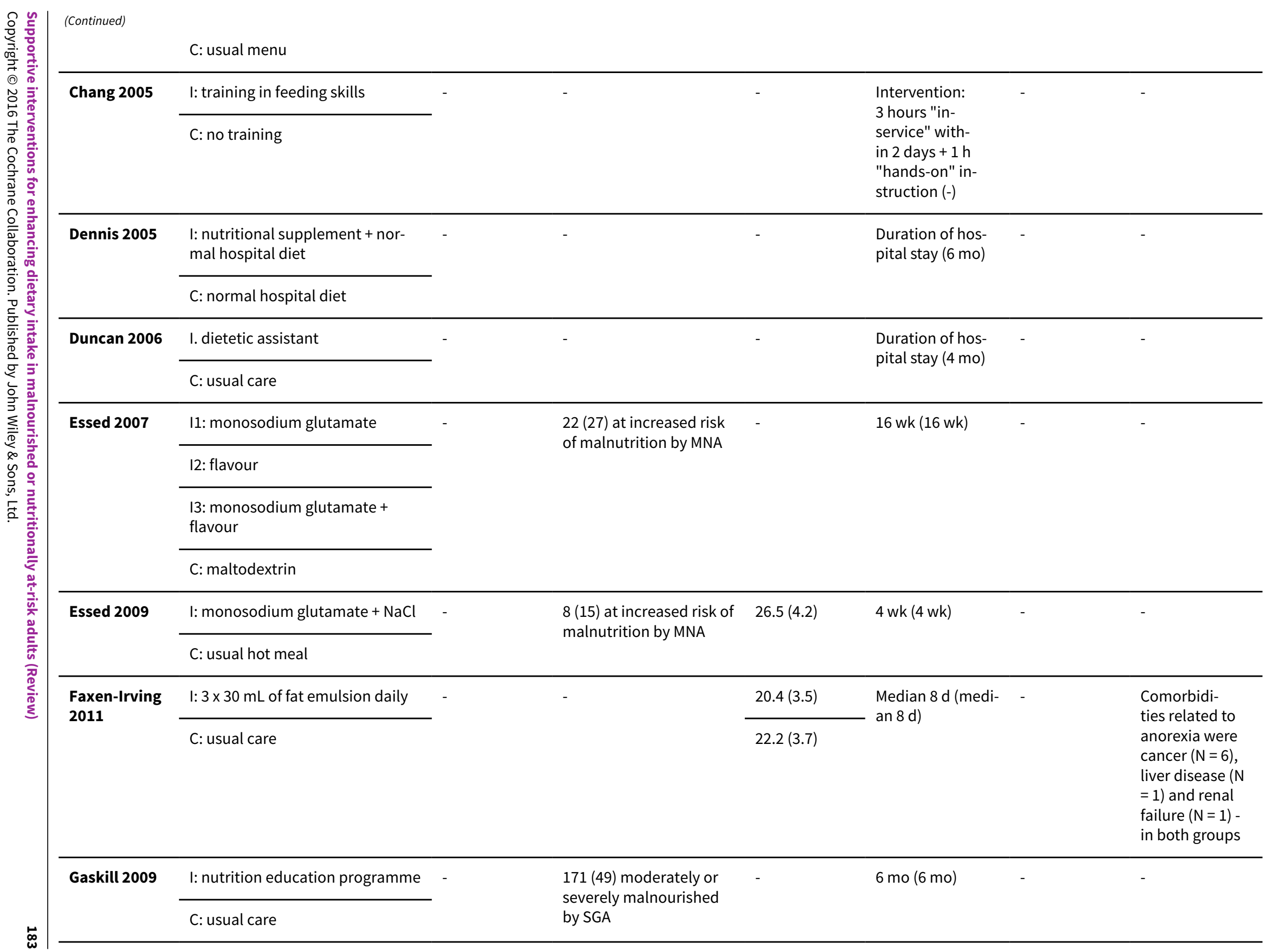




\begin{tabular}{|c|c|c|c|c|c|c|c|c|}
\hline \multirow{2}{*}{\multicolumn{2}{|c|}{ 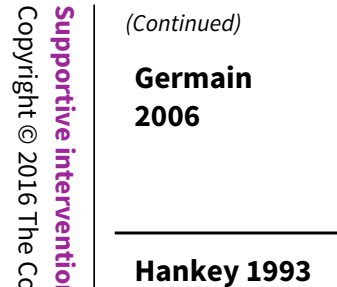 }} & $\begin{array}{l}\text { I: re-formed foods } \\
\text { C: usual diet }\end{array}$ & - & $\begin{array}{l}17(100) \text { unintention- } \\
\text { al weight loss }>7.5 \% \text { in } \\
\text { previous } 3 \text { mo or } \mathrm{BMI}< \\
24 \mathrm{~kg} / \mathrm{m}^{2}\end{array}$ & $\begin{array}{l}22.4(3.9) \\
21.2(2.3)\end{array}$ & $12 \mathrm{wk}(12 \mathrm{wk})$ & - & - \\
\hline & & I: ONS & - & - & - & $8 w k(8 w k)$ & - & - \\
\hline 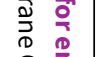 & & C: standard hospital diet & & & & & & \\
\hline 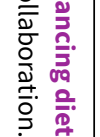 & \multirow[t]{2}{*}{ Hickson 2004} & I: feeding assistance & $\begin{array}{l}282(96.6) \\
\text { white ethnic } \\
\text { group }\end{array}$ & & $\begin{array}{l}21.7 \\
(18.6-25.3)\end{array}$ & \multirow[t]{2}{*}{$\begin{array}{l}\text { Duration of hos- } \\
\text { pital stay (dura- } \\
\text { tion of hospital } \\
\text { stay) }\end{array}$} & \multirow[t]{2}{*}{-} & \multirow[t]{2}{*}{-} \\
\hline 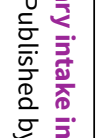 & & C: usual care & $\begin{array}{l}286(95.3) \\
\text { white ethnic } \\
\text { group }\end{array}$ & & $\begin{array}{l}21.8 \\
(19.1-25.7)\end{array}$ & & & \\
\hline 产 & \multirow[t]{2}{*}{ Holyday 2012} & I: malnutrition care plan & \multirow[t]{2}{*}{-} & \multirow{2}{*}{$\begin{array}{l}119(83) \text { malnourished } \\
\text { or at risk of malnutrition } \\
\text { by MNA score }\end{array}$} & $23.8(5.9)$ & \multirow{2}{*}{$\begin{array}{l}\text { Duration of hos- } \\
\text { pital stay (dura- } \\
\text { tion of hospital } \\
\text { stay) }\end{array}$} & \multirow[t]{2}{*}{-} & \multirow[t]{2}{*}{-} \\
\hline 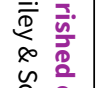 & & C: usual care & & & $23.3(5.9)$ & & & \\
\hline 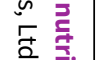 & \multirow{2}{*}{$\begin{array}{l}\text { Johansen } \\
2004\end{array}$} & I: nutrition team & - & \multirow{2}{*}{$\begin{array}{l}212 \text { (100) ESPEN } 2002 \\
\text { NRS (score > } 3 \text { nutrition- } \\
\text { ally at risk) }\end{array}$} & $21.2(0.5)$ & \multirow{2}{*}{$\begin{array}{l}\text { Duration of hos- } \\
\text { pital stay (dura- } \\
\text { tion of hospital } \\
\text { stay) }\end{array}$} & \multirow[t]{2}{*}{-} & \multirow[t]{2}{*}{-} \\
\hline 产 & & C: usual care & - & & $21.8(0.5)$ & & & \\
\hline 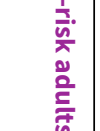 & \multirow[t]{2}{*}{ Kraft 2012} & I: ONS + telemedicine monitoring & - & \multirow{2}{*}{$\begin{array}{l}26(100) \text { weight loss }> \\
10 \% \text { in } 6 \text { months, } \mathrm{BMl}< \\
21 \mathrm{~kg} / \mathrm{m}^{2}, \text { albumin }<35 \\
\mathrm{~g} / \mathrm{L}\end{array}$} & $23.4(3.7)$ & \multirow[t]{2}{*}{$6 \mathrm{mo}(6 \mathrm{mo})$} & $\begin{array}{l}\text { Number of } \\
\text { medications: } \\
7.5 \text { (SD 4.2) }\end{array}$ & \multirow[t]{2}{*}{-} \\
\hline 尊 & & C: usual care & & & $23.4(4.5)$ & & $\begin{array}{l}\text { Number of } \\
\text { medications: } \\
8.2 \text { (SD 3.4) }\end{array}$ & \\
\hline & \multirow[t]{2}{*}{ Kretser 2003} & I: modified meals on wheels & 45 (44) white & $\begin{array}{l}97(96) \text { at risk or mal- } \\
\text { nourished according to } \\
\text { MNA }\end{array}$ & $\begin{array}{l}14(14 \%) \mathrm{BMI} \\
<18.5\end{array}$ & \multirow[t]{2}{*}{$26 w k(26 w k)$} & \multirow[t]{2}{*}{-} & \multirow{2}{*}{$\begin{array}{l}\text { A variety of self- } \\
\text { reported health } \\
\text { problems re- } \\
\text { ported }\end{array}$} \\
\hline & & C: traditional meals on wheels & 38 (58) white & $\begin{array}{l}95(95) \text { at risk or mal- } \\
\text { nourished }\end{array}$ & $\begin{array}{l}9(9 \%) \mathrm{BMI}< \\
18.5\end{array}$ & & & \\
\hline & Larsson 1990 & I: ONS plus normal hospital diet & - & (28.5) malnourished & - & $26 w k(26 w k)$ & - & - \\
\hline
\end{tabular}




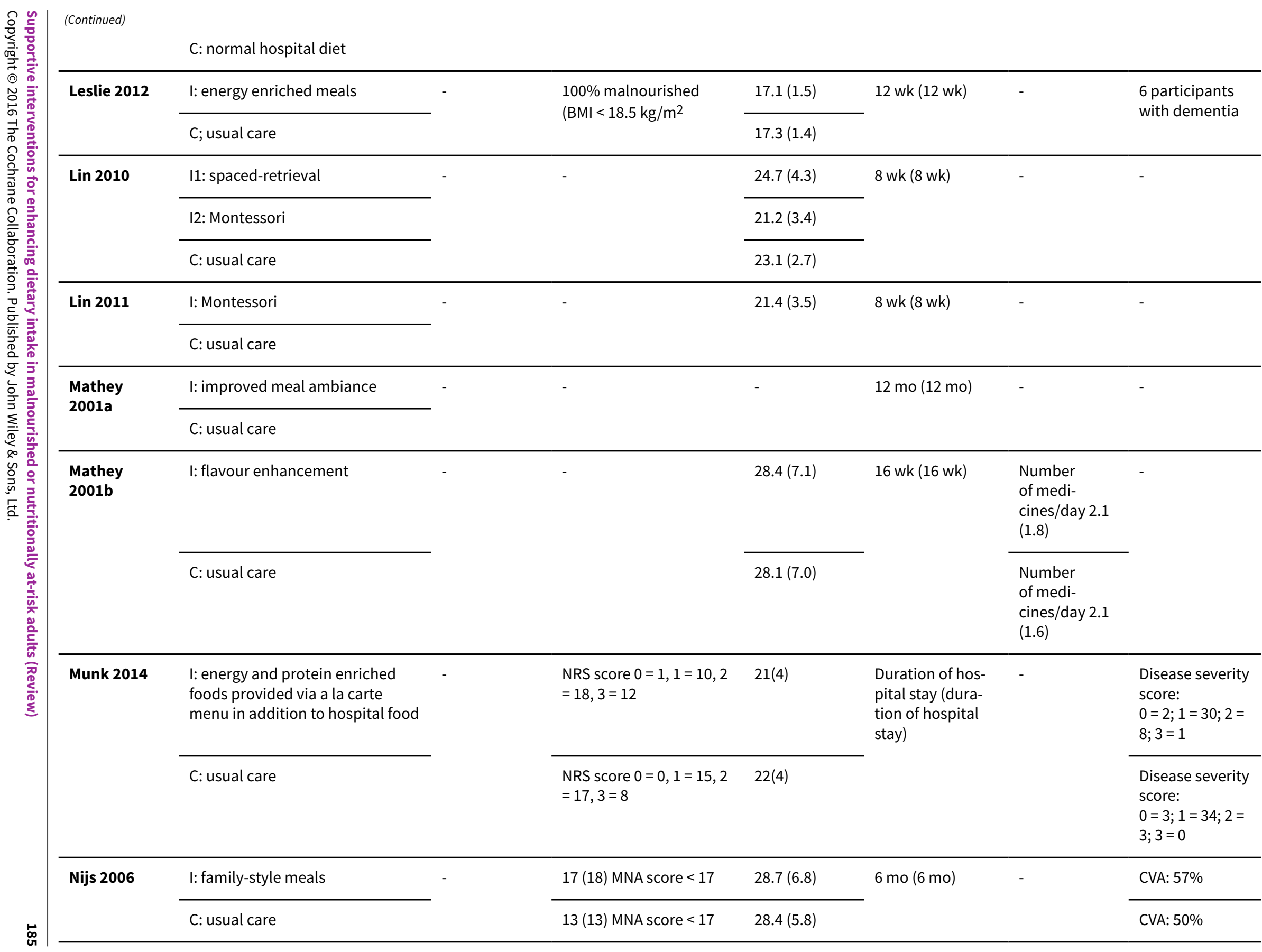




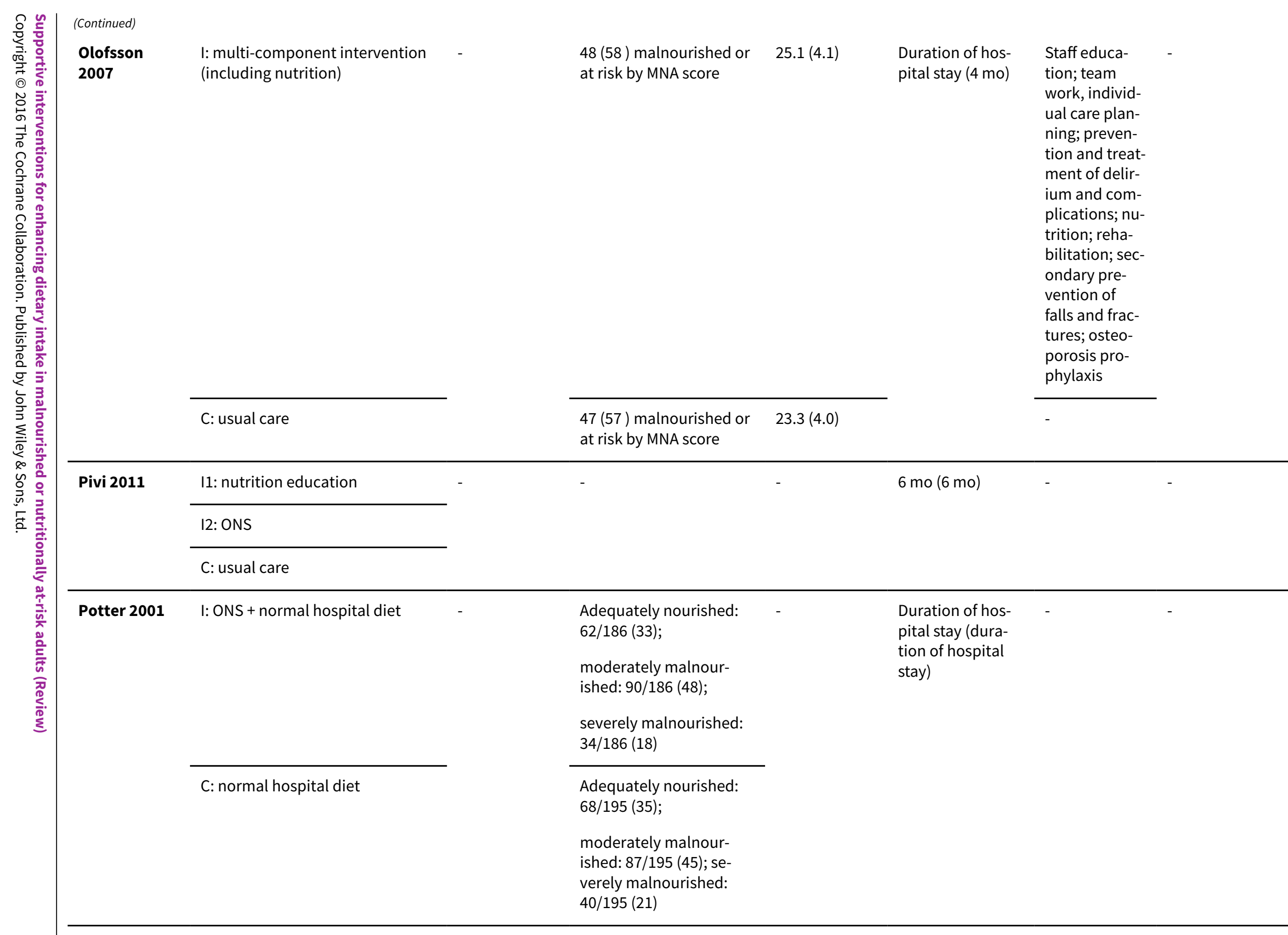




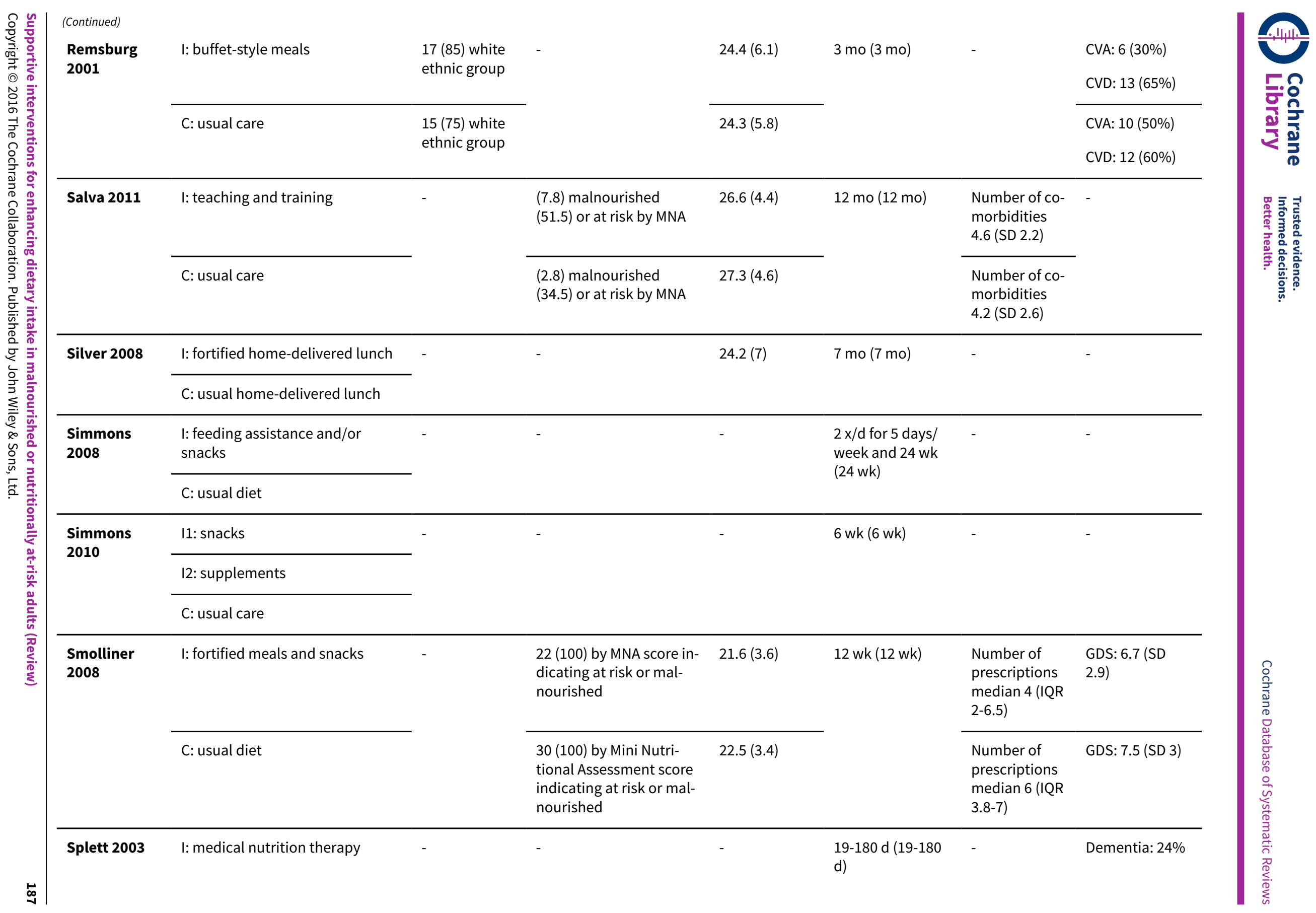




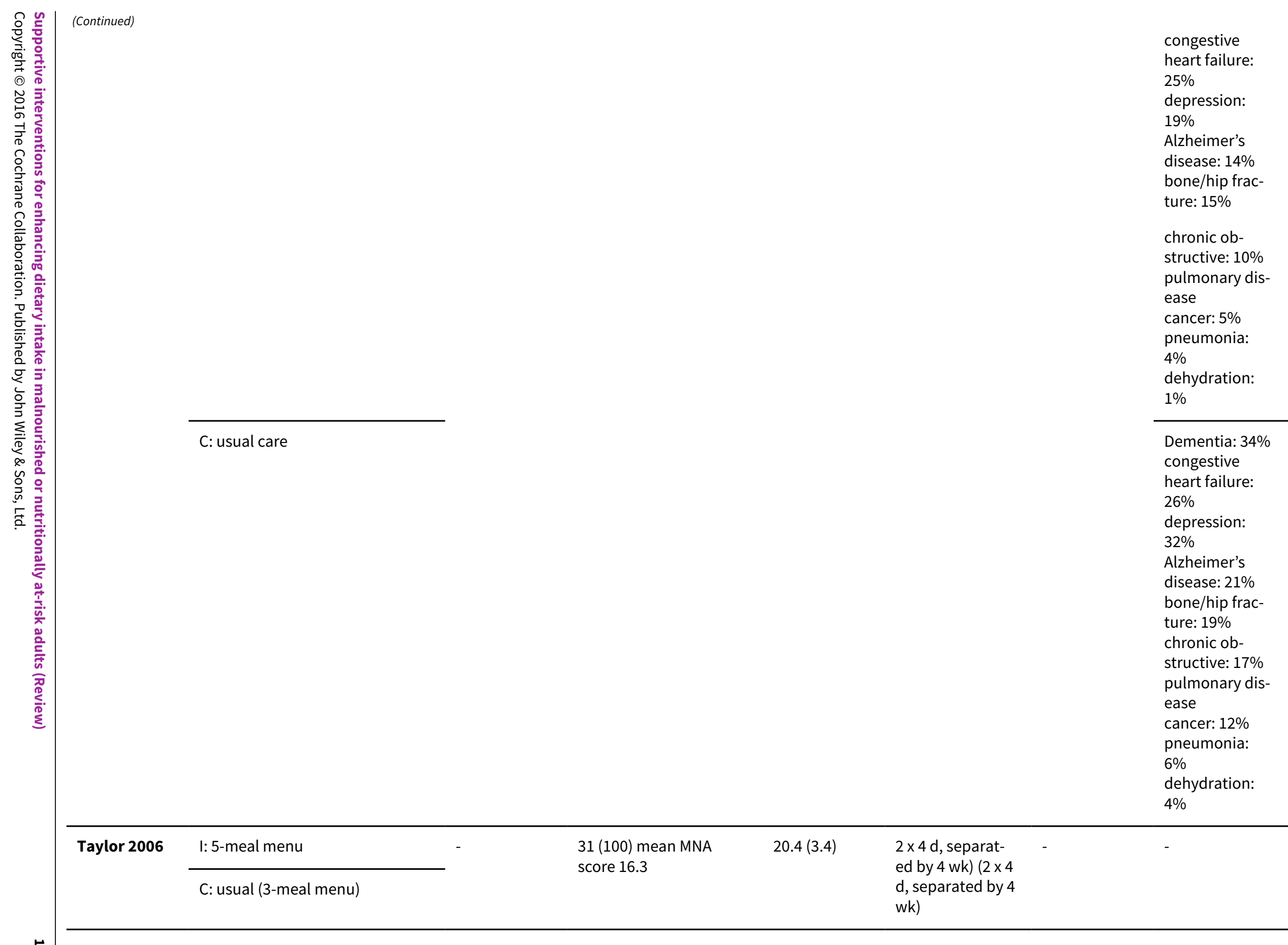




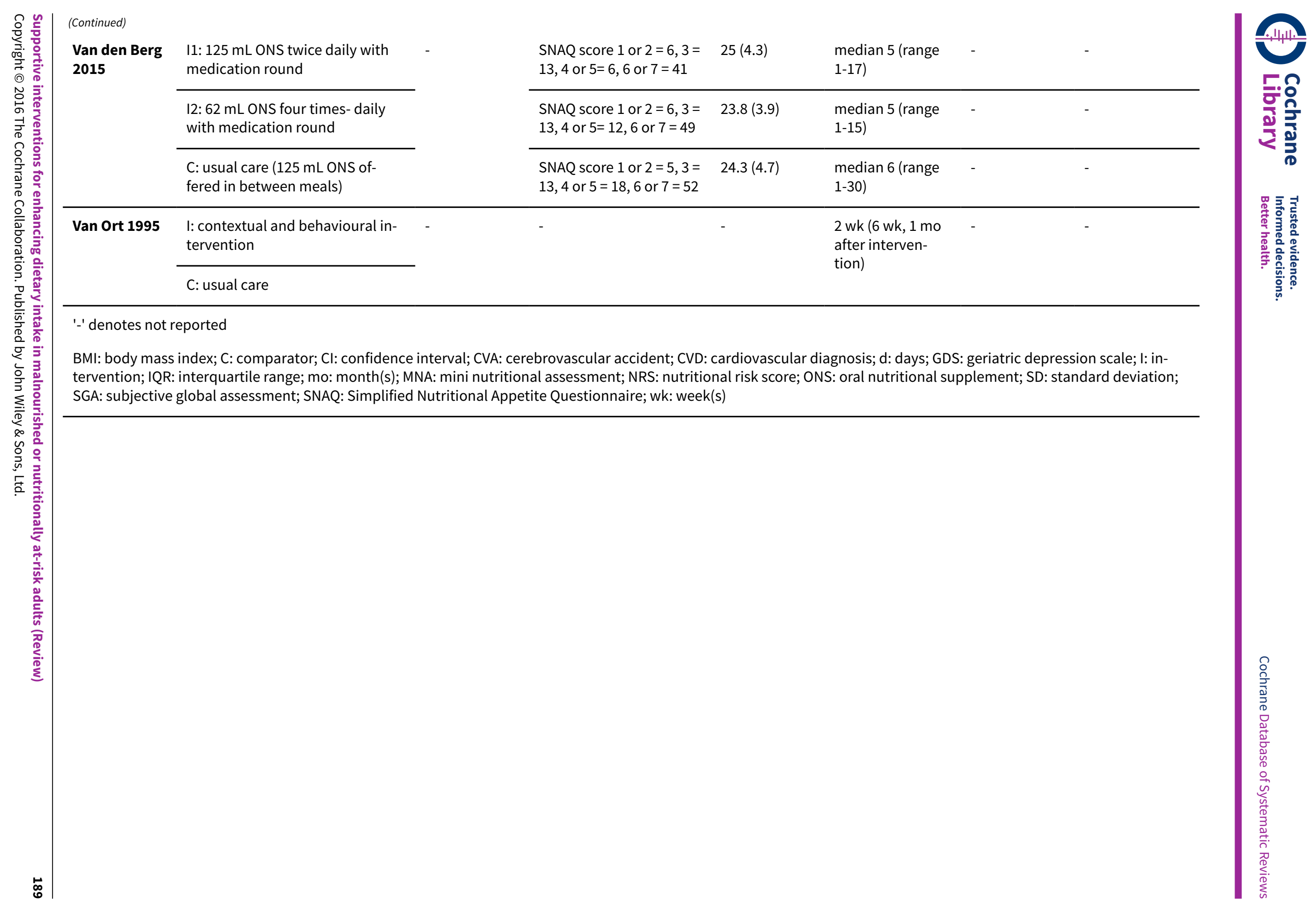


Appendix 6. Matrix of study endpoints (publications and trial documents)

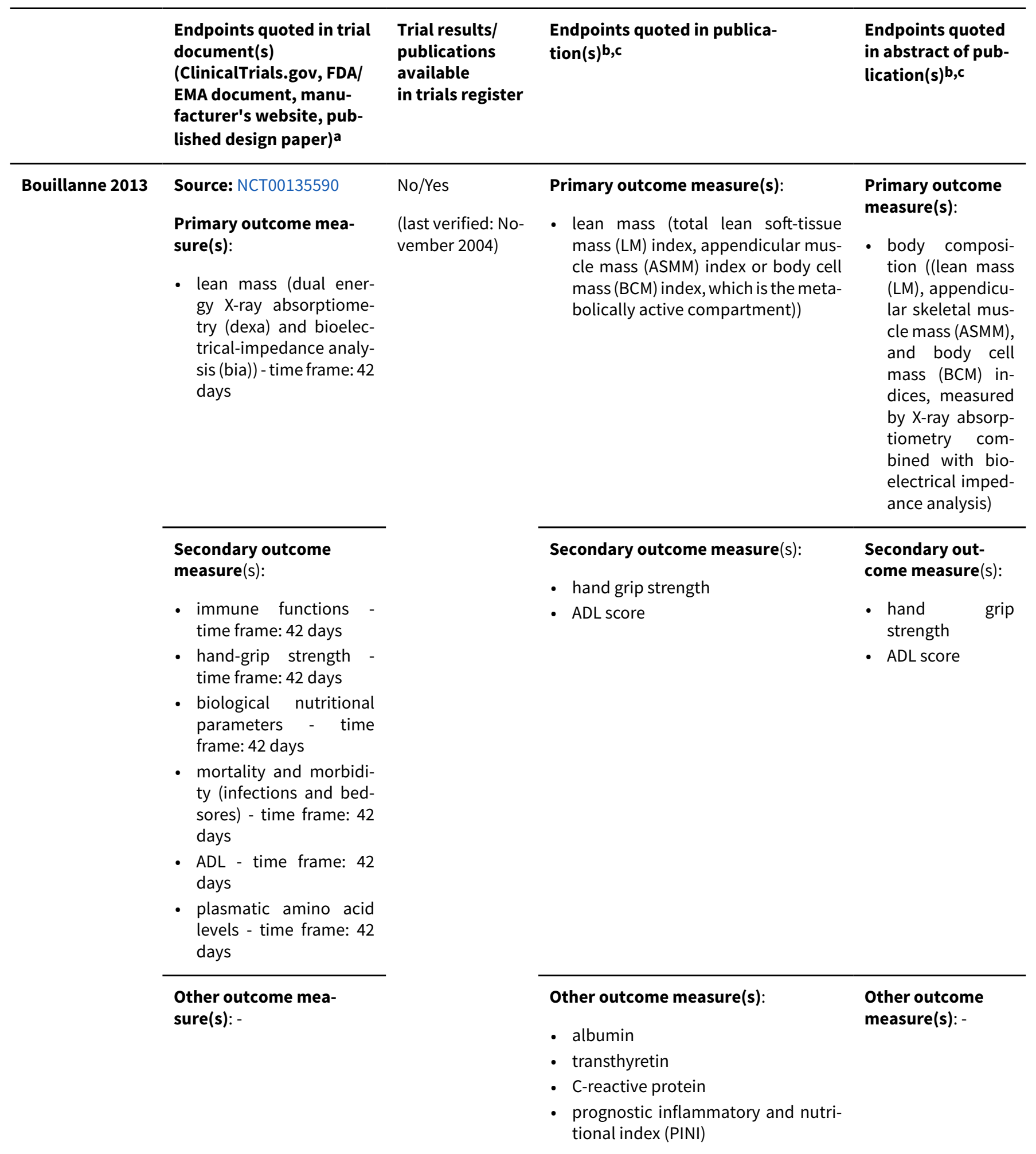

History of changes: 6 documented changes

\begin{tabular}{lllll}
\hline $\begin{array}{l}\text { Faxen-Irving } \\
2011\end{array}$ & Source: NCT01042340 No/Yes & Primary outcome measure(s): - & $\begin{array}{l}\text { Primary outcome } \\
\text { measure(s): - }\end{array}$
\end{tabular}


(Continued)

Primary outcome mea-
sure(s):
- to detect a significant
difference in energy in-
take of $48 \mathrm{kj} / 200 \mathrm{kcal}$ be-
tween the groups at $5 \%$
significance level and
with $80 \%$ power - time
frame: 5 days to 3 weeks
intervention

\section{Secondary outcome}

measure(s):

- effects on serum lipids and appetite - time frame: 5 days to 3 weeks treatment

Other outcome measure(s): - (last verified: December 2009)
Secondary outcome measure(s):

- acceptance and compliance of the concept by the participants at the ward
Secondary outcome measure(s): -

\section{Other outcome measure(s):}

- sample size calculation was performed: to detect a significant difference in energy intake of $200 \mathrm{kcal}$ between the groups at 5\% significance level and with $80 \%$ power, 27 participants in each group were needed. To allow for dropouts this was increased to 35 participants in each group

- nutritional assessment, by the Nutritional Risk Screening (NRS-2002) form: evaluation of BMI, weight loss, reduced dietary intake, age 70 and presence of severe illness and a sum score (0-7 points) was calculated

- biochemical indicators of nutritional status serum levels of albumin, transthyretin and insulin-like growth factor-1 (IGF-1)

- C-reactive protein (CRP)

- total cholesterol, high-density lipoprotein (HDL) and low-density lipoprotein (LDL) cholesterol, fasting serum triglyceride concentrations

- fatty acid (FA) profiles were measured in serum phospholipids

- function as determined by ADL according to the Katz ADL index

History of changes: 1 documented change

\begin{tabular}{lllll}
\hline Holyday 2012 & Source: NCT01179321 & No/No & Primary outcome measure(s): - & $\begin{array}{l}\text { Primary outcome } \\
\text { measure(s): }-\end{array}$ \\
& $\begin{array}{l}\text { Primary outcome mea- } \\
\text { sure(s): length of stay }\end{array}$ & $\begin{array}{l}\text { (last verified: } \\
\text { March 2006) }\end{array}$ & \\
\hline
\end{tabular}




\section{Secondary outcome measure(s): -}

Other outcome measure(s): -

\section{Secondary outcome measure(s): -}

Secondary outcome measure(s): -

\section{Other outcome measure(s):}

- pre-study power analysis based on the average length of stay (LOS) of the trial population $(11 \mathrm{~d})$ with 0.80 power using a test with significance of 0.05 , would require at least 50 participants in each group to detect a reduction in LOS of $20 \%$

- the number of participants seen by a clinical dietitian, number of consults per participant and total consultation time per participant was captured from the hospital's computerised dietitians' statistics system

- timeliness of intervention was counted as days between date of admission to the ward and the date seen by the clinical dietitian

- weight change over the course of admission was calculated from the weight on admission and the weight at discharge

- deaths during admission

- number of presentations to emergency and number of hospital readmissions

- cost of hospital admission, additional costs of a screening and nutritional intervention programme

\section{Other outcome} measure(s):

- length of stay LOS)

- weight change

- frequency of readmission to hospital

History of changes: 0 documented changes

\begin{tabular}{|c|c|c|c|c|}
\hline \multirow[t]{2}{*}{ Munk 2014} & $\begin{array}{l}\text { Source: NCT01415635 } \\
\text { Primary outcome mea- } \\
\text { sure(s): } \\
\text { - Percentage of partici- } \\
\text { pants reaching > 75\% } \\
\text { of their calculated ener- } \\
\text { gy and protein require- } \\
\text { ments }\end{array}$ & $\begin{array}{l}\text { No/No } \\
\text { (last verified: De- } \\
\text { cember 2012) }\end{array}$ & $\begin{array}{l}\text { Primary outcome measure(s): } \\
\text { - Percentage of participants reaching } \\
>75 \% \text { of their calculated energy and } \\
\text { protein requirements }\end{array}$ & $\begin{array}{l}\text { Primary outcome } \\
\text { measure(s): } \\
\text { - Percentage of } \\
\text { participants } \\
\text { reaching }>75 \% \\
\text { of their calculat- } \\
\text { ed energy and } \\
\text { protein require- } \\
\text { ments }\end{array}$ \\
\hline & $\begin{array}{l}\text { Secondary outcome } \\
\text { measure(s): } \\
\text { - handgrip strength } \\
\text { - daily energy and pro- } \\
\text { tein intake } \\
\text { - use of tube feeding } \\
\text { - use of parenteral nutri- } \\
\text { tion } \\
\text { - length of stay }\end{array}$ & & $\begin{array}{l}\text { Secondary outcome measure(s): } \\
\text { - Mean daily energy and protein in- } \\
\text { take } \\
\text { - body weight } \\
\text { - handgrip strength } \\
\text { - Length of stay } \\
\text { - number of participants receiving en- } \\
\text { teral or parenteral feeding }\end{array}$ & $\begin{array}{l}\text { Secondary out- } \\
\text { come measure(s): } \\
\text { - Mean daily ener- } \\
\text { gy and protein } \\
\text { intake } \\
\text { - body weight } \\
\text { - handgrip } \\
\text { strength } \\
\text { - Length of stay }\end{array}$ \\
\hline
\end{tabular}


(Continued)

Other outcome mea-

sure(s): none provided
Other outcome measure(s): number

of participants receiving ONS
Other outcome measure(s): -

History of changes: 1 documented change

\begin{tabular}{|c|c|c|c|c|}
\hline \multirow[t]{3}{*}{ Nijs 2006} & $\begin{array}{l}\text { Source: NCT00114582 } \\
\text { Primary outcome mea- } \\
\text { sure: } \\
\text { - nutritional status, qual- } \\
\text { ity of life, physical per- } \\
\text { formance }\end{array}$ & $\begin{array}{l}\text { No/Yes } \\
\text { (last verified: } \\
\text { February 2009) }\end{array}$ & $\begin{array}{l}\text { Primary outcome measure(s): } \\
\text { - quality of life }\end{array}$ & $\begin{array}{l}\text { Primary outcome } \\
\text { measure(s): - }\end{array}$ \\
\hline & $\begin{array}{l}\text { Secondary outcome } \\
\text { measure(s): - }\end{array}$ & & Secondary outcome measure(s): - & $\begin{array}{l}\text { Secondary out- } \\
\text { come measure(s): - }\end{array}$ \\
\hline & $\begin{array}{l}\text { Other outcome mea- } \\
\text { sure(s): - }\end{array}$ & & $\begin{array}{l}\text { Other outcome measure(s): } \\
\text { - physical performance } \\
\text { - body weight } \\
\text { - energy intake }\end{array}$ & $\begin{array}{l}\text { Other outcome } \\
\text { measure(s): } \\
\text { - quality of life } \\
\text { (perceived safe- } \\
\text { ty; autonomy; } \\
\text { and sensory, } \\
\text { physical, and } \\
\text { psychosocial } \\
\text { functioning) } \\
\text { - gross and fine } \\
\text { motor function } \\
\text { - body weight }\end{array}$ \\
\hline
\end{tabular}

History of changes: 4 documented changes

\begin{tabular}{|c|c|c|c|c|}
\hline \multirow[t]{2}{*}{ Salva 2011} & $\begin{array}{l}\text { Source: NCT00479843 } \\
\text { Primary outcome mea- } \\
\text { sure(s): } \\
\text { - evaluation of the effec- } \\
\text { tiveness of the interven- } \\
\text { tion - the main eval- } \\
\text { uation criteria which } \\
\text { would allow the effec- } \\
\text { tiveness of this inter- } \\
\text { vention to be evaluat- } \\
\text { ed were the reduction } \\
\text { in the loss of autonomy } \\
\text { measured by the ADL/ } \\
\text { iADL scale - time frame: } \\
\text { baseline, } 6 \text { months, } 12 \\
\text { months }\end{array}$ & $\begin{array}{l}\text { No/Yes } \\
\text { (last verified: } \\
\text { January 2014) }\end{array}$ & Primary outcome measure(s): - & $\begin{array}{l}\text { Primary outcome } \\
\text { measure(s): } \\
\text { - main outcome } \\
\text { measure was the } \\
\text { reduction in the } \\
\text { loss of auton- } \\
\text { omy ((ADL/IADL) } \\
\text { scales) assessed } \\
\text { at } 6 \text { and } 12 \\
\text { months }\end{array}$ \\
\hline & $\begin{array}{l}\text { Secondary outcome } \\
\text { measure(s): } \\
\text { - improvement in the } \\
\text { participant's state of } \\
\text { nutrition - reducing the } \\
\text { burden on carers with } \\
\text { the Zarit scale }\end{array}$ & & $\begin{array}{l}\text { Secondary outcome measure(s): } \\
\text { - improvement in nutritional state of } \\
\text { the participant evaluated by their } \\
\text { change in weight, BMI and MNA } \\
\text { - reduction in burden on caregiver } \\
\text { (ZARIT scale) }\end{array}$ & $\begin{array}{l}\text { Secondary out- } \\
\text { come measure(s): } \\
\text { - improvement in } \\
\text { nutritional sta- } \\
\text { tus (Mini Nu- } \\
\text { tritional Assess- } \\
\text { ment (MNA), }\end{array}$ \\
\hline
\end{tabular}


(Continued)

- evaluation of the use of healthcare and social resources with the RUD scale

- improvement of medical practice regarding nutrition

(time frame: baseline, 6 months, 12 months)

Other outcome measure(s): -
- reduction in the use of healthcare and social resources (RUD scale)
BMI, and weight changes)

- caregiver burden (Zarit scale)
Other outcome measure(s):

- our primary hypothesis was that participants in the intervention group would achieve a lower level of dependency compared with participants in the usual care-control group at 12 months. We considered a significant benefit in the intervention group to be a reduction of $30 \%$ in the proportion of participants who lost more than 0.5 points according to the ADL score (loss of autonomy) over one year

History of changes: 2 documented changes

\begin{tabular}{|c|c|c|c|c|}
\hline \multirow[t]{3}{*}{$\begin{array}{l}\text { Van den Berg } \\
2015\end{array}$} & $\begin{array}{l}\text { Source: NTR2535 } \\
\text { Primary outcome mea- } \\
\text { sure(s): proportion of par- } \\
\text { ticipants who received } \\
\text { their treatment goal. The } \\
\text { treatment goal was to re- } \\
\text { ceive at least } 75 \% \text { of the } \\
\text { prescribed volume of ONS } \\
\text { during admission }\end{array}$ & $\begin{array}{l}\text { No (last verified } \\
19 \text { Nov 2010) }\end{array}$ & $\begin{array}{l}\text { Primary outcome measure(s):the } \\
\text { percentage of participants who } \\
\text { reached the treatment objective of at } \\
\text { least } 75 \% \text { of the prescribed volume of } \\
\text { ONS during admission }\end{array}$ & $\begin{array}{l}\text { Primary outcome } \\
\text { measure(s): } \\
\text { The percentage of } \\
\text { participants who } \\
\text { consumed at least } \\
75 \% \text { of the pre- } \\
\text { scribed volume of } \\
\text { ONS }\end{array}$ \\
\hline & $\begin{array}{l}\text { Secondary outcome } \\
\text { measure(s): } \\
\text { intake (mL of ONS) (nurses } \\
\text { and food assistants read } \\
\text { the amount of ONS left in } \\
\text { the bottle) }\end{array}$ & & $\begin{array}{l}\text { Secondary outcome measure }(\mathrm{s}) \text { : } \\
\text { Mean intake of ONS per day in } \mathrm{mL} \text { and } \\
\text { energy and protein }\end{array}$ & Not stated \\
\hline & $\begin{array}{l}\text { Other outcome mea- } \\
\text { sure(s):- }\end{array}$ & & $\begin{array}{l}\text { Other outcome measure(s): length of } \\
\text { hospital stay, hospital readmissions, } \\
\text { time to intervention, duration of inter- } \\
\text { vention, mortality }\end{array}$ & $\begin{array}{l}\text { Median time of tak- } \\
\text { ing ONS }\end{array}$ \\
\hline
\end{tabular}

History of changes: No documented changes

\footnotetext{
'-' denotes not reported

aTrial document(s) refers to all available information from published design papers and sources other than regular publications (e.g. FDA/EMA documents, manufacturer's websites, trials registers)

bPublication(s) refers to trial information published in scientific journals (primary reference, duplicate publications, companion documents or multiple reports of a primary trial)
} 
(Continued)

cOther outcome measures refer to all outcomes not specified as primary or secondary outcome measures

ADL: activities of daily living; BMI: body mass index; EMA: European Medicines Agency; FDA: Food and Drug Administration (US); mo: month(s); N/A: not applicable; N/T: no trial document available; yr: year(s); wk: week(s); ONS oral nutritional supplement

Appendix 7. High risk of outcome reporting bias according to ORBIT classification

\begin{tabular}{|c|c|c|c|c|c|}
\hline & Outcome & $\begin{array}{l}\text { High risk of } \\
\text { bias } \\
\text { (category A)a }\end{array}$ & $\begin{array}{l}\text { High risk of } \\
\text { bias } \\
\text { (category } \\
\text { D)b }\end{array}$ & $\begin{array}{l}\text { High risk of } \\
\text { bias } \\
\text { (category E)c }\end{array}$ & $\begin{array}{l}\text { High risk of } \\
\text { bias } \\
\text { (category } \\
\text { G)d }\end{array}$ \\
\hline \multirow[t]{3}{*}{ Barton 2000} & Energy intake & Yes & & & \\
\hline & Food wastage & Yes & & & \\
\hline & Protein intake & Yes & & & \\
\hline Beck 2002 & $\mathrm{~N} / \mathrm{D}$ & & & & \\
\hline $\begin{array}{l}\text { Bouillanne } \\
2013\end{array}$ & N/D & & & & \\
\hline \multirow{3}{*}{$\begin{array}{l}\text { Bourdel-Mar- } \\
\text { chasson } 2000\end{array}$} & Energy intake & Yes & & & \\
\hline & Incidence of death & Yes & & & \\
\hline & Pressure ulcer developments & & & $\begin{array}{l}\text { Yes ( } 40 \% \text { in } \\
\text { intervention } \\
\text { group, } 48 \% \\
\text { in control; no } \\
\text { further analy- } \\
\text { sis) }\end{array}$ & \\
\hline \multirow{2}{*}{$\begin{array}{l}\text { Castellanos } \\
2009\end{array}$} & 3 meal energy intake & & Yes & & \\
\hline & 3 meal protein intake & & Yes & & \\
\hline Chang 2005 & $\mathrm{~N} / \mathrm{D}$ & & & & \\
\hline \multirow[t]{6}{*}{ Dennis 2005} & Death or poor outcome & Yes & & & \\
\hline & Death & Yes & & & \\
\hline & $\begin{array}{l}\text { Complications: pneumonia, UTI, pressure } \\
\text { sores }\end{array}$ & Yes & & & \\
\hline & Length of stay & Yes & & & \\
\hline & Discharge destination & Yes & & & \\
\hline & EUROQOL & Yes & & & \\
\hline Duncan 2006 & $\mathrm{~N} / \mathrm{D}$ & & & & \\
\hline
\end{tabular}


(Continued)

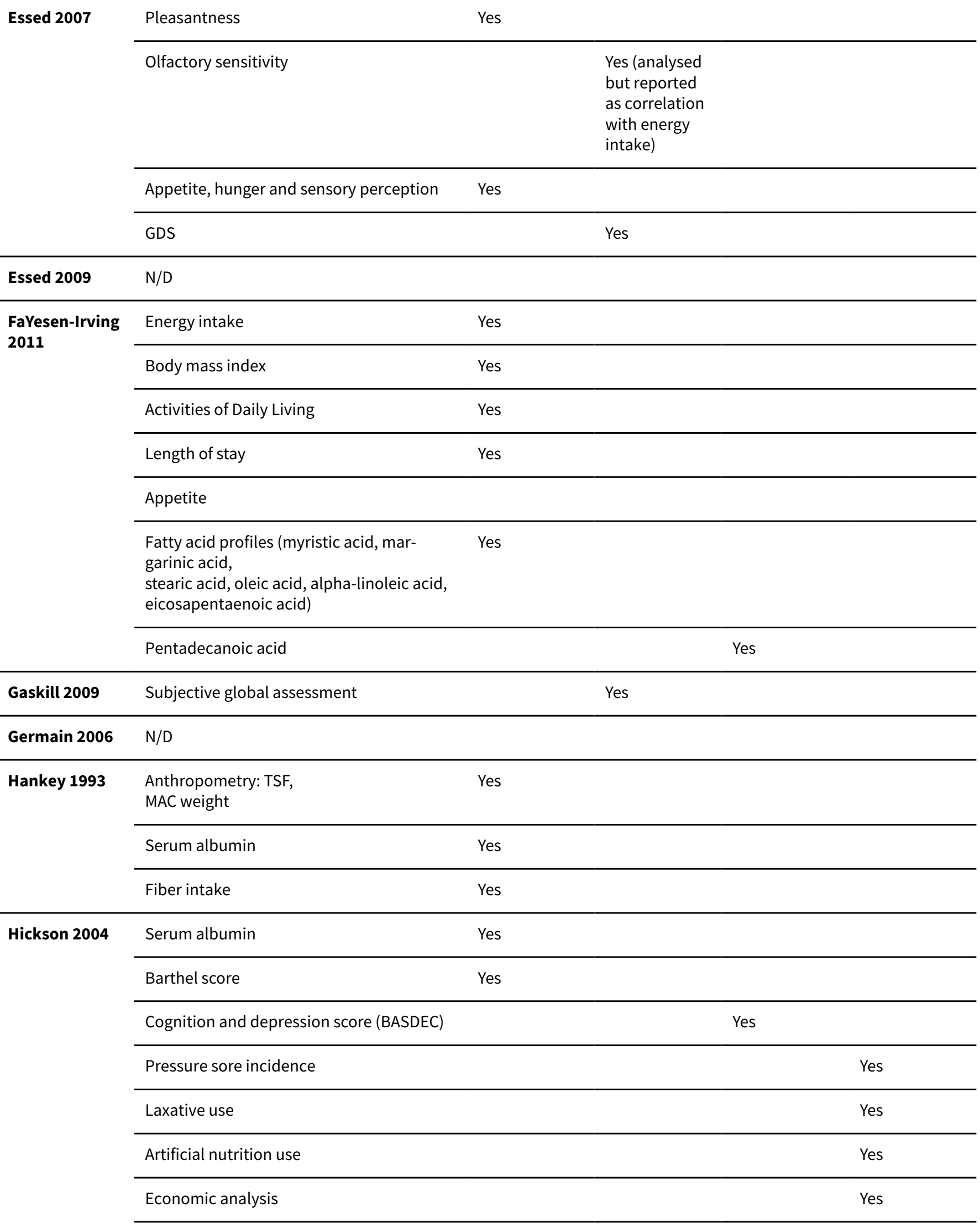


(Continued)

Dietary intake

In-hospital mortality

Grip strength

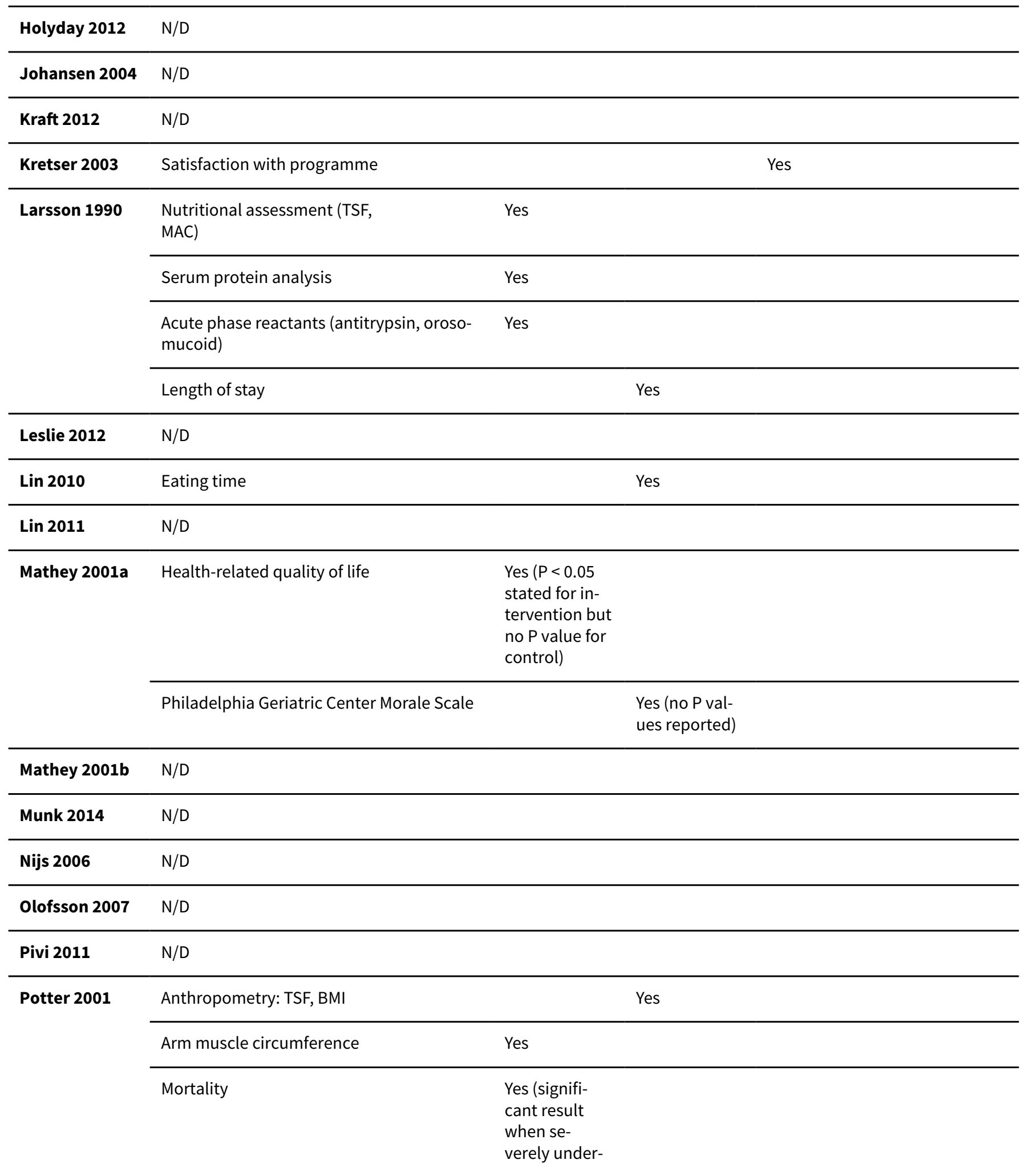


(Continued)

\begin{tabular}{ll} 
& $\begin{array}{l}\text { nourished } \\
\text { analysed in } \\
\text { isolation) }\end{array}$ \\
\hline Functional recovery (Barthel ADL index) & $\begin{array}{l}\text { Yes (signifi- } \\
\text { cant result } \\
\text { when se- } \\
\text { verely under- } \\
\text { nourished } \\
\text { analysed in } \\
\text { isolation) }\end{array}$ \\
\hline Discharge placement & Yes \\
\hline Length of hospital stay & Yes
\end{tabular}

Remsburg N/D
2001

\begin{tabular}{lll}
\hline Salva 2012 & $\begin{array}{l}\text { Health and social care costs (Resource Util- } \\
\text { isation } \\
\text { in Dementia (RUD) scale) }\end{array}$
\end{tabular}

Silver $2008 \quad$ Confounding effect of age, sex and BMI on Yes

treatment order, total energy, energy den-

sity and macronutrients

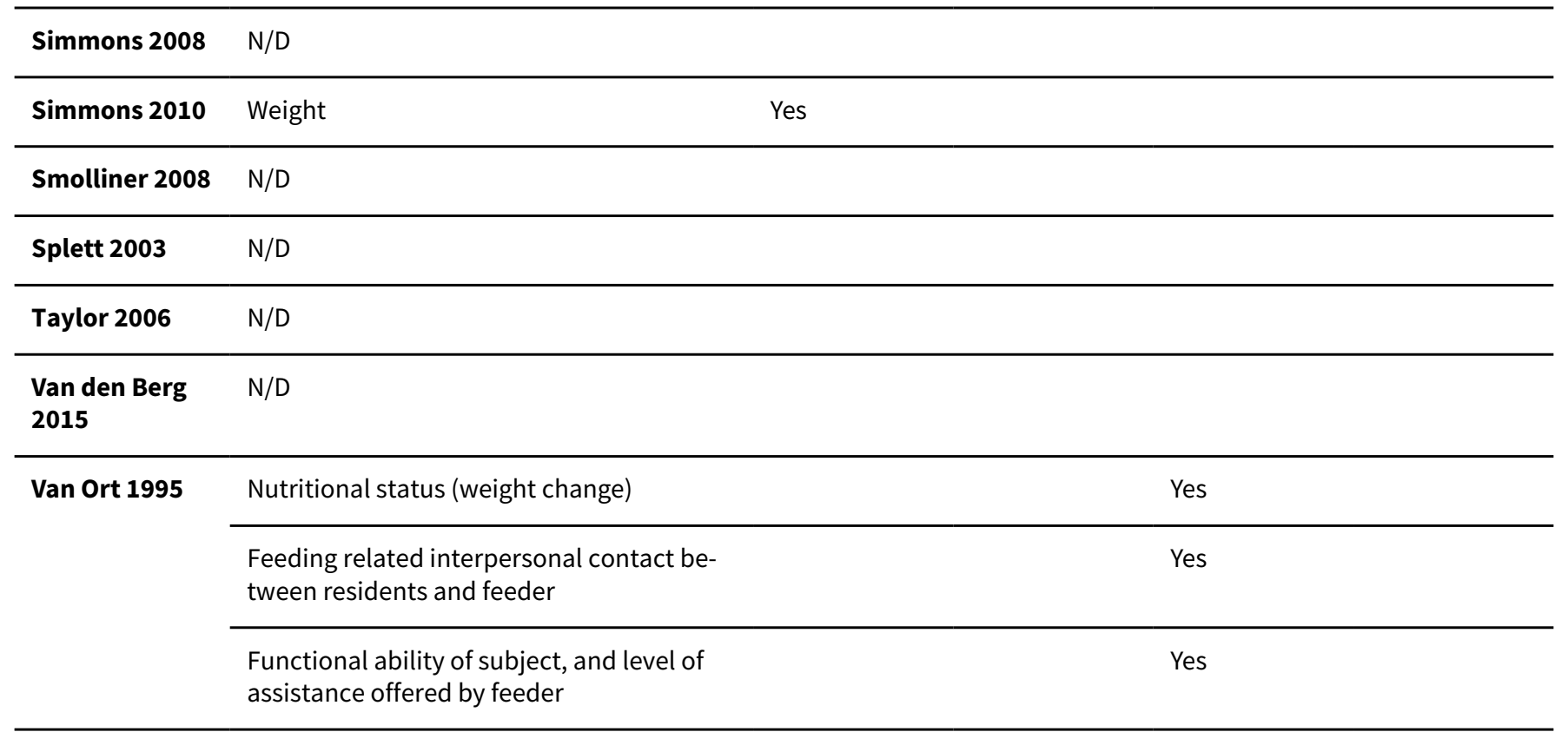

aclear that outcome was measured and analysed; trial report states that outcome was analysed but only reports that result was not significant

(Classification 'A', table 2, Kirkham 2010)

bClear that outcome was measured and analysed; trial report states that outcome was analysed but no results reported (Classification 'D', table 2, Kirkham 2010)

'Clear that outcome was measured; clear that outcome was measured but not necessarily analysed; judgement says likely to have been analysed but not reported because of non-significant results

(Classification 'E', table 2, Kirkham 2010) 
(Continued)

dunclear whether the outcome was measured; not mentioned but clinical judgement says likely to have been measured and analysed but not reported on the basis of non-significant results

(Classification 'G', table 2, Kirkham 2010)

ADL: activities of daily living; BMI: body mass index; EuroQol: European Quality of Life Scale; GDS: geriatric depression scale; mo: months; N/D: none detected; ORBIT: Outcome Reporting Bias In Trials; TSF: triceps skinfold thickness

\section{Appendix 8. Definition of endpoint measurement (I)}

\begin{tabular}{|c|c|c|c|c|c|}
\hline & Nutritional intake & $\begin{array}{l}\text { Health-relat- } \\
\text { ed quality of } \\
\text { life/patient } \\
\text { satisfaction }\end{array}$ & Mortality & $\begin{array}{l}\text { Morbidi- } \\
\text { ty/complica- } \\
\text { tions }\end{array}$ & Nutritional status \\
\hline Barton 2000 & $\begin{array}{l}\text { Energy intake (kcal), protein intake } \\
(\mathrm{g}), \text { food wastage }(\%)\end{array}$ & - & - & - & - \\
\hline Beck 2002 & Energy intake (MJ) & - & - & - & Weight (kg) \\
\hline Bouillanne 2013 & - & - & Yes & Infections & Body composition \\
\hline $\begin{array}{l}\text { Bourdel-Mar- } \\
\text { chasson } 2000\end{array}$ & $\begin{array}{l}\text { Energy intake (kcal), protein intake } \\
\text { (g) }\end{array}$ & - & Yes & - & - \\
\hline Brouillette 1991 & $\begin{array}{l}\text { Energy intake (kcal), \% food con- } \\
\text { sumed }\end{array}$ & - & Yes & - & - \\
\hline $\begin{array}{l}\text { Castellanos } \\
2009\end{array}$ & $\begin{array}{l}\text { Energy intake (kcal), protein intake } \\
\text { (g) }\end{array}$ & - & & - & - \\
\hline Chang 2005 & $\%$ meal eaten & - & - & - & - \\
\hline Dennis 2005 & - & $\begin{array}{l}\text { Quality of life } \\
\text { (EUROQoL) }\end{array}$ & Yes & $\begin{array}{l}\text { Incidence of } \\
\text { pneumonia, } \\
\text { UTI and pres- } \\
\text { sure sores }\end{array}$ & - \\
\hline Duncan 2006 & Dietary intake records on day 3-6 & - & - & $\begin{array}{l}\text { Records of } \\
\text { medical and } \\
\text { surgical com- } \\
\text { plications }\end{array}$ & $\begin{array}{l}\text { Weight, MAMC, TSF, } \\
\text { HGS }\end{array}$ \\
\hline Essed 2007 & $\begin{array}{l}\text { Energy intake }(\mathrm{kJ}) \text {, protein, fat and } \\
\mathrm{CHO}(\mathrm{g})\end{array}$ & - & - & - & $\begin{array}{l}\text { Weight }(\mathrm{kg}), \mathrm{BMI} \\
\text { body composition }\end{array}$ \\
\hline Essed 2009 & Energy intake (kJ) & - & - & - & - \\
\hline $\begin{array}{l}\text { Faxen-Irving } \\
2011\end{array}$ & $\begin{array}{l}\text { Energy intake (kcal/kg body weight/ } \\
\text { day) }\end{array}$ & - & Yes & - & $\begin{array}{l}\text { Weight, appetite, } \\
\text { BMI }\end{array}$ \\
\hline Gaskill 2009 & SGA & - & - & - & - \\
\hline Germain 2006 & $\begin{array}{l}\text { Dietary intake, energy (kcal), other } \\
\text { nutrients }(\mathrm{g} / \mathrm{mg})\end{array}$ & - & Yes & - & Weight, BMI \\
\hline
\end{tabular}


(Continued)

\begin{tabular}{|c|c|c|c|c|c|}
\hline Hankey 1993 & $\begin{array}{l}\text { Energy intake (kj/24hours), protein } \\
\text { intake (g/24hours) }\end{array}$ & - & - & - & $\begin{array}{l}\text { Weight, TSF, MAC, } \\
\text { AMC, serum albu- } \\
\text { min }\end{array}$ \\
\hline Hickson 2004 & Energy intake $(\mathrm{J})$, protein $(\mathrm{g})$ & EQ-5D & Yes & $\begin{array}{l}\text { Antibiotics } \\
\text { prescribed } \\
(\mathrm{N}) \text {, days on } \\
\text { antibiotics }\end{array}$ & $\begin{array}{l}\text { Weight, BMI, MAC, } \\
\text { TSF, MAMC, }\end{array}$ \\
\hline Holyday 2012 & - & - & Yes & - & Weight \\
\hline Johansen 2004 & $\begin{array}{l}\text { Energy intake ( } \mathrm{kJ} / \mathrm{kg} \text { and } \% \text { require- } \\
\text { ments), protein intake ( } \mathrm{g} / \mathrm{kg} \text { and } \% \\
\text { requirements) }\end{array}$ & SF-36 & Yes & $\begin{array}{l}\text { Infectious and } \\
\text { other compli- } \\
\text { cations grad- } \\
\text { ed into major } \\
\text { and minor (us- } \\
\text { ing Buzby et al } \\
1988 \text { and CDC } \\
\text { definitions) }\end{array}$ & Weight change (kg) \\
\hline
\end{tabular}

\begin{tabular}{|c|c|c|c|c|c|}
\hline Kraft 2012 & - & - & - & - & Weight change (kg) \\
\hline Kretser 2003 & - & - & Yes & - & $\begin{array}{l}\text { Weight, weight } \\
\text { change (lb) }\end{array}$ \\
\hline Larsson 1990 & $\begin{array}{l}\text { Encompassed in the Modified Nor- } \\
\text { ton Scale }\end{array}$ & - & Yes & - & $\begin{array}{l}\text { Weight index, TSF, } \\
\text { MAC, AMC }\end{array}$ \\
\hline Leslie 2012 & $\begin{array}{l}\text { Dietary intake, } 3 \text { day weighed } \\
\text { records }\end{array}$ & - & Yes & - & $\begin{array}{l}\text { Weight change, } \\
\text { change in BMI \& } \\
\text { MUAC }\end{array}$ \\
\hline Lin 2010 & Eating amount (unit unclear) & - & No & - & MNA and BMI \\
\hline Lin 2011 & - & - & No & - & MNA and BMI \\
\hline Mathey 2001a & Macro- and micronutrient intakes & SIP, PGCMS & Yes & No & Weight \\
\hline Mathey 2001b & Energy intake & - & No & no & Weight \\
\hline Munk 2014 & $\begin{array}{l}\text { Percent of participants meeting }> \\
75 \% \text { of their energy and protein re- } \\
\text { quirements. Mean daily energy and } \\
\text { protein intake }\end{array}$ & - & Yes & - & Weight \\
\hline Nijs 2006 & $\begin{array}{l}\text { Energy intake (kcal), macronutrient } \\
\text { (g) }\end{array}$ & $\begin{array}{l}\text { Dutch QOL } \\
\text { nursing home } \\
\text { residents } \\
\text { questionnaire }\end{array}$ & Yes & - & $\begin{array}{l}\text { Weight }(\mathrm{kg} \text {, calf cir- } \\
\text { cumference }(\mathrm{cm}) \text {, } \\
\text { MAC }(\mathrm{cm}), \text { MNA } \\
\text { score }\end{array}$ \\
\hline Olofsson 2007 & - & - & Yes & $\begin{array}{l}\text { Infectious and } \\
\text { non-infectious } \\
\text { complications } \\
\text { during hospi- } \\
\text { tal stay }\end{array}$ & Weight, BMI, MNA \\
\hline Pivi 2011 & - & - & Yes & - & $\begin{array}{l}\text { Weight, BMI, MAC, } \\
\text { MAMC, TSF }\end{array}$ \\
\hline
\end{tabular}


(Continued)

\begin{tabular}{|c|c|c|c|c|c|}
\hline Potter 2001 & Total energy intake (kcal) & - & Yes & - & $\begin{array}{l}\text { Weight, AMC, TSF, } \\
\text { BMI }\end{array}$ \\
\hline Remsburg 2001 & - & - & Yes & - & Weight (kg) \\
\hline Salva 2011 & - & - & Yes & - & $\begin{array}{l}\text { Weight (kg), BMI, } \\
\text { MNA }\end{array}$ \\
\hline Silver 2008 & $\begin{array}{l}\text { Total energy (kcal), energy densi- } \\
\text { ty (kcal/g), macronutrient's (g), mi- } \\
\text { cronutrients }\end{array}$ & - & - & - & - \\
\hline Simmons 2008 & Total energy (kcal) & - & - & - & Weight (lb), BMI \\
\hline Simmons 2010 & Energy intake (kcal) & - & Yes & - & Weight (lb) \\
\hline Smolliner 2008 & $\begin{array}{l}\text { Energy (kcal and kcal/kg body } \\
\text { weight), protein ( } \mathrm{g} \text { and } \mathrm{g} / \mathrm{kg} \text { body } \\
\text { weight) }\end{array}$ & - & Yes & - & $\begin{array}{l}\text { Weight, BMI, MNA } \\
\text { score, fat-free mass }\end{array}$ \\
\hline Splett 2003 & - & - & Yes & - & Weight \\
\hline Taylor 2006 & $\begin{array}{l}\text { Energy intake (kcal/day), fluid in- } \\
\text { take (mL/day) }\end{array}$ & - & - & - & - \\
\hline $\begin{array}{l}\text { Van den Berg } \\
2015\end{array}$ & Energy intake from ONS (kcal/day) & - & Yes & & \\
\hline Van Ort & - & - & - & - & Weight \\
\hline
\end{tabular}

ADL: activities of daily living; AMC: arm muscle circumference; BMI: body mass index; CDC: Centre for Disease Control; CDR: clinical dementia rating scale; CHO: carbohydrate; d: day; EQ-5D/EuroQol: European Quality of Life Scale; HGS: handgrip strength; iADL: instrumental Activities of Daily Living; MAC: mid-arm circumference; MAMC: mid-arm muscle circumference; MJ: mega joules; MMSE: Mini Mental State Examination; MNA: Mini Nutritional Assessment; NPIQ: Neuropyschiatric Inventory Question; PGCMS: Philadelphia Geriatric Centre Morale Scale; QOL: quality of life; RUD: reduction in use of health and social care scale; SF-36: Short Form - 36; SGA: subjective global assessment; SIP: sickness impact profile; TSF: triceps skinfold thickness

\section{Appendix 9. Definition of endpoint measurement (II)}

\begin{tabular}{lllll}
\hline & Functional status & $\begin{array}{l}\text { Clinical func- } \\
\text { tion }\end{array}$ & $\begin{array}{l}\text { Hospitalisation/ } \\
\text { institutionalisation }\end{array}$ & $\begin{array}{l}\text { Severe/seri- } \\
\text { ous adverse } \\
\text { events }\end{array}$ \\
\hline Barton 2000 & - & $\begin{array}{l}\text { Economic } \\
\text { costs }\end{array}$ & - \\
\hline Beck 2002 & - & - & - & - \\
\hline Bouillanne 2013 & $\begin{array}{l}\text { Handgrip strength, ADL } \\
\text { score }\end{array}$ & $\begin{array}{l}\text { Biochemical } \\
\text { data }\end{array}$ & - & - \\
\hline $\begin{array}{l}\text { Bourdel-Marchasson } \\
\mathbf{2 0 0 0}\end{array}$ & - & $\begin{array}{l}\text { Pressure ulcer } \\
\text { development }\end{array}$ & - \\
\hline
\end{tabular}


(Continued)

Brouillette 1991

\section{Castellanos 2009}

\begin{tabular}{lllll}
\hline Chang 2005 & - & - & - & - \\
\hline Dennis 2005 & - & - & Discharge destination & - \\
\hline Duncan 2006 & - & - & $\begin{array}{l}\text { Length of stay in acute } \\
\text { unit and in hospital } \\
\text { (days) }\end{array}$ & - \\
\hline Essed 2007 & - & - & - \\
\hline Essed 2009 & - & - & - & - \\
\hline Faxen-Irving 2011 & - & $\begin{array}{l}\text { Serum/plas- } \\
\text { ma proteins, } \\
\text { serum lipids, } \\
\text { fatty acid pro- } \\
\text { files, ADLs }\end{array}$ \\
\hline
\end{tabular}

\begin{tabular}{lllll}
\hline Gaskill 2009 & - & - & - & - \\
\hline Germain 2006 & - & - & - & - \\
\hline
\end{tabular}

\begin{tabular}{lll}
\hline Hankey 1993 & - \\
\hline Hickson 2004 & Grip strength & -
\end{tabular}

Length of stay (d), volume of fluids given

\section{Holyday 2012}

Length of stay, readmis- Estimated
sions

\begin{tabular}{ll}
\hline Johansen 2004 & Length of stay (LOS 28 ) \\
& $=$ LOS from admission \\
& to inclusion + LOS from \\
inclusion to discharge & (maximum 28 days) \\
& LOS $_{\mathrm{NDI}}=$ LOS 28 - num- \\
ber of final days with NDI & $=3$ ) \\
& NDI = index of mobility, \\
infections and complica- & tions
\end{tabular}

\section{Kraft 2012}

\begin{tabular}{lllll}
\hline Kretser 2003 & iADL, ADL, dependence & - & - & - \\
\hline Larsson 1990 & $\begin{array}{l}\text { Encompassed in the } \\
\text { Modified Norton Scale }\end{array}$ & - & - & - \\
\hline
\end{tabular}


(Continued)

\begin{tabular}{|c|c|c|c|c|c|}
\hline Lin 2010 & $\begin{array}{l}\text { Eating function (need for } \\
\text { verbal and/or physical } \\
\text { assistance or feeding + } \\
\text { eating time) }\end{array}$ & - & - & - & - \\
\hline Lin 2011 & $\begin{array}{l}\text { Eating function (need for } \\
\text { verbal and/or physical } \\
\text { assistance or feeding + } \\
\text { eating time) }\end{array}$ & - & - & - & - \\
\hline Mathey 2001a & - & $\begin{array}{l}\text { Biochemical } \\
\text { data }\end{array}$ & - & - & - \\
\hline Mathey 2001b & $\begin{array}{l}\text { Hunger, appetite and } \\
\text { sensory perception }\end{array}$ & - & - & - & - \\
\hline Munk 2014 & Handgrip strength & & Length of hospital stay & - & - \\
\hline Nijs 2006 & $\begin{array}{l}\text { Motor function (nursing } \\
\text { home physical perfor- } \\
\text { mance test) }\end{array}$ & - & - & - & - \\
\hline Olofsson 2007 & - & - & Length of hospital stay & - & - \\
\hline Pivi 2011 & - & $\begin{array}{l}\text { Biochemical } \\
\text { data }\end{array}$ & - & - & - \\
\hline Potter 2001 & $\begin{array}{l}\text { Functional recovery (20- } \\
\text { point Barthel ADL index) }\end{array}$ & - & $\begin{array}{l}\text { Length of hospital stay, } \\
\text { discharge placement }\end{array}$ & - & - \\
\hline Salva 2011 & ADL, iADL scores & $\begin{array}{l}\text { MMSE, CDR, } \\
\text { NPIQ }\end{array}$ & - & - & RUD score \\
\hline Silver 2008 & - & - & - & - & - \\
\hline Simmons 2008 & - & - & - & - & - \\
\hline Simmons 2010 & - & - & - & - & $\begin{array}{l}\text { Cost-effective- } \\
\text { ness }\end{array}$ \\
\hline Smolliner 2008 & $\begin{array}{l}\text { Handgrip strength, peak } \\
\text { flow, Barthel score, SF-36 } \\
\text { (physical function only) }\end{array}$ & - & - & - & - \\
\hline Splett 2003 & - & - & Hospital admissions & - & - \\
\hline Taylor 2006 & - & - & - & - & - \\
\hline Van den Berg 2015 & & & Length of stay & $\begin{array}{l}\text { Stated as } \\
\text { none but not } \\
\text { defined }\end{array}$ & - \\
\hline Van Ort & $\begin{array}{l}\text { Functional ability of par- } \\
\text { ticipant }\end{array}$ & - & - & - & - \\
\hline
\end{tabular}


(Continued)

ADL: activities of daily living; AMC: arm muscle circumference; BMI: body mass index; CDC: Centre for Disease Control; CDR: clinical dementia rating scale; $\mathrm{CHO}$ : carbohydrate; d: day; EQ-5D/EuroQol: European Quality of Life Scale; HGS: handgrip strength; iADL: instrumental Activities of Daily Living; MAC: mid-arm circumference; MAMC: mid-arm muscle circumference; MJ: mega joules; MMSE: Mini Mental State Examination; MNA: Mini Nutritional Assessment; NPIQ: Neuropyschiatric Inventory Question; PGCMS: Philadelphia Geriatric Centre Morale Scale; QOL: quality of life; RUD: reduction in use of health and social care scale; SF-36: Short Form - 36; SGA: subjective global assessment; SIP: sickness impact profile; TSF: triceps skinfold thickness

\section{Appendix 10. Adverse events}

\begin{tabular}{|c|c|c|c|c|c|}
\hline & Intervention(s) and comparator(s) & $\begin{array}{l}\text { Deaths } \\
(\mathrm{N} / \mathrm{N}(\%))\end{array}$ & $\begin{array}{l}\text { Participants } \\
\text { with } \\
\text { adverse } \\
\text { events } \\
(\mathrm{N} / \mathrm{N}(\%))\end{array}$ & $\begin{array}{l}\text { Participants } \\
\text { with } \\
\text { severe/seri- } \\
\text { ous adverse } \\
\text { events } \\
(\mathrm{N} / \mathrm{N}(\%))\end{array}$ & $\begin{array}{l}\text { Participants } \\
\text { discontinuing } \\
\text { trial } \\
\text { due to ad- } \\
\text { verse event } \\
(\mathrm{N} / \mathrm{N}(\%))\end{array}$ \\
\hline \multirow[t]{3}{*}{ Barton 2000} & I1: reduced portion fortified menu & - & - & - & - \\
\hline & I2: normal menu plus cooked breakfast & - & - & - & \\
\hline & C: normal hospital menu & - & - & - & - \\
\hline \multirow[t]{3}{*}{ Beck 2002} & I1: homemade oral supplement $(\mathrm{A})$ & - & - & - & - \\
\hline & I2: homemade oral supplement (B) & & & & \\
\hline & C: usual diet & - & - & - & - \\
\hline \multirow{2}{*}{$\begin{array}{l}\text { Bouillanne } \\
2013\end{array}$} & I: pulse diet (78\% protein at lunch) & $1 / 30(3.3)$ & - & - & - \\
\hline & $\begin{array}{l}\text { C: usual diet (protein distributed between } \\
\text { meals) }\end{array}$ & $1 / 36(2.8)$ & - & - & - \\
\hline \multirow{2}{*}{$\begin{array}{l}\text { Bourdel-Mar- } \\
\text { chasson } 2000\end{array}$} & I: oral supplementation + standard diet & $25 / 295(8.5)$ & - & - & - \\
\hline & C: standard diet & $22 / 377(5.8)$ & - & - & - \\
\hline \multirow{2}{*}{$\begin{array}{l}\text { Brouillette } \\
1991\end{array}$} & I: osmotherapy + activities & $1 / 10(10)$ & - & - & - \\
\hline & C: activities only & $0 / 10(0)$ & - & - & - \\
\hline \multirow{3}{*}{$\begin{array}{l}\text { Castellanos } \\
2009\end{array}$} & I1: fortified breakfast and lunch menu & - & - & - & - \\
\hline & 12: fortified lunch menu & - & - & - & - \\
\hline & C: usual menu & - & - & & - \\
\hline \multirow[t]{2}{*}{ Chang 2005} & I: training in feeding skills & - & - & - & - \\
\hline & C: no training & - & - & - & - \\
\hline Dennis 2005 & $\begin{array}{l}\text { I: nutritional supplement + normal hospital } \\
\text { diet }\end{array}$ & $241 / 2016(12)$ & $138 / 4023(3.4)$ & - & - \\
\hline
\end{tabular}


(Continued)

C: normal hospital diet 253/2007

(12.6)

\begin{tabular}{|c|c|c|c|c|c|}
\hline \multirow[t]{2}{*}{ Duncan 2006} & I. dietetic assistant & $19 / 145(13.1)$ & - & - & - \\
\hline & C: usual care & $36 / 157(22.9)$ & - & - & - \\
\hline \multirow[t]{4}{*}{ Essed 2007} & I1: monosodium glutamate & - & - & - & - \\
\hline & 12: flavour & - & - & - & - \\
\hline & 13: monosodium glutamate + flavour & - & - & - & - \\
\hline & C: maltodextrin & - & - & - & - \\
\hline \multirow[t]{2}{*}{ Essed 2009} & I: monosodium glutamate $+\mathrm{NaCl}$ & - & - & - & - \\
\hline & C: usual hot meal & - & - & - & - \\
\hline \multirow{2}{*}{$\begin{array}{l}\text { Faxen-Irving } \\
2011\end{array}$} & I: $3 \times 30 \mathrm{~mL}$ of fat emulsion daily & - & $5 / 34(14.7)$ & - & $5 / 34(14.7)$ \\
\hline & C: usual care & $2 / 37(5.4)$ & - & - & - \\
\hline \multirow[t]{2}{*}{ Gaskill 2009} & I: nutrition education programme & - & - & - & - \\
\hline & C: usual care & - & - & - & - \\
\hline \multirow[t]{2}{*}{ Germain 2006} & I: re-formed foods & - & - & - & - \\
\hline & C: usual diet & - & - & - & - \\
\hline \multirow[t]{2}{*}{ Hankey 1993} & I: oral nutritional supplement & - & $3 / 10(30)$ & - & - \\
\hline & C: standard hospital diet & - & $3 / 10(30)$ & - & - \\
\hline \multirow[t]{2}{*}{ Hickson 2004} & I: feeding assistance & $31 / 292(10.6)$ & - & - & \\
\hline & C: usual care & $35 / 300(11.7)$ & - & - & - \\
\hline \multirow[t]{2}{*}{ Holyday 2012} & I: malnutrition care plan & $1 / 72(1.4)$ & - & - & - \\
\hline & C: usual care & $4 / 72(5.6)$ & - & - & - \\
\hline \multirow{2}{*}{$\begin{array}{l}\text { Johansen } \\
2004\end{array}$} & I: nutrition team & - & - & - & - \\
\hline & C: usual care & - & - & - & - \\
\hline \multirow[t]{2}{*}{ Kraft 2012} & $\begin{array}{l}\text { I: oral nutritional supplement }+ \text { telemedicine } \\
\text { monitoring }\end{array}$ & - & - & - & $2 / 13(15.4)$ \\
\hline & C: usual care & - & - & - & - \\
\hline \multirow[t]{2}{*}{ Kretser 2003} & I: modified meals on wheels & $3 / 102(2.9)$ & - & - & - \\
\hline & C: traditional meals on wheels & $9 / 101(8.9)$ & - & - & - \\
\hline
\end{tabular}


(Continued)

\begin{tabular}{|c|c|c|c|c|c|}
\hline \multirow[t]{2}{*}{ Larsson 1990} & $\begin{array}{l}\text { I: oral nutritional supplement plus normal } \\
\text { hospital diet }\end{array}$ & 29/197 (14.7) & - & - & - \\
\hline & C: normal hospital diet & $56 / 238(23.5)$ & - & - & - \\
\hline Leslie 2012 & I: energy enriched meals & $2 / 19(10.5)$ & - & - & \\
\hline \multirow[t]{3}{*}{ Lin 2010} & I1: spaced-retrieval & - & - & - & - \\
\hline & 12: Montessori & - & - & - & - \\
\hline & C: usual care & - & - & - & - \\
\hline Lin 2011 & C: usual care & - & - & - & - \\
\hline \multirow{2}{*}{$\begin{array}{l}\text { Mathey } \\
2001 a\end{array}$} & I: improved meal ambiance & $7 / 21(33.3)$ & - & - & - \\
\hline & C: usual care & $5 / 17(29.4)$ & - & - & - \\
\hline \multirow{2}{*}{$\begin{array}{l}\text { Mathey } \\
\text { 2001b }\end{array}$} & I: flavour enhancement & - & - & - & - \\
\hline & C: usual care & - & - & - & - \\
\hline
\end{tabular}

\begin{tabular}{|c|c|c|c|c|c|}
\hline \multirow[t]{2}{*}{ Munk 2014} & $\begin{array}{l}\text { I: energy and protein enriched foods provid- } \\
\text { ed via a la carte menu in addition to hospital } \\
\text { food }\end{array}$ & $1 / 44(2.2)$ & \multicolumn{3}{|l|}{-} \\
\hline & C: usual care & $1 / 40(2.5)$ & - & & \\
\hline \multirow[t]{2}{*}{ Nijs 2006} & I: family-style meals & $18 / 112(16.1)$ & & - & - \\
\hline & C: usual care & $16 / 133(12.0)$ & - & - & - \\
\hline \multirow[t]{2}{*}{$\begin{array}{l}\text { Olofsson } \\
2007\end{array}$} & $\begin{array}{l}\text { I: multi-component intervention (including } \\
\text { nutrition) }\end{array}$ & $9 / 102(8.8)$ & - & - & - \\
\hline & C: usual care & $13 / 97(13.4)$ & - & - & - \\
\hline \multirow[t]{3}{*}{ Pivi 2011} & I1: nutrition education & - & - & - & - \\
\hline & 12: oral nutritional supplements & - & - & - & - \\
\hline & C: usual care & - & - & - & - \\
\hline \multirow[t]{2}{*}{ Potter 2001} & $\begin{array}{l}\text { I: oral nutritional supplement + normal hos- } \\
\text { pital diet }\end{array}$ & $21 / 186(11.3)$ & \multirow{2}{*}{$\begin{array}{l}\text { Reported "no } \\
\text { serious ad- } \\
\text { verse events" }\end{array}$} & - & - \\
\hline & C: normal hospital diet & $33 / 195(16.9)$ & & - & - \\
\hline \multirow{2}{*}{$\begin{array}{l}\text { Remsburg } \\
2001\end{array}$} & I: buffet-style meals & - & - & - & - \\
\hline & C: usual care & - & - & - & - \\
\hline
\end{tabular}


(Continued)

\begin{tabular}{|c|c|c|c|c|c|}
\hline \multirow[t]{2}{*}{ Salva 2011} & I: teaching and training & $43 / 448(9.6)$ & - & - & - \\
\hline & C: usual care & $29 / 498(5.8)$ & - & - & - \\
\hline \multirow[t]{2}{*}{ Silver 2008} & I: fortified home-delivered lunch & - & - & - & - \\
\hline & C: usual home-delivered lunch & - & & - & - \\
\hline \multirow{2}{*}{$\begin{array}{l}\text { Simmons } \\
2008\end{array}$} & I: feeding assistance and/or snacks & - & - & - & - \\
\hline & C: usual diet & - & - & - & - \\
\hline \multirow{2}{*}{$\begin{array}{l}\text { Simmons } \\
2010\end{array}$} & I1: snacks & - & - & - & - \\
\hline & C: usual care & - & - & - & - \\
\hline \multirow{2}{*}{$\begin{array}{l}\text { Smolliner } \\
2008\end{array}$} & I: fortified meals and snacks & $2 / 31(6.5)$ & - & - & - \\
\hline & C: usual diet & $1 / 34(2.9)$ & - & - & - \\
\hline \multirow[t]{2}{*}{ Splett 2003} & I: medical nutrition therapy & - & - & - & - \\
\hline & C: usual care & - & - & - & - \\
\hline \multirow[t]{2}{*}{ Taylor 2006} & I: 5-meal menu & - & - & - & - \\
\hline & C: usual (3-meal menu) & - & - & - & - \\
\hline \multirow{2}{*}{$\begin{array}{l}\text { V an den Berg } \\
2015\end{array}$} & $\begin{array}{l}\text { I2: } 62 \mathrm{~mL} \text { ONS four times daily with medica- } \\
\text { tion round }\end{array}$ & $2 / 80(2.5)$ & & & $\begin{array}{l}\text { 9/66 (13.6) (re- } \\
\text { fused further } \\
\text { ONS) }\end{array}$ \\
\hline & $\begin{array}{l}\text { C: usual care ( } 125 \mathrm{~mL} \text { ONS offered in be- } \\
\text { tween meals) }\end{array}$ & $4 / 88(4.5)$ & & & $\begin{array}{l}11 / 80(13.8) \\
\text { (refused fur- } \\
\text { ther ONS) }\end{array}$ \\
\hline \multirow[t]{2}{*}{ Van Ort 1995} & I: contextual and behavioural intervention & - & - & - & - \\
\hline & C: usual care & - & - & - & - \\
\hline
\end{tabular}

\section{Appendix 11. Survey of authors' providing information on trials}

\begin{tabular}{llll}
\hline $\begin{array}{l}\text { Trial author } \\
\text { contacted }\end{array}$ & $\begin{array}{l}\text { Trial author } \\
\text { replied }\end{array}$ & $\begin{array}{l}\text { Trial author } \\
\text { provided data }\end{array}$ & Comments \\
\hline
\end{tabular}


(Continued)

\begin{tabular}{|c|c|c|c|c|}
\hline Barton 2000 & Yes & Yes & Yes & Additional data not used \\
\hline Beck 2002 & Yes & Yes & Yes & Additional data not used \\
\hline $\begin{array}{l}\text { Bourdel-Marchasson } \\
2000\end{array}$ & Yes & Yes & Yes & $\begin{array}{l}\text { Not used, and unable to provide data re- } \\
\text { quested on weight }\end{array}$ \\
\hline Bouillanne 2013 & Yes & Yes & Yes & Data received on weight and energy intake \\
\hline Brouillette 1991 & No & $\mathrm{N} / \mathrm{A}$ & $\mathrm{N} / \mathrm{A}$ & \\
\hline Castellanos 2009 & Yes & No & $\mathrm{N} / \mathrm{A}$ & \\
\hline Chang 2005 & Yes & No & $\mathrm{N} / \mathrm{A}$ & \\
\hline Dennis 2005 & Yes & Yes & Yes & Information used on complication rates \\
\hline Duncan 2006 & Yes & Yes & Yes & Awaiting data on length of stay \\
\hline Essed 2007 & Yes & No & $\mathrm{N} / \mathrm{A}$ & \\
\hline Essed 2009 & Yes & No & $\mathrm{N} / \mathrm{A}$ & \\
\hline Faxen-Irving 2011 & Yes & Yes & Yes & $\begin{array}{l}\text { Data on energy intake, length of stay, BMI } \\
\text { and ADLs provided. No data available on in- } \\
\text { fections }\end{array}$ \\
\hline Gaskill 2009 & Yes & Yes & No & Assume unable to provide data \\
\hline Germain 2006 & Yes & Yes & Yes & $\begin{array}{l}\text { Data provided for BMI mean and SD of } \\
\text { change }\end{array}$ \\
\hline Hankey 1993 & Yes & No & $\mathrm{N} / \mathrm{A}$ & \\
\hline Hickson 2004 & Yes & Yes & Yes & $\begin{array}{l}\text { Author unable to provide this data on ener- } \\
\text { gy intake and hospital readmission as it was } \\
\text { not measured, therefore not usable. Data } \\
\text { provided on complications as requested }\end{array}$ \\
\hline Holyday 2012 & Yes & Yes & Yes & $\begin{array}{l}\text { Data obtained and used for hospital read- } \\
\text { mission rates }\end{array}$ \\
\hline Johansen 2004 & Yes & No & $\mathrm{N} / \mathrm{A}$ & Data not used \\
\hline Kraft 2012 & Yes & No & $\mathrm{N} / \mathrm{A}$ & \\
\hline Kretser 2003 & No & $\mathrm{N} / \mathrm{A}$ & $\mathrm{N} / \mathrm{A}$ & Unable to find contact for author \\
\hline Larsson 1990 & Yes & No & $\mathrm{N} / \mathrm{A}$ & Data not used \\
\hline Lin 2010 & Yes & No & $\mathrm{N} / \mathrm{A}$ & \\
\hline Lin 2011 & No & $\mathrm{N} / \mathrm{A}$ & $\mathrm{N} / \mathrm{A}$ & \\
\hline Mathey 2001a & Yes & No & $\mathrm{N} / \mathrm{A}$ & \\
\hline
\end{tabular}


(Continued)

\begin{tabular}{|c|c|c|c|c|}
\hline Mathey 2001b & Yes & No & $\mathrm{N} / \mathrm{A}$ & \\
\hline Nijs 2006 & No & $\mathrm{N} / \mathrm{A}$ & $\mathrm{N} / \mathrm{A}$ & \\
\hline Olofsson 2007 & Yes & Yes & Yes & Data used for BMI, weight and complications \\
\hline Pivi 2011 & Yes & No & $\mathrm{N} / \mathrm{A}$ & \\
\hline Potter 2001 & Yes & No & $\mathrm{N} / \mathrm{A}$ & \\
\hline Remsburg 2001 & No & $N / A$ & $\mathrm{~N} / \mathrm{A}$ & \\
\hline Salva 2011 & Yes & No & $\mathrm{N} / \mathrm{A}$ & \\
\hline Silver 2008 & No & $\mathrm{N} / \mathrm{A}$ & $\mathrm{N} / \mathrm{A}$ & \\
\hline Simmons 2008 & Yes & Yes & No & Data not available \\
\hline Simmons 2010 & Yes & Yes & No & Data not available \\
\hline Smolliner 2008 & Yes & Yes & Yes & $\begin{array}{l}\text { Data provided for mean and SD of change } \\
\text { for weight, BMI, handgrip, and QoL }\end{array}$ \\
\hline Splett 2003 & Yes & No & $\mathrm{N} / \mathrm{A}$ & \\
\hline Taylor 2006 & No & $\mathrm{N} / \mathrm{A}$ & $\mathrm{N} / \mathrm{A}$ & \\
\hline Van Ort 1995 & Yes & No & $\mathrm{N} / \mathrm{A}$ & \\
\hline Leslie 2012 & No & $\mathrm{N} / \mathrm{A}$ & $\mathrm{N} / \mathrm{A}$ & \\
\hline Munk 2014 & No & $\mathrm{N} / \mathrm{A}$ & $\mathrm{N} / \mathrm{A}$ & \\
\hline V an den Berg 2015 & Yes & Yes & Yes & $\begin{array}{l}\text { The clinical trial register number did not al- } \\
\text { low the trial to be identified within the reg- } \\
\text { ister. The authors provided a link to the trial } \\
\text { protocol via the WHO International Clinical } \\
\text { Trials Registry Platform. }\end{array}$ \\
\hline
\end{tabular}

ADL: activities of daily living; BMI: body mass index; N/A: not applicable; QoL: (health-related) quality of life; SD: standard deviation: WHO World Health Organisation 


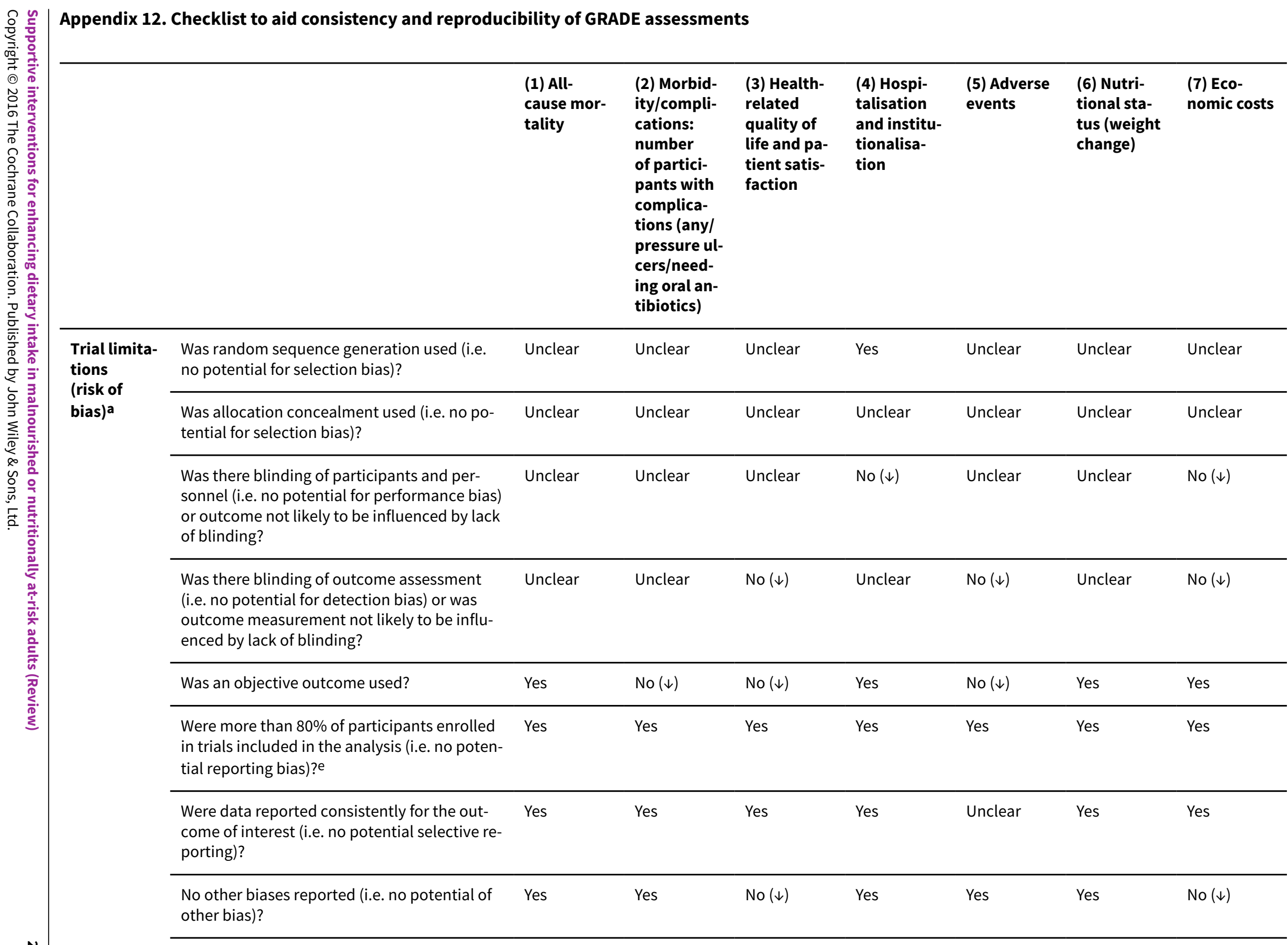


Did the trials end up as scheduled (i.e. not

Yes

Yes

Yes

Yes

Yes

Yes

Yes stopped early)?

\begin{tabular}{|c|c|}
\hline \multirow{2}{*}{$\begin{array}{l}\text { Inconsis- } \\
\text { tencyb }\end{array}$} & Point estimates did not vary widely? \\
\hline & $\begin{array}{l}\text { To what extent did confidence intervals over- } \\
\text { lap (substantial: all confidence intervals over- } \\
\text { lap at least one of the included studies point } \\
\text { estimate; some: confidence intervals over- } \\
\text { lapped but not all overlapped at least } 1 \text { point } \\
\text { estimate; no: at least } 1 \text { outlier: where the con- } \\
\text { fidence interval of some of the studies did not } \\
\text { overlap with those of most included studies)? }\end{array}$ \\
\hline
\end{tabular}

overlap with those of most included studies)?

Wes

What was the magnitude of statistical hetero- Low

geneity (as measured by $\left.I^{2}\right)$ - low $\left(I^{2}<40 \%\right)$,

moderate $\left(I^{2} 40 \%-60 \%\right)$, high $\left.I^{2}>60 \%\right)$ ?

\begin{tabular}{lllllll} 
Yes & No $(\downarrow)$ & N/A & Yes & N/A & Yes & N/A \\
\hline Substantial & Some & N/A & Substantial & N/A & Substantial & N/A \\
& & & & & \\
& & & & & \\
& & & & \\
\hline
\end{tabular}

Was the test for heterogeneity statistically significant $(P<0.1)$ ?

Nots

Not statisti-
cally significant

$\begin{array}{lll}\text { No }(\downarrow) & \text { N/A } & \text { Yes } \\ \operatorname{High}(\downarrow) & \text { N/A } & \text { Mode }\end{array}$

Yes N/A

Moderate

N/A

Moderate

N/A

\begin{tabular}{llll} 
& cant & $(\downarrow)$ \\
\hline Indirect- $\quad$ Were the populations in included studies ap- & Highly ap- $\quad$ H
\end{tabular}

nessa

plicable to the decision context?
Were the interventions in the included studies

applicable to the decision context? plicable

Statistically N/A

significant

Not statisti-

N/A

Statistically

N/A

Was the included outcome not a surrogate Yes

outcome?

Was the outcome timeframe sufficient?

$\begin{array}{lllllll}\begin{array}{l}\text { Highly ap- } \\ \text { plicable }\end{array} & \begin{array}{l}\text { Highly ap- } \\ \text { plicable }\end{array} & \begin{array}{l}\text { Highly ap- } \\ \text { plicable }\end{array} & \begin{array}{l}\text { Highly ap- } \\ \text { plicable }\end{array} & \begin{array}{l}\text { Highly ap- } \\ \text { plicable }\end{array} & \begin{array}{l}\text { Highly ap- } \\ \text { plicable }\end{array} & \begin{array}{l}\text { Highly ap } \\ \text { plicable }\end{array}\end{array}$

Highly ap-

cant

significant

Highly ap-

Highly ap- Highly

Highly ap-

(

Were the conclusions based on direct com

parisons?

Impreci- Was the confidence interval for the pooled es- Yes

sionc timate not consistent with benefit and harm?

What is the magnitude of the median sample size (high: 300 participants, intermedi-

Intermedi-

ate to high

ate

\begin{tabular}{|c|c|c|c|c|c|}
\hline $\begin{array}{l}\text { hly ap- } \\
\text { cable }\end{array}$ & $\begin{array}{l}\text { Highly ap- } \\
\text { plicable }\end{array}$ & $\begin{array}{l}\text { Highly ap- } \\
\text { plicable }\end{array}$ & $\begin{array}{l}\text { Highly ap- } \\
\text { plicable }\end{array}$ & $\begin{array}{l}\text { Highly ap- } \\
\text { plicable }\end{array}$ & $\begin{array}{l}\text { Highly ap- } \\
\text { plicable }\end{array}$ \\
\hline
\end{tabular}

Highly ap- Highly ap-

Highly ap-

Highly ap- Highly ap- Highly ap- Highly ap- Highly ap-

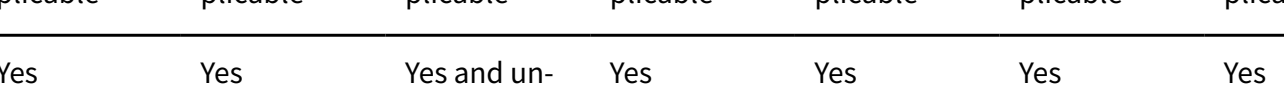

Yes

Yros

Sufficient

Sufficient

Sufficient

Yus

Yes

Yes N/A

No $(\downarrow)$

N/A

ufficient

Sufficient

\begin{tabular}{ll} 
Sufficient & Sufient \\
\hline Yes
\end{tabular}

Yes Yes Yes

Yes

N/A




\begin{tabular}{|c|c|c|c|c|c|c|c|c|}
\hline \multirow[t]{3}{*}{ (Continued) } & \multicolumn{8}{|l|}{$\begin{array}{l}\text { ate: } 100-300 \text { participants, low: }<100 \text { partici- } \\
\text { pants)?e }\end{array}$} \\
\hline & $\begin{array}{l}\text { What was the magnitude of the number of in- } \\
\text { cluded studies (large: }>10 \text { studies, moderate: } \\
5-10 \text { studies, small: }<5 \text { studies)?e }\end{array}$ & Large & Moderate & Moderate & Moderate & Small $(\downarrow)$ & Large & Small $(\downarrow)$ \\
\hline & $\begin{array}{l}\text { Was the outcome a common event (e.g. oc- } \\
\text { curs more than } 1 / 100) \text { ? }\end{array}$ & Yes & Yes & $\mathrm{N} / \mathrm{A}$ & $\mathrm{N} / \mathrm{A}$ & Yes & $\mathrm{N} / \mathrm{A}$ & $\mathrm{N} / \mathrm{A}$ \\
\hline \multirow{6}{*}{$\begin{array}{l}\text { Publication } \\
\text { biasd }\end{array}$} & Was a comprehensive search conducted? & Yes & Yes & Yes & Yes & Yes & Yes & Yes \\
\hline & Was grey literature searched? & Yes & Yes & Yes & Yes & Yes & Yes & Yes \\
\hline & $\begin{array}{l}\text { Were no restrictions applied to study selec- } \\
\text { tion on the basis of language? }\end{array}$ & Yes & Yes & Yes & Yes & Yes & Yes & Yes \\
\hline & $\begin{array}{l}\text { There was no industry influence on studies in- } \\
\text { cluded in the review? }\end{array}$ & Unclear & Unclear & Unclear & Unclear & Unclear & Unclear & Unclear \\
\hline & $\begin{array}{l}\text { There was no evidence of funnel plot asym- } \\
\text { metry? }\end{array}$ & Unclear & Unclear & $\mathrm{N} / \mathrm{A}$ & Unclear & Unclear & Unclear & $\mathrm{N} / \mathrm{A}$ \\
\hline & $\begin{array}{l}\text { There was no discrepancy in findings be- } \\
\text { tween published and unpublished trials? }\end{array}$ & Unclear & Unclear & Unclear & Unclear & Unclear & Unclear & Unclear \\
\hline \multicolumn{9}{|c|}{$\begin{array}{l}\text { aQuestions on risk of bias are answered in relation to the majority of the aggregated evidence in the meta-analysis rather than to individual trials } \\
\text { bQuestions on inconsistency are primarily based on visual assessment of forest plots and the statistical quantification of heterogeneity based on } I^{2} \\
\text { cWhen judging the width of the confidence interval it is recommended to use a clinical decision threshold to assess whether the imprecision is clinically meaningful } \\
\text { dQuestions address comprehensiveness of the search strategy, industry influence, funnel plot asymmetry and discrepancies between published and unpublished trials } \\
\text { eDepends on the context of the systematic review area } \\
\text { ( } \downarrow \text { ): key item for possible downgrading the quality of the evidence (GRADE) as shown in the footnotes of the 'Summary of finding' table(s); GRADE: Grading of Recommenda- } \\
\text { tions Assessment, Development and Evaluation; N/A: not applicable }\end{array}$} \\
\hline
\end{tabular}




\section{CONTRIBUTIONSOFAUTHORS}

All authors have read, commented and contributed to the preparation of review manuscripts.

Michelle Gibbs (MG): protocol draft, search strategy development, acquisition of copies of trials, trial selection, data extraction, and future review updates.

Katherine Kimber (KK): trial selection, data extraction, data analyses, data interpretation, and future review updates.

Christine Baldwin (CB): protocol draft, trial selection, data extraction, data analysis, data interpretation and completed revision of the review following peer review, and future review updates.

Christine Elizabeth Weekes (CEW): protocol draft, trial selection, data extraction, data analysis, data interpretation and completed revision of the review following peer review, and future review updates.

\section{DECLARATIONS OF INTEREST}

Michelle Gibbs: this work was financially supported by a grant from the British Dietetic Association.

Katherine Kimber: none known.

Christine Baldwin: some of the early work on this review was funded by an educational grant from the British Dietetic Association. The grant was used to support the salary of two research assistants who contributed to the searching, study selection and writing of the review.

Christine Elizabeth Weekes: none known.

\section{SOURCES OF SUPPORT}

\section{Internal sources}

- No sources of support supplied

\section{External sources}

- British Dietetic Association, UK.

This review was part funded by a grant from the British Dietetic Association.

\section{DIFFERENCES BETWEEN PROTOCOLANDREVIEW}

Katherine Kimber began work on this review after publication of the protocol. At the protocol stage it was anticipated that searching of Greynet would be undertaken but this was not done and so the sections on electronic searching and searching other resources have been amended.

Since the publication of the protocol of this review and the final review draft a considerable time has elapsed which demanded a number of changes to the protocol such as specification of a number of additional secondary outcomes (which are mandatory within the CMED Group), specification of outcomes for the 'Summary of findings' table and specification of timing of outcome measurement. Also the updated search strategy was focused on major databases and differed slightly from the older versions mainly due to changes in the database structure over time.

We could not investigate a number of prespecified subgroup and sensitivity analyses because of lack of data. Also, cross-over trials did not contribute to the effect estimates established by meta-analyses because data were not available from baseline to the end of phase 1 of the cross-over trials to be included in meta-analyses.

\section{N O T E S}

Portions of the methods sections, the appendices, additional tables and figures 1 to 3 of this review are based on a standard template established by Cochrane Metabolic and Endocrine Disorders.

\section{INDEX TERMS}

\section{Medical Subject Headings (MeSH)}

*Dietary Supplements [adverse effects]; *Meals; Cause of Death; Dietary Proteins [administration \& dosage]; Energy Intake; Environment; Hospitalization [statistics \& numerical data]; Malnutrition [*diet therapy] [mortality]; Nutritional Status; Quality of Life; Randomized Controlled Trials as Topic 


\section{MeSH check words}

Adult; Humans 